DOCUMENT OCCASIONNEL

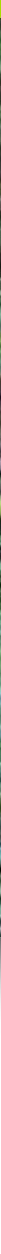

\title{
Guide pour mieux connaître les impacts des projets de REDD+ sur la subsistance des populations
}

Pamela Jagger

Erin O. Sills

Kathleen Lawlor

William D. Sunderlin

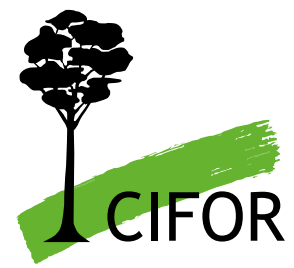





\title{
Guide pour mieux connaître les impacts des projets de REDD+ sur la subsistance des populations
}

\author{
Pamela Jagger \\ Université de Caroline du Nord à Chapel Hill \\ CIFOR \\ Erin O. Sills \\ Université de I'État de Caroline du Nord \\ CIFOR \\ Kathleen Lawlor \\ Université de Caroline du Nord à Chapel Hill \\ William D. Sunderlin \\ CIFOR
}


Document Occasionnel 73

(C2011 Center for International Forestry Research Tous droits réservés

ISBN 978-602-8693-70-7

Jagger P., Sills, E.O., Lawlor, K. et Sunderlin, W.D. 2011 Guide pour mieux connaître les impacts des projets de REDD+ sur la subsistance des populations. Document occasionnel 73, CIFOR, Bogor, Indonésie.

Traduction de : Jagger P., Sills, E.O., Lawlor, K. and Sunderlin, W.D. 2010 A guide to learning about livelihood impacts of REDD+ projects. Occasional paper 56. CIFOR, Bogor, Indonesia.

Photographie de couverture : Deux habitants des forêts rentrent chez eux après le ramassage du bois de feu dans le district de Ketapang, à Kalimantan Ouest, Indonésie (๔ Andini Desita Ekaputri/CIFOR)

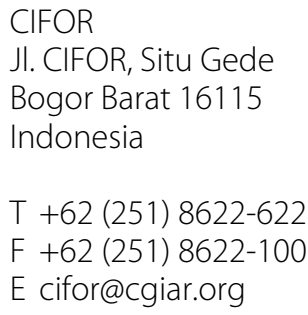

\section{www.cifor.org}

Les opinions exprimées dans cette publication sont celles des auteurs. À ce titre, elles ne reflètent pas nécessairement le point de vue du CIFOR, ni celui des institutions auxquelles appartiennent les auteurs, ni celui des commanditaires de cette publication. 


\section{Table des matières}

Liste des figures, tableaux et encadrés iv

$\begin{array}{ll}\text { Liste des abréviations } & \mathbf{v}\end{array}$

Remerciements vii

Résumé $\quad$ ix

Préface $\quad$ xiv

1 Tirer les enseignements de la REDD+ : un exercice nécessaire 1

1.1 Pourquoi est-il nécessaire de tirer les enseignements des projets de REDD+ ? 1

1.2 Pourquoi faut-il évaluer l'impact des projets sur le bien-être économique et social ? 5

$\begin{array}{ll}1.3 & \text { Quels projets faut-il évaluer? }\end{array}$

1.4 Tirer les enseignements du passé $\quad 9$

$\begin{array}{ll}1.5 \text { Comment s'y prendre } & 10\end{array}$

2 Utiliser le raisonnement contrefactuel pour mieux connaître les projets de REDD+ 11

2.1 Choix de la méthodologie de recherche : hypothèses fondamentales 13

2.2 Choix d'une méthodologie de recherche : questions fondamentales 15

2.3 Choix d'une méthodologie de recherche : options fondamentales 16

2.4 Mise en œuvre de la méthodologie de recherche 23

3 Comprendre les phénomènes de causalité reliant les interventions de REDD+ $\begin{array}{ll}\text { aux résultats } & \mathbf{2 7}\end{array}$

3.1 Comprendre « l'objet » et « les raisons » de l'évaluation d'impact 27

3.2 Situer l'élaboration du modèle de causalité dans la conception de l'évaluation
d'impact

3.3 Représenter et mettre à l'épreuve les modèles de causalité 30

4 Aspects pratiques à prendre en compte pour mieux comprendre les impacts de la REDD+ sur le bien-être économique et social 39

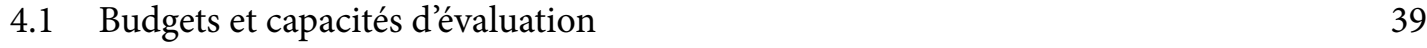

$\begin{array}{lll}4.2 & \text { Aspects éthiques } & 40\end{array}$

5 Faire avancer la réalisation de la REDD+ : principes pour mieux connaître les impacts sociaux $\quad 43$

$\begin{array}{ll}\text { Bibliographie } & 46\end{array}$

$\begin{array}{ll}\text { Glossaire } & 57\end{array}$

Annexes

A Fiches techniques $\quad 61$

$\begin{array}{lr}\text { B Bibliographie analytique } & 97\end{array}$

C À propos des recommandations techniques et des instruments d'enquête 111 


\section{Liste des figures, tableaux et encadrés}

\section{Flgures}

1 Répartition mondiale des projets de carbone forestier

2 Arbre décisionnel pour le choix d'une méthodologie de recherche

3 Attribution erronée d'une baisse du bien-être à un projet REDD+ suite à une comparaison avant-après

4 Attribution erronée d'une baisse du bien-être économique à un projet de REDD+ suite à une comparaison témoin-intervention sans appariement

\section{Tableaux}

1 Possibilités de méthodologies de recherche ayant pour objectif l'évaluation d'impact ex post fondée sur l'évidence empirique

2 Éléments pour la représentation de la chaîne causale

\section{Encadrés}

1 Répartition mondiale des projets de REDD+

2 Pourquoi il est possible de tirer les enseignements des projets de REDD+

3 Normes et systèmes de certification pour les projets de REDD+

4 Étude comparative mondiale du CIFOR sur la REDD+

5 Le problème du raisonnement contrefactuel contrefait

6 Comparaison des modèles de causalité reliant les interventions et leurs résultats

7 Étude de la mise en œuvre de projet dans le cadre de la GCS-REDD+

8 Hypothèses fondamentales posées par l'étude GCS-REDD+ 


\section{Liste des abréviations}

3E+

ACR

ASP

ATE

ATT

BACI

BAG

BNS

BR

CCBA

CCNUCC

CIFOR

CMED

COP 13

CPS

DCP

EIP

EP

EPMR

FIDA

FPIC

GCS

GCS-REDD+
Efficacité, efficience, équité et co-bénéfices

Registre américain du carbone

American Carbon Registry

Appariement par score de propension

Effet moyen du traitement

Average treatment effect

Effet moyen du traitement sur les traités

Average treatment effect on treated

Comparaison avant-après/contrôle-intervention

Before-after/control-intervention

Guide de l'évaluation de base

Basic Assessment Guide

Enquête sur les besoins fondamentaux

Basic Necessities Survey

Boisement/reboisement

Alliance Climat, Communauté et Biodiversité

Climate, Community and Biodiversity Alliance

Convention-cadre des Nations Unies sur les changements climatiques

Centre de recherche forestière internationale

Cadre des moyens d'existence durables

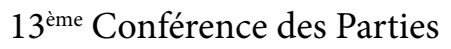

Changement le plus significatif

Document de conception de projet

Étude d'impact participative

Évaluation des porteurs de projet

Évaluation participative en milieu rural

Fonds international de développement agricole

Consentement libre, préalable et éclairé

Free, prior and informed consent

Global Conservation Standard

Étude comparative mondiale du CIFOR sur le programme REDD+

Global Comparative Study on REDD+

GIEC

Groupe d'experts intergouvernemental sur l'évolution du climat 
HLSA

IDH

IFRI

IMC

LOAM

LSMS

$M \& E$

MDP

MNV

NER

NONIE

OCDE

ONU

PEN

PNUD

PSE

REDD

REDD+

SAPA

SPI

SUTVA

VCS

VCU

WCS
Analyses de la sécurité des moyens d'existence des ménages

Household Livelihood Security Assessment

Indice de développement humain

Programme international de recherche sur les institutions et les ressources forestières International Forestry Resources and Institutions

Indice de masse corporelle

Méthode d'évaluation des situations paysagères

Landscape Outcomes Assessment Methodology

Étude de mesure du niveau de vie

Living Standards Measurement Study

Surveillance et évaluation

Monitoring and evaluation

Mécanisme pour un développement propre

Mesure, notification et vérification

Monitoring, reporting and verification

Niveau d'émission de référence

Initiative du Réseau des réseaux pour l'évaluation d'impact

Network of Networks Impact Evaluation Initiative

Organisation de coopération et de développement économiques

Organisation des Nations Unies

Réseau pauvreté et environnement

Poverty Environment Network

Programme des Nations Unies pour le développement

Paiements pour services environnementaux, paiements pour services écosystémiques

Réduction des émissions liées à la déforestation et à la dégradation des forêts

Réduction des émissions liées à la déforestation et à la dégradation des forêts et renforcement des stocks de carbone forestier

Évaluation sociale des aires protégées

Social Assessment of Protected Areas

Étude de la mise en œuvre de projet

Survey of project implementation

Hypothèse d'absence d'effets de diffusion du traitement

Stable unit treatment value assumption

Norme de compensation volontaire de carbone

Voluntary Carbon Standard

Unités de crédit carbone volontaires

Voluntary Carbon Units

Wildlife Conservation Society 


\section{Remerciements}

$\mathrm{N}$ ous sommes redevables à un grand nombre d'organisations et de personnes d'avoir apporté leur contribution au contenu et à la structure de ce guide, ainsi quà sa réalisation. Ces travaux ont été soutenus par l'Agence norvégienne de coopération pour le développement, l'Agence australienne d'aide au développement international, le ministère britannique du Développement international, la Commission européenne, le Département finlandais pour la coopération au développement international, la Fondation David et Lucile Packard, le Programme sur les forêts (PROFOR), l'Agence américaine pour le développement international, ainsi que le Service des forêts du Département de l'agriculture aux États-Unis. Nous remercions vivement RTI International de nous avoir accordé une subvention ayant permis malgré sa taille modeste, de soutenir un groupe de travail et un séminaire interuniversitaire consacré à la REDD+. L'Université de Caroline du Nord à Chapel Hill (UNC-CH) et l'Université de l'État de Caroline du Nord (NCSU) ont apporté un soutien institutionnel. Nous tenons notamment à remercier Megha Karmalkar de l'UNC-CH et Liwei Lin de la NCSU pour l'appui qu'ils ont apporté au projet en leur qualité d'assistants de recherche, le premier dans le cadre du premier cycle d'études universitaires et le second dans le cadre des cycles supérieurs. L'attachée de recherche postdoctorale à la NCSU, Katrina Mullen, a apporté une aide précieuse en ce qui concerne la qualité des données de rétrospection. Nos familles, et en particulier Adam Lowe et Subhrendu Pattanayak, nous ont fourni un soutien logistique et moral tout au long de la rédaction de ce guide.

Nous avons eu la chance de profiter des propositions avancées à l'occasion de différents forums, utiles tant pour le fond que le plan de ce guide. Nos réflexions initiales se sont structurées pendant la rédaction du chapitre 22 de l'ouvrage Réaliser la REDD+ (Jagger et al. 2009) coécrit avec Stibniati Atmadja et Subhrendu Pattanayak. Alors que nos idées prenaient forme, nous avons bénéficié des commentaires très utiles d'Arild Angelsen. Un atelier intitulé «l'Étude comparative mondiale du CIFOR sur la REDD+ : bilan des méthodes et des meilleures pratiques pour l'évaluation des projets de REDD+ » s'est tenu en Caroline du Nord en janvier 2010. Les discussions que nous avons eues avec les participants à cet atelier ont été très productives. Citons notamment Soeryo Adiwibowo, Andre Rodrigues Aquino, Stibniati Atmadja, Simone Bauch, Rizaldi Boer, Miguel Calmon, Susan Caplow, Mariano Cenamo, Paul Ferraro, Alain Karsenty, Anirudh Krishna, Liwei Lin, Erin Myers Madeira, Will Makin, Subhrendu Pattanayak, Mustofa Agung Sardjono, Frances Seymour, Satyawan Sunito, Peter Vaughan, Jeff Vincent et Sven Wunder. Nous avons également profité des commentaires extrêmement précieux suite à la première présentation de nos idées lors d'un atelier organisé par l'Alliance Climat, Communauté et Biodiversité en mai 2010 et intitulé «Atelier sur l'évaluation des impacts sociaux et environnementaux des projets liés au carbone dans le sol».

Ce guide s'est construit grâce aux nombreux apports collectés lors de conversations et d'échanges de courriels au sujet de la REDD+ et des évaluations d'impact, que nous avons eus avec de nombreux collègues, dont Arild Angelsen, Amy Duchelle, Joanna Durbin, Paul Ferraro, Cecilia Luttrell, Erin Myers Madeira, Steve Panfil, Subhrendu Pattanayak, Daju Resosudarmo, et Michael Richards. Les réactions du groupe de travail Triangle sur la REDD+, de Brian Murray et des étudiants ayant 
participé au séminaire interuniversitaire ont été très utiles pour la formulation de notre pensée. Nous avons par ailleurs bénéficié de la révision perspicace d'Andrew Wardell, de deux pairs universitaires anonymes, ainsi que de la lecture critique de deux praticiens anonymes. Leurs contributions ont énormément renforcé la qualité de ce guide. Nous tenons à remercier les nombreuses personnes qui ont apporté leur concours appréciable aux instruments de recherche de l'Étude comparative mondiale du CIFOR sur la REDD+ (GCS-REDD+), inclus dans ce document.
Enfin, la réalisation de ce travail a été soumise à de fortes contraintes de temps. Plusieurs collaborateurs du Groupe des services d'information du CIFOR ont travaillé sans relâche à l'élaboration de ce guide dont le lancement était prévu à la Conférence 2010 de la CCNUCC sur les changements climatiques de Cancún au Mexique. Nous sommes particulièrement reconnaissants à Beth Varley, Hélène Piantone, Imogen Badgery-Parker, Vidya Fitrian, Edith Johnson, Glen Mulcahy, Andri Novianto, Handi Priono et Gideon Suharyanto. 


\section{Résumé}

$\mathrm{D}$ ans ce guide, nous cherchons à comprendre les impacts des projets de REDD+ de première génération sur les conditions de vie des populations. Ces projets ont été programmés et financés par tout un ensemble d'acteurs, dans le but de mettre en œuvre des interventions très diverses visant à réduire la déforestation et la dégradation des forêts, agir en faveur de la conservation et de la gestion durable des forêts et renforcer les stocks de carbone forestier. C'est vers ces projets que se tourne la communauté internationale lorsqu'il s'agit de mieux comprendre et de s'orienter dans la conception de la REDD+. Il est évident que les moyens de mise en ouvre de la REDD+ au plan infranational sont limités, ainsi que les résultats quelle peut obtenir. Par conséquent, il ne faut pas s'attendre à ce que notre expérience des projets déjà mis en œuvre apporte les réponses à toutes nos questions relatives à la REDD+. Cependant, par l'utilisation de méthodologies de recherche rigoureuses et la représentation des chaînes de causalité des projets, nous pouvons recueillir des informations très utiles sur l'influence des interventions de REDD+ sur le bien-être économique et social dans les régions forestières. Ce guide fournit une synthèse de ces méthodologies.

Sa partie centrale est axée sur les composantes d'une méthodologie de recherche et d'un travail rigoureux de représentation causale. Nous défendons la thèse que le mieux pour tirer les enseignements de projets est de recourir à une approche méthodologique mixte d'évaluation d'impact des plus rigoureuses, avec pour objectif de quantifier et d'interpréter les impacts à la lumière d'une théorie du changement. Ce guide comprend une série de fiches techniques (Annexe A), ainsi qu'une bibliographie analytique comprenant des boîtes à outils, des méthodes et des résultats de recherche pertinents pour l'étude des impacts des projets de REDD+ sur le bien-être économique et social (Annexe B).

Le Centre de recherche forestière internationale (CIFOR) met en place une base de données scientifiques sur le programme REDD+ via l'Étude comparative mondiale du CIFOR sur la REDD+ (GCS-REDD+). Cette étude s'attache à étudier la REDD+, tant à l'échelle nationale qu'à celle du projet, en fonction de son efficacité à réduire les émissions de carbone et du respect des critères d'efficience, d'équité et des co-bénéfices (les 3E+). Ce guide est accompagné d'un $\mathrm{CD}$ contenant également les instruments de recherche GCS-REDD+ assortis de recommandations techniques qui sont utilisées pour examiner les conséquences des projets de REDD+ sur le plan du bien-être économique et social. Axé sur les projets de REDD+, ce guide jette néanmoins des bases théoriques et propose des méthodes empiriques qui seront valables dans toute une gamme d'interventions dans les domaines de la conservation et du développement.

Pour savoir si l'évolution observée du bien-être économique et social peut être attribuée à des interventions dans le cadre de projets, diverses approches méthodologiques de recherche peuvent être utilisées. Le choix de la méthodologie dépendra $\mathrm{du}$ calendrier du projet, des ressources humaines et financières et de l'influence de l'équipe d'évaluation (cf. Tableau 1). Ce guide décrit ces diverses approches méthodologiques en s'appuyant sur la littérature récente et de plus en plus abondante consacrée à l'évaluation rigoureuse de l'impact des projets de conservation et de développement durable. Nous fournissons un glossaire et une fiche technique (Fiche technique 1) expliquant 
Tableau 1. Possibilités de méthodologies de recherche ayant pour objectif l'évaluation d'impact ex post fondée sur l'évidence empirique

\begin{tabular}{|c|c|c|c|c|c|}
\hline $\begin{array}{l}\text { Commencement } \\
\text { avant le } \\
\text { lancement du } \\
\text { projet? }\end{array}$ & $\begin{array}{l}\text { Intérêt } \\
\text { suscité/ } \\
\text { budget pour } \\
\text { la collecte de } \\
\text { données de } \\
\text { contrôle? }\end{array}$ & $\begin{array}{l}\text { Capacité } \\
\text { d'influer } \\
\text { sur la } \\
\text { conception } \\
\text { du projet? }\end{array}$ & $\begin{array}{l}\text { Méthodologie de } \\
\text { recherche }\end{array}$ & $\begin{array}{l}\text { Élaboration } \\
\text { de scénarios } \\
\text { contrefactuels en... }\end{array}$ & $\begin{array}{l}\text { Nécessité } \\
\text { d'utiliser des } \\
\text { techniques } \\
\text { d'appariement? }\end{array}$ \\
\hline Oui & Oui & Oui & $\begin{array}{l}\text { Randomisation } \\
\text { (Fiche technique 3) }\end{array}$ & $\begin{array}{l}\text { Allocation aléatoire } \\
\text { des sites de projet } \\
\text { et de contrôle }\end{array}$ & Peut-être \\
\hline Oui & Oui & Non & $\begin{array}{l}\text { Avant-après/ } \\
\text { contrôle- } \\
\text { intervention (BACI) } \\
\text { (Fiche technique 4) }\end{array}$ & $\begin{array}{l}\text { Données } \\
\text { d'observation aux } \\
\text { sites de contrôle } \\
\text { avant et après } \\
\text { l'intervention }\end{array}$ & Oui \\
\hline Oui & Non & Non & $\begin{array}{l}\text { Avant-après (BA) } \\
+ \text { projection } \\
\text { de la situation } \\
\text { contrefactuelle } \\
\text { (Fiche technique 5) }\end{array}$ & $\begin{array}{l}\text { Modèles, souvent } \\
\text { fondés sur } \\
\text { les tendances } \\
\text { historiques }\end{array}$ & Peut-être \\
\hline Non & Oui & Non & $\begin{array}{l}\text { Appariement des } \\
\text { unités de contrôle- } \\
\text { intervention (Cl) } \\
\text { (Fiches techniques } \\
5 \text { et } 7 \text { ) }\end{array}$ & $\begin{array}{l}\text { Données } \\
\text { d'observation } \\
\text { (et aussi souvent } \\
\text { recueil de } \\
\text { souvenirs) aux sites } \\
\text { de contrôle après } \\
\text { l'intervention }\end{array}$ & Oui \\
\hline Non & Non & Non & $\begin{array}{l}\text { Autoréférentielle ou } \\
\text { rétrospective } \\
\text { (Fiche technique 6) }\end{array}$ & $\begin{array}{l}\text { Estimation des } \\
\text { " changements dus } \\
\text { au projet » à partir } \\
\text { d'opinions et/ou de } \\
\text { souvenirs }\end{array}$ & Non \\
\hline
\end{tabular}

la terminologie utilisée dans le domaine de l'évaluation d'impact, dans le but d'accroître l'accessibilité de cette discipline à ceux qui travaillent dans le champ de la REDD+. Parmi les concepts essentiels, on peut citer la notion de « situation contrefactuelle », qui s'apparente à l'idée du niveau de référence business-as-usual dans le domaine de la REDD+. Ce concept est central dans les deux domaines : que ce soit pour analyser l'impact causal des projets ou leur additionnalité, il faut comprendre ce qui se serait passé sans projet.

La situation contrefactuelle risque d'être mal définie par une simple comparaison avec les conditions antérieures au projet (parce que d'autres facteurs auraient conduit à des changements même si le projet n'avait pas eu lieu) ou avec des zones ou des usagers de la forêt situés en dehors de la zone du projet (parce qu'ils n'ont probablement pas été inclus dans le projet pour des raisons liées à des caractéristiques fondamentales qui les différencient des autres). Ces comparaisons ont d'ailleurs été dénommées « situations contrefactuelles contrefaites ». Afin d'éviter de faire des analyses contrefactuelles contrefaites, la solution est d'incorporer une méthodologie « expérimentale» dans les projets, en échelonnant les interventions dans le temps et dans l'espace, de manière à ce que la répartition des interventions soit indépendante d'autres facteurs, généralement par la randomisation sous une forme ou une autre. Lorsque c'est possible, on élimine ainsi fort bien les 
explications contradictoires susceptibles de justifier les impacts observés pour ne retenir que ceux qui peuvent être attribués au projet. On peut également recourir aux méthodes "quasi-expérimentales" (dites BACI, BA et CI dans le tableau ci-après), qui reposent sur un plan d'échantillonnage raisonné.

L’ «appariement » est un outil important dans les méthodes quasi-expérimentales. Il s'agit de sélectionner pour les comparer des sites ou des usagers de la forêt qui seront semblables à ceux du projet pour ce qui est de facteurs fondamentaux affectant tant l'inclusion dans le projet que les critères de jugement considérés. Ces facteurs sont appelés "facteurs de confusion », parce qu'ils peuvent porter à confusion en faussant ou en occultant les impacts. Par exemple, si un porteur de projet choisit de travailler avec des villages relativement vulnérables aux changements climatiques (sécheresse ou inondations par ex.). Cette vulnérabilité plus importante peut se traduire, même après le projet, par un bien-être économique et social plus faible que celui d'un échantillon aléatoire de villages avoisinants. Ainsi, au lieu d'utiliser un échantillon aléatoire, nous aurions intérêt à comparer les villages de l'intervention à un échantillon comparable, dont la vulnérabilité initiale aux changements climatiques (avant le projet) est équivalente. Ces observations de « contrôle » seraient intéressantes pour l'élaboration du scénario contrefactuel.

Dans la pratique, il est difficile de mettre en évidence et de mesurer tous les facteurs de confusion ayant une influence sur les zones retenues dans les projets et les résultats des projets dans ces zones. C'est pourquoi la méthode quasiexpérimentale privilégiée est $\mathrm{BACI}$, qui consiste à recueillir des données avant et après le projet, dans des sites de contrôle et d'intervention comparables. Les variations auxquelles sont soumis les critères de jugement peuvent ensuite être comparées d'un site apparié à l'autre, ce qui revient à éliminer l'influence de conditions initiales différentes (puisque seuls les changements depuis le début du projet sont pris en compte) et de changements externes se produisant en même temps que le projet, tels que de nouvelles mesures nationales ou des anomalies climatiques (celles-ci affectant à la fois les sites d'intervention et de contrôle). L'étude
GCS-REDD+ en cours utilise la méthode BACI. Celle-ci demande des ressources importantes pour les prospections sur le terrain, les données quantitatives devant être recueillies avant le projet et sur les sites de contrôle (et non simplement après le projet sur le site d'intervention, conformément à ce qui se pratique habituellement). Il faut également consacrer des ressources à la collecte de données qualitatives pour répertorier tout changement externe affectant seulement les sites d'intervention ou seulement les sites de contrôle et découlant par exemple de facteurs variables dans le temps non pris en compte dans le plan d'échantillonnage, tout comme il faut également allouer des ressources à la représentation de la chaîne causale (abordée ci-après).

Même si ce guide laisse à penser que les méthodologies de recherche peuvent classer les sites et les usagers de la forêt en catégories clairement définies (contrôle ou intervention ou bien avant ou après), la réalité est bien souvent plus complexe. De nombreux projets de REDD+s'inscrivent dans le prolongement d'initiatives de conservation précédentes. Il est donc important d'en donner une description et d'en mesurer l'influence sur les conditions antérieures au projet. C'est la raison pour laquelle il est difficile de définir une date de début de projet. Pour résoudre ce problème, on peut partir du principe que de nombreux projets de REDD+ sont en fait des paquets d'interventions et axer l'évaluation sur une composante particulière de ce paquet, mise en place ou élargie grâce à un financement étroitement lié à la réduction des émissions nettes de carbone. L'évaluation des impacts relatifs des différentes composantes de ce paquet peut également être utile (différentes manières de mettre en œuvre les incitations à l'intention des usagers de la forêt par ex.), plutôt que de se concentrer uniquement sur l'impact global du projet entier.

Par définition, les groupes de contrôle ne doivent pas subir l'influence du projet, c'est-à-dire que la participation d'autres sites forestiers à un projet de REDD+ ne devrait avoir aucune incidence sur les critères de jugement de l'intervention mesurés au niveau des sites de contrôle. Cependant les sites de contrôle doivent être identiques aux sites du projet. C'est pourquoi on recherchera en priorité 
les sites de contrôle dans l'aire la plus proche du projet qui ne présente pas d'interactions avec les usagers de la forêt du site du projet. Entre ces deux endroits se trouveront probablement des usagers de la forêt qui subiront les répercussions indirectes des interventions réalisées dans le cadre du projet. Si l'on dispose de suffisamment de ressources pour l'évaluation, ces usagers intermédiaires pourraient être constitués en un troisième groupe d'échantillonnage, qui pourrait servir à analyser les retombées ou les fuites du projet. Il faudrait au minimum que les usagers de la forêt dans le site du projet soient interrogés sur les activités susceptibles d'avoir des répercussions sur les usagers de la forêt dans d'autres zones, telles que les achats de terres ou les migrations saisonnières de main-d'œuvre.

Dans ce guide, nous tentons de démontrer de manière convaincante la nécessité de collecter des informations sur le processus de mise en œuvre des projets en associant des méthodes qualitatives et quantitatives et en élaborant une chaîne causale (démarche consistant à formuler une théorie du changement). La quantification de la nature et de l'ampleur des impacts sur le bien-être économique et social est nécessaire, mais elle ne suffit pas si l'on veut tirer les enseignements du programme de REDD+. Nous devons également comprendre les phénomènes à l'œuvre sous-tendant les résultats observés et évaluer leurs coûts. Formuler une théorie du changement (mais aussi comprendre celle du porteur de projet) peut fournir un éclairage précieux sur les phénomènes de causalité à l'origine des résultats observés. Le chiffrage des coûts administratifs (liés à la mise en œuvre mais aussi aux coûts de transaction) des projets de REDD+ est essentiel si l'on veut dégager des enseignements de l'analyse des impacts de ces projets. C'est pourquoi il est crucial de faire à chaque fois le lien entre l'objet de notre observation (à savoir le résultat observé) et "pourquoi » on l'observe (à savoir les causes du résultat observé).

La représentation des chaînes causales est une approche itérative. Nous en dégageons les cinq étapes constituantes et les illustrons à l'aide d'exemples. Ce sont : (1) le recensement des caractéristiques du site de REDD+ sur le plan démographique, socioéconomique, biophysique et institutionnel ; (2) la description de l'intervention, en indiquant si sa mise en œuvre s'est déroulée comme prévu ; (3) élaboration d'hypothèses vérifiables en s'appuyant sur la littérature théorique et empirique, et les connaissances des conditions du site ; (4) la détermination des besoins en données quantitatives et qualitatives, nécessaires pour éprouver les hypothèses; (5) la vérification des hypothèses et le réexamen des suppositions de départ faites au sujet des liens de causalité existant entre la mise en œuvre du projet de REDD+ et les changements quantifiables du bienêtre économique et social. La représentation des chaînes de causalité exige de consentir un effort considérable pour comprendre ce qui s'est vraiment passé sur le terrain lors de l'intervention REDD+ et l'influence des activités de REDD+ sur différents indicateurs du bien-être économique des usagers de la forêt, que ce soient des acteurs modestes ou de grands propriétaires terriens. Nous fournissons également des règles pour expliquer l'hétérogénéité des impacts sur les différents usagers de la forêt du site REDD+. Une vision claire de la chaîne causale permet d'expliquer les répercussions contrastées des projets de REDD+ pour les usagers, dont certains tirent des bénéfices sur le plan de leur bien-être économique et d'autres se trouvent au contraire lésés.

Les acteurs du programme de REDD+ doivent prendre en compte plusieurs aspects pratiques lors de la planification de l'évaluation d'impact. Il s'agira donc de respecter des principes d'éthique lors de la recherche en veillant notamment à impliquer les communautés locales dans la conception et le recueil des données, de fournir des informations aux communautés et aux particuliers sur la finalité de la recherche et ses bénéfices potentiels, et de rendre compte des conclusions aux parties prenantes locales. Ils devront également prévoir des crédits suffisants et développer les capacités des ressources humaines dont elles disposent pour l'évaluation d'impact et l'analyse des phénomènes de causalité. Nous soulignons l'importance d'intégrer, avant le lancement du projet, l'évaluation des impacts sociaux dès la conception et la préparation de sa mise en œuvre. L'approche d'évaluation n'en sera que plus souple, et la probabilité que des moyens financiers y soient effectivement consacrés n'en sera que plus grande. Le coût de l'estimation des impacts sociaux se 
justifie par le fait que la viabilité politique et sociale du programme REDD+ et le caractère durable de son rôle dans l'atténuation des changements climatiques dépendent fortement des impacts de ce programme sur les conditions d'existence des populations.

Les projets de REDD+ se déroulent à des niveaux très variés et dans des cadres extrêmement divers. Ils reposent aussi sur toute une gamme d'interventions. Dès lors, il est évident qu'une seule méthode ne pourra être satisfaisante pour analyser les quelque 150 projets de REDD+ proposés ou prévus. Si elle pose des problèmes méthodologiques non négligeables, la variation nous offre la possibilité d'apprendre. Si nous consacrons du temps et des ressources à l'évaluation d'un échantillon représentatif de projets de REDD+ par les procédés les plus avancés, l'application d'une méthodologie de recherche rigoureuse et d'une approche mixte de mise en évidence des chaînes de causalité, et si nous communiquons les conclusions de cette recherche dans les projets et les régions, nous pourrons réellement avoir une influence sur les orientations politiques futures de la REDD+ grâce aux enseignements que nous aurons tirés.

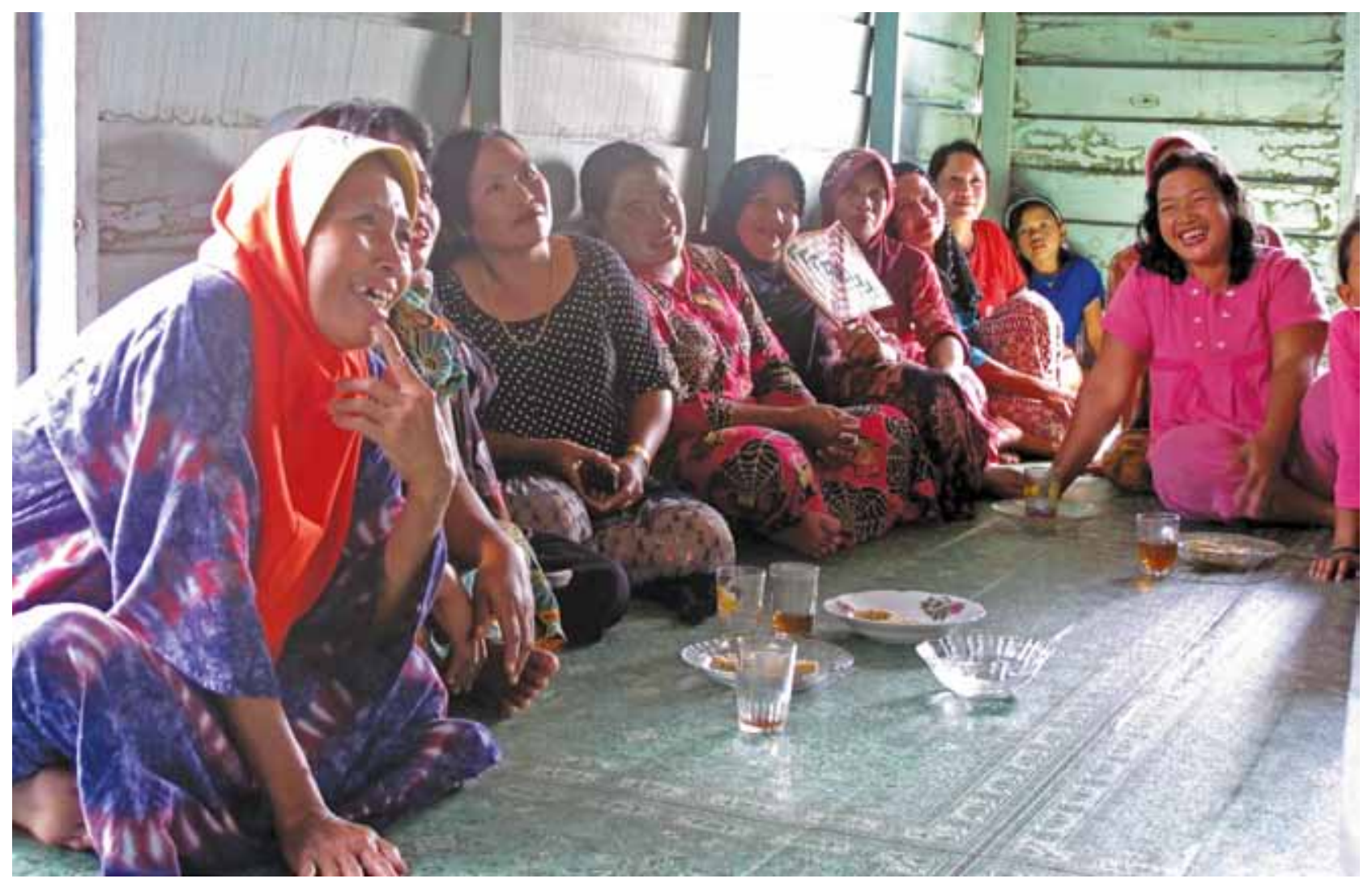

Femmes du village de Galinggang dans le Kalimantan-Centre participant à un entretien de groupe dans le cadre de GCS-REDD+ (৫) Yayan Indriatmoko/CIFOR) 


\section{Préface}

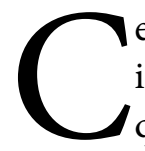

e guide a été conçu à l'usage des acteurs impliqués dans les projets de REDD+, qui sont désireux de s'informer sur les interventions et les conditions susceptibles d'apporter des résultats positifs afin que leurs projets puissent contribuer à améliorer la conception des systèmes de REDD+ au plan mondial. Il vise tout particulièrement ceux qui souhaitent comprendre les répercussions de la REDD+ sur les conditions d'existence dans les forêts tropicales par l'étude des effets de causalité des projets de REDD+ sur le bien-être économique et social. Les informations contenues dans ce guide devraient intéresser les organismes multilatéraux et bilatéraux et les autres donateurs qui financent des projets de REDD+ sous forme de démonstrations et de pilotes. Ces projets natteindront leur but que si une évaluation d'impact rigoureuse est adoptée de manière anticipée, prise en compte dès la conception des projets, et financée intégralement. Le public que nous ciblons comprend également les autorités nationales et régionales qui financent et pilotent les programmes de REDD+, les porteurs de projets, les organisations non gouvernementales et de la société civile qui s'intéressent de près aux impacts de ces projets, ainsi que la communauté scientifique internationale.

Le Centre de recherche forestière internationale (CIFOR), acteur de cette communauté, emploie les méthodes rigoureuses présentées dans ce guide pour tirer les enseignements d'un échantillon de projets de REDD+ répartis sur trois continents : l'Afrique, l'Asie et l'Amérique latine. L'Étude comparative mondiale du CIFOR sur la REDD+ (GCS-REDD+) porte sur 20 sites de projets de REDD+ et son objectif est d'examiner si les projets de REDD+ satisfont aux critères 3E+ (efficacité, efficience, équité et co-bénéfices). Ce projet de recherche englobe toutes les facettes de la mise en œuvre des projets de REDD+, quelles soient de nature socioéconomique ou biophysique.

Nous espérons qu'avec ce guide l'impulsion soit donnée pour que d'autres organisations financent, réalisent ou apportent leur concours à des initiatives de recherche similaires, afin de constituer une base de données scientifiques sur la REDD+ à l'échelle internationale. Nous prévoyons qu'une nouvelle version de ce guide rendra compte des pratiques d'excellence en matière d'évaluation, telles qu’elles auront été développées et éprouvées par l'étude GCS-REDD+ du CIFOR et des travaux menés actuellement par d'autres pour quantifier l'impact causal des projets. C'est avec plaisir que nous recueillerons les réactions à ce guide des parties prenantes engagées dans la mise en œuvre, le financement ou la conduite de telles initiatives

L’objet de ce guide concerne les méthodologies de recherche de l'évaluation d'impact ex post rigoureuse et empirique. Les leçons tirées de ces analyses relèvent du domaine public international ; les connaissances générées actuellement serviront directement à améliorer les projets de REDD+ existants et à venir, et à guider par l'expérience qu'elles permettent d'acquérir l'élargissement du programme de REDD+ aux échelons infranational et national. Si l'étude GCS-REDD+ du CIFOR mesure tant les effets socioéconomiques que biophysiques de la mise en œuvre de la REDD+, ce guide se penche uniquement sur les résultats relatifs au bien-être économique et social, et ce pour deux raisons. D'une part, nous sommes convaincus qu'il existe peu de données dans ce domaine pour alimenter le débat public, surtout comparativement aux recherches actuelles beaucoup plus conséquentes ayant trait au carbone. D'autre part, 
nos méthodes de mesure des résultats biophysiques sur les sites des projets de GCS-REDD+ sont encore au stade de la mise au point et que de ce fait, il est trop tôt pour leur consacrer une place importante dans ce guide.

Nous laissons à d'autres le soin de traiter de la question importante de l'évaluation des impacts sur la biodiversité et les services écosystémiques au plan local, qui pourraient être abordés en tant quéléments à part entière dans les systèmes de mesure, notification et vérification du carbone. Les synergies entre les évaluations de projet, quels que soient les résultats étudiés (effets sur le carbone ou la subsistance des populations), sont évidentes, puisque les résultats dans ces deux domaines dépendent de décisions et de comportements relatifs à l'usage de la forêt. Or, les impacts sur les moyens de subsistance ne sont en général pas étudiés dans le même cadre, ni avec la même rigueur que ceux qui ont une incidence sur l'utilisation du sol ou le carbone.

Nous ne remettons pas du tout en cause le bienfondé d'étudier les résultats carbone de projets dont la finalité est justement d'atténuer les changements climatiques et qui ont été conçus et financés dans cette optique. Cependant nous croyons que la viabilité politique et sociale de la REDD+ tient à une meilleure compréhension et gestion des arbitrages et des synergies à favoriser entre la réduction des émissions et l'amélioration du bien-être économique et social. Notre approche est validée par la popularité dont jouissent les normes pour la conception de projets se rapportant au climat, aux communautés et à la biodiversité (standards CCB) et par l'intérêt suscité par les nouvelles normes sociales et environnementales de la REDD+, portées par l'Alliance Climat, Communautés et Biodiversité (CCBA) et CARE.

À notre avis, le rôle des projets et programmes de REDD+ dans l'étude des impacts sociaux doit être reconnu, outre leur finalité affichée. Ils permettent en effet de conduire des recherches dans lesquelles auront été intégrées des méthodologies d'évaluation expérimentale et quasi-expérimentale et de financer la collecte de données en satisfaisant aux critères les plus exigeants s'agissant de la recherche de preuves de causalité. En plus de l'évaluation des résultats sur le plan du bien-être collectif, nous formulons des recommandations pour représenter l'enchaînement des causes et des effets reliant l'intervention de REDD+ à ses résultats. Par la quantification des impacts et l'étude de leurs causes, les évaluateurs peuvent développer notre compréhension relativement limitée des facteurs qui favorisent des résultats sociaux positifs dans le cadre d'initiatives de conservation et de développement telles que la REDD+. Ceci permettra ensuite d'inspirer et de compléter les travaux parallèles consacrés aux méthodes de validation et de vérification des projets par rapport aux référentiels des marchés volontaires et de tout futur marché d'engagements contraignants.

Pour résumer, ce guide est surtout pertinent pour les acteurs de la REDD+ qui sont :

- attachés à l'utilisation de plans expérimentaux et de méthodes de recherche rigoureux pour mieux connaitre les résultats des projets de REDD+ sur le plan du bien-être économique et social ;

- disposés à analyser les impacts causaux ex post des projets de REDD+ comparativement à ce qui se serait passé en l'absence de ces projets (scénario parfois distinct des projections de niveaux de référence pour l'attribution de crédits) ;

- intéressés par la comparaison et la mise à l'épreuve d'approches différentes en matière d'analyse des impacts sociaux (méthodes généralement utilisées pour la vérification dans le cadre des normes de compensation de carbone sur les marchés volontaires, telles que le référentiel CCBA), afin d'en dégager la validité commune ;

- sensibilisés à l'intérêt de mieux comprendre s'il existe un arbitrage à faire ou des synergies à exploiter entre l'amélioration du bienêtre économique et social au plan local et la réduction des émissions de carbone au plan mondial, et si oui dans quels domaines ;

- prêts à affecter des ressources afin de contribuer au capital mondial de connaissances sur la mise en œuvre des projets de REDD+. 



\section{Tirer les enseignements de la REDD+ : un exercice nécessaire}

\subsection{Pourquoi est-il nécessaire de tirer les enseignements des projets de REDD+ ?}

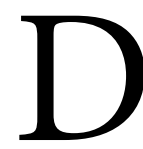

epuis la Conférence des Parties de

Bali (COP 13) en décembre 2007,

environ 150 projets ont été prévus qui ont pour objectif la réduction des émissions dues à la déforestation et à la dégradation des forêts et la promotion de la conservation, de la gestion durable des forêts et du renforcement des stocks de carbone forestier dans les pays en développement ; ces projets sont communément appelés les « projets de REDD+ » (cf. Encadré 1). ${ }^{1}$ Laffectation de crédits et de ressources supplémentaires à la REDD+, et en particulier à ces projets, constitue l'un des rares résultats concrets de la $\mathrm{COP} 15$, réunie à Copenhague en 2009 (Climate Funds Update 2010 ; Coria et al. 2010). La réputation de la REDD+ comme un moyen relativement rapide, facile et à bas coût de ralentir les changements climatiques est à l'origine de ce succès, ainsi que l'atteste la formule souvent répétée "nous savons comment NE PAS couper les arbres ». De plus, la REDD+ suscite aussi l'adhésion parce qu'elle est perçue comme un moyen d'attirer des crédits de financement pour la conservation des forêts tropicales riches

\footnotetext{
1 Nous avons répertorié des propositions ou des plans correspondant à environ 300 projets de carbone forestier. Environ la moitié d'entre eux semblent être axés uniquement sur le boisement/reboisement. Parmi les 150 projets restants qui pourraient devenir des projets REDD+, beaucoup n'en sont quaux toutes premières étapes de planification. Cependant, des dizaines de projets avancent sur la voie de la mise en œuvre, notamment au Brésil, au Pérou et en Indonésie (Sills et al. 2009 ; Wertz-Kanounnikoff et Kongphan-apirak 2009).
}

en biodiversité et de générer un nouveau flux de revenus dans ces régions pour les populations pauvres vivant en milieu rural.

Mais le programme de REDD+ (au niveau national et du projet) est toujours loin de faire l'unanimité, ses détracteurs craignant qu'il fournisse une échappatoire se traduisant par moins de réductions d'émissions nettes dans le monde, accentue les inégalités existantes, fragilise les droits déjà précaires des populations dépendantes des forêts les plus démunies, et détourne une partie des financements dont aurait pu bénéficier la biodiversité (Sunderlin et al. 2009; SpringateBaginski et Wollenberg 2010).

Les projets de REDD+ peuvent fournir des preuves pour informer ces débats. ${ }^{2}$ Ils offrent l'occasion unique de mieux comprendre les répercussions des différentes formes d'interventions sur les

\footnotetext{
2 Les projets ne constituent qu'un des moyens de rassembler des preuves et d'accroître nos connaissances. Comme le montrent Angelsen et al. (2008) et Angelsen et al. (2009), les projets de REDD+ ne permettent pas de tirer tous les enseignements : par exemple, ils ne peuvent pas montrer quels sont les impacts des changements de politiques nationales. De plus, les problèmes de mesure et de fuites inhérents à l'approche de projet (Richards et Andersson 2001) constituent une source d'imprécision quand il s'agit de déterminer les retombées sur la forêt et le bien-être économique dans les évaluations d'impact des projets. Cela limite la portée des enseignements qui ne peuvent être transposés aux programmes nationaux couvrant un territoire plus étendu. Comme le souligne l'encadré 4, l'Étude comparative mondiale du CIFOR sur la REDD+ comporte des volets axés sur les systèmes et stratégies politiques nationales et sur la modélisation et le suivi des niveaux de références nationaux, parallèlement à l'étude des projets de REDD+ et de leurs acquis.
} 


\section{Encadré 1. Répartition mondiale des projets de REDD+}

Depuis la COP 13 de la CCNUCC, les projets visant à réduire les émissions liées à la déforestation et à la dégradation des forêts (REDD) suscitent un regain d'intérêt. Ces projets mettent à profit les résultats antérieurs de projets de « déforestation évitée » (Caplow et al. sous presse). Ils comprennent des initiatives bilatérales ayant pour objectif de renforcer les capacités et de réformer les politiques nationales dans les pays hôtes. Ils cherchent aussi à générer des crédits carbone réels, additionnels et vérifiables, afin de les mettre en vente sur le marché volontaire. Certains poursuivent sur la lancée des projets de séquestration du carbone par le boisement/reboisement $(B / R)$, développés initialement dans le cadre du Mécanisme pour un développement propre (MDP) et les marchés de compensation volontaires, de sorte qu'en pratique, il n'y a pas de différence nette entre les projets de REDD+ (comprenant la gestion et la restauration) et les projets de BR. Leurs interventions varient notamment de paiements versés directement et individuellement à des propriétaires forestiers et usagers (cas plus fréquent en Amérique latine) à la prévention de la déforestation ou de la conversion en plantations à grande échelle (cas plus courant en Indonésie) en passant par la gestion communautaire des forêts (souvent dans les projets africains). De nombreux projets de REDD+ poursuivent les activités de conservation antérieures dans des endroits particuliers. Certains projets visent aussi à améliorer l'aménagement de l'espace dans de vastes paysages et sa mise en application, souvent en collaboration avec les collectivités locales. Toute cette palette de projets de REDD+ nous offre l'occasion d'apprendre à tirer parti du potentiel d'atténuation des changements climatiques des forêts. Dans ce guide nous entendons par « projet de REDD+ » toute initiative visant à réduire, de manière directe et quantifiée, les émissions de carbone nettes issues d'une région forestière ou d'un paysage infranational définis. Comme le montrent Sills et al. (2009) et Cerbu et al. (2009), ces projets ont une distribution géographique très étendue, mais inégale d'une région boisée à l'autre dans les pays en développement (cf. Figure 1). Le Brésil et I'Indonésie en particulier accueillent un grand nombre de projets en raison de leurs stocks importants de carbone forestier. En revanche, du côté de I'Afrique, la République démocratique du Congo possède peu de projets malgré la taille de son stock de carbone forestier. La Tanzanie, quant à elle, compte un nombre croissant de projets, financés principalement par l'État norvégien (Norvège 2010). Les porteurs de projet dans toutes les régions cherchent à faire certifier leurs projets, selon des référentiels de carbone, tels que la Norme de compensation volontaire de carbone et les Standards de conception de projets climat, communautés et biodiversité (CCB), dont les critères exigent la preuve de l'existence de co-bénéfices pour la biodiversité et les communautés locales. Le succès engrangé et le prix plus élevé obtenu par la certification CCB (Ecosecurities 2010) justifient I'importance accordée à l'analyse des résultats des projets de REDD+ sur le plan du bien-être économique.

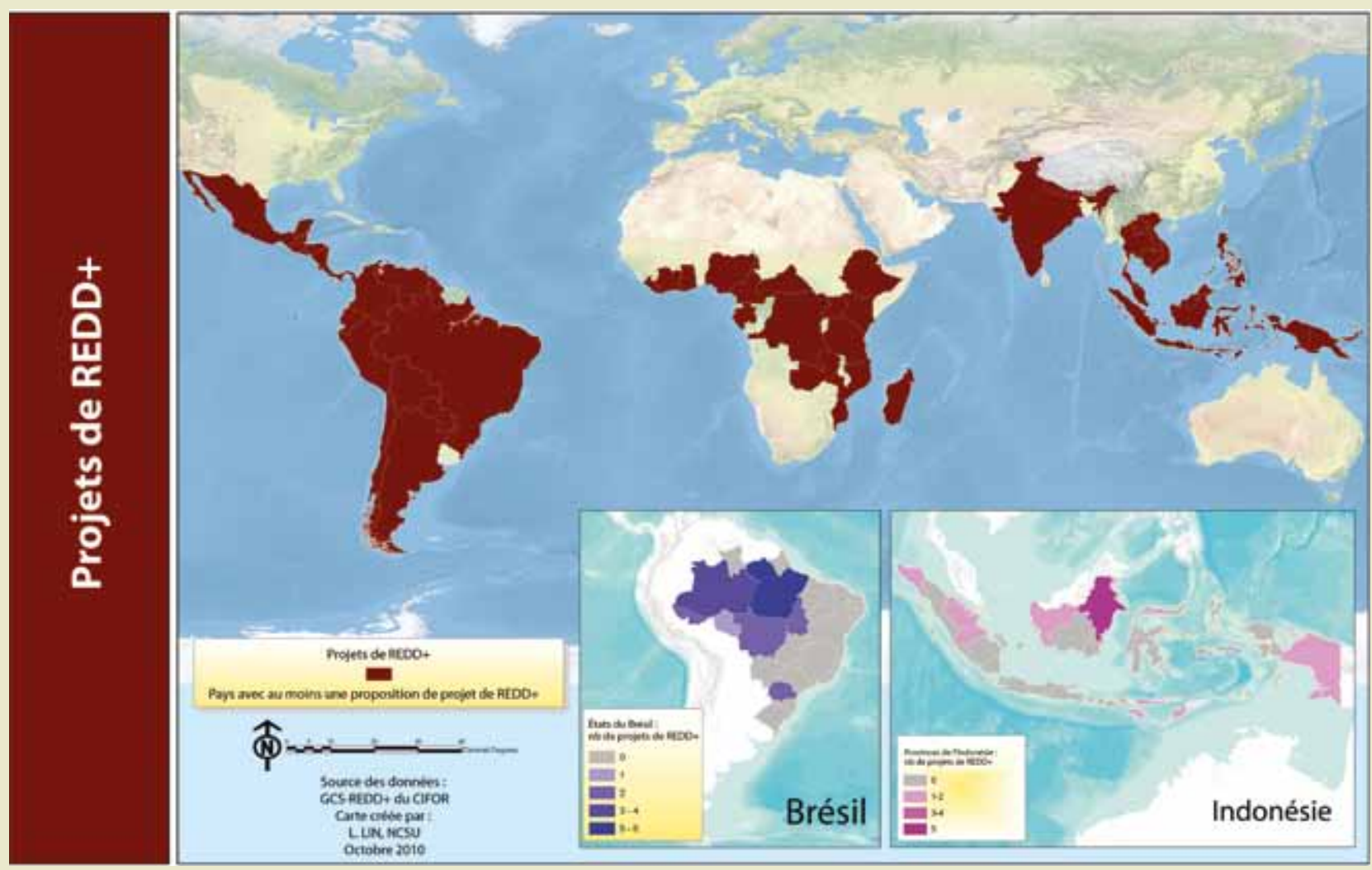

Figure 1. Répartition mondiale des projets de carbone forestier 


\section{Encadré 2. Pourquoi il est possible de tirer les enseignements des projets de REDD+}

Les projets de REDD+ fournissent l'occasion inégalée d'exploiter les acquis, et ce pour les raisons suivantes :

- répartition mondiale et calendrier relativement synchrone des projets ;

- allocation de ressources financières considérables pour l'élaboration, la mise en œuvre, le suivi et l'évaluation ;

- objectif clairement affirmé de capitalisation des acquis fixé par les négociateurs internationaux;

- accent mis sur la « conditionnalité » et l'« additionnalité », critères qui correspondent à ceux des systèmes d'évaluation d'impact, et qui de ce fait facilitent cette dernière ;

- mise en œuvre des projets concentrée dans certaines régions bien définies géographiquement, ce qui rend possible la comparaison entre régions (mais qui est plus difficile dans le cas des politiques nationales);

- probabilité que de nombreuses questions non résolues portant sur les synergies et arbitrages éventuels entre le carbone et les impacts sur les moyens d'existence se manifestent à l'échelle du projet ;

- possibilité de tirer parti des progrès rapides récents réalisés dans le domaine des méthodes d'évaluation d'impact et des analyses de causalité, développées et appliquées pour la plupart dans d'autres secteurs politiques, mais qui sont transférables aux projets et programmes de conservation, tels que la REDD+.

forêts, mais également sur les populations qui y vivent, les gèrent et en dépendent. Les projets de REDD+ ressemblent aux initiatives antérieures de conservation des forêts à de nombreux points de vue (Blom et al. 2010), mais ils s'en démarquent aussi par les nouvelles perspectives et les nouveaux défis créés, et en particulier par leur orientation sur les résultats (Encadré 2). Les porteurs de projet s'attendent et se préparent à surveiller et évaluer les changements d'utilisation des terres et d'émission de carbone, qui sont étroitement liés à leur financement, mais qui ont aussi des conséquences réelles pour les populations locales. Il existe donc à la fois le besoin et l'opportunité de réaliser une analyse rigoureuse des causes des impacts sur les moyens d'existence. Nous disposons donc d'un créneau limité, mais décisif, pour jeter les bases d'un travail d'évaluation répondant à ces exigences, par le choix d'un concept expérimental, la description approfondie de la sélection des sites et des autres décisions relatives à la mise en œuvre et la collecte de données initiales à partir d'échantillons rigoureusement sélectionnés de participants et de non participants. En appliquant une méthodologie de recherche rigoureuse à de nombreux projets obéissant à des stratégies diverses et qui sont réalisés dans des conditions différentes, nous serons en mesure de voir ce qui marche et ce qui ne marche pas, et d'orienter le développement de nouveaux projets de REDD+ : au final, nous serons en meilleure posture pour élargir ou inclure les projets de REDD+ dans les systèmes nationaux et régionaux.
Les projets de REDD+ présentent bon nombre de points communs avec les projets de conservation et de développement qui tentent d'influencer ou de contenir le comportement des usagers des ressources forestières, quelle que soit leur taille, avec pour objectif l'amélioration des conditions environnementales. Dans le cas de la REDD+, l'objectif clairement affiché est de réduire la déforestation et la dégradation des forêts, ou de favoriser la restauration, la réhabilitation et la conservation de façon à diminuer en même temps les émissions nettes de carbone. Pour y parvenir, les projets se dotent d'un plan, «la représentation de la chaîne de causalité », qui exige habituellement des populations locales quelles modifient la façon dont elles subviennent à leurs besoins, soit parce que les scieries offriront moins d'emplois ou que de nouvelles pratiques agricoles seront adoptées qui ne recourent pas aux feux de brousse pour le défrichement de nouvelles terres (cf. Annexe B.4 qui présente une liste sélective de textes publiés sur les moteurs de la déforestation et de la dégradation). Dans de nombreux projets de REDD+, ces changements ont pour but d'avoir des effets positifs nets sur le « développement » ou les conditions d'existence dans la localité du projet. En outre, nombreux sont les porteurs de projet qui souhaitent chiffrer et recevoir des crédits pour leur contribution au renforcement des moyens de subsistance des usagers locaux des forêts via la certification conformément aux référentiels élaborés par des organisations comme l'Alliance Climat, Communautés et Biodiversité (CCBA) ou 


\section{Encadré 3. Normes et systèmes de certification pour les projets de REDD+}

Le marché volontaire de compensation de carbone est le seul lieu de vente des crédits carbone générés par les projets de REDD+. Ce « marché » comprend en réalité toute une gamme de bourses, de courtiers et d'acheteurs directs. Hamilton et al. (2010) font état de $2846 \mathrm{ktCO}_{2}$ e de crédits de REDD+ échangés en 2009, total nettement supérieur aux $730 \mathrm{ktCO}_{2}$ e de 2008, mais qui ne représente toujours qu'une part de marché très faible. Les acheteurs sont souvent motivés par la responsabilité sociale d'entreprise ou souhaitent se positionner sur les futurs marchés d'engagements contraignants. Quoi qu'il en soit, ils recherchent des garanties que les crédits achetés réduisent réellement les émissions nettes de carbone, sans répercussions négatives pour la biodiversité et les moyens d'existence des populations locales. On dénombre à I'heure actuelle plus d'une douzaine de référentiels et systèmes de certification élaborés pour fournir ces garanties. Seuls certains d'entre eux certifient les projets de REDD+ (CORE 2010) : ils s'attachent à apporter des solutions aux questions d'additionnalité, de permanence et de fuite qui continuent de se poser s'agissant de la REDD+, tout en examinant les impacts sur la population locale et l'environnement.

Le référentiel dominant est la Norme de compensation volontaire de carbone (VCS), dont se réclamait plus d'un tiers du total des crédits échangés sur le marché volontaire en 2009 (Hamilton et al. 2010, VCS 2010). Ce référentiel est axé sur l'intégrité des réductions d'émissions, comprend une analyse de risques indépendante et exige le versement de crédits dans un compte tampon commun. L'association VCS a mis en place des partenariats avec trois registres qui suivent l'évolution des VCU (Unités de crédit carbone volontaires) vérifiées par son intermédiaire. De nombreux projets de REDD+ ont l'intention de faire certifier leurs crédits selon le référentiel VCS (Ecosecurities 2010). Dans ce but, ils sont tenus d'élaborer, puis d'obtenir un double agrément, pour les méthodologies qu'ils souhaitent mettre en place visant à déterminer des niveaux de référence, tenir compte des fuites, et surveiller, faire rapport et vérifier les activités liées au sol et les émissions. L'agrément des méthodologies s'est avéré être un goulet d'étranglement non négligeable. En août 2010, I'association n'avait approuvé qu'une seule méthodologie avec pour objet la déforestation programmée évitée, de nombreuses autres relatives à la déforestation et à la dégradation non programmées dans les zones frontalières et dans les mosaïques étant toutefois en phase d'examen. Le registre américain du carbone (ACR) a lui aussi proposé une méthodologie de REDD+, soumise à l'examen et aux commentaires du public en août 2010 (ACR). L'Alliance Climat, Communautés et Biodiversité (CCBA 2010) développe également des référentiels faisant autorité qui régissent les projets de REDD+. La CCBA gère un registre de projets certifiés conformément à ses référentiels, mais ne délivre pas de crédits d'émissions certifiés. La CCBA n'approuve pas de méthodologies particulières, mais a participé à la création d'un nouveau manuel qui fournit des informations sur les méthodes peu onéreuses pour l'analyse des bénéfices sociaux des projets de carbone forestier (Richards et Panfil 2010). À l'origine, les standards CCB ont été conçus pour permettre de faire ressortir les projets de qualité respectant les droits des populations locales et leur apportant des bénéfices, tout en préservant la biodiversité. Ils sont devenus essentiels depuis pour I'accès au marché et la crédibilité des projets ; ainsi, de nombreux porteurs de projets REDD+ se donnent pour objectif de satisfaire aux critères des standards CCB, sans nécessairement avoir l'intention de vendre des crédits (Madeira et al. 2010). Selon une étude de marché récente, de nombreux acheteurs sont prêts à payer plus cher les projets possédant les deux certifications, VCS et CCB (Ecosecurities 2010).

L'alliance CCBA travaille également avec CARE au développement et au pilotage des Standards sociaux et environnementaux de REDD+, destinés aux programmes nationaux de REDD+ cherchant à faire la preuve de leurs « co-bénéfices » sociaux et environnementaux.

Il existe aussi d'autres normes conçues pour s'ajouter aux normes de comptabilité du carbone, telles que le référentiel Social Carbon, mais leur couverture est beaucoup plus limitée. Enfin, certaines normes cherchent à traiter aussi bien de la comptabilité du carbone que des bénéfices sociaux; ; cest le cas de la norme Plan Vivo. 
Plan Vivo (Encadré 3). Le défi pour les spécialistes de la REDD+ est le même que celui auquel doit faire face le milieu plus vaste de la conservation et du développement : comment savoir si les changements qui s'opèrent dans l'environnement (carbone) ou le bien-être sont le résultat direct de l'intervention dont ils ont eu l'initiative? Quand peuvent-ils s'attribuer la paternité de la réussite du projet ou au contraire la responsabilité de son échec?

L'évaluation REDD+ est fréquemment réalisée à l'aide du cadre de référence des $3 \mathrm{E}+$, qui s'articule autour des critères d'efficacité, d'efficience, d'équité et des co-bénéfices (Angelsen 2009). Les efforts méthodologiques les plus importants semblent avoir été consacrés à la mesure du premier «E», c'est-à-dire la détermination des impacts sur les émissions nettes de carbone ; cf. l'Annexe B.5 qui répertorie les références et les ressources portant sur la mesure, la notification et la vérification $(\mathrm{MNV}) \mathrm{du}$ carbone. D'autres initiatives s'attachent à utiliser les résultats de la $\mathrm{MNV}$ du carbone pour en déduire les impacts sur les autres services écosystémiques et la biodiversité (Teobaldelli et al. 2010). Ce guide étudie les méthodes de mesure de l'impact des projets de REDD+ sur les deux autres $\mathrm{E}$, c'est-à-dire les impacts (coûts et bénéfices) sur les populations locales qui sont imputables aux projets. Nous nous concentrons sur les méthodes d'évaluation d'impact et les plans expérimentaux rigoureux qui fournissent des preuves empiriques pour appuyer leurs analyses contractuelles; ces analyses permettent de se faire une idée ex post de ce quaurait été l'évolution du bien-être économique et social si l'intervention REDD+ n'avait pas eu lieu.

Le Centre de recherche forestière internationale (CIFOR) a lancé l'Étude comparative mondiale sur le programme de REDD+ (GCS-REDD+), qui s'est donné comme objectif de relever le défi de l'analyse rigoureuse des projets de REDD+ (cf. Encadré 4). Ce guide expose la méthodologie et les outils de recherche utilisés dans le « Volet 2 » de l'étude GCSREDD+ pour quantifier les impacts des projets sur le bien-être collectif, espérant ainsi favoriser et faciliter la reprise de cette approche parmi les autres acteurs de la REDD+ afin de contribuer à la construction de la base internationale de données scientifiques sur la REDD+. Ce guide présente en même temps une gamme de méthodologies de recherche avec différentes exigences en matière de collecte de données et d'analyse statistique. Nous examinons le raisonnement qui caractérise chacune des méthodologies, les données nécessaires, ainsi que les questions budgétaires, de ressources humaines et d'éthique qu'elles posent.

L'évaluation d'impact quand elle est rigoureuse ${ }^{3}$ permet de quantifier l'importance des effets de causalité d'un projet de REDD+, en nous informant non seulement sur ce qui se passe du fait d'un projet, mais aussi sur les raisons expliquant ce que nous observons. Les méthodes qualitatives, comprenant des observations et des entretiens très poussés sur le terrain sont importantes pour le choix des critères de jugement et l'interprétation des effets estimés. Il est également essentiel de chiffrer les coûts administratifs des projets (coûts de mise en œuvre et d'opération) si l'on veut tirer les enseignements des impacts des projets. Dans ce guide, nous présentons une approche reposant sur une méthodologie mixte permettant d'élaborer et de mettre à l'épreuve « une représentation de la chaîne de causalité » des impacts des projets de REDD+ sur le plan du bien-être économique et social.

\subsection{Pourquoi faut-il évaluer l'impact des projets sur le bien-être économique et social ?}

Le plan d'action de Bali comporte une exigence de mesure des changements d'émissions nettes de carbone résultant des activités des projets de REDD+. La communauté scientifique et les initiateurs de projets se sont donc vus obligés de se familiariser avec la notion des scénarios contrefactuels (généralement appelés « niveaux de référence » dans le milieu de la REDD+, comme l'explique la Fiche technique 1). L'estimation de l'évolution des émissions de carbone si le projet

\footnotetext{
3 Si dans ce guide nous utilisons le terme "évaluation " au sens large, l'expression "évaluation d'impact" fait référence à un ensemble bien précis de plans expérimentaux et de méthodes permettant d'analyser et de comprendre les résultats de politiques, de programmes et de projets. L'évaluation d'impact, également appelée évaluation de programme, s'attache à quantifier les effets et à déterminer dans quelle mesure ces effets peuvent être imputés au programme et non à d'autres causes (Khandker et al. 2010). Se reporter à la Fiche technique 1 pour une présentation détaillée de la terminologie utilisée dans le cadre de l'évaluation d'impact et de la REDD+.
} 


\section{Encadré 4. Étude comparative mondiale du CIFOR sur la REDD+}

Des informations actualisées sur cette étude comparative sont disponibles sur le site www.forestsclimatechange.org.

La réalisation de la REDD+ demande de nouvelles connaissances et de nouvelles compétences.

- Étant donné l'urgence due aux changements climatiques et la nécessité de fournir rapidement des informations, le CIFOR analysera les politiques et les pratiques de REDD+ et en diffusera les données à un public international.

- Le CIFOR entend créer des outils efficaces et efficients pour œuvrer à la réduction des émissions de carbone forestier et à la production de cobénéfices tels que le soulagement de la pauvreté et la préservation de la biodiversité.

- L'objectif de cette étude est d'influer sur la conception des projets de REDD+ à trois niveaux :

- Local : projets sur site et paysage, la surveillance étant assurée par la population.

- National : formulation de politiques, y compris de scénarios de niveaux de référence nationaux. - International : architecture de la REDD+ dans le cadre de l'accord post-2012 sur la protection du climat.

Le CIFOR travaille avec un vaste réseau de partenaires : porteurs de projets, décideurs et négociateurs, qui bénéficieraient de recommandations et de réflexions relatives à leurs propres activités et à celles entreprises dans le cadre d'autres projets dans le monde.

- Tout au long des quatre années que durera cette initiative, le CIFOR fournira des informations utiles à la conception des projets REDD+ dans la période précédant 2012 et à leur mise en œuvre à partir de cette année charnière.

- Ce travail est divisé en quatre volets interdépendants et sera mené dans neuf pays répartis en Amérique latine, en Asie et en Afrique. Des conférences et des ateliers seront organisés chaque année pour favoriser les échanges d'idées et faire le bilan. Des publications seront diffusées afin d'assister la mise en œuvre de la REDD+.
Volet 1 : Processus et politiques de REDD+ au plan national

- Analyse des processus de la REDD+ de première génération en utilisant des stratégies rigoureuses, comprenant notamment l'analyse des discours médiatiques, l'étude des réseaux liés aux politiques et l'évaluation par score des stratégies employées, afin de garantir la qualité des résultats

- Analyse de la prise en compte des différents intérêts à tous les niveaux dans les processus nationaux de formulation des politiques de REDD+

- Vérification du respect du principe des critères 3E+ dans les résultats de ces processus

Volet 2 : les sites des projets de REDD+

- Collecte de données avant et après la mise en œuvre des interventions dans 20 sites de projets de REDD+, couvrant des villages directement concernés et des villages témoins

- Création d'une base de données internationale en ligne sur la REDD+ à partir des résultats de cette campagne ambitieuse de collecte de données

- Production d'un manuel du praticien ayant pour thème le bilan de la REDD+ après la première année d'étude afin de satisfaire au mieux aux critères de $3 \mathrm{E}+$

Volet 3 : Surveillance et niveaux de référence

- Perfectionnement des méthodes de détermination des niveaux d'émission de référence pour aider les pays à calculer au mieux leurs fourchettes futures d'émissions

- Mise à disposition d'un plus grand nombre de facteurs d'émissions pour la mise en œuvre des méthodes du GIEC pour la comptabilisation nationale des gaz à effet de serre

- Elaboration de méthodes adaptées pour que les mesures puissent être réalisées par les populations

Volet 4 : Partage des connaissances

- Préparation d'une stratégie de partage des connaissances

- Mise en place d'une communauté en ligne souhaitant approfondir ses connaissances par la création d'un site Web interactif

- Echange d'informations à l'occasion d'événements et de conférences de grande portée

- Utilisation novatrice des médias afin d'intéresser les journalistes d'horizons divers 
n’avait pas eu lieu est déterminante pour savoir si ce projet a été à l'origine d'une réduction supplémentaire des émissions de carbone. Le fonctionnement de la REDD+ repose sur des incitations : cette approche contrefactuelle est donc nécessaire pour comprendre les répercussions des projets sur les taux de carbone.

Nous partons du principe que les impacts sur les émissions de carbone doivent être remis à leur juste place en les associant à une estimation non moins rigoureuse des impacts dans les autres domaines, en particulier le bien-être des populations vivant et travaillant dans la zone du projet. Il est en effet crucial d'analyser les impacts sur le bien-être économique des populations locales si l'on veut comprendre les conséquences sociales plus larges de la REDD+ et sa viabilité politique à longue échéance. Dans un avenir plus immédiat, les porteurs de projet, donateurs et organismes de certification tels que la CCBA et l'association de la Norme de compensation volontaire de carbone (VCS) doivent connaître les résultats des projets qu'elles certifient et les compromis effectués entre la conservation et les conditions d'existence des populations pour obtenir ces résultats. Nous pensons que la réussite ou l'échec de la REDD+ à tous les niveaux dépendra de notre aptitude à concevoir des interventions qui, dans le pire des cas, ne portent pas préjudice aux populations locales, mais qui, dans le scénario de réussite, conduisent à des résultats favorables tant sur le plan des réductions d'émissions de carbone que sur celui des conditions d'existence en milieu rural.

Dans ce guide, nous définissons le « bien-être économique et social » comme comprenant tout un ensemble de facteurs influant sur le bien-être humain. La méthode de l'étude GCS-REDD+ est axée sur les revenus des ménages (comprenant les revenus en liquide et en nature), leur composition (part des revenus d'origine agricole, forestière ou autre), la description et les raisons de leur évolution. Pour expliquer pourquoi l'on observe ou non certains impacts, l'étude GCS-REDD+ porte une attention particulière à la possibilité pour les populations locale d'avoir voix au chapitre et de prendre en main leur destin (leur participation plus ou moins grande dans les processus d'autorisation, de conception et de mise en œuvre de la REDD+), aux connaissances (dans la mesure où celles-ci sont déterminantes pour le critère précédent), au rôle joué par les différences hommes-femmes (les revenus et possibilités d'emploi et de subsistance, les droits de propriété ainsi que les possibilités d'expression et les connaissances étant conditionnés par l'appartenance sexuelle dans la plupart des cultures), et au régime foncier (les droits de propriété sur les terres et les ressources influant largement sur l'issue positive ou négative des interventions de la REDD+).

Pour quantifier les impacts des projets sur le bienêtre collectif et éliminer les explications d'une autre nature ou contradictoires, les plans expérimentaux les plus intéressants consistent à collecter des données antérieures et postérieures au projet, à l'intérieur et à l'extérieur des sites de ces projets. Cette démarche a un coût plus élevé que l'approche permettant de satisfaire les critères actuels des référentiels du marché volontaire du carbone. Pour l'étude GCS-REDD+, ces coûts seront largement amortis par les connaissances relatives à la REDD+ en général et aux projets de REDD+ en particulier qu'elle permet de générer. L'évaluation d'impact rigoureuse fournira des preuves tangibles permettant de mettre un terme aux controverses à propos des conséquences de la REDD+ sur les conditions d'existence des populations. Appliquée à une gamme d'interventions dans des conditions très différentes et associées à une méthodologie mixte d'analyse des causes et des effets, l'évaluation d'impact permettra de perfectionner les projets actuels et de déterminer les meilleures pratiques à appliquer dans le cadre des projets à venir. En outre, l'évaluation d'impact pourra servir à valider et affiner les méthodes ex ante de prévision des résultats dans les zones des interventions et correspondant aux niveaux de référence, tout en fournissant des intrants plus fiables pour la modélisation des impacts de la REDD+ à très longue échéance.

\subsection{Quels projets faut-il évaluer ?}

L'évaluation d'impact rigoureuse, surtout celle qui s'appuie sur les méthodologies optimales de recherche a un coût élevé en raison du travail de conception important, du budget nécessaire pour la collecte des données et des compétences en 
statistique requises pour l'analyse de ces dernières. Il ne faut donc pas nécessairement soumettre tous les projets de REDD+ à ce type d'évaluation dans le but d'estimer leurs impacts sur le bienêtre collectif. Mais alors, qui doit investir dans de telles analyses et quels projets doivent être retenus pour l'évaluation?

Pour certains porteurs, ces méthodes seront utiles pour assurer la surveillance et la gestion de leur portefeuille de projets ou de programmes régionaux (par ex. les initiatives officielles infranationales créées pour satisfaire les critères des normes sociales et environnementales de la REDD+). Des preuves concrètes des effets de causalité éviteront aux porteurs de projet de porter le chapeau pour des résultats qui ne sont pas la conséquence de leurs projets ou d'avoir à faire face à des attentes dépassant le cadre des projets. Ils pourront ainsi mieux se concentrer sur les résultats (bons ou mauvais) qui sont vraiment de leur ressort, car effectivement causés par les interventions de REDD+. L'évaluation d'impact rigoureuse s'appuyant sur des méthodes à validité interne élevée fournit des bases solides pour rendre compte des projets et des programmes de REDD+ et les perfectionner.

Cependant, elle offre surtout la possibilité de tirer les enseignements de chaque projet pour contribuer à la conception des systèmes de REDD+ futurs. De manière plus évidente, l'évaluation rigoureuse des premiers projets permettra de décider des interventions les plus aptes à un élargissement infranational ou national, en évitant ainsi d'investir des ressources supplémentaires en interventions infructueuses (ou néfastes sur le plan du bienêtre économique et social). La base de données scientifiques tirée de l'évaluation rigoureuse des premiers projets pilotes et de démonstration peut permettre de s'armer face aux attentes et servir de base pour les ajustements.Cela évitera peut-être ainsi à la REDD+ de tomber dans l'écueil de la promotion cyclique exagérée en vogue dans les milieux de la conservation et du développement, qui suscite des attentes irréalistes (cf. Skutsch et McCall 2010). C'est pourquoi l'évaluation rigoureuse des projets de REDD+ se situe en parfaite adéquation avec la mission que se sont donné diverses organisations bilatérales, multilatérales et internationales, laquelle consiste à élaborer des stratégies efficaces d'atténuation des changements climatiques.

Dans le but d'atteindre cet objectif plus général, les projets de REDD+ doivent aussi posséder une certaine validité externe en générant des enseignements transférables. On suppose donc que l'évaluation doit d'abord privilégier les interventions susceptibles de subir un élargissement à des zones représentatives d'un paysage entier ou des groupes représentatifs de la population générale. De plus, les évaluations de projets dont les porteurs communiquent volontiers leurs coûts de mise en œuvre seront les plus utiles quand il s'agira d'analyser les arbitrages et les complémentarités dans tous les domaines couverts par les critères des $3 \mathrm{E}+$. Enfin, les porteurs de projet disposés à recourir à un plan expérimental, à une mise en œuvre progressive et à la vérification des différentes options sur des sous-échantillons sélectionnés indépendamment de leurs autres caractéristiques, sont susceptibles de pouvoir faire preuve d'une validité interne et externe élevée ; ceci, à condition cependant que les interventions ou les options de mise en œuvre vérifiées aient une portée générale et que «les expériences » puissent être répétées dans d'autres projets.

Les donateurs et programmes de financement soutenant un grand nombre de projets de REDD+ pourraient jouer un plus grand rôle dans cette capitalisation des acquis en définissant les points essentiels qui caractérisent les interventions et les conditions favorables à l'obtention de résultats positifs pour le bien-être, puis en assurant un appui financier suffisant (assorti d'un mandat) à la collecte de données nécessaire à l'évaluation rigoureuse et cohérente de ces interventions menées dans des conditions différentes (cf. Baker et al. 2010). L'étude GCS-REDD+ du CIFOR (Encadré 4) fournit un exemple du type de programme d'évaluation recherché qui vise à obtenir des résultats avec une validité interne et externe élevée par l'emploi de méthodes cohérentes et rigoureuses appliquées à toute une gamme de projets. Des porteurs de projet répartis en Afrique, en Asie et en Amérique latine ont accepté de collaborer à l'étude GCS-REDD+ pour contribuer à l'enrichissement des connaissances mondiales relatives à la REDD+. 


\subsection{Tirer les enseignements du passé}

La littérature qui évalue les réformes politiques concernant la gestion et la conservation des ressources naturelles nous livre des leçons importantes pour l'analyse des projets de REDD+. La première est que les méthodes d'évaluation d'impact rigoureuses quantifiant les changements qui se seraient produits en l'absence d'intervention ont rarement été appliquées aux investissements relatifs à la conservation (Ferraro et Pattanayak 2006, Schreckenberg et al. 2010). Par exemple, la plupart des évaluations publiées en matière de paiements pour services écosystémiques (PSE) sont des études de cas qualitatives, qui s'appuient sur la documentation produite par des organismes publics et des organisations non gouvernementales, des revues de la littérature grise, des entretiens avec des informateurs privilégiés et des évaluations rapides sur le terrain (Pattanayak et al. 2010). C'est également le cas des premiers projets de déforestation évitée (Caplow et al. sous presse).

Les initiatives d'application de méthodes rigoureuses d'évaluation d'impact aux interventions dans le domaine de la conservation sont en augmentation. Ainsi, le Fonds pour l'environnement mondial a financé un projet à concept expérimental pour analyser les impacts de PSE en Ouganda (FEM 2010). De même, la Wildlife Conservation Society a recueilli des données abondantes sur les conditions initiales dans les communautés situées à distance variable de nouvelles aires protégées au Gabon (Wilkie et al. 2006). Toutefois, dans le domaine de la conservation, l'évaluation d'impact est en général fondée sur la collecte de données ex post, relatives aux unités « traitées » et aux unités « témoins ou de contrôle ». Il peut s'agir par ex. de ménages ou de bassins hydrographiques situés à l'intérieur et à l'extérieur d'un site de projet de REDD+. Si l'effectif de l'échantillon est suffisamment important et que les données présentent une variabilité suffisante, différentes sortes d'analyses statistiques peuvent être possibles.

La procédure classique consiste à faire une régression des résultats par rapport à un indicateur de «traitement » de l'unité par le projet et tout facteur de confusion potentiel (estimation d'un modèle de régression du revenu des ménages en fonction de leurs caractéristiques et de leur appartenance ou non au projet de REDD+). Cependant, cette approche est critiquée parce quelle risque de trop dépendre de suppositions relatives à la distribution et de l'extrapolation à des unités traitées et non traitées très différentes. Les méthodes "d'appariement ", mises au point pour contrecarrer ces difficultés sont de plus en utilisées pour évaluer les résultats de politiques liées aux ressources naturelles et à la conservation. L'approche scientifique la plus récente consiste à combiner l'appariement et la régression pour obtenir des estimations d'impact deux fois plus solides.

La littérature récente consacrée à l'évaluation d'impact examine diverses interventions et leurs résultats et notamment l'impact causal :

- des quotas individuels transférables sur l'effondrement des pêches du monde entier (Costello et al. 2008) ;

- des moratoires sur le développement aux ÉtatsUnis (Bento et al. 2007) ;

- des aires protégées sur le couvert forestier au Costa Rica (Andam et al. 2008), à Sumatra (Gaveau et al. 2009a) et au plan mondial (Nelson et Chomitz 2009);

- des PSE sur le couvert forestier au Costa Rica (Arriagada 2008, Pfaff et al. 2008) ;

- de la gestion décentralisée sur le couvert forestier en Inde (Somanathan et al. 2009) ;

- de la décentralisation de la gestion des forêts sur le revenu des ménages tiré des forêts au Malawi (Jumbe et Angelsen 2006) et en Ouganda (Jagger 2008) ;

- des projets intégrés de conservation et développement sur les moyens d'existence des ménages (Weber et al. sous presse) ;

- des aires protégées sur la réduction de la pauvreté (Bandyopadhyay et Templo 2009, Andam et al. 2010, Sims 2010).

Quels que ce soient la méthode précise et le thème des évaluations d'impact, il y aurait tout intérêt à s'appuyer sur des données relatives aux conditions précédant le lancement de l'intervention : souvenirs des ménages, informations reconstituées au moyen de la télédétection ou de données secondaires ou, 
(de préférence), enquêtes préalables. Certaines études antérieures ont été confrontées à la difficulté d'éliminer les explications autres pour justifier les impacts observés en raison de l'insuffisance des données relatives à la période précédant l'intervention. Les données de l'avant projet permettent de comparer la variation des critères de jugement pour des échantillons appariés d'unités de contrôle et de traitement, ces unités pouvant être appariées en fonction de leurs caractéristiques avant quelles ne soient affectées par l'intervention : cette démarche permet de mettre en évidence les facteurs connus par des observations de terrain pour avoir une influence sur la sélection des sites et l'inclusion des participants dans le projet.

\subsection{Comment s'y prendre}

Le reste de ce guide s'articule comme suit : Dans la section suivante, nous discutons du rôle central du raisonnement contrefactuel dans l'évaluation des projets de REDD+, en présentant plusieurs méthodologies de recherche qui permettent de réaliser une évaluation ex post des impacts sur le bien-être économique et social. Nous étudions les conditions et facteurs déterminant le choix de la méthodologie de recherche, ainsi que certains des aspects techniques liés à la mise en œuvre de ces méthodologies. Dans la Section 3, nous préconisons l'élaboration de modèles de causalité et de théories du changement pour expliquer les raisons pour lesquelles l'intervention de REDD+ est supposée avoir une influence sur le bien-être économique, physique, psychologique des usagers de la forêt et des principaux acteurs. Nous soulignons l'utilité d'une méthodologique mixte, analysons les modalités de mise en œuvre des projets de REDD+ et fournissons une interprétation des conclusions des évaluations d'impact rigoureuses. Dans la Section 4, nous formulons des recommandations sur les facteurs pratiques à prendre en considération lorsqu'il s'agit de concevoir des évaluations rigoureuses de projets de REDD+ : budgets, capacités nécessaires et aspects liés à l'éthique. Cet exposé est accompagné d'une série de fiches techniques (Annexe A) dans lesquelles nous présentons la terminologie employée, le choix et la mesure des variables, ainsi que des informations plus techniques sur les méthodologies de recherche et les analyses de répartition possibles. Ce guide se penche donc sur les méthodologies de recherche pour l'évaluation d'impact ; les autres questions centrales dans ce domaine (telles que la mise au point et la mesure d'indicateurs) sont en effet bien décrites dans les autres ressources présentées en Annexe B. Tout au long de ce guide, nous nous servons de méthodes et d'éléments tirés de l'étude GCS-REDD+ du CIFOR (Encadré 4), car elle constitue un exemple de mise en œuvre d'un des plans expérimentaux les plus fiables présentés dans ce guide : le plan « avant-après/contrôleintervention » (BACI). L'Annexe $\mathrm{C}$ fournit les recommandations techniques et les questionnaires employés pour la collecte de données sur des sites de projets REDD+ en Afrique, en Asie et en Amérique latine.

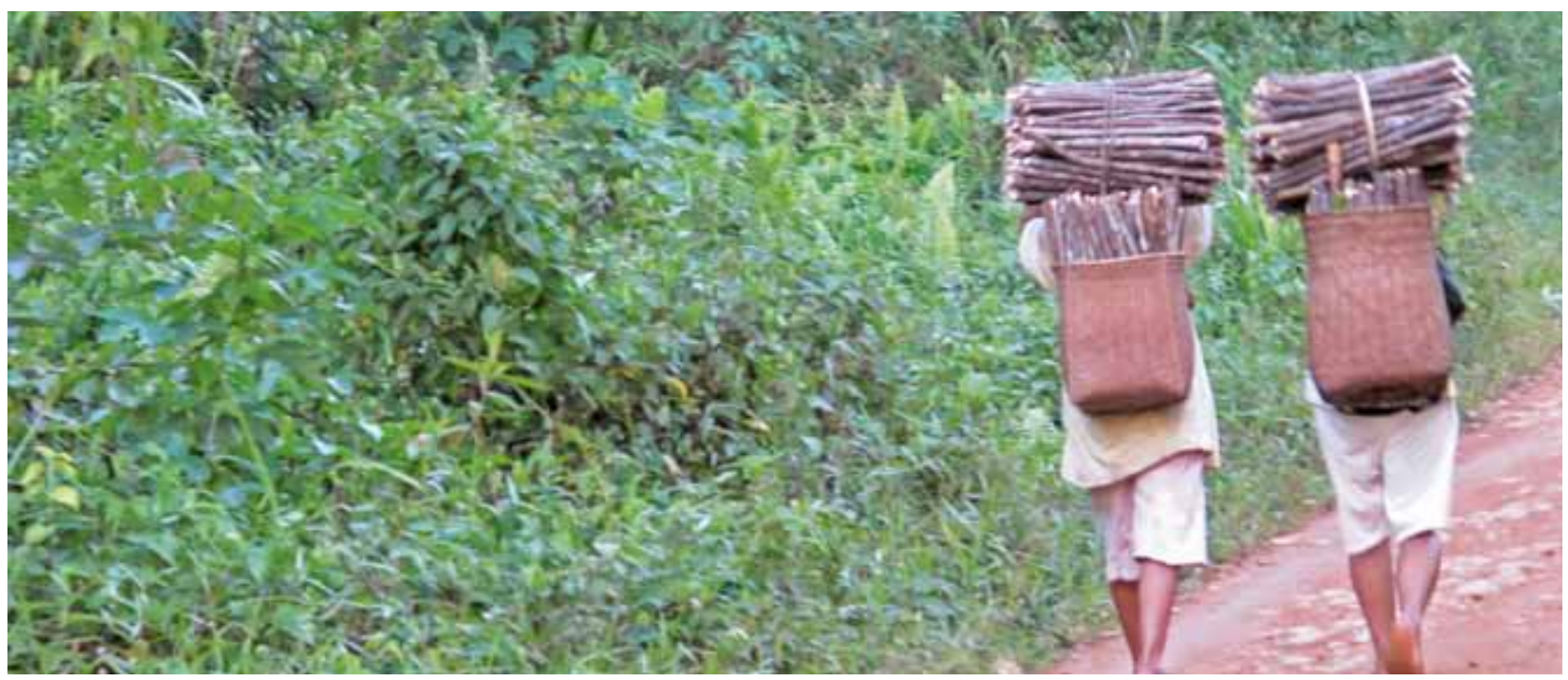

Deux habitants des forêts rentrent chez eux après le ramassage du bois de feu dans le district de Ketapang, à Kalimantan Ouest, Indonésie (@ Andini Desita Ekaputri/CIFOR) 


\section{Utiliser le raisonnement contrefactuel pour mieux connaître les projets de REDD+}

$\mathrm{L}$

e débat concernant la REDD+ est alimenté en partie par la diversité des lieux, suppositions, échelles et méthodes employées pour étudier ses répercussions sur le carbone, la biodiversité et les moyens de subsistance. Dans bien des cas, les divergences d'opinions sont dues aux suppositions très différentes qui sont faites à propos d'éventuelles solutions de rechange : à quoi ressemblerait la planète, ou une région donnée sans la REDD+? Ce manque d'homogénéité mais aussi de réflexion clairement articulée sur les scénarios contrefactuels contribuent à attiser la polémique et ne permettent guère d'analyser de manière systématique les caractéristiques et conditions associées aux arbitrages ou aux complémentarités. Même s'il n’est pas toujours possible ni souhaitable d'harmoniser les méthodes appliquées dans les différents projets, nous insistons sur la nécessité d'avoir des principes communs s'agissant d'évaluer les pilotes de REDD+ de première génération. L'un de ces principes est que toute évaluation doit établir et préciser la situation contrefactuelle correspondante. Dans cette section, nous étudions les différentes approches du raisonnement contrefactuel appliqué à la $\mathrm{MNV}$ du carbone et à l'évaluation d'impact. La fiche technique 1 compare les terminologies utilisées dans ces deux domaines.

L'importance de la surveillance et de l'évaluation (M\&E) est unanimement reconnue par tous les porteurs de projet dans le domaine de la conservation, du développement et a fortiori de la REDD+. La M\&E comprend la définition des résultats ou objectifs souhaités (augmentation de la richesse des ménages et de la préservation de la biodiversité par ex.) (Fiche technique 2) et de modèles de concept pour représenter les liens de causalité reliant le projet et les résultats souhaités (Fiche technique 8). Dans la REDD+, les méthodes de comptabilisation du carbone (ou MNV) ont été au cœur des préoccupations, et à juste titre puisqu'une comptabilité fausse remettrait en cause l'objectif fondamental de la réduction des émissions de gaz à effet de serre. Comme souvent en M\&E, les efforts les plus importants ont été consacrés à la définition et à la mesure des résultats, parmi lesquels figurent les émissions par hectare selon les catégories d'utilisations du sol, et les changements de surface en fonction de ces catégories (par ex. Global Observation for Forest and Land Cover Dynamics (GOFC/GOLD) 2009).

Il existe également une littérature abondante et de nombreuses propositions (Parker et al. 2009) et modèles différents (Hertel et al. 2009 par ex.) consacrés à la définition de scénarios contrefactuels d'utilisation du sol pour répondre au besoin de base de référence pour l'attribution de crédits de REDD+ dans un système de REDD+ international. Moins abondante est la littérature publiée (Brown et al. 2007 par ex.) qui traite de l'estimation des situations contrefactuelles d'utilisation du sol dans les projets ayant pour objectif de générer des crédits carbone (cf. Annexe B.5) ; les méthodes et recommandations pratiques qui l'accompagnent sont toutefois en plein développement. Ces méthodes portent sur les modalités de définition d'une situation contrefactuelle ex ante crédible (niveau de référence) et de fixation d'objectifs ex ante crédibles (c'est l'additionnalité, dont la nécessité est attestée 
par les résultats financiers insuffisants, les barrières institutionnelles et l'insuffisance d'adoption antérieure). L'évaluation ex post des résultats à l'intérieur de la zone du projet fait partie de la surveillance et de la vérification. Cependant, la plupart des méthodologies (dont le boisement/ reboisement dans le cadre du Mécanisme pour un développement propre (MDP) et les méthodes de REDD+ proposées par l'association VCS et le Registre américain du carbone (ACR)) n'exigent pas la surveillance ni la vérification continues dans des zones de contrôle ou de référence situées en dehors du projet.

Ces méthodologies exigent en général que les niveaux de référence (ou situations contrefactuelles) soient régulièrement réévalués et actualisés. Dans le cadre de cette réévaluation, les informations relatives aux émissions réelles dans des zones de référence deviennent pertinentes pour l'établissement d'une nouvelle situation contrefactuelle ex ante. L'Alliance CCBA exige également l'analyse des impacts ex ante d'un projet sur les moyens d'existence et la biodiversité par rapport à la situation contrefactuelle, ainsi que la vérification périodique de ces estimations. L'évaluation ex post quantitative et empirique que nous préconisons dans ce guide est très semblable à la démarche de vérification de projet comprenant la réévaluation (la révision) du niveau de référence.

La différence principale entre les méthodes présentées dans ce guide et la MNV du carbone provient du fait que les porteurs de projet se concentrent généralement sur l'élaboration de scénarios de niveaux de référence ex ante définissant les crédits pouvant être attendus si les résultats prévus se réalisent dans la zone du projet, tandis que les méthodes présentées ici s'attachent à s'approcher le plus possible de ce qui se serait passé dans la situation contrefactuelle en partant d'informations ex post. L'autre différence entre la comptabilité carbone et l'évaluation des impacts sur les moyens de subsistance des populations concerne la mesure des résultats, opération plus ou moins délicate. Dans le cas du carbone, la reconstitution d'un historique de données relatives à la couverture terrestre est généralement possible grâce à la télédétection et peut servir de base tant aux projections ex ante du niveau de référence du carbone et quà l'analyse des émissions de carbone ex post dans la zone du projet. En revanche, les données de source secondaires concernant les conditions socioéconomiques sont généralement beaucoup plus limitées, ce qui veut dire que les porteurs de projet ou les chercheurs doivent recueillir les données primaires eux-mêmes en commençant avant le lancement du projet. Comme nous l'indiquons dans la Fiche technique 6, ces données peuvent être obtenues en faisant appel aux souvenirs des personnes interrogées concernant les conditions antérieures au projet, mais cette approche a ses limites.

La comptabilité carbone d'un projet et la recherche design (design-based research) dans le domaine des impacts sociaux, dont il est question ici, sont apparentées sur certains grands points. Ainsi, ces deux domaines de travail :

1. reposent sur une estimation crédible de la situation contrefactuelle (appelée « niveau de référence » dans la littérature consacrée au carbone);

2. sont axés sur la mesure fiable de critères de jugement pertinents (changements d'utilisation du sol et émissions par rapport au revenu, à la consommation et au niveau de richesse) ;

3. cherchent à déterminer si les résultats apparents sont imputables au projet ( appelés « tests d'additionalité » dans les référentiels du marché du carbone volontaire, et « effets du traitement » en évaluation d'impact) ;

4. se préoccupent des retombées et des fuites (en définissant par ex. un périmètre de fuites qui sera contrôlé dans le cas des référentiels du marché volontaire du carbone, et en vérifiant la «SUTVA» ou l'hypothèse d'absence d'effets de diffusion du traitement dans le cas des évaluations d'impact).

En pratique, si un projet conduit à une rétention de carbone (additionnalité), c'est qu'il aura sûrement influé sur le comportement et le bienêtre humains, ce qui aura probablement créé des fuites, les hommes réagissant toujours en tentant de maximiser l'utilité lorsqu'ils se trouvent confrontés à des contraintes. Si l'additionnalité dans la zone du projet a été obtenue au détriment du bien-être économique (par réduction de la 
production par ex.), des fuites vers d'autres zones ont toutes les chances de se produire ; lesquelles se traduiront par une augmentation du bien-être (par augmentation de la production par ex.). Cette constatation concorde bien avec l'inquiétude exprimée fréquemment selon laquelle les fuites sont susceptibles de conduire à plus d'émissions de carbone (c'est-à-dire qu'elles s'inscrivent négativement au bilan du carbone). Ainsi, la vraie question en matière d'impacts socioéconomiques de la REDD+ est peut-être celle de la répartition de ces impacts plus que celle du total net des impacts sur le bien-être. Cette question est abordée dans la Fiche technique 9 qui traite des impacts sur la répartition du bien-être, et plus loin dans cette section, lorsque nous définissons les zones et usagers de la forêt à « traiter » et ceux qui serviront de témoins. Même si les impacts de projet peuvent s'étendre à un périmètre beaucoup plus important, il reste essentiel d'étudier les impacts socioéconomiques « locaux" pour un certain nombre de raisons, liées aux droits et revendications locales relatives aux ressources forestières, au rôle direct des acteurs locaux dans la détermination de l'avenir de la forêt (et par conséquent la permanence des crédits de carbone) et la condition relativement défavorisée de ces acteurs locaux, méritant à ce titre une attention spéciale.

\subsection{Choix de la méthodologie de recherche : hypothèses fondamentales}

Dans ce guide, nous décrivons une série de méthodes d'évaluation d'impact pour les projets de REDD+ qui sont des activités infranationales mises en œuvre dans une zone géographique définie et/ou faisant intervenir un sous-ensemble d' "usagers de la forêt ", comprenant des ménages mais aussi éventuellement des entreprises, qui sont propriétaires de la forêt, la gèrent ou en utilisent les ressources. Les méthodes décrites peuvent également servir à estimer les impacts dans le cadre de la participation à des programmes nationaux (par ex. les PSE au Costa Rica et au Mexique), si toutefois il y a bien une variation au niveau de la couverture du programme, c'est-à-dire si l'on dispose de données sur des participants et des non participants (si tous les usagers de la forêt qui sont éligibles ne participent pas par ex.).
Ces projets et programmes se caractérisent par le fait que l'impact global dépend de la proportion de population concernée (régions retenues, catégories d'usagers de la forêt choisies pour participer par ex.) et de l'effet de causalité de l'intervention sur cette population. Les méthodes présentées dans ce guide visent principalement à estimer l'effet de causalité sur la population concernée. En évaluation d'impact, cet effet est appelé l'effet $\mathrm{du}$ «traitement». Comme nous le verrons plus loin dans cette section, un " traitement " peut correspondre à des interventions différentes, allant du propriétaire forestier pouvant se lier par un contrat PSE de sa propre initiative, aux répercussions des changements de gestion dans une concession forestière sur les populations dépendantes des forêts situées à proximité.

Les méthodes d'évaluation d'impact présentées dans ce guide se fondent sur l'analyse statistique de preuves empiriques, à savoir d'observations (obtenues par enquête, télédétection ou données secondaires) de résultats dans le monde réel. Ces méthodes sont appliquées dans les conditions de traitement suivantes :

1. Il faut des régions ou des usagers de la forêt recevant le traitement, et d'autres qui ne le reçoivent pas. Le corollaire de cette condition est qu'il faut pouvoir imaginer qu'une région ou des usagers de la forêt traités puissent ne pas avoir reçu le traitement. Ainsi, ces méthodes ne peuvent être appliquées dans le cas d'une politique nationale affectant toute la population d'un pays ou d'une certaine catégorie, comme une loi concernant tous les groupes autochtones.

2. Les participants doivent être nombreux pour que l'estimation de l'effet moyen du traitement ait un sens (il pourra s'agir de l'effet moyen sur les traités ou sur toute la population). Par exemple, ces méthodes ne peuvent pas convenir pour estimer les impacts sur une seule grande entreprise d'exploitation forestière dans un pays où quelques entreprises de cette nature uniquement exerceraient cette activité.

Lorsque des usagers de la forêt en nombre suffisant sont affectés par un projet sans pour autant recevoir le traitement dans leur totalité, les méthodes 
présentées dans ce guide peuvent être employées pour évaluer les impacts suivants :

1. Evaluation ex post d'impacts réalisés, donc des impacts que le projet a causés. Cet exercice se rapproche fortement du processus de vérification mené dans l'optique des normes actuelles du marché volontaire du carbone, consistant à réévaluer et à actualiser le niveau de référence du projet. Il diffère des projections ex ante exigées dans le cadre de la validation de projet par rapport aux référentiels de ce marché. La plupart des méthodes présentées dans ce guide diffèrent également de la vérification de ce qui s'est produit dans la zone du projet par rapport au niveau de référence projeté, souvent exigée pour la vérification initiale de projet. Juger d'un projet par rapport à un niveau de référence projeté réduit l'incertitude relative aux crédits d'émission de carbone attendus, puisque ces crédits ne dépendraient alors que des résultats du projet comparativement au niveau de référence projeté, et non de tous les facteurs indépendants du projet, susceptibles d'avoir une influence sur la situation contrefactuelle. En mesurant les impacts d'un projet par rapport à une situation contrefactuelle représentative de ce qui se serait passé si le projet n'avait pas eu lieu, les méthodes présentées dans ce guide n'apportent pas tellement la certitude souhaitée par les investisseurs. Elles apportent plutôt des informations favorisant la capitalisation de l'expérience pour l'avenir, plus que le jugement des performances passées d'un projet.

2. Evaluation des impacts attendus dans un créneau temporel pertinent pour la formulation de politiques. Pour que l'évaluation d'impact permette d'éclairer les débats politiques sur la REDD+, les résultats d'évaluation doivent être disponibles en temps utile. Or, ce délai est trop court pour permettre l'observation empirique des impacts à long terme de la REDD+, en supposant que les décisions relatives aux stratégies d'atténuation des changements climatiques seront adoptées avant que les impacts à long terme ne soient constatés (à l'horizon de 10 ans). Cependant, comme l'explique la Fiche technique 8, les résultats observés pourraient être les étapes intermédiaires d'un modèle de causalité rendant compte du long terme. Ainsi, des changements affectant la détention des actifs ou les fluctuations des migrations saisonnières qui peuvent être observés pourraient devenir des variables essentielles pour la modélisation de l'augmentation du bien-être et de l'évolution de l'utilisation du sol à long terme. Il est certain que les impacts des projets de REDD+ à longue échéance ont une importance capitale pour l'atténuation des changements climatiques. Nous pouvons avoir plus confiance en des projections à long terme qui reposent sur des hypothèses concordant avec les conclusions d'évaluations empiriques ex post d'impacts intermédiaires, auxquelles on a abouti en appliquant des méthodes rigoureuses ayant permis d'éliminer les explications contradictoires.

3. Evaluation des impacts directs du traitement. Tout au long du guide, nous avons effectué la simplification consistant à considérer que le traitement est binaire : un usager de la forêt est soit « traité », soit «non traité " par un projet. Cela ne veut pas nécessairement dire qu'il habite à l'intérieur des limites du projet, comme le veut la définition dans le cadre de la comptabilisation du carbone. Par exemple, le traitement pourrait se baser sur la résidence ( $\mathrm{au}$ début du projet) dans la zone élargie du projet qui comprend les communautés adjacentes, comme c'est le cas dans les référentiels CCB. Il est évident cependant que la réalité est beaucoup plus nuancée, la plupart des projets de REDD+ ayant des dimensions et des niveaux de participation multiples, comme des répercussions directes et indirectes sur les ménages et les entreprises. Nous étudions ciaprès les difficultés posées par la définition de la participation, ou traitement, ainsi que les différentes variantes possibles.

Le Tableau 1 peut aider à choisir une méthodologie de recherche permettant de mesurer l'impact des projets de REDD+, si les conditions décrites cidessus sont respectées. Si elles le sont, les méthodes proposées peuvent servir à analyser l'impact de tout type d'intervention de projet de REDD+ en fonction de critères de jugement mesurables, comme le couvert forestier, la biodiversité et le 
Tableau 1. Possibilités de méthodologies de recherche ayant pour objectif l'évaluation d'impact ex post fondée sur l'évidence empirique

\begin{tabular}{|c|c|c|c|c|c|}
\hline $\begin{array}{l}\text { Commencement } \\
\text { avant le } \\
\text { lancement du } \\
\text { projet? }\end{array}$ & $\begin{array}{l}\text { Intérêt suscité/ } \\
\text { budget pour } \\
\text { la collecte de } \\
\text { données de } \\
\text { contrôle? }\end{array}$ & $\begin{array}{l}\text { Capacité } \\
\text { d'influer sur } \\
\text { la conception } \\
\text { du projet? }\end{array}$ & $\begin{array}{l}\text { Méthodologie de } \\
\text { recherche }\end{array}$ & $\begin{array}{l}\text { Élaboration } \\
\text { de scénarios } \\
\text { contrefactuels en... }\end{array}$ & $\begin{array}{l}\text { Nécessité } \\
\text { d'utiliser des } \\
\text { techniques } \\
\text { d'appariement? }\end{array}$ \\
\hline Oui & Oui & Oui & $\begin{array}{l}\text { Randomisation } \\
\text { (Fiche } \\
\text { technique 3) }\end{array}$ & $\begin{array}{l}\text { Allocation aléatoire } \\
\text { des sites de projet et } \\
\text { de contrôle }\end{array}$ & Peut-être \\
\hline Oui & Oui & Non & $\begin{array}{l}\text { Avant-après/ } \\
\text { contrôle- } \\
\text { intervention } \\
\text { (BACl) (Fiche } \\
\text { technique 4) }\end{array}$ & $\begin{array}{l}\text { Données } \\
\text { d'observation aux } \\
\text { sites de contrôle } \\
\text { avant et après } \\
\text { l'intervention }\end{array}$ & Oui \\
\hline Oui & Non & Non & $\begin{array}{l}\text { Avant-après (BA) } \\
+ \text { projection } \\
\text { d'un scénario } \\
\text { contrefactuel } \\
\text { (Fiche } \\
\text { technique 5) }\end{array}$ & $\begin{array}{l}\text { Modèles, souvent } \\
\text { construits à partir } \\
\text { de tendances } \\
\text { historiques }^{\mathrm{a}}\end{array}$ & Peut-être \\
\hline Non & Oui & Non & $\begin{array}{l}\text { appariement } \\
\text { des unités } \\
\text { de contrôle- } \\
\text { intervention }(\mathrm{Cl}) \\
\text { (Fiches techniques } \\
5 \text { et } 7 \text { ) }\end{array}$ & $\begin{array}{l}\text { Données } \\
\text { d'observation (et } \\
\text { aussi souvent recueil } \\
\text { de souvenirs) aux } \\
\text { sites de contrôle } \\
\text { après l'intervention }\end{array}$ & Oui \\
\hline Non & Non & Non & $\begin{array}{l}\text { Autoréférentielle } \\
\text { ou rétrospective } \\
\text { (Fiche } \\
\text { technique 6) }\end{array}$ & $\begin{array}{l}\text { Estimation des } \\
\text { « changements dus } \\
\text { au projet » à partir } \\
\text { d'opinions et/ou de } \\
\text { souvenirs }\end{array}$ & Non \\
\hline
\end{tabular}

a Les données $d^{\prime}$ " "avant-après » relatives au projet peuvent également être combinées à des modèles de simulation de scénarios contrefactuels, ou à des analyses qualitatives de situations contrefactuelles réalisées en récoltant les points de vue des populations. Cette approche présente de grandes similitudes avec l'élaboration de scénarios contrefactuels de déforestation/ dégradation dans le cadre des projets de REDD+ : consulter la Fiche technique 8.

bien-être économique et social. Dans le cas de la dimension bien-être, les critères de jugement et indicateurs possibles sont nombreux. Bien entendu, le choix des indicateurs doit découler d'une décision bien pensée (cf. Fiche technique 2), mais il ne dépend pratiquement pas du plan expérimental choisi, sauf dans le cas de la méthodologie dans laquelle les participants doivent se souvenir des conditions antérieures au projet (ou imaginer ce que seraient les conditions sans projet). Dans ce cas alors, l'évaluation se limitera aux types de critères et au niveau de précision des souvenirs que l'on peut raisonnablement espérer de la part des personnes interrogées (cf. Fiche technique 6).

\subsection{Choix d'une méthodologie de recherche : questions fondamentales}

Cinq plans expérimentaux d'évaluation des impacts d'un projet de REDD+ sont présentés dans le Tableau 1. Le choix du plan est conditionné par les réponses apportées à trois questions (dans les colonnes de gauche du Tableau 1), détaillées ci-après. Ce tableau et ces questions ont été formulées dans l'optique de la question d'évaluation la plus simple possible, à savoir : quel est l'impact du projet ? (À la section 3 , nous reparlerons de l'évaluation de l'impact de la mise en œuvre d'un projet) 
Q1. La conception de l'évaluation d'impact précède-t-elle l'intervention de REDD+ à évaluer? Le plus souvent, les évaluations d'impact sont lancées après la conception et la mise en œuvre de l'intervention, toutes les ressources dont on dispose au début du projet étant consacrées à la mise en œuvre, c'est-à-dire à la mise en place de l'intervention. Cependant, les estimations ex post des impacts de projet seront plus crédibles si un concept expérimental est intégré au projet et/ ou si des données sont collectées ex ante, avant que l'intervention ne commence. Nous étudierons plus avant dans cette section la question de savoir comment définir le point de départ du projet.

\section{Q2. L'évaluation bénéficie-elle de ressources et} d'une mobilisation suffisantes pour développer la collecte d'informations relatives aux usagers de la forêt qui ne sont pas inclus dans le projet ? Il s'agit d'une tâche nécessitant un investissement allant au-delà des exigences des référentiels des marchés volontaires du carbone (VCS ou CCBA par ex.). La définition d'un niveau de référence pour la validation demande en général une analyse des événements se produisant dans la région dans son ensemble, mais non la collecte d'informations continue en dehors de la zone du projet. De la même façon, la surveillance et la vérification concernent la zone du projet, et le cas échéant, un «périmètre de fuite " ou zone tampon. Le renouvellement de la période prise en compte pour l'attribution de crédits de REDD+ exige en général l'analyse des changements d'utilisation du sol dans la région de référence (située au-delà de la zone du projet) dans le but de définir un autre niveau de référence pour la période de comptabilisation suivante. Cette analyse s'appuie souvent sur la télédétection accompagnée d'informations recueillies auprès des usagers de la forêt au moyen de méthodes participatives ou qualitatives. La collecte de données sur les usagers de la forêt en dehors de la zone du projet au moyen d'enquêtes nest pas une pratique courante dans les projets de REDD+, ni dans les initiatives de conservation (cf. Wilkie et al. 2006 qui présentent une exception).

Q3. Si la conception de l'évaluation est effectuée avant la mise en ouvre et qu'il y a suffisamment de ressources pour collecter des données sur les non participants, la troisième question à se poser est la suivante : l'intervention elle-même peutelle être conçue pour faciliter l'évaluation ? Plus précisément, les évaluateurs peuvent-ils avoir une influence sur le choix des personnes ou des zones qui recevront l'intervention et sur le moment de cette intervention? La réponse à cette question dépendra à la fois de la relation entre l'évaluateur et le porteur de projet et de la nature de l'intervention à évaluer.

Elle dépend également de son échelle : les évaluateurs ne pourront probablement pas (et ne souhaiteront sans doute pas) influer sur le choix de l'état ou de la province où sera mis en œuvre un projet; en revanche, à l'autre extrême au regard de l'échelle, les PSE constituent des échanges fondés sur le volontariat : à ce titre, la participation de chaque ménage ou de chaque exploitation agricole ne doit pas reposer sur la contrainte, mais la localisation initiale du programme de PSE (villages particuliers par ex.) pourrait être choisie de manière à faciliter l'évaluation ex post de l'impact de ce programme.

\subsection{Choix d'une méthodologie de recherche : options fondamentales}

Il est aussi possible de répondre aux trois questions du Tableau 1 en commençant par la dernière, comme le montre la Figure 2. Cette démarche met en évidence la décision essentielle de l'intégration d'un concept expérimental dans l'intervention. Pour utiliser un tel concept pour mesurer l'impact d'un projet dans sa globalité, la conception de l'évaluation doit être réalisée avant l'intervention, et de plus, il faut des ressources pour collecter les données sur les témoins des groupes de contrôle. Si ces conditions sont réalisées, sur la partie supérieure du schéma de la Figure 2, les réponses aux questions Q1, Q2 et Q3 seront toutes « oui ». Dans un tel cas, le traitement peut être randomisé (allocation aléatoire comme dans un tirage au sort), ce qui permet d'assurer qu'en moyenne, les usagers de la forêt inclus dans le projet seront semblables aux usagers non inclus du point de vue de leurs caractéristiques observables, telles que la taille de leur famille, mais aussi de caractéristiques non observables, telles que leur préférence pour le travail en forêt. Comme ni les usagers de la forêt, ni les administrateurs d'un programme 


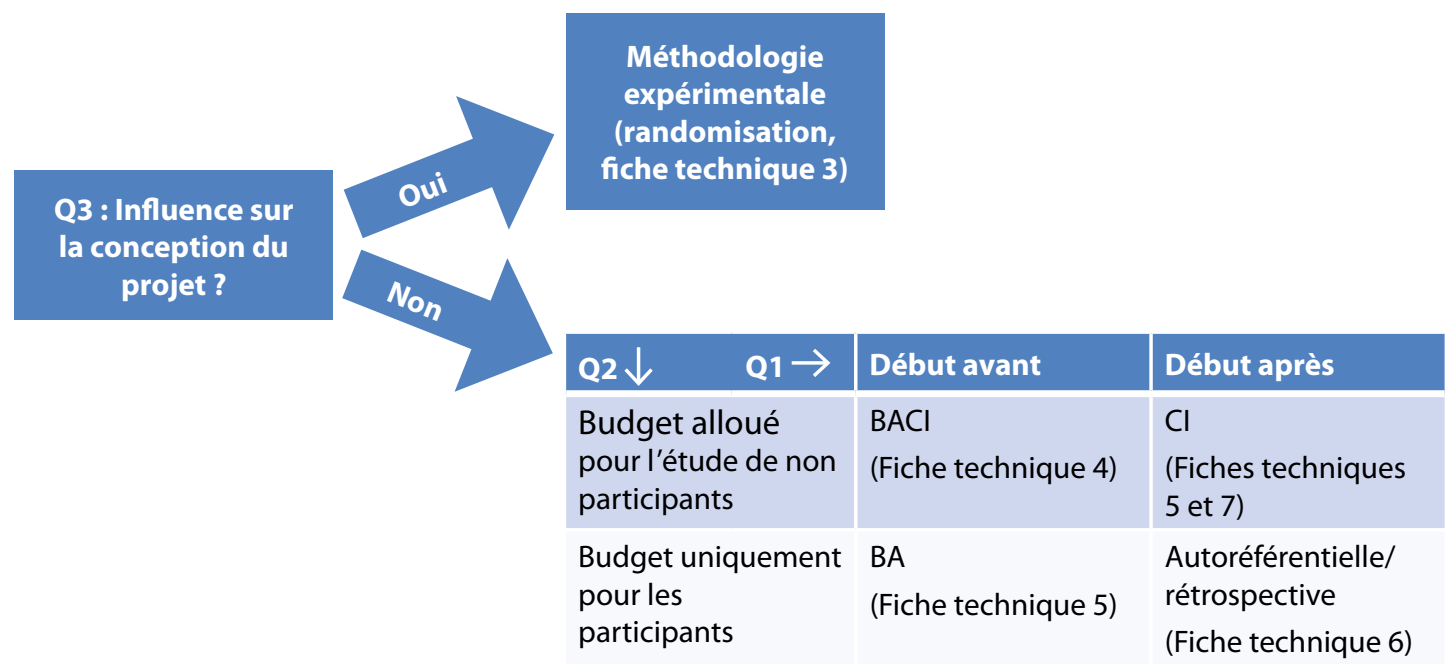

Figure 2. Arbre décisionnel pour le choix d'une méthodologie de recherche

ne décident qui sera inclus dans le projet, cette décision résultant d'un procédé de randomisation indépendant, il n'existera aucun lien entre le traitement et d'autres facteurs susceptibles d'influer sur les résultats. Il n'y aura donc pas de biais de sélection (cf. Fiche technique 3). Cette procédure fournit les estimations d'impact causal les plus crédibles. Dans d'autres domaines, elle permet d'élargir et de diffuser des modèles politiques prouvés efficaces (on attribue par ex. à l'évaluation d'impact rigoureuse du programme de transfert monétaire conditionnel au Mexique la mise en place de programmes équivalents dans d'autres pays (Banque mondiale 2009).

La randomisation peut s'effectuer de diverses manières. Par exemple, lorsqu'un projet est sursouscrit (lorsque le nombre de ménages qui souhaitent des contrats de PSE ou des emplois dans le cadre du projet dépasse les fonds disponibles), les participants pourraient être sélectionnés de manière aléatoire dans un pool de candidats. Dans le cas d'un lancement de projet par étapes, les premières zones de mise en œuvre du projet pourraient être choisies aléatoirement. La possibilité de participer à un contrat de PSE pourrait d'abord être donnée aux usagers de la forêt dans un groupe de villages choisis de manière aléatoire, puis, trois années après, à un autre groupe sélectionné aussi de manière aléatoire.
Pour tirer des conclusions sur les impacts causaux, des données doivent être collectées sur les usagers de la forêt inclus (à savoir les traités) et ceux non inclus dans le projet (à savoir les témoins), l'inclusion ou la non inclusion étant déterminée de manière aléatoire. Si le projet permet aux usagers de la forêt d'en tirer des bénéfices (comme dans le cas des PSE), il pourrait être opportun pour des raisons politiques ou éthiques de proposer aux usagers du groupe de contrôle de participer au projet à une date ultérieure. Ce principe cadre bien avec les contraintes financières et les plans d'intensification de la mise en œuvre qui sont souvent associés aux projets, mais il est plus difficile à appliquer dans les cas où le financement n'est garanti que pour une courte durée.

Les données relatives aux usagers de la forêt inclus et non inclus doivent être recueillies de préférence avant et après la mise en œuvre du projet, de manière à vérifier qu'ils présentent une distribution initiale de caractéristiques équivalente (à effectuer avant le projet) et à comparer les résultats obtenus (à effectuer après le projet). (Il faut au moins que les données relatives aux caractéristiques et résultats du traitement soient collectées après l'intervention.) Si l'on s'attend à ce que les échantillons du groupe d'usagers de la forêt traités et du groupe de contrôle soient similaires du fait de la randomisation, chaque opération d'allocation aléatoire peut produire 
des échantillons présentant des différences de caractéristiques initiales significatives sur le plan statistique. Dans ce cas, les méthodes d'ajustement, présentées ci-après, peuvent être utilisées pour définir et attribuer des coefficients de pondération à des sous-échantillons définis dans le groupe de contrôle.

Même si la Figure 2 présente deux ramifications principales, il existe une troisième éventualité, qui se produit lorsque les porteurs de projet intègrent de manière fortuite un élément aléatoire dans le choix des usagers de la forêt inclus dans le projet, créant ainsi « une expérience dans les conditions naturelles ». Prenons un exemple dans lequel des sites de projet sont choisis en fonction de l'emplacement de certaines parcelles correspondant à un ancien inventaire botanique. Si la position de ces parcelles ne correspond plus aux conditions biophysiques et socioéconomiques actuelles, elle pourrait être utilisée comme «variable instrumentale » de la participation au projet. Pour que cette approche soit utile pour l'évaluation, il faut que l'élément aléatoire soit un déterminant puissant d'inclusion dans le projet mais qu'il ne présente aucun lien avec les résultats. Ce type d'élément aléatoire apparaît rarement dans les projets, mais quand il est présent, il est particulièrement intéressant de réaliser une approximation du concept expérimental via la méthode des variables expérimentales (Angrist et Pischke 2009) en recueillant des données sur cet élément.

Dans le cas où l'on ne pourrait mettre en place un concept expérimental (ou une « expérience dans les conditions naturelles »), la Figure 2 conduit à une matrice de méthodologies de recherche « quasi-expérimentales». La méthodologie la plus couramment employée par les porteurs de projet est BA, qui compare les résultats dans la zone du projet « avant » et " après » l'intervention (souvent complétée par la modélisation de résultats contrefactuels). En revanche, la méthodologie la plus fréquemment évoquée dans la littérature scientifique traitant de l'évaluation d'impact ex post est CI, qui compare les résultats dans les zones de contrôle et d'intervention. La méthodologie quasiexpérimentale qui donne les estimations d'impact les plus crédibles, mais dont la mise en œuvre est aussi la plus délicate et la plus coûteuse, est BACI ou " avant-après/contrôle-intervention ». Enfin, si on ne dispose d'aucune information antérieure au projet et si la collecte de données auprès de non participants au cours du projet n'est pas possible (en raison de contraintes budgétaires ou autres), il reste la solution consistant à demander aux participants d'évaluer eux-mêmes l'impact du projet ou de se souvenir des conditions antérieures à celui-ci. Même si le recueil des points de vue et des idées des participants à l'aide d'enquêtes doit faire partie de toute évaluation globale sérieuse (volet qualitatif ou participatif dans une approche méthodologique mixte par ex.), les données ainsi recueillies ne peuvent constituer des preuves "d'observation " substantielles. Par conséquent, cette solution est à considérer en dernière extrémité.

Si l'évaluation est lancée avant l'intervention (à savoir si la réponse à la question Q1 est « oui »), les données initiales collectées doivent porter sur:

1. les modalités d'inclusion des usagers de la forêt dans le projet, souvent appelées "procédé d'inclusion" (y compris des informations relatives aux candidats refusés, le cas échéant);

2. la valeur initiale des critères de jugement utilisés, tels que revenu, patrimoine, et dépendance à la forêt ;

3. les caractéristiques relatives aux ménages, exploitations agricoles ou villages ayant une influence sur (1) et (2).

Ces informations doivent être recueillies au moins pour les usagers de la forêt inclus dans le projet, afin de pouvoir utiliser la méthode BA (Fiche technique 5). Cette opération devrait s'intégrer sans trop de difficultés à la collecte de données initiales nécessaires pour concevoir un projet et obtenir la validation de celui-ci dans le cadre du marché volontaire du carbone. Afin de pouvoir mettre en œuvre les plans expérimentaux BA ou BACI dans leur intégralité, les données doivent être enregistrées de telle façon que les mêmes villages et ménages puissent être revisités dans la phase d' " après " (les informations doivent concerner des usagers de la forêt précis et non seulement des moyennes, de manière à pouvoir constituer une série de données de panel). En cas d'impossibilité, les méthodes d'ajustement (décrites ci-après) 
permettent de définir des cohortes de ménages semblables dans les phases de l'avant et de l'après projet (Shadish et al. 2002).

Même si le plan expérimental BA s'intègre généralement bien aux plans de collecte de données des porteurs de projet, il ne permet pas d'analyser clairement les impacts causaux du projet. Pour surmonter ce problème, on peut supposer que les conditions seraient restées constantes si le projet n’avait pas eu lieu, mais la réalité sera sûrement différente. On peut également faire une extrapolation à partir de tendances observées dans la période antérieure au projet (par ex. si les personnes interrogées rapportent une diminution des emplois et des revenus, on peut supposer que cette diminution se serait poursuivie). Cependant, les résultats obtenus pour les usagers de la forêt " traités " par le projet ont toutes les chances de dépendre non seulement des tendances antérieures et du projet, mais aussi de changements qui se produisent au même moment (concomitants). Les variations macroéconomiques, des changements politiques et réglementaires, des événements climatiques exceptionnels et des programmes et politiques n'ayant rien à voir avec les projets sont autant de facteurs susceptibles d'influer sur les résultats, en occultant ou exacerbant l'effet de l'intervention si les conditions antérieures au projet et les tendances passées sont prises comme "situation contrefactuelle contrefaite " (cf. Encadré 5 qui donne la définition de cette expression et des exemples). Dans les méthodologies élaborées pour le marché volontaire du carbone, on surmonte généralement ce problème en faisant une projection du résultat contrefactuel à l'aide d'un modèle (cf. Fiche technique 5).

La neutralisation empirique des effets éventuels de changements concomitants (par l'observation plutôt que par la modélisation) nécessite de récolter des données sur les non participants, c'est-à-dire les témoins du groupe de contrôle, ce qui signifie que la réponse à la question Q2 doit être « oui ». Pour estimer l'effet du traitement, il faut que les témoins soient semblables aux usagers de la forêt du projet au regard de toutes leurs caractéristiques susceptibles d'avoir une influence sur l'inclusion dans le projet, ainsi que sur les résultats de celui-ci (cf. Fiche technique 10 consacrée aux variables).
Il s'agit d'une condition nécessaire pour les deux méthodologies CI et BACI. Or, tout village, tout ménage possède des caractéristiques qui lui sont propres ; dans la réalité, les usagers " témoins » ne peuvent donc ressembler parfaitement aux usagers du projet. C'est également valable pour la randomisation dans laquelle les non participants du groupe de contrôle et les participants au projet sont sélectionnés de manière aléatoire. Néanmoins, en toute probabilité, et en moyenne, les participants des deux groupes présenteront des similitudes, puisqu'ils proviennent de la même population dans laquelle ils ont été choisis de manière aléatoire. Les méthodes quasi-expérimentales cherchent à reproduire ce protocole en sélectionnant un groupe de témoins, qui en toute probabilité et en moyenne, ressemblera au groupe traité : on dit alors que les échantillons de traités et de témoins sont " équilibrés ».

L'appariement, présenté dans la Fiche technique 7, s'utilise souvent pour définir des échantillons équilibrés de traités et de témoins. Avant la collecte de données, on procédera éventuellement à un pré-appariement en fonction de caractéristiques facilement observables : l'échantillon ainsi défini pourrait ainsi comprendre des témoins potentiels qui habitent dans la même zone écologique ou le même bassin versant que les usagers de la forêt traités dans le cadre du projet. Ce processus de pré-appariement pourrait également être réalisé en fonction de critères secondaires plus complexes. Par exemple, les villages témoins les plus semblables aux villages traités pourraient être sélectionnés par appariement statistique : on obtient ainsi un jeu de villages traités et témoins " appariés ", parmi lequel on sélectionne aléatoirement les usagers de la forêt dans la dernière étape d'échantillonnage. Des programmes économétriques comme STATA et $\mathrm{R}$ sont couramment utilisés : ils proposent en effet des progiciels ou des modules d'automatisation de l'appariement et produisent notamment des indicateurs de répartition " équilibrée » des caractéristiques entre les deux échantillons. Il est surtout important d'obtenir une répartition équilibrée des caractéristiques qui sont potentiellement des facteurs de confusion, c'est-àdire des facteurs qui conditionnent l'inclusion dans le projet et qui influent sur les critères de jugement utilisés pour évaluer les résultats. 


\section{Encadré 5. Le problème du raisonnement contrefactuel contrefait}

La simple comparaison des conditions postérieures au projet avec les conditions antérieures ou celles d'un autre site, suivie de l'imputation au projet de toute variation observée des critères de jugement, ne permet pas d'éliminer les explications contradictoires sur le plan du bien-être économique. C'est pourquoi ces comparaisons avant-après et avec-sans sont souvent appelées « raisonnements contrefactuels contrefaits ». Ces démarches inadaptées ont conduit à l'élaboration des approches d'évaluation d'impact étudiées dans ce guide dont la randomisation et la méthodologie de recherche BACI (Khandker et al. 2010). Des comparaisons simples avantaprès et avec-sans ont souvent été utilisées pour estimer les impacts des interventions dans le domaine de la conservation. Parmi les exemples les plus caractéristiques figurent Bruner et al. (2001) et Oliveira et al. (2007) qui s'appuient sur des comparaisons avec-sans pour estimer l'impact des aires protégées sur la biodiversité et le recul de la forêt, respectivement. Les exemples suivants illustrent comment ces « analyses contrefactuelles contrefaites » peuvent conduire à attribuer à un projet de REDD+ des résultats erronés sur le plan du bien-être.

Imaginons qu'un projet de REDD+ a été lancé dans une région boisée située non loin de la capitale dans un pays $X$. Des données détaillées relatives au bien-être économique sont recueillies parmi les populations dans la zone du projet, juste avant que ne débutent les activités, puis de nouveau cinq années plus tard (cf. Figure 3). Les données collectées mettent en évidence une dégradation moyenne considérable du bien-être économique. L'évaluation du projet attribue cette dégradation du bien-être au projet de REDD+, à la grande déception des porteurs du projet, des bailleurs de fonds et du gouvernement du pays $X$. Or, il se trouve qu'au moment où débutait le projet, le pays $X$ dévaluait sa monnaie et la fonction publique subissait des baisses importantes d'effectifs et de salaires, entraînant une période de déclin du bien-être économique de plusieurs années. Les catégories de la population fortement dépendantes des revenus de la fonction publique et ayant de nombreux échanges commerciaux dans l'économie de marché ont été les plus touchées. Même si le site du projet de REDD+ n'a pas été épargné par cette dégradation du bien-être économique, les recettes tirées du carbone ont en fait permis aux populations de mieux conserver leur niveau de vie qu'elles n'auraient pu le faire en l'absence du projet de REDD+. Cependant la comparaison simple avant-après est passée à côté de cet impact.

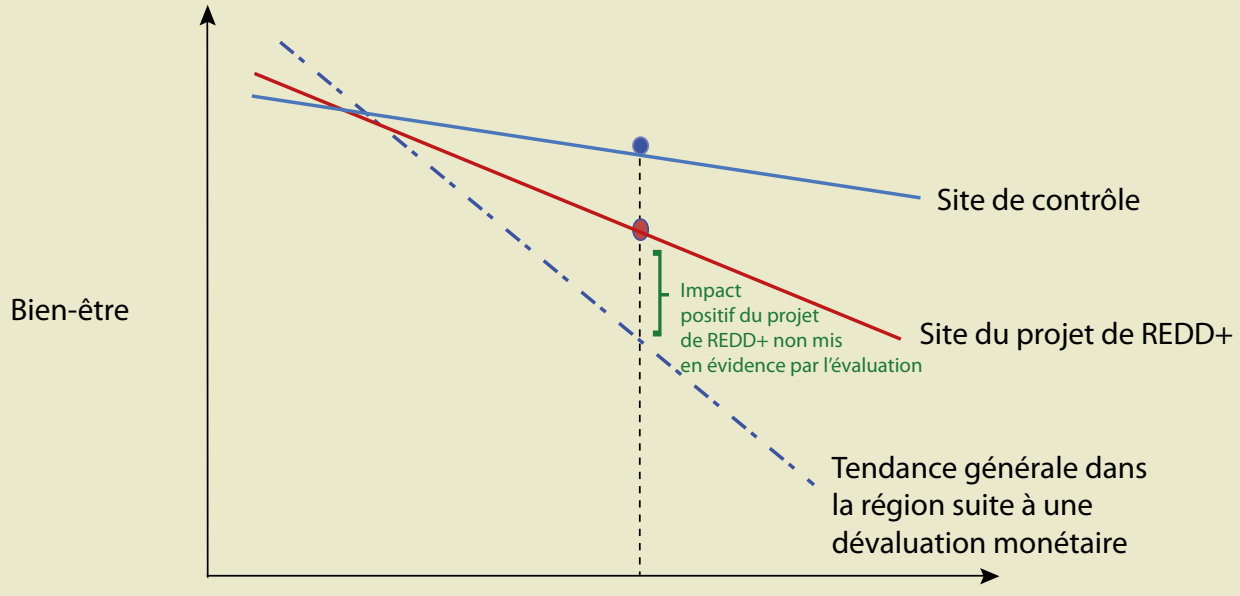

Figure 3. Attribution erronée d'une baisse du bien-être à un projet REDD+ suite à une comparaison avant-après

Imaginons maintenant une comparaison simple avec-sans au même endroit (cf. Figure 4). Le bien-être économique est mesuré une fois, cinq années après le début du projet, auprès de la population ayant bénéficié du projet de REDD+, et aussi dans des localités situées dans la même province, mais en dehors de la zone du projet de REDD+. Les données mettent en évidence un écart important de bien-être moyen entre le site du projet et le site de contrôle, celui-ci paraissant mieux loti. L'évaluation conclut également que le projet de REDD+ a été préjudiciable aux populations sur le plan économique. Cependant, lorsque les effectifs de la fonction 
publique ont été réduits, les emplois des fonctionnaires appartenant au même groupe ethnique que le président ont été épargnés. Or, ce groupe ethnique était majoritaire dans la population du groupe de contrôle, mais non dans la population du projet de REDD+. Du fait que les sites de contrôle et d'intervention n'ont pas été appariés en fonction de leur composition ethnique, la comparaison simple avec-sans attribue de manière erronée la baisse de bien-être au projet de REDD+.

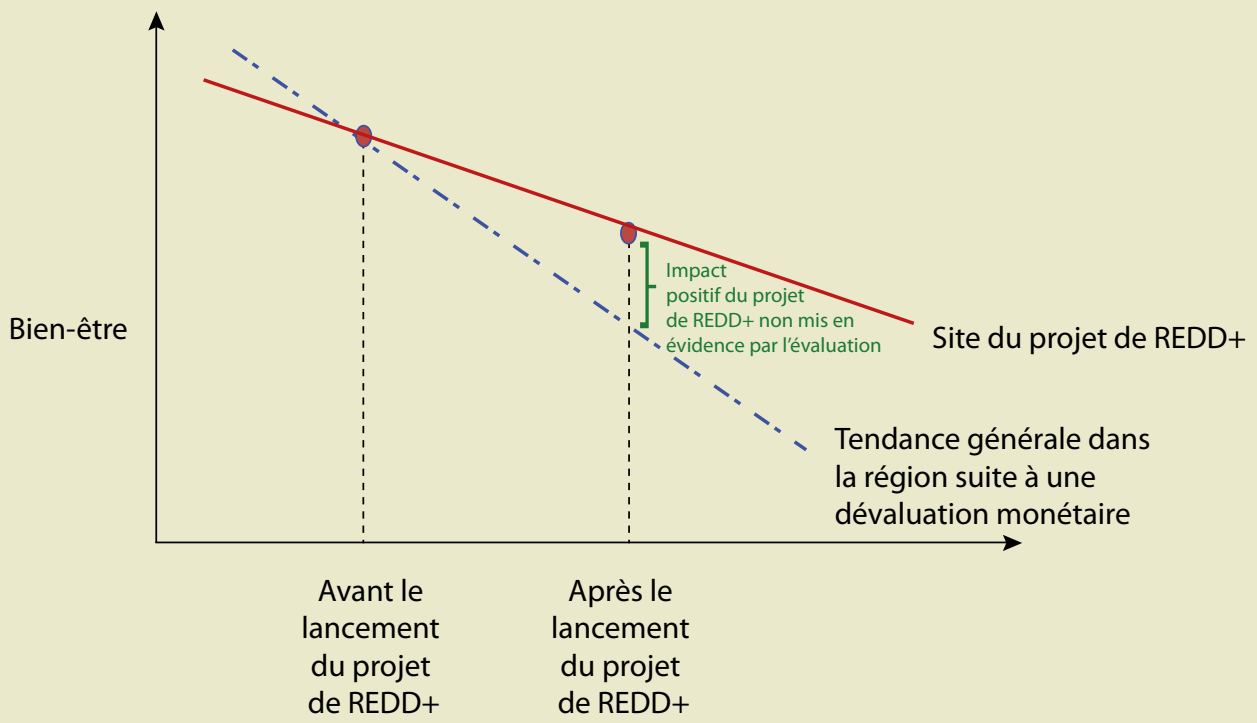

Figure 4. Attribution erronée d'une baisse du bien-être économique à un projet de REDD+ suite à une comparaison témoin-intervention sans appariement

L'appariement statistique est également utilisé après la collecte de données pour mettre en évidence les témoins les plus adaptés parmi tous les sondés. Une méthode répandue consiste à réaliser une estimation de modèle d'inclusion dans le projet (modèle de régression probit ou logit) à partir d'un grand nombre de variables recueillies par sondage, puis de réaliser un appariement selon le «score de propension » obtenu, ou la probabilité d'inclusion. L'appariement ex post est souvent effectué « avec remise ", ce qui signifie que le même témoin peut être sélectionné plusieurs fois. De cette façon, les usagers de la forêt inclus dans le projet peuvent être appariés à un sous-échantillon de témoins de taille assez réduite, qui se compose des usagers les plus semblables. Ces sous-échantillons appariés servent ensuite soit à comparer directement les résultats (test $t$ des différences de revenu moyen par ex.), soit à les calculer par des modèles de régression à variables multiples (revenu en fonction de caractéristiques des ménages et des exploitations agricoles et comme indicateur de participation au projet). Puisque c'est le sous-échantillon obtenu par appariement qui est utilisé dans le calcul de la régression, les résultats sont obtenus sans extrapolation à partir de groupes d'usagers de la forêt traités et témoins très différents.

Dans la méthodologie CI, si des données secondaires relatives à la situation antérieure au projet (issues par ex. d'un recensement ou de la télédétection) peuvent être utilisées pour sélectionner un échantillon de villages à sonder, les enquêtes pour la collecte de données relatives aux ménages sont réalisées après l'intervention. De ce fait, il est ardu de sélectionner, avant le lancement du projet, des témoins qui soient semblables aux usagers de la forêt traités, du point de vue de facteurs confusionnels ayant une influence tant sur les résultats que sur l'inclusion dans le projet, sans être eux-mêmes affectés par le projet. L'appariement dans cette méthodologie repose souvent sur des données de rétrospection (détention d'actifs avant le projet par ex.) ou des 
caractéristiques « invariables » (origine du chef de ménage par ex.), comme l'illustre la Fiche technique 7. C'est l'inconvénient potentiel majeur de la méthodologie CI : l'absence de données relatives aux facteurs de confusion principaux, qui peut provenir des difficultés liées à la reconstitution des conditions d'avant le projet ou, ce qui porte plus à conséquence, liées à l'observation de facteurs comme les préférences des ménages ou la configuration spatiale précise d'une exploitation agricole. L'Encadré 5 présente des exemples de situation contrefactuelle pouvant résulter d'un appariement inadapté entre les témoins et les traités.

Si les caractéristiques non observées des usagers de la forêt sont inchangées au cours du temps, leur influence peut être neutralisée en utilisant le plan expérimental BACI (Fiche technique 4), laquelle prévoit la sélection d'un échantillon de témoins avant l'intervention qui seront interrogés lors de la collecte des données de référence. Lors de cette phase initiale, on mesure les valeurs des critères de jugement et des facteurs confusionnels, de manière à réduire encore, le cas échéant, les effectifs de l'échantillon de contrôle. On mesure à nouveau les critères de jugement « après » le projet. Par ce procédé, on mesure deux « différences » $:$ la variation des critères de jugement entre l'avant et l'après projet, et la comparaison de cette variation entre le groupe de contrôle et le groupe traité. Cette " différence des différences » représente l'impact du projet réel, non brouillé par des différences non observées, à condition cependant que ces différences soient invariables dans le temps. Les méthodes mixtes complémentaires seront donc utiles (observation directe et entretiens sur le terrain par ex.) pour déterminer les facteurs variables dans le temps mal pris en compte par les données. Létude GCS-REDD+ du CIFOR suit une démarche BACI : les annexes de ce guide sont une mine d'informations pour ceux qui souhaitent mettre en œuvre cette méthode sur le terrain.

Pour mettre en œuvre l'approche BACI, il faut en général concevoir l'évaluation de manière anticipée, c'est-à-dire avant l'intervention ; mais dans certains cas, des données d'autres provenances (administrations, statistiques, organismes ou études) permettent d'adopter une méthodologie approchante. Comme dans le cas des expériences dans les conditions naturelles, ces données sont rarement disponibles, mais il ne faut pas négliger de les rechercher et de les exploiter. Certains projets sont mis en œuvre sur un territoire relativement vaste (ou dans une population d'usagers de la forêt assez importante) de telle sorte que des données secondaires d'enquête sont représentatives, telles que celles produites par les recensements démographiques et sanitaires (DHS, pour Demographic and Health Surveys), les Études de mesure des niveaux de vie (LSMS), ou des enquêtes propres à certains pays telles que la Pesquisa Nacional por Amostra de Domicílios (PNAD) au Brésil ou l'enquête indonésienne sur la vie de famille (IFLS ou Indonesia Family Life Survey). Ces enquêtes peuvent être utilisées :

1. si elles ont lieu avant la mise en œuvre du projet et à une date ultérieure ;

2. si elles comprennent des questions sur les facteurs de confusion et les critères de jugement pertinents.

Pour des raisons liées à l'intensité d'échantillonnage et à la confidentialité, les résultats de ce genre font normalement l'objet de comptes rendus sous forme de données plus ou moins agrégées, qui peuvent être utiles pour les très gros projets. Pour les projets de taille plus réduite, les évaluateurs peuvent parfois avoir accès à des données désagrégées de manière confidentielle. Il est également possible que la région d'implantation d'un projet soit comprise dans une aire géographique où d'autres chercheurs ont déjà collecté des données sur les usagers de la forêt. Si ces chercheurs disposent de données sur les facteurs de confusion et les critères de jugement, et sont disposés à les communiquer, la méthodologie BACI pourrait être utilisée en revisitant les mêmes ménages pour les interroger dans la phase postérieure au projet.

L'approche d'évaluation de projet ex post la plus répandue est l'analyse rétrospective ou autoréférentielle qui est réalisée uniquement à partir de données relatives aux usagers de la forêt inclus dans le projet et uniquement après la mise en œuvre de celui-ci. Cette approche est celle qui est la plus diplomatique parce qu'elle ne cherche à obtenir des informations que de personnes ayant eu la possibilité de participer et donc de tirer 
profit de la mise en œuvre du projet. L’analyse de l'impact de projet s'appuie dans ce cas sur les comptes rendus rétrospectifs d'usagers de la forêt concernant les critères de jugement avant le projet, comparativement à l'état actuel de ces critères après le projet, ou encore, sur leur propre évaluation des impacts du projet, telle qu'ils en font état. Les comptes rendus rétrospectifs peuvent être triangulés par l'utilisation de méthodes combinées, associant des entretiens auprès des ménages à des méthodes participatives et de groupe; mais ils sont plus particulièrement adaptés aux critères de jugement mesurant le sens d'une variation, des événements ponctuels de grande ampleur ou des biens (indicateurs d'actifs plutôt que mesures de la consommation ou du revenu par ex.) ; cf. Fiche technique 6. Une autre approche consisterait à utiliser les techniques de préférences déclarées à partir d'évaluations non marchandes permettant de reconstituer la valeur ou le coût de l'autre hypothèse (si le projet n'avait pas eu lieu).

Même si l'approche rétrospective ou autoréférentielle est courante et pratique, les preuves qu'elle fournit relatives aux impacts ne sont pas aussi convaincantes que les méthodes utilisant des données obtenues avant l'intervention et/ou issues de témoins ne recevant pas l'intervention. Elle ne répond guère aux besoins d'une formulation des politiques fondée sur des faits démontrés et ne permet pas de résoudre les controverses soulevées par l'appréciation des impacts de la REDD + au plan local. Dans les projets de pilotage ou de démonstration de la REDD+, nous devons nous attacher à collecter des preuves et à générer des informations sur les impacts à moyen terme de manière plus rigoureuse. Ces estimations d'impact seront essentielles à la projection des impacts à longue échéance et à grande échelle qui sont l'objectif de l'atténuation des changements climatiques sur le plan international.

\subsection{Mise en œuvre de la méthodologie de recherche}

\subsubsection{Définition de I'« avant » et de I'« après "}

De nombreux projets de REDD+ sont la continuation d'initiatives de préservation et de développement antérieures; il est donc difficile de leur attribuer un point de départ bien défini. Le début d'un projet peut être défini par la date de commencement de la période prise en compte pour l'attribution de crédits de REDD+ (pour le marché volontaire du carbone par ex.), mais il peut aussi être antérieur à cette date si l'on estime que les activités préparatoires ont eu un impact non négligeable sur le bien-être économique ou l'utilisation du sol. Pour résoudre ce problème, la date de début de projet peut définie de telle sorte qu'elle coïncide avec le début d'une intervention particulière telle qu'un système de PSE ou de surveillance et de contrôle d'exécution en temps réel, et en privilégiant l'évaluation de l'impact de cet élément particulier par rapport à celle d'autres impacts d'activités passées et en cours. Dans le cas des méthodologies de recherche BA ou BACI, cette date permettra de déterminer la période de collecte des données antérieures au projet qui se rapportent aux critères de jugement étudiés. Dans le cas des autres méthodologies, elle influera sur la collecte des données rétrospectives.

De préférence, les données rétrospectives pour l'appariement ou l'estimation des impacts doivent concerner la période située immédiatement avant le début du projet. L'utilisation de données postérieures au lancement du projet pour la définition du niveau de référence risque de conduire à une sous-estimation des effets du projet (méthode rétrospective) et d'être à l'origine d'un biais lors de la sélection des témoins (méthode contrôle-intervention). En revanche, l'utilisation de données trop anciennes par rapport au début du projet conduira à réduire la précision des estimations, mais ne créera pas de biais. Comme le montre la Fiche technique 6, le choix de la période d'obtention des données rétrospectives dépend également de facteurs pratiques : des événements notables (sécheresse, élections par ex.) peuvent améliorer l'acuité du souvenir.

La phase d' " après " peut être encore plus difficile à définir que celle d' " avant». De par leur nature, les projets de REDD+ sont dotés d'horizons très lointains (à savoir que pour recevoir des crédits carbone découlant d'une modification de la déforestation et de la dégradation des forêts, il faut faire la preuve de la « permanence» des projets). Or, si l'on veut tirer les enseignements des projets 
pilotes de REDD+, il est irréaliste de supposer que l'on peut attendre 20 ou 30 ans pour évaluer les impacts biophysiques ou sociaux des interventions. La représentation de la chaîne de causalité décrite dans la section 3 peut être utile pour situer dans l'échéancier du projet la date à laquelle les impacts devraient pouvoir être observés. Il est préférable de collecter les données relatives aux critères d'évaluation en campagnes successives afin de comprendre l'évolution des impacts au fil du temps et de changer de cap si les résultats escomptés ne sont pas observés. Cependant en raison de la nécessité d'orienter les décisions politiques à partir d'informations vérifiées, les données relatives aux résultats seront plutôt collectées assez tôt après la mise en œuvre et reflèteront de ce fait les impacts à court terme. Par exemple, l'étude GCS-REDD+ prévoit d'analyser les impacts sociaux deux années après la mise en œuvre, délai qui correspond à la période minimale d'apparition de ce type d'effet.

\subsubsection{Définition des groupes de contrôle et d'intervention}

Le Tableau 1 et la Figure 2 fournissent des repères pour l'évaluation des impacts des projets de REDD+ dans leur globalité, en supposant que les usagers de la forêt puissent être classés en participants (soumis directement aux impacts) et en non participants (non soumis aux impacts). Dans cette partie, nous étudions l'application de ces concepts à d'autres situations, en particulier à des projets consistant en de nombreuses interventions, se déroulant à des échelles très différentes, ou causant des impacts indirects ou des fuites. Pour illustrer notre propos, nous nous appuierons sur un exemple de contrats de PSE souscrits par des agriculteurs s'engageant à préserver la portion boisée de leurs exploitations, sachant que les concepts présentés peuvent être généralisés à d'autres interventions (création d'emplois locaux pour restaurer la forêt publique par ex.) et à d'autres catégories d'usagers de la forêt (ménages ramassant du bois pour la cuisine et le chauffage par ex.). Nous conclurons cette partie en recommandant des points de départ d'évaluation qui permettront de s'adapter à ces situations.

Dans le cas de projets comprenant des interventions multiples, des traitements " multiples " peuvent être définis. Par exemple, des agriculteurs ayant conclu des contrats de PSE avec le porteur de projet formeront un groupe de traitement, tandis que ceux recevant de l'assistance technique en formeront un second. Ces groupes pourront être comparés entre eux et par rapport à un troisième groupe de non participants, possédant des caractéristiques semblables mais ne recevant aucun des traitements. Même si nous nous sommes penchés sur une définition binaire du traitement pour des raisons de clarté de l'exposé, toutes les méthodes du Tableau 1 pourraient être utilisées pour évaluer des variantes d'interventions. Dans certains cas, la question n'est pas tant de connaître l'ampleur exacte de l'impact d'un projet, mais plutôt de savoir comment un projet peut parvenir à créer un impact maximal. Les méthodes expérimentales ou quasiexpérimentales permettent de répondre à cette question. Par exemple, les différentes catégories d'avantages conditionnels envisagées dans le cadre d'un projet (paiement en numéraire des ménages ou bien paiement en nature des populations) pourraient être allouées aléatoirement à différents villages dans la zone du projet. Les méthodes quasi expérimentales permettraient quant à elles de constituer des échantillons appariés d'usagers de la forêt ayant connu ces différentes options de mise en œuvre de projet. Il faut des techniques statistiques différentes pour analyser les traitements multiples et continus, mais de nombreux programmes économétriques intègrent de plus en plus ces techniques à des modules d'estimation des effets des traitements.

Dans de nombreux projets de REDD+, il existe différents degrés de traitement à des échelles différentes. Pour reprendre le même exemple, le traitement le plus « intensif » pourrait correspondre aux contrats conclus avec certains agriculteurs qui reçoivent les paiements incitatifs, participent à des programmes de formation et d'éducation et font l'objet d'une surveillance et d'un contrôle de l'exécution de leurs contrats et de l'application des lois sur la forêt. Les programmes de formation et d'éducation pourraient concerner des villages entiers (comprenant mais ne se limitant pas aux agriculteurs sous contrats), tandis que le contrôle d'exécution renforcé pourrait être étendu à toute la région (du fait de la conception du traitement ou par le biais des « retombées » de la mise en œuvre, découlant du traitement d'images de télédétection et de l'envoi de personnel de lutte 
contre les infractions dans la région pour procéder à la vérification des propriétés sous contrat). Dans cet exemple, les unités « traitées » pourraient être les agriculteurs sous contrats, les villages auxquels ils appartiennent, ou la région de mise en œuvre du projet. Le choix dépendra en partie de la définition du « projet » à évaluer : souhaitet-on évaluer l'impact des contrats de PSE dans le cadre d'un renforcement plus ou moins grand de l'information et de la lutte contre les infractions? Ou bien préfère-t-on évaluer le paquet intégral d'interventions, comprenant les contrats PSE, l'information et la lutte contre les infractions?

Les méthodes d'évaluation présentées dans ce guide sont fondées sur l'hypothèse que les autres usagers de la forêt ne sont pas affectés par le traitement des usagers inclus dans le projet (appelée en statistique « absence d'effets de diffusion du traitement » ou SUTVA). Selon la littérature traitant de l'utilisation du sol et des « fuites » de carbone, cette hypothèse est souvent démentie (cf. Annexe B.5.). En effet, les contrats de PSE qui entraînent une véritable modification de l'utilisation de la forêt et du sol chez les agriculteurs sont également susceptibles de changer les besoins de ces agriculteurs en moyens (main-d'œuvre et équipement par ex.) ainsi que leur production (cultures et élevage par ex.). Ces changements provoqueront à leur tour des répercussions chez d'autres lorsque ces personnes interagissent avec les agriculteurs participants par l'intermédiaire des marchés locaux. Les projets, en général de grande ampleur comparativement aux marchés locaux, influent aussi sur les prix ; ainsi, une intervention qui réduirait l'offre de produits agricoles provenant de la zone du projet conduirait à une hausse des prix, qui inciterait à son tour les consommateurs à passer à des produits et des producteurs de substitution dans d'autres endroits pour relancer l'offre.

En général, si un projet réduit l'activité économique et la déforestation dans la zone du projet, mais qu'il provoque des fuites de l'activité économique et de la déforestation vers les régions voisines, il en résultera une surestimation de l'impact du projet du fait de la comparaison entre l'activité dans la zone du projet et l'activité dans la région voisine. En revanche, si les bénéfices du projet conduisent à conférer à la préservation de la forêt une valeur d'option dans les zones situées à proximité, en incitant par exemple les propriétaires forestiers voisins à se positionner pour avoir aussi accès à ces bénéfices, la comparaison entre les deux zones conduira à une sous-estimation de l'impact du projet.

Afin de faire un tri entre les différentes manières de définir le " traitement » administré par un projet et d'envisager ses impacts directs et indirects, nous recommandons d'initier l'évaluation en tenant compte systématiquement des points suivants :

1. Unité d'analyse. Dans la plupart des cas, les projets s'attachent à changer le comportement des ménages. Par conséquent, l'unité d'analyse doit être le ménage, y compris ses terrains et ses activités, même si une seule parcelle seulement fait l'objet d'un contrat PSE, ou si une seule personne seulement est employée par le projet. La plupart des projets de REDD+ sont mis en œuvre dans des régions où les marchés de ressources et de produits ne sont pas complets. Dans cet environnement, les ménages ont toutes les chances de réagir à une intervention en adaptant leurs activités au sein du ménage ; par exemple, lorsqu'un ménage diminue la déforestation et la production dans un endroit donné, il sera tenté de compenser cela à un autre endroit, ce qui réduit d'autant les impacts nets sur le carbone et sur le bienêtre économique (cf. Alix-Garcia et al. 2010). La prise en compte de tout le ménage dans l'analyse permet d'internaliser ces " fuites » dans l'analyse. Certains projets de REDD+ ciblent des villages entiers, en améliorant le service public en contrepartie de la préservation des forêts publiques avoisinantes. Dans ces situations, l'unité d'analyse logique est le village ; et l'effectif de l'échantillon pour l'évaluation d'impact est exprimé en nombre de villages plutôt qu'en nombre de ménages.

2. Traitement. Dans la plupart des cas, la question essentielle concerne l'impact du paquet entier d'interventions constituant le projet. Ainsi, dans la plupart des cas, le «traitement » se définit de préférence par le paquet d'interventions pour lequel il a été prévu un financement global et un élargissement simultané de tous les éléments qui le composent. Si l'on s'intéresse plus 
particulièrement à l'efficacité respective de modalités différentes de mise en œuvre de l'intervention, il faudra prévoir d'augmenter les ressources allouées à l'évaluation ou les effectifs de l'échantillon, ou bien l'évaluateur devra choisir entre l'estimation de l'impact total de l'intervention et la comparaison de l'efficacité de chacune des modalités de mise en œuvre. Ces priorités influent sur le choix des usagers de la forêt à retenir pour former les échantillons de traitement d'une part et de contrôle d'autre part.

3. Témoins (groupe de contrôle). Les témoins du groupe de contrôle, qui ne sont pas traités directement par le projet, doivent de plus être choisis dans des villages et des zones n'interagissant pas avec les usagers de la forêt traités par le projet ; par exemple, ils ne doivent pas être susceptibles de se vendre ou de s'acheter des terrains entre eux, ni se partager ou se louer de la main-d'œuvre, ni se vendre des produits ou se transmettre des informations ou du savoir-faire. Cela signifie que les ménages du même village ou de villages limitrophes ne seront probablement pas de bons témoins, même s'il faut aussi tenir compte de la structure des réseaux sociaux et des transports locaux. Les témoins devant être semblables, notamment en ce qu'ils doivent être confrontés aux mêmes conditions biophysiques et commerciales, la recherche de témoins doit concerner en priorité les zones les plus proches du projet telles que ces témoins n'interagissent pas directement avec les usagers de la forêt traités.

4. Effets indirects. De nombreux projets auront au moins quelques effets indirects (fuite ou retombées). Les évaluations doivent analyser ces effets de manière anticipée, soit (1) par l'emploi d'une approche méthodologique mixte et la réalisation d'une analyse qualitative, soit (2) par un plan d'échantillonnage et/ou (3) soit par l'utilisation d'indicateurs obtenus par des enquêtes auprès des usagers de la forêt traités. Premièrement, il faut repérer tous les cas possibles de fuites dans le cadre de l'évaluation de la mise en ouvre du projet. Par exemple, un projet proposant des contrats de PSE en contrepartie de l'arrêt complet des coupes aura des conséquences sur le plan des fuites (déplacement de la demande vers d'autres zones et produits par ex.) différentes de celles d'un projet proposant des contrats de PSE en contrepartie de l'adoption de techniques d'exploitation forestière à impact réduit (adoption de ces techniques en retombée du projet par ex.). Deuxièmement, l'échantillonnage pourrait être élargi pour englober des usagers de la forêt non traités qui vivent ou travaillent dans la zone du projet, ou selon un gradient de distance par rapport au projet, ou encore qui sont situés dans le " périmètre de fuite ", telle que défini par le porteur de projet dans le cadre de la certification pour le marché volontaire du carbone, ce qui permettrait de rechercher des signes d'effets indirects à certaines échelles géographiques (village par ex.) ou à certaines distances (cinq $\mathrm{km}$ du projet par ex.). Troisièmement, les questionnaires d'enquête peuvent aider à mettre en évidence des effets indirects parce qu'ils interrogent les usagers de la forêt traités sur les mécanismes de fuite susceptibles de provoquer des achats de terrains ou des migrations saisonnières en dehors de la zone du projet. ${ }^{4}$

\footnotetext{
4 Lorsque que les effets indirects sont jugés préoccupants, soit en raison de leur ampleur ou parce qu'ils correspondent à un objectif clairement exprimé du projet (diffusion d'une nouvelle technologie par exemple), ils méritent une étude à part entière. Par exemple, une méthodologie expérimentale avec une randomisation à plusieurs niveaux pourrait être utilisée pour estimer les effets indirects, à condition que la configuration de ces effets soit plus ou moins connue au préalable. Ces méthodes qui dépassent le cadre de ce guide comprennent les modèles IEG qui étudient formellement la formation des prix, l'économétrie spatiale, les modèles de réseaux sociaux, d'agents ou d'effets de pairs, qui ont pour objet les interactions entre agents dans le temps et l'espace.
} 


\section{Comprendre les phénomènes de causalité reliant les interventions de REDD+ aux résultats}

\subsection{Comprendre « l'objet " et " les raisons " de l'évaluation d'impact}

J

usqu'ici notre exposé avait pour objet principal l'inférence causale intégrée à la recherche

(à savoir la randomisation ou l'emploi de méthodologies de recherche BACI, BA ou CI) et l'utilisation d'approches statistiques permettant d'éliminer les effets de facteurs de confusion et d'estimer ceux de covariables à l'intérieur d'un cadre d'évaluation d'impact. Ces méthodes représentent le moyen le plus sûr de déterminer si un projet a des impacts et de cerner l'ampleur de ces derniers. Elles peuvent également être utilisées pour étudier la variation des résultats de projet dans des groupes démographiques et socioéconomiques différents. Elles s'appuieront pour cela sur des analyses par sous-groupe ou des modèles de régression à l'aide d'échantillons rigoureusement sélectionnés (Fiche technique 9). Tous ces éléments constituent la substance, l'objet de l'évaluation d'impact.

Afin de tirer les enseignements de ces évaluations, nous devons également comprendre les raisons pour lesquelles ces impacts se sont produits ou non. Ainsi, la compréhension des causes amenant une intervention donnée de REDD+ à provoquer les conséquences observées sur le plan du bien-être économique et social passe par l'intégration de l'évaluation d'impact dans une approche méthodologique mixte, comprenant la représentation de la chaîne de causalité catalysée par la mise en œuvre du projet. Les porteurs de projet auront bien souvent adopté une démarche semblable en concevant leur projet, surtout s'ils font partie ou s'ils ont bénéficié de financements d'organismes d'aide internationale possédant leurs propres systèmes d'évaluation, listes de points à vérifier et exigences relatives aux modèles de causalité. Ces modèles de causalité comprennent les modèles logiques ( $\mathrm{l}$ ' « approche de la matrice du cadre logique ", Logical Framework Approach par ex.) (Coleman 1987, Gasper 2000, Ortengren 2004, DFID 2009), la représentation des résultats (Earl et al. 2001), les référentiels ouverts (CMP 2007) et la théorie du changement (Kusek et Rist 2004, Furman 2009) (cf. Encadré 6). Les projets visant la certification selon la CCBA ou la VCS peuvent également réaliser une représentation de la chaîne causale dans le but de mieux comprendre les impacts sociaux attendus des projets de REDD+.

Même si la représentation causale et l'évaluation rigoureuse d'impact sont couramment appliquées aux interventions dans le domaine du développement, elles sont rarement utilisées ensemble ou intégrées sous la forme d'une évaluation d'impact théorique (Reynolds 1998, White 2009). Reynolds (1998) cite les trois raisons suivantes lorsqu'il cherche à expliquer la lenteur de l'adoption des évaluations à fondement théorique.

1. La représentation de la chaîne de causalité exige souvent l'utilisation combinée de plusieurs méthodes. Les approches intégrant une analyse ethnographique et institutionnelle qualitative, des méthodes participatives et une analyse quantitatives sont en dehors du champ des méthodes conventionnelles des sciences sociales appliquées à l'évaluation de programme (dans lesquelles l'objectif de l'évaluateur a 
toujours été considéré comme étant l'évaluation de l'impact).

2. Si l'évaluation théorique n'a pas tellement intéressé les parties prenantes et les responsables politiques dans le passé, c'est en partie parce que les modèles théoriques reliant les interventions à leurs résultats ne sont pas bien au point dans un certain nombre de domaines concrets, tels que celui de la conservation et du développement.

3. Les théories sont parfois perçues négativement comme donnant des préceptes sur ce qui devrait se passer plutôt que sur ce qui se passe réellement.

Dans cette partie, nous tentons d'intégrer l'élaboration de modèles de causalité et l'évaluation d'impact rigoureuse des projets de REDD+. Employés ensemble, l'évaluation d'impact d'un côté, qui détermine le sens et l'amplitude des variations des critères de jugement principaux, et les modèles de causalité de l'autre, qui permettent de comprendre les phénomènes reliant une intervention de REDD+ à ses résultats, peuvent être des outils très puissants, les deux approches s'éclairant l'une l'autre. Cependant, nous constatons que la nature diverse des interventions de REDD+ pose des difficultés majeures pour l'évaluation d'impact théorique. Les bénéfices découlant des interventions de REDD+ sont réalisés en mettant en jeu des processus variés allant de l'appui aux collectivités locales pour la gestion des forêts et la lutte contre les infractions, à des paiements directs en numéraire aux ménages. Les projets de REDD+

\section{Encadré 6. Comparaison des modèles de causalité reliant les interventions et leurs résultats}

Les modèles logiques, la représentation des résultats, les référentiels ouverts et les méthodes des théories du changement possèdent plusieurs éléments communs. En particulier, ces méthodes permettent toutes de :

- reconnaître l'importance du contexte local et régional ;

- tenir compte de l'expérience et des opinions des parties prenantes dans les plans de surveillance et d'évaluation ;

- élaborer un modèle conceptuel de changement;

- établir quels sont les risques et les menaces et élaborer une stratégie d'atténuation ;

- privilégier la capitalisation des acquis de l'expérience par le dialogue répété avec les parties prenantes, faire un suivi permettant de repérer les succès et les risques, relier les modifications comportementales observées aux résultats constatés et rectifier le cap de la mise en œuvre en tenant compte des conclusions de ces observations.

Les cadres théoriques de référence utilisés varient également. Ainsi, certains s'attachent à imputer les résultats observés à la mise en œuvre de projet (théorie du changement par ex.), alors que d'autres s'intéressent plus particulièrement à rechercher comment le projet a changé le comportement des différents acteurs, sans toutefois chercher à formuler une théorie du changement ni à la vérifier empiriquement (représentation des résultats par ex.).

La liste ci-dessous répertorie les ressources sur Internet relatives aux modèles de causalité dans le domaine de la conservation.

- Modèles logiques http://mande.co.uk/blog/wp-content/uploads/2009/06/logical-framework.pdf

- Représentation des résultats http://www.idrc.ca/en/ev-9330-201-1-DO_TOPIC.html http://www.outcomemapping.ca/

- Référentiels ouverts http://www.conservationmeasures.org/ https://miradi.org/

- Théorie du changement http://www.iucn.org/about/work/programmes/forest/?6268/Lessons-theory-change-ME 
sont mis en œuvre par des porteurs de projet variés, allant de donateurs bilatéraux à des spéculateurs privés de la finance du carbone. La caractérisation des processus à l'œuvre dans des modèles de projet très différents est un défi de taille qu'il est essentiel de surmonter si l'on veut tirer les enseignements des projets de REDD+ (cf. Annexe B.4 qui présente des références relatives aux divers moteurs et facteurs de déforestation dans les forêts tropicales).

\subsection{Situer l'élaboration du modèle de causalité dans la conception de l'évaluation d'impact}

Dans la Section 2, nous fournissions des recommandations sur le choix d'une méthodologie de recherche pour évaluer les résultats des projets de REDD+. Les questions fondamentales sont les suivantes :

1. L'évaluation commence-t-elle avant ou après la mise en œuvre du projet ?

2. Dispose-t-on de ressources suffisantes pour collecter des données sur les usagers de la forêt ne participant pas au projet (à savoir les groupes de contrôle)?

3. La mise en œuvre du projet peut-elle être conçue de manière à faciliter l'évaluation (randomisation d'un projet mis en œuvre progressivement au niveau d'un paysage entier) ? Les réponses à ces questions ont également une incidence sur l'élaboration de modèles de causalité.

La collecte de données pour la représentation des chaînes de causalité avant et pendant la mise en œuvre de projet apporte des avantages considérables :

- Les données de la phase « avant» (méthodologie BACI ou BA) peuvent avoir une influence sur l'intervention ; un modèle de causalité peut donner des indications sur les éléments les plus intéressants à vérifier et sur la marche à suivre pour effectuer une sélection randomisée des sites de projet.

- La plupart des projets sont mis en œuvre dans des paysages hétérogènes et font intervenir des acteurs tout aussi hétérogènes. Dans les méthodologies BACI ou BA, un modèle de causalité peut indiquer là où concentrer les efforts et le mode de répartition de l'échantillon pour dégager le maximum d'enseignements.

- Un modèle de causalité doit intégrer l'évaluation des processus opératoires et la compréhension de la mise en ouvre telle qu'elle se passe réellement sur le terrain; ainsi, dans le cas des méthodologies BACI et BA, il y a souvent un modèle initial actualisé par la suite en fonction de la mise en œuvre réelle et observée sur le terrain.

- De nombreux projets sont mis en application de manière progressive. La planification initiale de l'évaluation, quelle soit expérimentale ou quelle obéisse à une méthodologie BA/BACI, doit répondre à la question de savoir comment prendre en compte les résultats d'évaluation pour éclairer les étapes ultérieures du projet.

- Les méthodologies BACI et BA offrent toutes deux la possibilité de modéliser les chaînes de causalité ex ante; la collecte de données et leur analyse en milieu de projet peuvent servir à repérer des problèmes éventuels et trouver des solutions, dont on tiendra compte pour corriger le cap et obtenir de meilleurs résultats pour la subsistance des populations.

Tout comme pour une évaluation d'impact rigoureuse, la représentation des chaînes de causalité doit faire l'objet de décisions prises au tout début du projet (à savoir avant même que l'intervention de REDD+ n'ait lieu), ceci afin de favoriser au maximum la compréhension des phénomènes de causalité en jeu.

La représentation des chaînes de causalité est assortie d'objectifs aussi bien à court terme quà long terme. L'acquisition des connaissances à court terme est favorisée au maximum lorsque les projets de REDD+ font l'objet d'un échelonnement aléatoire dans les populations et les paysages. La mise à l'épreuve des hypothèses relatives aux phénomènes de causalité dans les premières étapes du projet et l'intégration des résultats de cette opération dans les phases ultérieures de l'intervention de REDD+ peut permettre d'améliorer les résultats attendus, ce qui justifie largement les investissements réalisés pour la représentation des chaînes de causalité. Les concepts expérimentaux dans lesquels les efforts 
d'évaluation sont déployés après le début des interventions (à savoir le plan expérimental CI, ou méthodologie rétrospective) offrent également la possibilité de mieux concevoir les phases ultérieures et les projets futurs de REDD+, tout en nous permettant d'élargir notre pool de connaissances sur ce qui détermine des résultats favorables sur le plan du bien-être économique. Dans le plan expérimental CI et la méthode rétrospective, des processus théoriques et d'observation devront être intégrés au modèle de causalité lors de son élaboration.

Dans le cadre de l'étude GCS-REDD+, mais aussi probablement dans d'autres travaux d'évaluation, la production de résultats est soumise à des contraintes de temps. Par conséquent, une évaluation anticipée va porter sur des indicateurs de résultats de court terme. Un modèle causal est donc crucial pour choisir des indicateurs et réfléchir à la relation entre ces indicateurs et les résultats à long terme visés par les projets de REDD+ dans le cadre de l'objectif de " permanence ». En revanche, de nombreuses évaluations rétrospectives sont initiées longtemps après le lancement du projet ; dans ce cas, les modèles de causalité sont également très importants dans la mesure où ils permettent de reconstituer les étapes et les phénomènes intermédiaires qui auraient pu être à l'origine des résultats observés à longue échéance.

\subsection{Représenter et mettre à l'épreuve les modèles de causalité}

Létablissement d'un lien de cause à effet sans équivoque entre les résultats observés et les interventions d'un projet demande un travail de préparation considérable, ainsi qu'une collecte et une analyse de données rigoureuses. La question de savoir comment les interventions de REDD+ peuvent servir de levier du changement sur le plan local est fondamentale. Si certaines causes de la déforestation et de la dégradation des forêts et certaines évolutions du bien-être collectif sont susceptibles d'être manipulées au niveau local ou du projet, d'autres ne le sont pas (cf. Annexe B.4). Lévaluation d'impact théorique permet de replacer la capitalisation des acquis dans le cadre des limites de ce qu'une intervention de REDD+ peut accomplir et dans l'optique de la complémentarité des projets de REDD+ et des mesures politiques nationales ainsi que des grandes tendances du changement. L'évaluation théorique demande la modélisation a priori des effets attendus de l'intervention (Chen et Rossi 1983, Lipsey 1993, Reynolds 1998, White 2009). La compréhension des enchaînements des causes et des effets par lesquels les interventions de REDD+ aboutissent aux résultats permet de reproduire les réussites et de mettre en valeur les domaines où une révision et une actualisation de la mise en œuvre de projet est nécessaire.

Les modèles de causalité, ou théories du changement, sont des modèles conceptuels qui mettent en évidence les relations entre les activités de mise en œuvre, les résultats et les impacts (Tableau 2). Ils définissent les ressources, les activités et les produits de la mise en œuvre, et leurs effets à court et à moyen terme sur les critères de jugement étudiés, ainsi que les changements et les impacts à plus longue échéance (Green et Caracelli 1997, White 2009). Nous pouvons utiliser les divers éléments d'une théorie du changement pour élaborer un modèle décrivant comment les interventions conduisent aux résultats souhaités. Les modèles de causalité peuvent être qualitatifs (quand l'effectif de l'échantillon est trop réduit ou quand les variables de processus varient trop peu pour pouvoir faire une analyse quantitative par ex.). Ils peuvent aussi faire appel à la combinaison de méthodes qualitatives et quantitatives, consistant à élaborer un modèle statistique ex ante capable de déterminer l'impact social pouvant être imputé à l'intervention de REDD+.

Lélaboration d'une représentation de la chaîne de causalité comporte la succession des étapes suivantes :

1. Connaissance du contexte relatif au site du projet

2. Clarification des objectifs de l'intervention

3. Énonciation d'hypothèses vérifiables

4. Inventaire des données nécessaires

5. Mise à l'épreuve des hypothèses et actualisation des propositions initiales.

Ces cinq étapes essentielles de la chaîne de causalité représentent le processus par lequel les interventions de REDD+ entraînent une variation des critères de jugement de l'efficacité du projet 
Tableau 2. Éléments pour la représentation de la chaîne causale

\begin{tabular}{|c|c|c|c|}
\hline & Élement & Description & Application aux projets de REDD+ \\
\hline \multirow[t]{3}{*}{ Mise en œuvre } & Ressources & $\begin{array}{l}\text { Ressources entrant dans le } \\
\text { projet }\end{array}$ & $\begin{array}{l}\text { Fonds nécessaires au financement de } \\
\text { la surveillance et du contrôle relatifs à } \\
\text { l'utilisation des ressources forestières ; appui } \\
\text { à la formation en matière de gestion durable } \\
\text { des forêts; emploi de personnel local ; } \\
\text { investissements en infrastructures }\end{array}$ \\
\hline & Activités & Ce que nous faisons & $\begin{array}{l}\text { Surveiller les activités ; contrôler la mise en } \\
\text { application des règles ; former les populations } \\
\text { locales ; animer des ateliers ; construire des } \\
\text { infrastructures }\end{array}$ \\
\hline & Produits & Ce que nous produisons & $\begin{array}{l}\text { Préservation de la surface de forêt ; réduction } \\
\text { de la dégradation de la forêt ; information des } \\
\text { populations ; éléments tangibles pouvant être } \\
\text { comptés }\end{array}$ \\
\hline \multirow[t]{2}{*}{ Résultats } & Effets & $\begin{array}{l}\text { Ce que nous faisons; } \\
\text { les changements de } \\
\text { comportement découlant } \\
\text { des produits du projet }\end{array}$ & $\begin{array}{l}\text { Revenus plus importants ; meilleure santé ; } \\
\text { fourniture de services environnementaux } \\
\text { (qui contribuent tous au renforcement du } \\
\text { bien- être). }\end{array}$ \\
\hline & Impacts & $\begin{array}{l}\text { Changements durables } \\
\text { découlant d'une } \\
\text { accumulation d'effets }\end{array}$ & $\begin{array}{l}\text { Variation à la hausse ou à la baisse des } \\
\text { quartiles de revenus; entrées et sorties de } \\
\text { pauvreté ; accumulation ou perte de biens }\end{array}$ \\
\hline
\end{tabular}

Tableau adapté de Kusek et Rist (2004)

sur le plan du bien-être économique et social. Ces étapes sont décrites de manière plus détaillée dans la Fiche technique 8.

\subsubsection{Compréhension du contexte relatif au site du projet}

Selon White (2009), la connaissance du contexte et l'anticipation de l'hétérogénéité sont deux éléments essentiels de l'évaluation d'impact théorique. Le contexte influe directement sur les maillons de la chaîne de causalité. Il est constitué du cadre socioéconomique, démographique, institutionnel et biophysique dans lequel les projets sont mis en œuvre. La connaissance du contexte doit englober la description des facteurs en jeu à plusieurs échelles ; les phénomènes locaux pouvant subir l'influence d'actions entreprises au niveau infranational voire national. Avant de représenter la chaîne de causalité, les évaluateurs doivent s'assurer de bien comprendre les conditions sociales et écologiques caractéristiques du site du projet : on peut ainsi constater que les documents de conception de projet (DCP) comprennent les informations principales permettant de caractériser les conditions démographiques, socioéconomiques, institutionnelles et biophysiques propres au site. Ces informations qualitatives et quantitatives fournissent des indications intéressantes sur les conditions initiales dans lesquelles le projet va s'insérer.

Les facteurs les plus importants à connaitre au plan local comprennent :

- les moteurs de la déforestation et de la dégradation des forêts, y compris la définition de la nature des principaux acteurs influant sur les changements d'utilisation du sol (petits exploitants agricoles, éleveurs, titulaires de concession etc.) ;

- droits et régime de propriété relatifs au sol, aux arbres et au carbone ;

- degré de surveillance et de contrôle d'application, et de contestation des droits de propriété ;

- stratégies de subsistance principales dans la zone du projet ; 
- degré de dépendance des ménages à la forêt (exprimé en produits extraits de la forêt et en services fournis par cette dernière);

- hétérogénéité de la dépendance à la forêt (importance relative de la forêt pour les ménages dirigés par des femmes, les ménages de migrants etc.);

- présence et fonction des groupes ayant pour objet la gestion des ressources naturelles et le bien-être économique et social ;

- rapports établis entre le porteur de projet de REDD+ et la population.

De plus, plusieurs variables liées au contexte permettent de situer le site de projet dans un cadre plus global. L'importance du contexte est telle dans le champ de l'évaluation d'impact théorique que la même intervention mise en œuvre dans des cadres différents peut entraîner des effets différents. Nous devons être capables d'expliquer pourquoi les résultats observés diffèrent en fonction du cadre du projet, en mettant en évidence les facteurs du contexte auxquels on peut imputer la réussite ou l'échec du projet. Les données liées à des variables structurelles à une échelle plus grande permettent de situer l'analyse dans le contexte plus large du paysage infranational ou national. Elles donnent donc des informations sur le caractère généralisable d'un projet. Les variables souvent utilisées pour exprimer le caractère généralisable des conclusions d'un projet sont la nature de la forêt, le degré de transition forestière, la région agro-écologique, l'accès aux marchés, le niveau de revenu, les activités économiques principales, la densité de population et le groupe ethnique ou linguistique majoritaire.

Les informations relatives au contexte permettent de prévoir quelles seront les causes d'hétérogénéité potentielles (les impacts peuvent varier selon la conception de l'intervention, les caractéristiques des bénéficiaires ou le cadre socioéconomique). L'impact des interventions de REDD+ sur le plan du bien-être économique et social peut être différent en fonction de l'appartenance ethnique, de l'identité sexuelle, de l'âge, de l'appartenance à un groupe migrant, et de la richesse relative, entre autres. Les connaissances sur l'existence de groupes marginalisés ou vulnérables, d'élites fortunées, et d'autres groupes avant la collecte des données relatives aux critères de jugement de l'efficacité du projet permettent de définir des échantillons dont les effectifs sont représentatifs de chaque groupe lors de la conception de l'évaluation ; il est aussi souvent utile de réduire l'objet de l'analyse de la répartition à des groupes dont on sait ou dont on s'attend à ce qu'ils soient affectés par l'intervention de manière différentielle (Fiche technique 9). L'analyse ex post de l'hétérogénéité d'impact repose sur la connaissance a priori des groupes qui sont susceptibles de réagir différemment à l'intervention de REDD+. Les méthodes participatives sont particulièrement utiles pour mieux comprendre où se situent les facteurs d'hétérogénéité.

Les plans d'évaluation comprenant des groupes de contrôle doivent collecter les mêmes données de contexte pour les sites des groupes de contrôle. Ces données fournissent des variables témoins qui apporteront un éclairage sur les effets observés dans les sites d'intervention et constituent également un fondement pour l'élimination des explications contradictoires à propos des impacts observés dans les sites d'intervention, ce qui renforce la validité externe des impacts estimés sur le plan du bienêtre économique.

\subsubsection{Clarification des objectifs de I'intervention de REDD+}

Les DCP exposent en général une stratégie de mise en œuvre à court et moyen terme. La caractérisation adéquate de l'intervention repose sur notre capacité à faire la distinction entre la conception du projet et sa mise en œuvre réelle sur le terrain (cf. Encadré 7 sur les méthodes de l'étude GCS-REDD+ pour caractériser et comprendre les processus de mise en œuvre). L'évaluation d'impact théorique suppose que les objectifs du programme ont été exprimés correctement, que la mise en œuvre du programme a été vérifiée (c'est-à-dire que le projet a bien été mis en œuvre conformément au DCP) et que la théorie du programme et les phénomènes de causalité correspondants ont été définis et mesurés (Khandker et al. 2010).

Il arrive souvent que les projets de développement et de conservation ne soient pas entièrement mis en œuvre en conformité avec ce qui avait été projeté en raison de contraintes logistiques, financières et institutionnelles. Par exemple, un 
DCP pourrait décrire un système de partage des bénéfices dans le cadre de la REDD+ prévoyant le transfert de paiements carbone du porteur de projet directement vers les ménages. Cependant, les chargés de projet sur le terrain peuvent décider pour des raisons d'efficacité de créer un comité de gestion des revenus du carbone implanté dans le village qui agit comme intermédiaire entre la structure de mise en œuvre et la population locale. Si l'on se contente de ne faire qu'un suivi du budget des paiements aux ménages, on risque de ne pas faire apparaître les phénomènes de cause à effet à l'origine des changements du bien-être dans tous ses aspects. L'évaluation des processus opératoires doit être conçue de telle façon qu'elle permette d'anticiper ces modifications de la mise en œuvre du projet, en recueillant des données sur les aspects essentiels du déroulement des opérations et en utilisant des indicateurs de résultats, à même de détecter les changements prévus et imprévus relatifs au bien-être et à l'utilisation du sol. Dans les cas où la mise en œuvre s'écarterait des ressources, activités et produits décrits dans les DCP, les méthodes qualitatives fournissent un outil précieux pour générer de nouveaux éclairages et renouveler notre compréhension de la mise en œuvre. Par l'observation et des entretiens très poussés, il sera alors possible de savoir si les plans inscrits dans les DCP reflètent adéquatement la situation sur le terrain.

L'étude GCS-REDD+ du CIFOR réalise une analyse détaillée des modalités de mise en œuvre de la REDD+ et des relations entre celle-ci et l'évolution du bien-être économique et social (Encadré 7).

\section{Encadré 7. Étude de la mise en œuvre de projet dans le cadre de la GCS-REDD+}

L'étude GCS-REDD+ utilise une approche itérative de collecte d'informations sur les modalités de mise en œuvre des projets de REDD+ et les coûts qui lui sont associés. Dans la phase de l'« avant » projet, I'« évaluation du porteur de projet » ou EP (cf. I'Annexe C présentant cet outil de recherche) permet de collecter des informations essentielles, concernant l'organisation à laquelle appartient le porteur de projet, les composantes essentielles du projet de REDD+, la méthodologie relative aux activités de MNV et le FPIC, les partenaires principaux, les projets de certification et de vente de crédits et la localisation du projet. Lors de l'EP, le porteur de projet doit faire la liste des parties prenantes (groupes d'individus ou d'entreprises) qui utilisent la forêt au niveau du site du projet, et dont on prévoit qu'ils modifieront leur utilisation de la forêt dans le cadre de l'objectif de réduction des émissions de carbone du projet. Le porteur de projet doit également présenter les stratégies précises par lesquelles il espère provoquer ces modifications de comportement chez les usagers de la forêt. II s'agit en somme du modèle de causalité du projet. Par ailleurs, l'EP est conçue de telle manière à recueillir des précisions sur la sélection des villages particuliers appelés à recevoir l'intervention, ce qui permet de définir les facteurs fondamentaux à prendre en compte pour apparier les villages et les ménages traités avec les villages et sites témoins. Outre l'évaluation du porteur de projet, les chercheurs doivent rédiger un compte rendu de site, présentant les caractéristiques de la région du projet, en décrivant notamment les grands moteurs de la déforestation et la situation antérieure au projet de REDD+.

Dans la phase « intermédiaire » de la recherche, les modalités, coûts et aspects politiques de la mise en œuvre de projet font l'objet d'un suivi à l'aide de l'« étude de la mise en œuvre de projet » ou SPI (Survey of project implementation) (cet outil de recherche sera disponible sur le site Internet du CIFOR en 2011). Le SPI permet aux chercheurs de se rendre compte des activités de projet qui ont été réalisées. Pour cela, ils effectuent une double tâche qui consiste d'une part à définir les activités pouvant être imputées au projet (en déterminant par ex. si la délivrance de titres de propriété dans la zone du projet fait partie de celui-ci ou si elle est une activité complémentaire nécessaire au projet, mais qui aurait pu avoir lieu sans lui), et d'autre part celles qui ont véritablement eu lieu (et pas seulement sur le papier). Cette double tâche gagne à être effectuée pendant que le projet est mis en œuvre et sur place. Dans l'étude GCS-REDD+, le retour des chercheurs sur les sites des projet pour rendre compte de la première phase de la recherche offrira l'occasion de collecter ces informations. La SPI quantifie également les coûts de lancement du projet qui comprennent les frais de préparation, les frais administratifs et les coûts de transaction, ainsi que, le cas échéant, les dépenses courantes dans les premières étapes de la mise en œuvre. 


\subsection{3 Énonciation d'hypothèses, inventaire des données nécessaires et vérification des hypothèses}

Quelle que soit la manière dont on la formule, la question de savoir « quels sont les impacts sociaux des projets de REDD+ » conduit à émettre des hypothèses. Une hypothèse est une proposition scientifique fondée ou une approximation raisonnée cherchant à caractériser la relation qui relie deux variables. Les hypothèses répondent à deux exigences : elles doivent coller aux faits connus et elles doivent être vérifiables. L'énonciation des hypothèses soulève des questions importantes, à savoir :

- La variable peut-elle être mesurée directement, ou faut-il relever une variable de substitution à sa place?

- Les données peuvent-elles être recueillies avec le temps et les ressources dont on dispose?

- Les données présentent-elles suffisamment de variation pour pouvoir vérifier les hypothèses?

Les évaluateurs de projet qui s'engagent à réaliser l'évaluation d'impact théorique des interventions de REDD+ sont confrontés à plusieurs difficultés. La première, et la plus importante, découle de l'extrême complexité de la plupart des systèmes socioécologiques, qui rend leur compréhension particulièrement ardue (Chhatre et Agrawal 2009, Ostrom 2009). Ostrom (2009) définit ainsi, à l'aide d'un cadre théorique comportant plusieurs niveaux d'analyse des systèmes socioécologiques, plus de 40 variables qui appartiennent à des catégories différentes : systèmes de ressources, usagers et unités, systèmes de gouvernance, interactions, résultats et écosystèmes associés. La détermination des groupes de variables et de relations qui sont essentiels à la compréhension de la chaîne de causalité entre intervention et résultats observés pose donc un défi considérable.

La deuxième difficulté provient du fait que les théories du changement n'ont, dans la plupart des cas, pas fait l'objet d'une vérification rigoureuse et empirique dans le domaine de l'environnement et du développement. Schreckenberg et al. (2010) soulignent que des recherches économétriques font défaut pour mettre en évidence les liens de corrélation entre les projets de terrain dans le domaine de la conservation et du développement et les résultats concernant le bien-être économique et social ; par conséquent, il n’existe pas de modèles de causalité généraux pour la définition des indicateurs. Les résultats et inférences utilisées pour la représentation de la chaîne de causalité seront largement tributaires de la validité de la théorie du programme et de l'analyse explicative. Lorsque des théories solides ont été testées empiriquement eu égard aux éléments nécessaires à la mise en œuvre réussie d'un projet de REDD+, l'évaluation d'impact fondée sur la théorie s'en trouve facilitée. Cependant dans bien des cas, la validité des liens théoriques qui concernent spécifiquement la conservation et le développement sont contestés. Nous savons empiriquement par exemple qu'une taille ou hétérogénéité trop importante du groupe visé est un obstacle à l'action collective en faveur de la gestion durable des forêts (Poteete et Ostrom 2004), bien que ces auteurs attirent l'attention sur le caractère incertain de leurs résultats empiriques. Faute de trancher sur la question, la plupart des études récentes attisent le débat (Baland et al. 2007 concluent ainsi que l'inégalité influe de manière non linéaire sur la coopération). Afin de vérifier complètement un modèle de causalité quel qu'il soit, nous avons besoin d'une théorie bien conçue qui nous permette de poser des hypothèses relatives aux liens existant entre les interventions de REDD et les résultats sur le plan du bien-être économique et social (Reynolds 1998).

Nous donnons ci-après des exemples de « variables de processus " permettant d'examiner et d'énoncer des hypothèses au sujet des liens entre les interventions de REDD+ et les résultats :

- accès à la forêt ;

- droits de propriété et de gestion des arbres, de la forêt, et du carbone forestier ;

- participation à la conception et la mise en œuvre de projet ;

- existence, accessibilité et efficacité des systèmes de résolution des doléances;

- nature de l'initiative à l'origine du projet (approche descendante ou consultation ou consentement libre, préalable et éclairé (FPIC) ou initiative de la collectivité);

- communication des informations et sensibilisation aux activités du projet ; 
- communication des informations relatives aux flux financiers du carbone ;

- capital social ;

- dissensions au sein de la population ;

- volatilité de la finance carbone et des paiements/ bénéfices revenant à la population ;

- changement des comportements relatifs à l'utilisation de la forêt (la REDD+ est-elle en train de créer des incitations paradoxales favorisant la déforestation?)

- existence, conception et efficacité des systèmes de partage des bénéfices ;

- efficacité des mesures d'atténuation projetées pour combattre les moteurs véritables à l'origine des pertes forestières sur le site du projet ; rémunération correspondante.
Pour bien comprendre la chaîne de causalité entre intervention et résultat, il sera probablement nécessaire d'énoncer et de vérifier plusieurs hypothèses. Les phénomènes de causalité dans les systèmes écologiques et sociaux ne sont en général pas linéaires ; qui plus est, ils ne se produisent pas indépendamment les uns des autres. L'étude GCSREDD+ comprend la vérification de plusieurs hypothèses relatives aux répercussions des projets de REDD+ sur l'état des forêts et le bien-être économique des ménages (Encadré 8).

L'élaboration et la mise à l'épreuve d'une représentation de la chaîne de causalité nécessitent plus de données que les autres méthodes d'évaluation d'impact. Il peut être long et coûteux de mesurer les phénomènes de causalité qui interviennent, de définir précisément et de

\section{Encadré 8. Hypothèses fondamentales posées par l'étude GCS-REDD+}

L'étude GCS-REDD+ du CIFOR en cours met à l'épreuve plusieurs hypothèses concernant les répercussions de la conception et de la mise en œuvre des projets de REDD+ sur les forêts et le bien-être économique des ménages. Bon nombre de ces hypothèses cherchent à comprendre comment les impacts sur le bien-être économique, physique et psychologique peuvent se répercuter sur les impacts forestiers. Les hypothèses suivantes figurent parmi celles testées par l'étude GCS-REDD+ :

L'efficacité de la REDD (définie comme l'accomplissement de la réduction des émissions de carbone forestier et de l'augmentation de la séquestration du carbone) repose sur les conditions suivantes:

1. prise en compte suffisante de l'efficacité, l'efficience, l'équité et des co-bénéfices

2. détermination exacte des moteurs de la déforestation et de la dégradation

3. interventions adaptées ayant pour cible les moteurs de la déforestation et de la dégradation

4. résolution préalable des droits de propriété contestés concernant le sol, les ressources naturelles et le carbone

5. accueil favorable et participation à la REDD+ assurée au plan local, se manifestant notamment par :

a. autorisation donnée au plan local pour le projet de REDD+

b. éducation au plan local sur les changements climatiques et la REDD+

c. implication des populations locales dans la conception et la mise en œuvre de la REDD+

d. mise en œuvre dans la transparence

6. ciblage adéquat des bénéfices, notamment par l'intermédiaire de :

a. part suffisamment grande octroyée aux populations par rapport aux autres parties prenantes

b. bénéfices à l'échelon du ménage et non de la communauté

c. procédé qui veille à faire bénéficier les plus démunis et les femmes

7. répartition des bénéfices et des coûts entre les parties prenantes principales considérée comme juste, en assurant notamment que :

a. toutes les parties prenantes principales tirent des bénéfices nets du projet de REDD

b. la légitimité est favorisée parce qu'aucune partie prenante n'a une part excessive par rapport aux autres

c. aucun groupe ne reçoit de bénéfices beaucoup plus élevés que ceux des autres groupes. 
concrétiser l'exposition au traitement, de recueillir les données relatives à un grand nombre de variables de processus et de maintenir dans le temps un suivi longitudinal important auprès des participants au programme, et ce pendant toute la durée de la mise en œuvre (Reynolds 1998). Les modèles de causalité et des hypothèses précises sont particulièrement utiles quand il s'agit de recenser les données nécessaires à l'étude. Les théories du changement ou enchaînements des causes et des effets orientent vers des variables spécifiques, permettent de définir le mode de mesure des variables (à savoir par la collecte de données au moyen de méthodes quantitatives ou qualitatives), ainsi que l'échelle de collecte de ces données. Selon notre approche, il existe quatre catégories de variables principales : les variables ou critères de jugement, les variables explicatives, les variables de confusion et les variables de processus (Fiche technique 10). Afin de comprendre la suite d'événements de cause à effet depuis l'intervention de REDD+ jusqu'aux résultats sur le bien-être économique et social, il est souvent nécessaire d'adopter une approche méthodologique mixte. Les données qualitatives sont particulièrement importantes dans la mesure où les phénomènes à l'origine des impacts peuvent être de nature relativement diverse : aspects liés à la mise en œuvre du projet (taux de participation suffisant et informé des usagers locaux de la forêt par ex.), conditions institutionnelles (régime foncier, degré de transfert de pouvoir relatif à la gestion vers les autorités locales, droits de propriété etc.), et caractéristiques propres à la population (domination des élites, hétérogénéité ethnique, groupes et associations intéressés par la gestion des forêts et l'amélioration du bien-être économique et social etc.).

Si les variables de processus varient suffisamment dans l'échantillon, on pourra énoncer des hypothèses relatives à ces facteurs qui pourront ensuite être vérifiées quantitativement au moyen de techniques d'évaluation d'impact, mais s'il s'agit d'un échantillon à effectifs réduits ou si les variables de processus considérées varient peu, cela ne sera pas réalisable. Ainsi, si l'on souhaite analyser comment les droits de propriété sur le carbone forestier influent sur les résultats relatifs à la forêt et au bien-être économique, mais si aucun des villages de l'échantillon ne jouit de tels droits, ou si au contraire tous en jouissent, la variation dans l'échantillon sera insuffisante pour permettre la vérification empirique d'aucune hypothèse à ce sujet. Même dans le cas où la variation est suffisante, il peut ne pas être envisageable d'étudier d'un point de vue quantitatif les hypothèses relatives aux phénomènes de causalité dans les cas où cette analyse nécessiterait une simplification excessive ou une classification arbitraire de phénomènes complexes et de conditions institutionnelles.

Lélaboration des modèles de causalité doit tenir compte des résultats de l'intervention du point de vue de l'échelle des variations attendues, en prévoyant de faire correspondre étroitement l'échelle de l'analyse des effets et des impacts à l'échelle de mise en œuvre des activités de l'intervention. Dans le cadre des projets de REDD+ de première génération, les interventions ont été canalisées à des échelles différentes, allant de l'infranational au village et, dans le cas des projets de type PSE, à l'échelle du ménage. La collecte de données au niveau du village seulement ne saurait être suffisante pour comprendre comment chaque ménage est affecté par un projet de REDD+qui distribue des paiements directs aux ménages. Inversement, la collecte de données au niveau de chaque ménage, dans le cadre d'une intervention prévoyant d'implanter un centre de santé communautaire dans un village, est superfétatoire dans la mesure où tous les ménages bénéficieront ou auront la possibilité de bénéficier de ce nouveau service public de la même manière.

D’autres facteurs influant sur les résultats écologiques et sociaux peuvent aussi certainement intervenir; qu'ils soient externes ou internes au site du projet, il est important d'en tenir compte. De manière idéale, pour éviter au maximum que ces facteurs faussent la détermination de l'impact, les données seront collectées à la fois sur les sites de contrôle et de l'intervention de REDD+ et appariées pour des facteurs susceptibles d'affecter les résultats ; cette précaution reviendra à retrancher l'effet des facteurs de confusion potentiels. Toutefois, l'appariement peut ne pas être parfait, qu'il concerne des facteurs connus ou apparaissant plus tard. Ainsi, un biais dans les résultats ne pourra être évité par exemple dans le cas de l'augmentation 
des taux de rémunération dans le site de contrôle mais pas dans le site de REDD+ (pour des raisons non liées à la REDD+) ou de l'implantation d'un projet de développement indépendant du projet de REDD+ sur le site de REDD+ ou le site de contrôle. Il est essentiel de mener une réflexion a priori sur les différents scénarios possibles et de conserver une certaine vigilance tout au long de l'étude, de manière à être en mesure d'éliminer les explications contradictoires proposées pour rendre compte des différences de résultats observées dans le site de REDD+.

Nous avons souligné à quel point il était délicat de définir et de valider l'enchaînement de causalité reliant une intervention à ses résultats. La nature complexe des systèmes socioécologiques signifie qu'un grand nombre de variables influencent la manière dont les interventions de REDD+ opèrent des changements dans le domaine du bien-être économique, physique et psychologique. A cette complexité s'ajoute le fait que les théories relatives aux interventions ayant pour objectif la conservation et le développement n’ont pas été vérifiées par des méthodes visant à déterminer les effets de causalité. Bon nombre d'études concernant les initiatives de gestion durable des forêts, les programmes intégrés de conservation et de développement et la gestion communautaire des forêts sont en fait des études de cas, dans lesquelles les hypothèses relatives aux rapports entre les variables sont rarement clairement vérifiées. C'est pourquoi l'intégration d'une analyse des modèles de causalité dans un cadre théorique d'évaluation d'impact rigoureux est si intéressante pour de multiples praticiens de la REDD+, de la conservation et du développement. 



\section{Aspects pratiques à prendre en compte pour mieux comprendre les impacts de la REDD+ sur le bien-être économique et social}

\subsection{Budgets et capacités d'évaluation}

$\mathrm{L}$ 'évaluation des projets destinés à servir de pilote ou à but démonstratif mérite que lui soit consacrée une aide financière conséquente. Or, le plus souvent, l'évaluation constitue une part infime des budgets des projets de conservation et développement. Par ailleurs, dans le domaine de la REDD+, c'est la MNV du carbone, fort coûteuse parfois, qui est largement prioritaire. Ces deux facteurs couplés remettent en cause l'allocation de ressources suffisantes pour une évaluation des impacts sur le bien-être économique et social qui soit exigeante sur le plan de la qualité et qui se fonde sur des preuves. La collecte de données relatives à des niveaux de référence et groupes de contrôle est fréquemment considérée comme étant très onéreuse. Les compétences nécessaires à la conception des études d'évaluation, au traitement et à l'analyse des données sont également jugées comme dépassant celles du personnel travaillant sur les projets (et comme nétant pas de leur ressort). Or, l'évaluation des impacts sociaux peut contribuer de manière importante à la gestion des risques juridiques, politiques et médiatiques de la REDD+ en définissant la nature de ces impacts de manière anticipée et en en établissant la preuve. Cette évaluation complète nos connaissances relatives aux impacts des projets sur les émissions de carbone, car ces deux catégories d'impact sont liées aux décisions prises par les usagers de la forêt et par leurs comportements. Consulter Bamberger (2006), Bamberger et al. (2004) et Baker 2000 pour obtenir des recommandations sur les éléments constitutifs d'un budget d'évaluation et des techniques de réduction des coûts, ainsi que des exemples de budgets d'évaluation.

Lorsqu'ils constituent un budget d'évaluation des impacts sociaux, les porteurs de projet et les chercheurs et évaluateurs qui collaborent avec eux doivent prendre en compte plusieurs facteurs, dont l'état d'avancement de la mise en œuvre du projet (à savoir phase antérieure au projet, en cours de projet ou postérieure au projet), les capacités d'évaluation et les ressources disponibles pour cette dernière. Lorsque l'évaluation commence avant le projet lui-même, les méthodologies de recherche les plus rigoureuses peuvent être employées, mais elles peuvent aussi être les plus coûteuses. Elles consistent à recueillir des données sur les zones de traitement (et aussi de préférence sur les zones de contrôle) ou auprès des usagers de la forêt avant que ne commence l'intervention. Lorsque les données sont ainsi collectées dans les tout premiers stades du projet, il arrive qu'elles permettent de repérer un sous-échantillon de témoins bien appariés aux usagers de la forêt traités, de sorte que le recueil de données puisse être circonscrit par la suite à ces témoins appariés. Toutefois, ces méthodologies de recherche sont foncièrement coûteuses parce qu'elles s'appuient sur des séries d'observations sur le terrain échelonnées sur une longue période.

Les points de repères que nous donnons ciaprès guident dans le choix d'une méthodologie d'évaluation d'impact adaptée au budget disponible.

- Budget de montant élevé pour l'évaluation des impacts sur le bien-être économique et 
social : Réaliser des enquêtes détaillées auprès d'un grand nombre de ménages; collecter des données relatives aux sites de contrôle et d'impact ayant fait l'objet d'une sélection rigoureuse ; trianguler les résultats au moyen d'entretiens avec des informateurs principaux et des réunions dans les villages; effectuer la représentation de la chaîne de causalité avant, pendant et après l'intervention pour un certain nombre d'indicateurs qualitatifs et quantitatifs définis.

\section{- Budget de montant intermédiaire pour} l'évaluation des impacts sur le bien-être économique et social : Procéder comme pour le budget de montant élevé, mais limiter le nombre des enquêtes auprès des ménages et réaliser une stratification des échantillons par groupe d'appartenance (revenu, origine ethnique, appartenance hommes-femmes, profession par ex.) ; les résultats auront un intervalle de confiance plus réduit ; la représentation de la chaîne de causalité impliquera des vérifications d'hypothèses moins fréquentes et à partir d'un nombre plus réduit de données.

- Budget de montant faible pour l'évaluation des impacts sur le bien-être économique et social : Employer des méthodes participatives à l'échelon du village en collectant des données avant et après le projet, ou sur des sites de contrôle et d'impact, ou les deux à la fois ; utiliser des méthodes rétrospectives si des données antérieures ne sont pas disponibles; appliquer les méthodes participatives à intervalles réguliers afin de s'informer sur la mise en œuvre du projet de REDD+ et représenter la chaîne de causalité.

Les capacités d'évaluation constituent une contrainte qui rebute beaucoup de porteurs de projet. Il y a deux aspects à prendre en compte. Tout d'abord, le projet dispose-t-il de personnel ou de collaborateurs possédant les compétences et la formation nécessaires à la collecte de données requise dans le cadre d'une évaluation d'impact? Pour étudier les impacts sociaux du point de vue local, il faut collecter des données au niveau du village et du ménage, mais la conception et la mise en œuvre des enquêtes auprès des villages et des ménages demande une gamme étendue de compétences et d'aptitudes. Les ressources fournies par l'étude GCS-REDD+ du CIFOR comprennent des supports de réalisation d'enquêtes socioéconomiques (cf. Annexe C) ; consulter Angelsen et al. 2011 qui donnent des ressources supplémentaires pour l'élaboration et la mise en œuvre d'enquêtes portant sur le bien-être économique et social et la dépendance par rapport aux forêts et à l'environnement. Ensuite, il est nécessaire d'avoir des collaborateurs disponibles pour traiter et analyser les données collectées. Les compétences élémentaires nécessaires comprennent l'aptitude à manipuler, nettoyer et renseigner des séries de données, faire des calculs de statistique descriptive et exécuter des programmes de modélisation de régressions et d'appariement des données. Cf. Annexe B.1 qui donne une synthèse des ressources en ce qui concerne l'évaluation d'impact.

Nous insistons sur l'importance d'inclure l'évaluation des impacts sociaux dans le programme de conception et de mise en oeuvre du projet avant le démarrage de celui-ci, ceci permettant d'adopter l'approche la plus flexible pour l'évaluation et d'accroître la probabilité que des ressources soient investies dans l'évaluation d'impact. Nous réaffirmons que les coûts de l'estimation des impacts sociaux ont toutes les chances d'être moindres par rapport à ceux de la MNV du carbone, et que l'investissement réalisé se justifie par le fait que les impacts de la REDD+ sur les moyens de subsistance déterminent largement sa viabilité politique et sociale, ainsi que la permanence du rôle qu'elle joue dans l'atténuation des changements climatiques.

\subsection{Aspects éthiques}

Toute activité de recherche faisant intervenir des personnes est susceptible d'exposer ces personnes à des risques : les porteurs de projet, les chercheurs et les autres parties prenantes ont le devoir de les protéger contre ces risques. Le rapport Belmont (1979) propose des principes dont la validité est reconnue en matière de recherche comportementale et biomédicale ; il définit notamment trois principes qui servent de référence pour une recherche éthique sur l'humain : 
1. le respect et la protection de l'autonomie des personnes

2. ne pas faire du mal et faire du bien (garantir le bien-être des personnes)

3. la justice (à savoir la distribution équitable des coûts et des bienfaits tirés de la recherche).

Il découle en particulier de ces principes que les chercheurs doivent obtenir le consentement libre, préalable et éclairé (FPIC) des sujets potentiels avant leur participation à la recherche. Il s'agit là d'un principe fondamental, mais néanmoins complexe, de la recherche sur l'humain. D'une part, les chercheurs doivent donner suffisamment d'informations aux participants éventuels afin de leur permettre de décider en connaissance de cause s'ils souhaitent participer, mais d'autre part, en donnant trop d'informations sur les phénomènes qu'ils cherchent à étudier, les chercheurs risquent de compromettre leurs objectifs de recherche. Par exemple, dans le cadre de l'étude GCS-REDD+, les chercheurs du CIFOR tentent de juger des connaissances des participants en ce qui concerne la REDD+. Cela demande de trouver le juste équilibre entre l'information des participants éventuels sur le sujet de l'étude et la rétention de certains détails du projet de REDD+ prévu localement, car trop d'explications ne permettraient plus d'évaluer les connaissances locales sur la REDD+, ni le travail d'information des initiateurs de projets auprès de la population locale.

La confidentialité est également importante pour la recherche sur l'humain. L'application du principe de non malfaisance et de bienfaisance signifie que les chercheurs doivent évaluer les risques posés par la participation à l'étude, et protéger les personnes participantes contre ces risques. Dans le cas des projets de REDD+, on conçoit aisément que la divulgation d'informations relatives au volume de produits de la forêt récoltés illégalement ou de jugements critiques vis-à-vis de la mise en œuvre du projet crée des risques. Les évaluateurs doivent donc veiller à ce que les ménages, les informateurs principaux et les villages ne soient pas nommés individuellement dans aucun rapport et que les données soient stockées de manière sûre. Un numéro d'identification unique doit être attribué à chaque ménage, village, informateur principal et porteur de projet etc. Après la saisie des données, toutes les informations permettant de remonter jusquà des personnes précises (noms ou coordonnées GPS des ménages par ex.) doivent être supprimées dans les bases de données. Cependant, dans le cas des méthodologies de recherche BA et $\mathrm{BACI}$, les informations propres à identifier les participants doivent être conservées dans la série des données permanentes, de manière à ce que les mêmes personnes puissent être revisitées pendant la phase postérieure au projet.

L'application du principe de justice assure la distribution équitable des coûts et bénéfices du projet. Le respect de ce principe est d'autant plus important que les entretiens et réunions de longue durée prévus dans le cadre de la recherche sont assez directifs et que le temps dont disposent les participants est précieux. Le principe de bienfaisance s'appuie expressément sur la notion de bénéfice d'une façon ou d'une autre pour tous les participants au projet. Les chercheurs ont ainsi l'obligation de revenir sur les lieux de l'étude pour informer la population sur les résultats et les tenants et aboutissants de la recherche. Les usagers des ressources locales auront ainsi en main des connaissances utiles sur les résultats des projets, grâce auxquelles ils pourront préconiser des changements sociaux positifs. De nombreux chercheurs en sciences sociales considèrent qu'il est de bon aloi de dédommager les personnes qui donnent de leur temps par un petit don en argent ou en nature.

La combinaison du FPIC et du principe de bienfaisance sous-entend que les chercheurs doivent expliquer les bénéfices éventuels de leurs recherches, exercice délicat dans la mesure où il est évidemment trop tôt pour définir les avantages que les personnes vivant sur le site d'un projet de REDD+ pourraient retirer de ce projet. Les répercussions des projets sont en effet tributaires des négociations internationales sur le climat, de l'évolution du marché du carbone et des bons offices des donateurs disposés à continuer de financer les initiatives de REDD+ jusquà ce que les mécanismes de marché s'établissent d'eux-mêmes. Néanmoins, il est certain que les porteurs de projet, les organisations de la société civile, les chercheurs 
et les autres parties prenantes intéressées doivent être très vigilants à ne pas susciter trop d'attentes vis-à-vis de la REDD+, en veillant notamment à présenter les avantages potentiels des projets de REDD+ d'une manière neutre.

Enfin, nous revenons à la notion de FPIC, qui s'applique aussi bien aux projets qu’à la recherche. Par exemple, la déclaration de l'ONU de 2007 relative aux droits des personnes autochtones défend le droit des populations à donner ou non leur FPIC aux activités affectant leurs terres et leurs ressources. Les experts ont constaté que l'implication des populations locales dans les activités de surveillance et d'évaluation des impacts sociaux et environnementaux était un moyen efficace d'assurer que les impacts potentiels et réels étaient bien compris (Colchester et Ferrari 2007, Forest Peoples Programme 2008). Cette compréhension est une condition préalable du FPIC, puisque cette notion implique des échanges répétés et à double sens entre les parties grâce auxquels les nouvelles informations circulent et les autorisations pertinentes sont accordées par les populations.

De nombreux porteurs de projet cherchent par tous les moyens à impliquer les populations locales dans la conception, la mise en œuvre et la surveillance de leur projet. Jenkins (2010) définit cinq niveaux de participation à la recherche (Figure 5), qui pourraient également s'appliquer à la mise en œuvre des projets.

La participation de la population locale à l'évaluation des impacts sociaux ou à la MNV du carbone est certes une condition qui comporte des risques pour les initiateurs de projets : que se passera-t-il en effet si la population s'aperçoit qu'il existe des impacts négatifs ou se rend compte qu'elle ne va toucher qu'une fraction du prix international des compensations de la fixation du carbone? Toutefois, faire participer les populations à la recherche permet aussi de mieux comprendre les acquis du projet, tout en dissipant les malentendus et les attentes irréalistes. Enfin, nous constatons que plus la participation a une assise large, en faisant par ex. intervenir les populations locales et le porteur du projet dans l'élaboration des modèles de causalité, la définition d'indicateurs adaptés et l'interprétation des résultats, plus la qualité de la recherche sera élevée du point de vue de sa pertinence, de sa fiabilité via la neutralisation des biais et de son objectivité via l'élimination des interprétations erronées susceptibles d'être produites par l'une quelconque des parties prenantes au processus de collaboration (cf. Rao 2002 et Udry 2003).

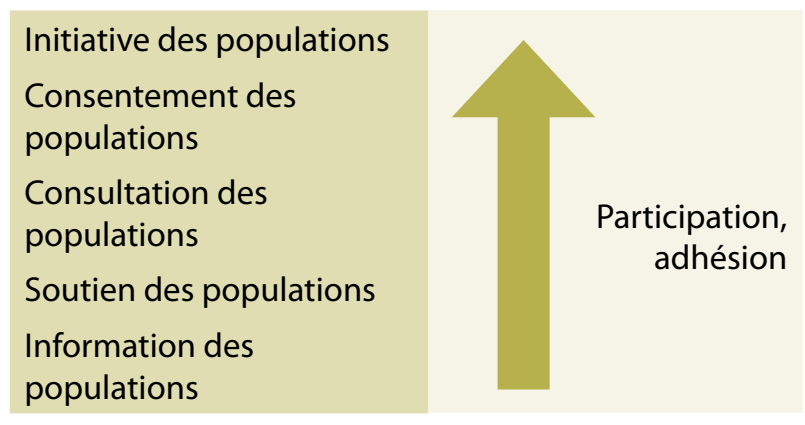

Figure 5. Niveaux de participation des populations à la recherche

Source : Jenkins (2010) 


\section{Faire avancer la réalisation de la REDD+ : principes pour mieux connaître les impacts sociaux}

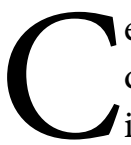

e guide est fermement attaché au concept

d'évaluation des impacts sociaux des

interventions de REDD+. Nous nous

sommes donné comme objectif de fournir les éléments théoriques et les outils pratiques dont les porteurs de projet, les donateurs, les organisations de la société civile et les usagers des ressources locales pouvaient avoir besoin pour tirer le plus d'enseignements possibles des projets de REDD+ de première génération. Nous sommes convaincus que l'évaluation des impacts sociaux ne doit pas se faire après coup ni s'ajouter secondairement à un programme de $\mathrm{M} \& \mathrm{E}$ axé uniquement sur l'évaluation des impacts biophysiques des projets de REDD+ ; elle doit au contraire être un élément central des programmes de M\&E et des budgets des porteurs de projet.

Notre étude englobe quatre éléments fondamentaux :

1. Il faut un nouvel étalon de rigueur en ce qui concerne l'évaluation des interventions dans le domaine de l'environnement et de la conservation. Les projets de REDD+ fournissent l'occasion inespérée d'appliquer une approche rigoureuse axée sur les résultats à la capitalisation des acquis, qui viendra éclairer les débats mondiaux sur la capacité des interventions de REDD+ à concrétiser les objectifs environnementaux et sociaux positifs que l'on souhaite obtenir. La REDD+ exige de par son essence même une évaluation stricte des impacts (sans laquelle on ne peut espérer vendre des crédits carbone). Les volumes des investissements consacrés aux projets de
REDD+ signifient aussi, d'une part qu'une évaluation axée sur les résultats est importante et d'autre part, que cette dernière pourra être incluse dans les budgets.

2. Lévaluation des impacts sociaux doit être fondée sur le raisonnement contrefactuel qui fait obligatoirement partie de l'évaluation des impacts biophysiques, du moins dans le sens où l'accent est mis sur ce qui se serait passé sans l'intervention de REDD+. L'avantage des méthodologies de recherche intégrant des scénarios contrefactuels est l'imputation directe des résultats observés de l'intervention de REDD+ à ses intrants. Nous avons présenté une série de méthodologies d'évaluation d'impact dans lesquelles tous les scénarios possibles de raisonnement contrefactuel sont envisagés : la randomisation est jugée comme étant l'approche optimale quand il s'agit d'imputer les résultats aux interventions; les analyses rétrospectives quant à elles, qui cherchent à reconstituer les situations contrefactuelles à l'aide de données de remémoration sont les moins fiables de toutes les méthodologies proposées. Entre ces deux extrêmes, nous étudions trois plans expérimentaux d'évaluation d'impact supplémentaires qui s'appuient sur la collecte de données " avant ", " après » et/ou de données relatives à des groupes de contrôle et d'intervention.

3. On ne peut tirer les enseignements des projets sans comprendre comment les résultats ont été obtenus. L'évaluation d'impact axée sur les résultats est une mine d'informations sur 
les répercussions réelles d'une intervention. Les enseignements des projets de première génération, et en particulier les leçons utiles pour l'élargissement de la REDD+à des niveaux d'intervention infranationaux et nationaux, ne peuvent être générés que par l'analyse des causes des changements observés du point de vue du bien-être économique et social. Les initiatives de REDD+ futures devront s'appuyer sur une étude approfondie des phénomènes de causalité à l'origine des résultats observés. Lélaboration et la représentation d'une chaîne de causalité et la mise à l'épreuve de théories du changement qui s'appuient sur des données tant qualitatives que quantitatives sont le meilleur moyen de savoir quels phénomènes précis expliquent les résultats observés.

4. Nous avons proposé des recommandations sur des aspects importants comme le calcul budgétaire, les capacités d'évaluation et les aspects éthiques liés à l'évaluation des impacts des projets de REDD+ sur le bienêtre économique et social. Nous soutenons qu'une connaissance plus approfondie des impacts de la REDD+ sur le bien-être collectif est essentielle pour mieux concevoir des initiatives futures de REDD+ qui seront durables et équitables. Lévaluation des impacts sociaux mérite d’être financée. Nous avons présenté les principes d'une approche éthique de l'évaluation des projets de REDD+. Il faut veiller attentivement à protéger les usagers locaux de la forêt vivant dans les sites d'intervention de REDD+, mais aussi ceux qui se trouvent dans les sites de contrôle, contre les risques inhérents à la participation à des activités d'évaluation.

Selon nos meilleures estimations, il y aurait environ 150 projets de REDD+ en phase de planification qui sont répartis dans les pays en développement. Ces projets posent les bases de futurs programmes et politiques d'atténuation des changements climatiques via des interventions concernant les forêts. Les travaux M\&E réalisés par les initiateurs de projets, chercheurs et évaluateurs en conservation et en développement, ainsi que dans le cadre de projets liés au carbone forestier, n'ont pas produit de principes cohérents permettant de départager les approches efficaces de celles

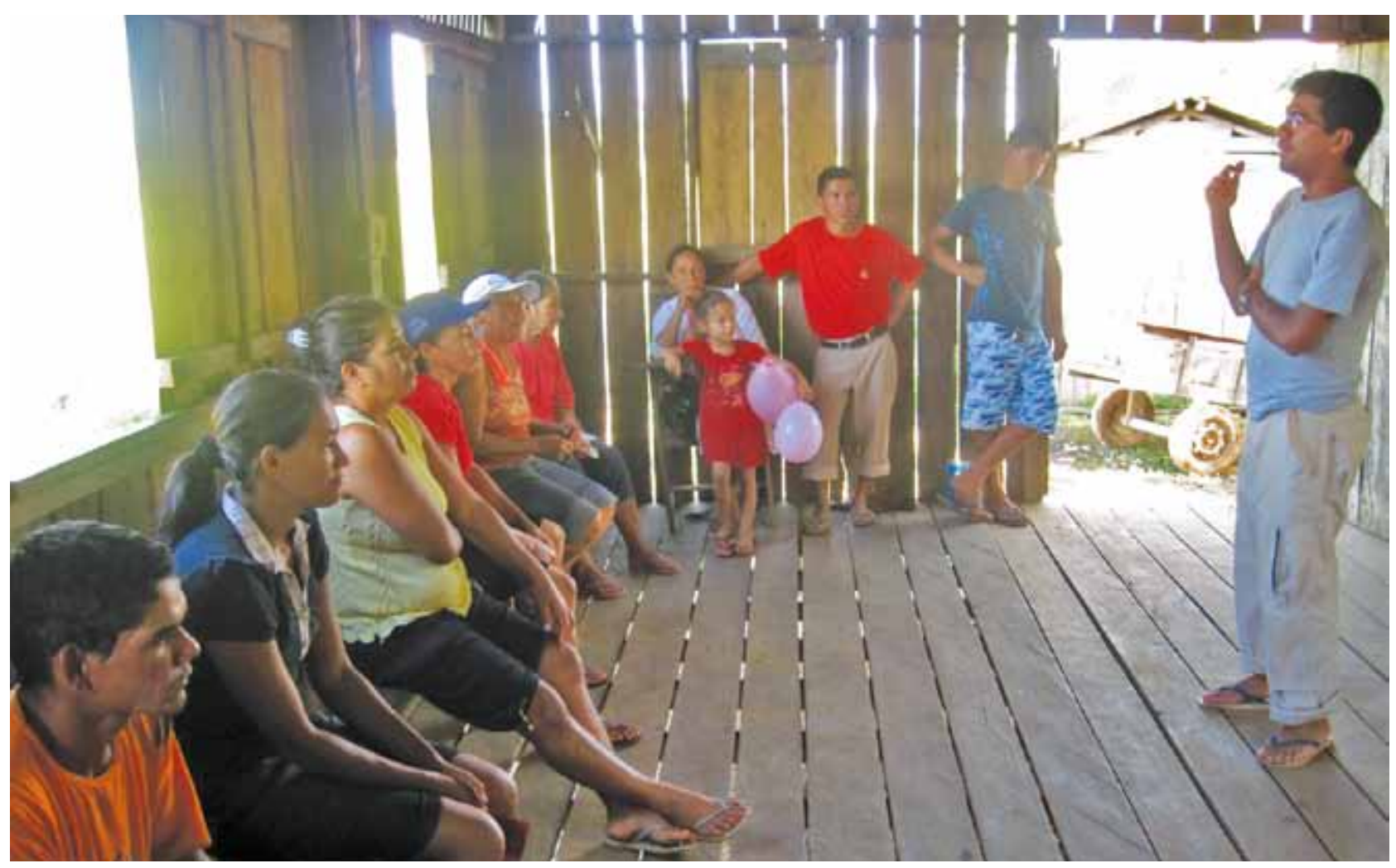

Tadeu Melo, responsable de recherche terrain, rencontre des habitants de Barro Alto dans I'Etat de l'Acre au Brésil : ces derniers sont interrogés dans le cadre d'une enquête auprès des villageois menée pour GCS-REDD+ (@ Amy Duchelle/CIFOR) 
qui ne le sont pas, s'agissant de la réduction de la déforestation et de la dégradation des forêts tout en ne portant pas atteinte ou en naméliorant pas le bien-être économique des usagers locaux de la forêt. L'impulsion donnée au plan mondial à la validation du bien-fondé du concept, via les projets de REDD+ de première génération, requiert l'engagement concerté de tous en faveur d'une appropriation rigoureuse des acquis.

Nous terminons en soulignant la nature extrêmement hétérogène du champ des projets de REDD+, lesquels sont menés par des porteurs de projet très divers et mis en œuvre par l'intermédiaire de toute une gamme de stratégies et d'accords de partage des bénéfices. Cette diversité exige l'application de méthodologies rigoureuses, aptes à pénétrer au cœur de la question de l'attribution (à savoir, quel est l'impact précis de ce projet de REDD+ sur le bien-être économique, physique et psychologique des populations locales?), ainsi que l'analyse des causes des effets observés des projets. L'agrégation des informations relatives à l'attribution, ainsi que la connaissance des causes des succès ou des échecs relatifs des interventions de REDD+ nous donneront à tous des éléments de réponse à la question fondamentale de savoir comment faire avancer la réalisation de la REDD+. Une initiative mondiale de capitalisation des acquis s'impose. 


\section{Bibliographie}

Adato, M., Carter, M.R. et May, J. 2006 Exploring poverty traps and social exclusion in South Africa using qualitative and quantitative data. Journal of Development Studies, 42(2): 226-247.

Aldrich, M. et Sayer, J. 2007 In practice: landscape outcomes assessment methodology (LOAM). Programme Des forêts pour la vie du WWF (WWW Forests for Life Programme). http://assets.panda.org/downloads/ loaminpracticemay07.pdf (consulté en novembre 2010).

Alix-Garcia, J.M., Shapiro, E.N. et Sims, K.R.E. 2010 Forest conservation and slippage: evidence from Mexico's national payments for ecosystem services program. Document de travail. https:// www.amherst.edu/media/view/209730/original/ Alix-Garcia\%2BShapiro\%2Band\%2BSims\%2 BPES\%2BMexico\%2B8-6-10.pdf (consulté en novembre 2010).

Alkire, S. and Santos, M.E. 2010 Acute multidimensional poverty: a new index for developing countries. Document de travail $\mathrm{n}^{\circ} 38$. Oxford Poverty and Human Development Initiative (OPHI), Université d'Oxford, Royaume-Uni.

Alliance Climat, Communauté et Biodiversité 2010 Standards Climat, Communauté et Biodiversité pour projets en phase d'élaboration. http://www. climate-standards.org/standards/index.html (consulté en novembre 2010).

American Carbon Registry (ACR) 2010 American Carbon Registry methodology for REDD avoiding planned deforestation, version 1.0. Winrock International, Little Rock, Arkansas, États-Unis.

Andam, K.S., Ferraro, P.J. et Holland, M.B. 2009 What are the social impacts of land use restrictions on local communities? Empirical evidence from Costa Rica. Communication présentée au Congrès de l'Association internationale des économistes agricoles, Beijing, Chine, 16 au 22 août 2009.

Andam, K.S., Ferraro, P.J., Pfaff, A., SanchezAzofeifa, G.A. et Robalino, J.A. 2008 Measuring the effectiveness of protected area networks in reducing deforestation. Actes de l'Académie Nationale des Sciences des États-Unis, 105(42): 16089-16094.

Andam, K.S., Ferraro, P.J., Sims, K.R.E., Healy, A. et Holland, M.B. 2010. Protected areas reduced poverty in Costa Rica and Thailand. Actes de l'Académie Nationale des Sciences des États-Unis, 107(22): 9996-10001.

Angelsen, A. 1995 Shifting cultivation and 'deforestation': a study from Indonesia. World Development, 23(10): 1713-1729.

Angelsen, A. 2008a REDD models and baselines. International Forestry Review, 10(3): 465-475.

Angelsen, A. 2008b How do we set the reference levels for REDD payments? Dans : Angelsen, A. (éd.). Moving ahead with REDD: issues, options and implications. CIFOR, Bogor, Indonésie, p. 53-64.

Angelsen, A. 2009 Introduction. Dans : Realising REDD+: national strategy and policy options. Angelsen, A. et al. (éd.). CIFOR, Bogor, Indonésie.

Angelsen, A. et Kaimowitz, D. 1999 Rethinking the causes of deforestation: lessons from economic models. The World Bank Research Observer, 14(1): 73-98. Banque mondiale, Washington, DC. Angelsen, A. et Wunder, S. 2003 Exploring the forest-poverty link: key concepts, issues and 
research implications. Document occasionnel $\mathrm{n}^{\circ}$ 40. CIFOR, Bogor, Indonésie.

Angelsen, A., Brown S., Loisel C., Peskett L., Streck C. et Zarin, D. 2009. Reducing emissions from deforestation and forest degradation (REDD): an options assessment report. Meridian Institute, Washington, DC.

Angelsen, A., Olsen, C.S., Larsen, H.O., Lund, J.F. et Wunder, S. (éd.) [sous presse] Measuring livelihoods and environmental dependence: methods for research and fieldwork. Earthscan, Londres.

Angelsen, A., Streck, C., Peskett, L., Brown, J. et Luttrell, C. 2008 What is the right scale for REDD? The implications of national, subnational and nested approaches. CIFOR InfoBrief $\mathrm{n}^{\circ} 15$. CIFOR, Bogor, Indonésie.

Angrist, J.D. et Pischke, J.S. 2008 Mostly harmless econometrics: an empiricist's companion. Princeton University Press, Princeton, New Jersey, États-Unis.

Appiah, M., Blay, D., Damnyag, L., Dwomoh, F.K., Pappinen, A. et Luukkanen, O. 2009 Dependence on forest resources and tropical deforestation in Ghana. Environment, Development and Sustainability, 11: 471-487.

Araujo, C., Araujo Bonjean, C., Combes, J.-L., Combes Motel, P. et Reis, E.J. 2009 Property rights and deforestation in the Brazilian Amazon. Ecological Economics, 68: 2461-2468.

Arnold, J.E.M. 2002 Clarifying the links between forests and poverty reduction. International Forestry Review, 4(3): 231-233.

Arriagada, R.A. 2008 Private provision of public goods: applying matching methods to evaluate payments for ecosystem services in Costa Rica. Thèse de $\mathrm{PhD}$. Université de l'état de Caroline du Nord, Raleigh, Caroline du Nord, États-Unis.

Asner, G.P., Knapp, D.E., Broadbent, E.N., Oliveira, P.J.C., Keller, M. et Silva, J.N. 2005 Selective logging in the Brazilian Amazon? Science, 310: 480-482.

Bajracharya, D. 1983 Deforestation in the food/fuel context: historical and political perspectives from Nepal. Mountain Research and Development, 3(3): 227-240.

Baker, J. 2000 Evaluating the impact of development projects on poverty: A handbook for practitioners, Banque mondiale, Washington, DC.

Baker, T.R., Jones, J.P.G., Rendon Thompson, O.R., Cuesta, R.M.R., del Castillo, D., Aguilar, I.C., Torres, J. and Healey, J.R. 2010 How can ecologists help realise the potential of payments for carbon in tropical forest countries? Journal of Applied Ecology, 47: 1159-1165.

Baland, J-M., Bardhan, P. and Bowles, S. 2007 Introduction. Dans : Inequality, cooperation, and environmental sustainability. Princeton University Press, Princeton, New Jersey, États-Unis.

Bamberger, M. 2006 Conducting quality impact evaluations under budget, time and data constraints. Banque mondiale, Washington, DC.

Bamberger, M. 2009 Strengthening the evaluation of programme effectiveness through reconstructing baseline data. Journal of Development Effectiveness, 1(1): 37-59.

Bamberger, M., Rugh, J., Church, M. et Fort, L. 2004 Shoestring evaluation: designing impact evaluations under budget, time, and data constraints. American Journal of Evaluation, 25(1): 5-37.

Bandyopadhyay, S. et Tembo, G. 2009 Household welfare and natural resource management around national parks in Zambia. Document de travail consacré à la recherche sur les politiques $n^{\circ} 4932$. Banque mondiale, Washington, DC.

Banque mondiale 2009 Making smart policy: using impact evaluation for policy making case studies on evaluations that influenced policy. Doing Smart Impact Evaluation No. 14. http:// siteresources.worldbank.org/INTISPMA/ Resources/383704 1146752240884/Doing_ie_ series_14.pdf (consulté en novembre 2010).

Barbier, E.B. 2001 The economics of tropical deforestation and land use: an introduction to the special issue. Land Economics, 77(2): 155-171.

Barbier, E.B. et Benhin, J.K.A. 2001 The effects of the structural adjustment program on deforestation in Ghana. Agricultural and Resource Economics Review, 30(1): 66-88.

Barbier, E.B. et Burgess, J.C. 1996 Economic analysis of deforestation in Mexico. Environment and Development Economics, 1:203-239.

Barbier, E.B., Bockstael, N., Burgess, J.C. et Strand, I. 1995 The linkages between the timber trade and tropical deforestation - Indonesia. World Economy, 18(3): 411-442.

Barrett, C.B., Marenya, P., McPeak, J., Minten, B., Murithi, F., Oluoch-Kosura, W., Place, F., Randrianarisoa, J., Rasambainarivo, J., et Wangila, J. 2006 Welfare dynamics in rural Kenya and Madagascar. Journal of Development Studies, 42(2): 248-277.

Beckett, M., Da Vanzo, J., Sastry, N., Panis, C. et Petersonet, C. 2001 The quality of 
retrospective data. Journal of Human Resources, 36(3): 593-625.

Bento, A., Towe, C. et Geoghegen, J. 2007 The effects of moratoria on residential development: evidence from a matching approach. American Journal of Agricultural Economics, 89(5): 1211-1218.

Bernard, H.R., Killworth, P., Kronenfeld, D. et Sailer, L. 1985 On the validity of retrospective data: the problem of informant accuracy. Annual Review of Anthropology, 13: 495-517.

Blom, B., Sunderland, T. et Murdiyarso, D. 2010 Getting REDD to work locally: lessons learned from integrated conservation and development projects. Environmental Science and Policy, 13(2): 164-172.

Boucher, S., Stark, O. et Taylor, J.E. 2005 A gain with a drain? Evidence from rural Mexico on the new economics of the brain drain. Département d'économie agricole et des ressources, Université de California-Davis, Californie, États-Unis.

Brown, S., Hall, M., Andrasko, K., Ruiz, F., Marzoli, W., Guerrero, G., Masera, O., Dushku, A., DeJong, B. et Cornell, J. 2007 Baselines for landuse change in the tropics: application to avoided deforestation projects. Mitigation and Adaptation Strategies for Global Change, 123(86): 1001-1026.

Bruner, A.G., Gullison, R.E., Rice, R.E. et da Fonseca, G.A.B. 2001 Effectiveness of parks in protecting tropical biodiversity. Science, 291: 125-128.

Bush, G., Nampindo, S., Aguti, C. et Plumptre, A. 2004 The value of Uganda's forests: a livelihoods and ecosystems approach. Rapport de la Wildlife Conservation Society, Programme de conservation et de gestion des ressources forestières de l'Union européenne et Uganda National Forest Authority. http://programs. wcs.org/portals/49/media/file/sForests.pdf (Novembre 2010).

Butler, R.A. et Lawrence, W.F. 2008 New strategies for conserving tropical forests. Trends in Ecology and Evolution, 23(9): 469-472.

Caplow, S., Jagger, P., Lawlor, K. et Sills, E. [sous presse] Evaluating the land use and livelihood outcomes of early forest carbon projects: lessons for REDD+. Environmental Science and Policy.

Carbon Offset Research and Education (CORE) 2010 Carbon Offset Research and Education, Stockholm Environment Institute. http:// www.co2offsetresearch.org (consulté en novembre 2010).
CARE 2002 Household livelihood security assessments: a toolkit for practitioners. Préparé pour l'unité PHLS de CARE États-Unis par TANGO International Inc., Tucson, Arizona, États-Unis. http://www.proventionconsortium. org/themes/default/pdfs/CRA/HLSA2002_meth. pdf (consulté en novembre 2010).

Carney, D. (éd.) 1998 Sustainable rural livelihoods: what contribution can we make? Department for International Development (DFID), Londres.

Carter, M.R. et Barrett, C.B. 2006 The economics of poverty traps and persistent poverty: an assetbased approach. Journal of Development Studies, 42(2): 178-199.

Carter, M.R. et May, J. 2001 One kind of freedom: poverty dynamics in post-apartheid South Africa. World Development, 29(12): 1987-2006.

Castella, J.-C., Kam, S.P., Quang, D.D., Verburg, P.H. et Hoanh, C.H. 2007 Combining top-down and bottom-up modeling approaches of land use/cover change to support public policies: application to sustainable management of natural resources in northern Vietnam. Land Use Policy, 24: 531-545.

Catley, A., Burns, J., Adebe, D. et Suji, O. 2007 Participatory impact assessment: a guide for practitioners. Feinstein International Center, Tufts University, Medford, Massachusetts, États-Unis. http://www.reliefweb.int/rw/lib.nsf/db900SID/ SHIG-7L2K8C?OpenDocument (consulté en novembre 2010).

Cavendish, W. 2000 Empirical regularities in the poverty-environment relationship of rural households: evidence from Zimbabwe. World Development, 28(11): 1979-2003.

Cavendish, W. 2002 Quantitative methods for estimating the economic value of resource use to rural households. In Campbell, B.M. et Luckert, M.K.L. (éd.) Uncovering the hidden harvest: valuation methods for woodland and forest resources, p. 17-65. Earthscan, Londres.

Cavendish, W. et Campbell, B.M. 2008 Poverty, environmental income and rural inequality: a case study from Zimbabwe. Dans : Dewees, P. et Campbell, B.M. (éd.) Managing the Miombo woodlands of southern Africa: policies, incentives and options for the rural poor. Banque mondiale, Washington, DC.

Caviglia-Harris, J.L. et Harris, D.W. 2005 Examining the reliability of survey data with remote sensing and geographic information systems to improve deforestation modeling. Review of Regional Studies, 35: 187-205. 
Caviglia-Harris, J.L. et Harris, D.W. 2008 Integrating survey and remote sensing data to analyze land use at a fine scale: insights from agricultural households in the Brazilian Amazon. International Regional Science Review, 31: 115-137.

Cerbu, G., Minang, P.A., Swallow, B. et Meadu, V. 2009 Global survey of REDD projects: what implications for global climate objectives? ASB Policy Brief $\mathrm{n}^{\circ} 12$. ASB Partnership for the Tropical Forest Margins, Nairobi, Kenya. http:// www.asb.cgiar.org/pdfwebdocs/ASBPB12.pdf.

Chambers, R. et Conway, G. 1992 Sustainable rural livelihoods: practical concepts for the 21st century. Institute of Development Studies, Brighton, Royaume-Uni.

Chen, H et Rossi, P.H. 1983 Evaluating with sense: the theory driven approach. Evaluation Review, 7: 283-302.

Chhatre, A. et Agrawal, A. 2009 Synergies and trade-offs between carbon storage and livelihood benefits from forest commons. Actes de l'Académie des Sciences des États-Unis, 106: 17667-17670.

Chomitz, K., Balmford, A., Whitten, T., Richards. M. et Berlin, A. 2007 At loggerheads? Agricultural expansion, poverty reduction, and environment in tropical forests. World Bank Policy Research Report. Banque mondiale, Washington, DC. http://go.worldbank.org/TKGHE4IA30.

Climate Funds Update http://www. climatefundsupdate.org (consulté en novembre 2010).

Cohen, M. et Barnes, C. 1996 Assets and the impact of microenterprise finance programs. AIMS Project. Management Systems International, Washington, DC.

Colchester, M. et Ferrari, M.F. 2007 Making FPIC work: challenges and prospects for indigenous peoples. Forest Peoples Programme, Moreton-inMarsh, Royaume-Uni.

Coleman, G. 1987 Logical framework approach to the monitoring and evaluation of agricultural and rural development projects. Project Appraisal, 2(4): 251-259.

Colfer, C.J.P., Brocklesby, M.A., Diaw, C., Etuge, P., Günter, M., Harwell, E., McDougall, C., Porro, N.M., Porro, R., Prabhu, R. et al. 1999 The BAG: basic assessment guide for human well-being. Manuel de critères et indicateurs $n^{\circ} 5$. CIFOR, Bogor, Indonésie.

Colfer, C.J.P., Brocklesby, M.A., Diaw, C., Etuge, P., Günter, M., Harwell, E., McDougall, C., Porro,
N.M., Porro, R., Prabhu, R. et al. 1999 The Grab Bag: supplementary methods for assessing human well-being. Manuel de critères et indicateurs $n^{\circ} 6$. CIFOR, Bogor, Indonésie.

Commission nationale pour la protection des sujets humains de la recherche biomédicale et comportementale (États-Unis) (National Commission for the Protection of Human Subjects of Biomedial and Behavioral Research) 1979 The Belmont Report: ethical principles and guidelines for the protection of human subjects of research. http://ohsr.od.nih.gov/guidelines/ belmont.html (consulté en novembre 2010).

Compliance Advisor Ombudsman (CAO) 2008 Participatory water monitoring: a guide for preventing and managing conflict. CAO, Washington, DC. http://www.cao-ombudsman. org/howwework/advisor/documents/ watermoneng.pdf (consulté en novembre 2010).

Conservation Measures Partnership (CMP). 2007 Open standards for the practice of conservation. http://www.conservationmeasures. org/wp-content/uploads/2010/04/CMP_ Open_Standards_Version_2.0.pdf (consulté en novembre 2010).

Coria, J., Hennlock, M., Löfgren, A., Persson, M., Söderholm, P., Sterner, T. et Wrake, M. 2010 The progress of GHG markets: opportunities and risks. Gothenburg Environmental Economics Report. http://www.efdinitiative.org/research/ publications/publications-repository/theprogress-of-ghg-markets-opportunities-andrisks/files/TheProgessOfGHGMarkets20100928. pdf (consulté en novembre 2010).

Costello, C., Gaines, S.D. et Lynham, J. 2008 Can catch shares prevent fisheries collapse? Science, 321(5896): 1678-1681.

Curran, L.M., Trigg, S.N., McDonald, A.K., Astiani, D., Hardiono, Y.M., Siregar, P., Caniago, I. et Kasischke, E. 2004 Lowland forest loss in protected areas of Indonesian Borneo. Science, 303(5660): 1000-1003.

Davies, R. 1997 Beyond wealth ranking: the democratic definition and measurement of poverty. http://www.mande.co.uk/docs/democrat. htm (consulté en novembre 2010).

Davies, R. et Dart, J. 2005 Most significant change (MSC) technique: a guide to its use. http:// mande.co.uk/docs/MSCGuide.pdf (consulté en novembre 2010).

De Wasseige, C., Devers, D., de Marcken, P., Eba’a Atyi, R., Nasi, R. et Mayaux, P. (éd.) 2009 The forests of the Congo Basin: state of the 
forest 2008. Office des publications de l'Union européenne, Luxembourg. http://carpe.umd.edu/ resources/sof/ (consulté en novembre 2010),

DeFries, R.S., Rudel, T., Uriarte, M. et Hansen, M. 2010 Deforestation driven by urban population growth and agricultural trade in the twenty-first century. Nature Geoscience, 3: 178-181.

Department for International Development (DFID) 2009 Guidance on using the revised logical framework. DFID Practice Paper. DFID, Londres.

Earl, S., Carden, F. et Smutylo, T. 2001 Outcome mapping: building learning and reflecting into development programs. International Development Research Centre, Ottawa, Canada.

Ecosecurities 2010 The forest carbon offsetting report 2010. http://www.ecosecurities.com/ Registered/ForestCarbonOffsettingReport2010. pdf (consulté en novembre 2010).

Estrada, M. 2011 Standards and methods available for estimating project-level REDD+ carbon benefits: reference guide for project developers. Document de travail ${ }^{\circ} 52$. CIFOR, Bogor, Indonésie.

Evans, T.P. et Kelley, H. 2004 Multi-scale analysis of a household level agent-based model of land cover change. Journal of Environmental Management, 72: 57-72.

Fairhead, J. et Leach, M. 1998 Reframing deforestation: global analyses and local realities with studies in West Africa. Routledge, Londres.

Ferraro, P.J. 2009 Counterfactual thinking and impact evaluation in environmental policy. New Directions for Evaluation, 122: 75-84.

Ferraro, P.J. et Pattanayak, S.K. 2006 Money for nothing? A call for empirical evaluation of biodiversity conservation investments. Public Library of Science Biology, 4(4): 0482-0488.

Filmer, D. et Pritchett, L.H. 2001 Estimating wealth effects without expenditure data-or tears: an application to educational enrollments in states of India. Demography, 38(1): 115-132.

Fisher, M. 2004 Household welfare and forest dependence in southern Malawi. Environment and Development Economics, 9: 135-154.

Fitzherbert, E.B., Struebig, M.J., Morel, A., Danielsen, F., Bruhl, C.A., Donald, P.F. et Phalan, B. 2008 How will oil palm expansion affect biodiversity? Trends in Ecology and Evolution, 23(10): 538-545.

Fonds International de Développement Agricole (FIDA) 2009 Evaluation manual: methodology and processes. Bureau de l'évaluation du FIDA, Rome, Italie. http://www.ifad.org/evaluation/ process_methodology/index.htm (consulté en novembre 2010).

Fonds pour l'Environnement Mondial (FEM) 2010 Developing an experimental methodology for testing the effectiveness of payments for ecosystem services to enhance conservation in productive landscapes in Uganda. http:// www.thegef.org/gef/node/2772 (consulté en novembre 2010).

Forest Peoples Programme 2008 Free, prior and informed consent and the roundtable on sustainable palm oil: a guide for companies. Forest Peoples Programme, Moreton-in-Marsh, Royaume-Uni.

Furman, R. 2009 Lessons from implementing a theory of change: M\&E system in the livelihoods and landscapes strategy initiative. UICN, Gland, Suisse.

Gasper, D. 2000 Evaluating the logical framework approach towards learning-oriented development evaluation. Public Administration and Development, 20(1): 17-28.

Gaveau, D.L.A., Epting, J., Lyne, O., Linkie, M., Kumara, I., Kanninen, M. et Leader-Williams, N. 2009a Evaluating whether protected areas reduce tropical deforestation in Sumatra. Journal of Biogeography, 36(11): 2165-2175.

Gaveau, D.L.A., Wandono, H. et Setiabudi, F. 2007 Three decades of deforestation in southwest Sumatra: have protected areas halted forest loss and logging, and promoted regrowth? Biological Conservation, 134: 495-504.

Gaveau, D.L.A., Wich, S., Epting, J., Juhn, D., Kanninen, M. et Leader-Williams, N. 2009b The future of forests and orangutans (Pongo abelii) in Sumatra: predicting impacts of oil palm plantations, road construction, and mechanisms for reducing carbon emissions from deforestation, Environmental Research Letters, vol. 4(3), doi: 10.1088/1748-9326/4/3/034013.

Geist, H.J. et Lambin, D.F. 2002 Proximate causes and underlying driving forces of tropical deforestation. BioScience, 52(2): 143-150.

Glewwe, P., Kremer, M., Moulin, S. et Zitzewitz, E. 2004 Retrospective vs. prospective analyses of school inputs: the case of flip charts in Kenya. Journal of Development Economics, 74(1): 251-268.

Global Observation for Forest and Land Cover Dynamics (GOFC-GOLD) 2009 A sourcebook of methods and procedures for monitoring and reporting anthropogenic greenhouse gas emissions and removals caused by deforestation, 
gains and losses of carbon stocks in forests remaining forests and forestation. http:// www.gofc-gold.uni-jena.de/redd/ (consulté en novembre 2010).

Global Witness 2005 Timber, Taylor, soldier spy: how Liberia's uncontrolled resource exploitation, Charles Taylor's manipulation and the rerecruitment of ex-combatants are threatening regional peace. Rapport de Global Witness soumis au Conseil de sécurité des Nations Unies.

Global Witness 2007 Cambodia's family trees: illegal logging and the stripping of public assets by Cambodia's elite. Global Witness, Londres.

Greene, J.C. et Caracelli, V.J. (éd.) 1997 Advances in mixed method evaluation: the challenges and benefits of integrating diverse paradigms. New Directions in Evaluation, $\mathrm{n}^{\circ} 74$. Jossey Bass, San Francisco, Californie.

Griscom, B., Shoch, D., Stanley, B., Cortez, R. et Virgillo, N. 2009 Sensitivity of amounts and distribution of tropical forest carbon credits depending on baseline rules. Environmental Science and Policy, 12(7): 897-911.

Hamilton, K., Sjardin, M., Peters-Stanley, M. et Marcello, T. 2010 Building bridges: state of the voluntary carbon markets 2010. Rapport préparé par Ecosystem Marketplace et Bloomberg New Energy Finance. http://moderncms. ecosystemmarketplace.com/repository/ moderncms_documents/vcarbon_2010.2.pdf (consulté en novembre 2010).

Hansen, C.P. et Treue, T. 2008 Assessing illegal logging in Ghana. International Forestry Review, 10(4): 573-590.

Hansen, M.C., Roy, D.P., Lindquist, E., Adusel, B., Justice, C.O. et Altstatt, A. 2008 A method for integrating MODIS and Landsat data for systematic monitoring of forest cover and change in the Congo Basin. Remote Sensing of Environment, 112(5): 2495-2513.

Hertel, T., Rose, S. et Tol, R. 2009 Land use in computable general equilibrium models: an overview. Dans : Hertel, T., Rose, S.et Tol, R. (éd.) Economic analysis of land use in global climate change policy, chapitre 1. Routledge, New York.

Honey-Roses, J., Lopez-Garcia, J., Rendon-Salinas, E., Peralta-Higuera, A. et Galindo-Leal, C. 2009 To pay or not to pay? Monitoring performance and enforcing conditionality when paying for forest conservation in Mexico. Environmental Conservation, 36(2): 120-128.
Institut de la Banque mondiale 2005 Introduction to poverty analysis. Banque mondiale, Washington.

Jagger, P. [sous presse] Environmental income, rural livelihoods and income inequality in western Uganda. Dans : Benin, S. et Benson, T. (éd.). In pursuit of prosperity in rural Uganda: opportunities and constraints. Institut International de Recherche sur les Politiques Alimentaires, Washington, DC.

Jagger, P. 2008 Forest incomes after Uganda's forest sector reform: are the rural poor gaining? Programme du CGIAR à l'échelle du système sur l'action collective et les droits de propriété (CAPRi). Document de travail ${ }^{\circ}$ 92. Institut International de Recherche sur les Politiques Alimentaires, Washington, DC.

Jagger, P. et Angelsen, A. [sous presse] What surveys to undertake? Dans : Angelsen, A., Olsen, C.S., Larsen, H.O., Lund, J.F. et Wunder, S. (éd.) Measuring livelihoods and environmental dependence: methods for research and fieldwork. Earthscan, Londres.

Jagger, P., Atmadja, S., Pattanayak, S.K., Sills, E. et Sunderlin, W.D. 2009 Learning while doing: evaluating impacts of REDD+ projects. Dans : Angelsen, A. (éd.) Realising REDD+: national strategy and policy options, p. 281-292. CIFOR, Bogor, Indonésie.

Jenkins, M. 2008 Who murdered the Virunga gorillas? National Geographic, juillet, p. 34-65.

Jenkins, W. 2010 The Tuskegee syphilis study: implications for current research. Communication lors de l'étude sur la population de Caroline, Université de Caroline du Nord, Chapel Hill, 8 octobre 2010.

Jindal, R. 2010 Livelihood impacts of payments for forestry carbon services: field evidence from Mozambique. Dans : Tacconi, L. Mahanty, S. et Suich, H. (éd.) Livelihoods in the REDD? Payments for environmental services, forest conservation and climate change. Edward Elgar, Cheltenham, Royaume-Uni.

Joppa, L.N. et Pfaff, A. 2009 High and far: biases in the location of protected areas. Public Library of Science ONE, 4(12): e8273.

Jumbe, C. et Angelsen, A. 2006 Do the poor benefit from devolution policies? Evidence from forest co-management in Malawi. Land Economics, 82(4): 562-581.

Kaimowitz, D. 1996 Livestock and deforestation in Central America in the 1980s and 1990s: a policy perspective. CIFOR, Bogor, Indonésie. 
Kanninen, M., Murdiyarso, D., Seymour, F., Angelsen, A., Wunder, S. et German, L. 2007 Do trees grow on money? The implications of deforestation research for policies to promote REDD. CIFOR, Bogor, Indonésie.

Karsenty, A. 2008 The architecture of proposed REDD schemes after Bali: facing critical choices. International Forestry Review, 10(3): 443-457.

Kerr, J. et Pender, J. 2005 Farmers' perceptions of soil erosion and its consequences in India's semiarid tropics. Land Degradation and Development, 16: 257-271.

Khandker, S.R., Koolwal, G.B. et Samad, H.A. 2010 Handbook on impact evaluation: quantitative methods and practices. Banque mondiale, Washington, DC.

Krishna, A. 2005 Stages of Progress field manual: a community based methodology for defining and understanding poverty. Version 2.0. http:// sanford.duke.edu/krishna/SoP.pdf (consulté en novembre 2010).

Kusek, J.Z. et Rist, R.C. 2004 Ten steps to building a results based monitoring and evaluation system. Banque mondiale, Washington, DC.

Laporte, N.T., Stabach, J.A., Grosch, R., Lin, T.S. et Goetz, S.J. 2007 Expansion of industrial logging in Central Africa. Science, 316(5830): 1451.

Lawlor, K. 2009 Addressing the causes of tropical deforestation: lessons learned and the implications for international forest carbon policy. Dans : Olander, L.P., Boyd, W., Lawlor, K., Myers Madeira, E. et Niles, J.O. (éd.) International forest carbon and the climate change challenge: issues and options, p. 43-53. Nicholas Institute for Environmental Policy Solutions, Duke University, Durham, Caroline du Nord, États-Unis.

Leeuw, F. et Vaessen, J. 2009 Impact evaluations and development: NONIE guidance on impact evaluation. Banque mondiale, Washington, DC. http://www.worldbank.org/ieg/nonie/guidance. html (consulté en novembre 2010).

Lele, N., Nagendra, H. et Southworth, J. 2010 Accessibility, demography and protection: drivers of forest stability and change at multiple scales in the Cauvery Basin, India. Remote Sensing, 2: 306-332.

Lerman, R. et Yitzhaki, S. 1985 Income inequality effects by income source: a new approach and application to the United States. Review of Economics and Statistics, 67: 151-156.

Lipsey, M.W. 1993 Theory as method: small theories as treatments. New Directions for Program Evaluation, (57): 5-38.
Lopez-Feldman, A. 2006 Decomposing inequality and obtaining marginal effects. Stata Journal, 6: 106-111.

Lopez-Feldman, A., Mora, J. et Taylor, J.E. 2007 Does natural resource extraction mitigate poverty and inequality? Evidence from rural Mexico and Lacandona Rainforest community. Environment and Development Economics, 12: 251-269.

Madeira, E.M., Sills, E., Brockhaus, M., Verchot, L. et Kanninen, M. 2010 What is a REDD+ pilot? A preliminary typology based on early actions in Indonesia. CIFOR InfoBrief. CIFOR, Bogor, Indonésie.

Margoluis, R., Stem, C., Salafsky, N. et Brown, M. 2009 Design alternatives for evaluating the impact of conservation projects. New Directions for Evaluation, (122): 85-96.

Mathiowetz, N.A. et Duncan, G.J. 1988 Out of work, out of mind: response errors in retrospective reports of unemployment. Journal of Business and Economic Statistics, 6(2): 221-229.

McAlpine, C.A., Etter, A., Fearnside, P.M., Seabrook, L. et Laurance, W.F. 2009 Increasing world consumption of beef as a driver of regional and global change: A call for policy action based on evidence from Queensland (Australia), Colombia and Brazil. Global Environmental Change, 19: 21-33.

McCracken, S., Siqueira, A.D., Moran E.F. et Brondizio, E.S. 2002 Land-use patterns on an agricultural frontier in Brazil: insights and examples from a demographic perspective. Dans : Wood, C.H. et Porro, R. (éd.) Deforestation and land use in the Amazon, p. 162-192. University Press of Florida, Gainesville, Florida, États-Unis.

McGee, R. 2000 Analysis of participatory poverty assessment (PPA) and household survey findings on poverty trends in Uganda. Rapport de mission, 10-18 février 2010. www.eldis.org/vfile/upload/1/ document/0708/DOC8301.pdf (consulté en novembre 2010).

McKenzie, D.J. 2005 Measuring inequality with asset indicators. Journal of Population Economics, 18(2): 229-260.

Mertens, B., Sunderlin, W.D., Ndoye, O. et Lambin, E.F. 2000 Impact of macroeconomic change on deforestation in south Cameroon: integration of household survey and remotely-sensed data. World Development, 28(6): 983-999.

Miguel, E. et Kremer, M. 2004 Worms: identifying impacts on health and education in the presence 
of treatment externalities. Econometrica, 72(1): 159-217.

Moran, E.F., Siqueira, A. et Brondizio, E. 2003

Household demographic structure and its relationship to deforestation in the Amazon basin. People and the Environment, p. 61-89. doi: 10.1007/0-306-48130-8_3.

Morton, D., DeFries, R.S., Shimabukuro, Y.E., Anderson, L.O., Arai, E., Bon Espirito-Santo, F., Freitas, R. et Morisette, J. 2006 Cropland expansion changes deforestation dynamics in the southern Brazilian Amazon, Actes de l'Académie Nationale des Sciences des États-Unis, 103(39): 14637-14641.

Moser, C.O.N. 1998 The asset vulnerability framework: reassessing urban poverty reduction strategies. World Development, 26(1): 1-19.

Motel, P.C., Pirard, R. et Combes, J.-L. 2008 A methodology to estimate impacts of domestic policies on deforestation: Compensated Successful Efforts for 'avoided deforestation'. Ecological Economics, 68(3): 680-691.

Mullan, K., Sills, E. and Bauch, S. 2010 The reliability of retrospective data on asset ownership as a measure of past household wealth. Document de travail, Université de l'état de Caroline du Nord, États-Unis.

Murray, B.C. 2009 Leakage from an avoided deforestation compensation policy: concepts, empirical evidence, and corrective policy options. Dans : Palmer, C. et Engel, S. (éd.). Avoided deforestation: prospects for mitigating climate change, p. 11-38. Routledge, New York.

Narain, U., Gupta, S. et van’t Veld, K. 2005 Poverty and environment: exploring the relationship between household incomes, private assets, and natural assets. Document de travail RFF ${ }^{\circ}$ 05-18. Resources for the Future, Washington, DC.

Nelson, A. et Chomitz, K.M. 2009 Protected area effectiveness in reducing tropical deforestation: a global analysis of the impact of protection status. Banque mondiale, Washington, DC, États-Unis. http://siteresources.worldbank.org/INTOED/ Resources/protected_areas_eb.pdf (consulté en novembre 2010).

Neter, J. et Waksberg, J. 1964 A study of response errors in expenditures data from household interviews. Journal of the American Statistical Association, 59(305): 18-55.

Norway 2010 What does the Norwegian Climate and Forest Initiative finance? http://www.regjeringen.no/en/dep/md/ Selected-topics/climate/the-government-of- norways-international-/what-do-we-finance. html?id=557700 (consulté en novembre 2010).

Oliveira, P.J.C., Asner, G.P., Knapp, D.E., Almeyda, A., Galvan-Gildemeister, R., Keene, S., Raybin, R.F. et Smith, R.C. 2007 Land-use allocation protects the Peruvian Amazon. Science, $317: 1233-1236$.

Ortengren, K. 2004 The logical framework approach: a summary of the theory behind the LFA Method. Organisme suédois de développement international (Swedish International Development Agency), Stockholm, Suède.

Ostrom, E. 2009 A general framework for analyzing sustainability of social-ecological systems. Science, 325: 419-422.

Ostrom, E. et Wertime, M.B. 2000 International forestry resources and institutions research strategy. Dans : Gibson, C., McKean, M. et Ostrom, E. (éd.) People and forests: communities, institutions, and governance, p. 1-28. MIT Press, Cambridge, Massachusetts, États-Unis.

Palmer, C. 2001 The extent and causes of illegal logging: an analysis of a major cause of deforestation in Indonesia. Document de travail du CSERGE. Centre for Social and Economic Research on the Global Environment (CSERGE), Londres.

Parker, C., Mitchell, A., Trivedi, M. et Mardas, N. 2009 The little REDD+ book. $2^{\mathrm{e}}$ éd. Global Canopy Foundation, Oxford, Royaume-Uni. Parker, D.C., Manson, S.M., Janssen, M.A., Hoffmann, M.J. et Deadman, P. 2003 Multi-agent systems for the simulation of land-use and landcover change: a review. Annals of the Association of American Geographers, 93(2): 314-337.

Pattanayak, S.K. 2009 Rough guide to impact evaluation of environmental and development programs. Document de travail SANDEE $\mathrm{n}^{\circ} 20$-09. Réseau sud-asiatique pour l'économie du développement et de l'environnement (South Asian Network for Development and Environmental Economics), Kathmandou, Népal.

Pattanayak, S.K. et Sills, E.O. 2001 Do tropical forests provide natural insurance? The microeconomics of non-timber forest product collection in the Brazilian Amazon. Land Economics, 77(4): 595-612.

Pattanayak, S.K., Wunder, S. et Ferraro, P.J. 2010 Show me the money: do payments supply environmental services in developing countries? Review of Environmental Economics and Policy, 4(2): 254-274. 
Pfaff, A. 1999 What drives deforestation in the Brazilian Amazon? Evidence from satellite and socioeconomic data. Journal of Environmental Economics and Management, 37(1): 26-43.

Pfaff, A., Robalino, J., Sanchez-Azofeifa, G.A., Andam, K.S. et Ferraro, P.J. 2009 Park location affects forest protection: land characteristics cause differences in park impacts across Costa Rica, B.E. Journal of Economic Analysis and Policy, 9(2).

Pfaff, A., Robalino, J.A. et Sanchez-Azofeifa, G.A. 2008 Payments for environmental services: empirical analysis for Costa Rica. Document de travail SAN08-05. Duke University Sanford School of Public Policy. http://www.sanford.duke. edu/research/papers/SAN08-05.pdf (consulté en novembre 2010).

Plantinga, A.J. et Richards, K.R. 2008 International forest carbon sequestration in a post-Kyoto agreement. Projet d'Harvard relatif aux accords internationaux sur le climat. Document de travail 2008-11. Cambridge, Massachusetts, États-Unis.

Plewis, I. 2002 Modeling impact heterogeneity. Journal of the Royal Statistical Society: Series A Statistics in Society, 165(1): 31-38.

Poteete, A.R. et Ostrom, E. 2004 Heterogeneity, group size and collective action: the role of institutions in forest management, Development and Change, 35(3): 435-461.

Potvin, C., Tschakert, P., Lebel, F., Kirby, K., Barrios, H., Bocariza, J., Caisamo, J., Caisamo, L., Cansari, C., Casamá, J., et al. 2007 A participatory approach to the establishment of a baseline scenario for a reforestation Clean Development Mechanism project. Mitigation and Adaptation Strategies for Global Change, 12: 1341-1362.

Pradhan, M. et Rawlings, L.B. 2002 The impact and targeting of social infrastructure investments: lessons from the Nicaraguan social fund. World Bank Economic Review, 16: 275-295.

Pro Poor Center et Davies, R. 2006 The 2006 Basic Necessities Survey (BNS) in Can Loc District, Ha Tinh Province, Vietnam. http://mande.co.uk/ special-issues/the-basic-necessities-survey (consulté en novembre 2010).

Rao, V. 2002 Experiments in 'participatory econometrics': improving the connection between economic analysis and the real world. Economic and Political Weekly, 37(20): 1887-1889, 1891.

Ravallion, M. 2009 Evaluation in the practice of development. Banque mondiale, Washington, DC, États-Unis et Oxford University Press, Oxford, Royaume-Uni.

Reynolds, A.J. 1998 Confirmatory program evaluation: A method for strengthening causal inference. American Journal of Evaluation, 19(2): 203-222.

Richards, K. et Andersson, K. 2001 The leaky sink: persistent obstacles to a forest carbon sequestration program based on individual projects. Climate Policy, 1: 41-54.

Richards, M. et Panfil, S. 2010 Manual for social impact assessment of land-based carbon projects. Version 1.0. Forest Trends, Alliance Climat, Communauté et Biodiversité, Fauna and Flora International and Rainforest Alliance, Washington, DC. http://www.forest-trends.org/documents/files/ doc_2436.pdf (consulté en novembre 2010).

Sahn, D.E. et Stifel, D. 2003 Exploring alternative measures of welfare in the absence of expenditure data. Review of Income and Wealth, 49(4): 463-489.

Salim, A., Colfer, C.J.P. et McDougall, C. 1999 Guide de notation et d'analyse pour l'évaluation du bienêtre social. Manuel de critères et indicateurs $\mathrm{n}^{\circ} 7$. CIFOR, Bogor, Indonésie.

Sayer, J., Campbell, B., Petheram, L., Aldrich, M., Ruiz Perez, M., Endamana, D., Nzooh Dongmo, Z.-L., Defo, L., Mariki. S., Doggart, N. et Burgess, N. 2007 Assessing environment and development outcomes in conservation landscapes. Biodiversity Conservation, 16(9): 2677-2694.

Schlamadinger, B., Ciccarese, L., Dutschke, M., Fearnside, P.M., Brown, S. et Murdiyarso, D. 2005 Should we include avoidance of deforestation in the international response to climate change? Dans : Murdiyarso, D. et H. Herawati, H. (éd.) Carbon forestry: who will benefit? Actes de l'atelier sur la séquestration du carbone et les moyens de subsistance durables, Bogor, Indonésie, 16 au 17 février. CIFOR, Bogor, Indonésie.

Schreckenberg, K., Camargo, I., Withnall, K., Corrigan, C., Franks, P., Roe, D., Scherl, L.M. et Richardson, V. 2010 Social assessment of protected areas: a review of rapid methodologies. Rapport pour l'Initiative d'évaluation sociale des aires protégées (Social Assessment of Protected Areas Initiative). International Institute for Environment and Development, Londres.

Shadish, W.R., Cook, T.D. et Campbell, D.T. 2002 Experimental and quasi-experimental designs for generalized causal inference. $2^{\mathrm{e}}$ ed. Houghton Mifflin, New York.

Siegmund-Schultze, M., Rischkowsky, B., da Veiga, J.B., et King, J.M. 2007 Cattle are cash generating assets for mixed smallholder farms in the Eastern Amazon. Agricultural Systems, 94(3): 738-749.

Sills, E., Madeira, E., Sunderlin W.D. et WertzKanounnikoff, S. 2009 The evolving landscape 
of REDD+ projects. Dans : Angelsen, A. (éd.) Realising REDD+: national strategy and policy options, p. 265-280. CIFOR, Bogor, Indonésie.

Sims, K.R.E. 2010 Conservation and development: evidence from Thai protected areas. Journal of Environmental Economics and Management, 60(2): 94-114.

Skutsch, M.M. et McCall, M.K. 2010 Reassessing REDD: governance, markets and the hype cycle: an editorial comment. Climatic Change, 100: 395-402.

Soares-Filho, B., Moutinho, P. Nepstad, D., Anderson, A., Rodrigues, H., Garcia, R., Dietzsch, L., Merry, F., Bowman, M., Hissa, L. et al. 2010 Role of Brazilian Amazon protected areas in climate change mitigation. Actes de l'Académie Nationale des Sciences des États-Unis, 107(24): 10821-10826.

Somanathan, E., Prabhakar, R. et Mehta, B.S. 2009 Decentralization for cost-effective conservation. Actes de l'Académie Nationale des Sciences des États-Unis, 106(11): 4143-4147.

Springate-Baginski, O. et Wollenberg, E. (éd.) 2010 REDD, forest governance and rural livelihoods: the emerging agenda. CIFOR, Bogor, Indonésie.

Sudman, S. et Bradburn, N.M. 1973 Effects of time and memory factors on response in surveys. Journal of the American Statistical Association, 68(344): 805-815.

Sudman, S., Bradburn, N.M. et Schwarz, N. 1995 Thinking about answers: the application of cognitive processes to survey methodology. Jossey-Bass, San Francisco, Californie, États-Unis.

Sunderlin, W.D. et Atmadja, S. 2009 Is REDD+ an idea whose time has come, or gone? Dans : Angelsen, A. et al. (éd.) Realising REDD+: national strategy and policy options. CIFOR, Bogor, Indonésie.

Sunderlin, W.D. et Resosudarmo, I.A.P. 1996 Rates and causes of deforestation in Indonesia: towards a resolution of the ambiguities. Document occasionnel $n^{\circ}$ 9. CIFOR, Bogor, Indonésie.

Sunderlin, W.D., Angelsen, A., Resosudarmo, D.P., Dermawan, A. et Rianto, E. 2001 Economic crisis, small farmer well-being, and forest cover change in Indonesia. World Development, 29(5): 767-782.

Tacconi, L. 2009 Compensated successful efforts for avoided deforestation vs. compensated reductions. Ecological Economics, 68 (8-9): 2469-2472.

Takasaki, Y., Barham, B.L. et Coomes, O.T. 2000 Rapid rural appraisal in humid tropical forests: an asset possession-based approach and validation methods for wealth assessment among forest peasant households. World Development, 28(11): 1961-1977.

Teobaldelli, M., Doswald, N. et Dickson, B. 2010 Monitoring for REDD+: carbon stock change and multiple benefits. Multiple Benefits Série $\mathrm{n}^{\circ} 3$. Centre mondial de surveillance de la conservation de la nature du PNUE, Cambridge, Royaume-Uni.

Uchida, E., Rozelle, S. et Xu, J. 2009 Conservation payments, liquidity constraints, and off-farm labor: impact of the grain-for-green program on rural households in China. American Journal of Agricultural Economics, 91(1): 70-86.

Udry, C. 2003 Fieldwork, economic theory and research on institutions in developing countries. www.econ.yale.edu/ cru2//pdf/fieldwork.pdf (consulté en novembre 2010).

Vedeld, P., Angelsen, A., Sjaastad, E. et Berg, G.K. 2004 Counting on the environment: forest incomes and the rural poor. Environmental Economics Series, No. 98. Banque mondiale, Washington, DC. http://www-wds.worldbank. org/external/default/WDSContentServer/WDSP/ IB/2004/09/30/000090341_20040930105923/ Rendered/PDF/300260PAPER0Counting0on0EN V0EDP0198.pdf (consulté en novembre 2010).

Veldkamp, A. et Lambin, E.F. 2001 Editorial: predicting land-use change. Agriculture, Ecosystems and Environment, 85: 1-6.

Veldkamp, A. et Verburg, P.H. 2004 Editorial: modeling land use change and environmental impact. Journal of Environmental Management, 72: 1-3.

Voluntary Carbon Standard (VCS). 2010 http:// www.v-c-s.org (consulté en novembre 2010).

Walsh, S.J., Crawford, T.W., Crews-Meyer, K.A. and Welsh, W.F. 2001 A multiscale analysis of land use land cover change and NDVI variation in Nang Rong district, northeast Thailand. Agriculture, Ecosystems and Environment, 85: 47-64.

Weber, J., Sills, E.O., Bauch, S. et Pattanayak, S. [sous presse] Do ICDPs work? An empirical evaluation of forest-based microenterprises in the Brazilian Amazon. Land Economics, 87(4): 661-681.

Wertz-Kanounnikoff, S. et Kongphan-apirak, M. 2009 Emerging REDD+: a preliminary survey of demonstration and readiness activities. Document de travail $n^{\circ} 46$. CIFOR. Bogor, Indonésie.

White, H. 2009 Theory-based impact evaluation: principles and practice. Document de travail $\mathrm{n}^{\circ} 3$. 
Initiative internationale pour l'évaluation d'impact (International Initiative for Impact Evaluation), New Delhi, Inde.

Wildlife Conservation Society (WCS). 2006

Household surveys: a tool for conservation design, action and monitoring. Manuel technique $\mathrm{n}^{\circ} 4$. Programme des paysages vivants de la Wildlife Conservation Society, Bronx, NY, États-Unis. http://wcslivinglandscapes.com/landscapes/90119/ bulletins/manuals.html (consulté en novembre 2010).

Wildlife Conservation Society [sans date] Assessing the impact of conservation and development on rural livelihoods: using a modified basic necessities survey in experimental and control communities. Programme des paysages vivants de la Wildlife Conservation Society, Bronx, New York.

Wilkie, D., Morell, G.A., Demmer, J., Starkey, M., Telfer, P. et Steil, M. 2006 Parks and people: assessing the human welfare impacts of establishing protected areas for biodiversity conservation. Conservation Biology, 20(1): 247-249.

Wooldridge, J.M. 2002 Econometric analysis of cross section and panel data. MIT Press, Cambridge, MA, États-Unis.

Wunder, S. 2008 How do we deal with leakage? Dans : Angelsen, A. (éd.) Moving ahead with REDD: issues, options and implications, p. 65-76. CIFOR, Bogor, Indonésie.

Zhang, Q., Devers, D., Desch, A., Justice, C.O. et Townshend, J. 2005 Mapping tropical deforestation in Central Africa. Environmental Monitoring and Assessment, 101(1-3): 69-83.

Zhao, Y. 2003 The role of migrant networks in labor migration: the case of China. Contemporary Economic Policy, 21(4): 500-511. 


\section{Glossaire}

\begin{abstract}
Ajustement sur des covariables : Il s'agit de faire correspondre les unités de contrôle et les unités traitées en fonction de la « distance » séparant les variables pouvant affecter le ou les critères d'évaluation considérés, et donc être source de confusion (covariables). La " distance » est la moyenne de toutes les covariables, pondérée en fonction de l'inverse de la variance.
\end{abstract}

\section{Analyse de régression multiple : Technique statistique consistant à analyser simultanément les relations existant entre une variable dépendante et de multiples variables indépendantes (ou "variables prédictives ou explicatives ») en estimant la valeur de la variation de la variable dépendante en fonction de la variation de chacune des variables indépendantes, les autres variables indépendantes restant constantes. Cette analyse permet d'identifier le caractère significatif des variables indépendantes (à savoir la proportion de changement de la variable dépendante qui peut leur être imputée) ainsi que l'ampleur de leurs effets.}

Attribution : Détermination de la (ou des) cause(s) des résultats observés par l'élimination des explications concurrentes.

Attrition : Sortie de l'échantillon d'étude d'une personne, d'un ménage, d'un site ou de toute autre unité analysée en raison d'un changement de situation ou d'éligibilité, d'une migration, d'une localisation impossible, d'un départ volontaire ou pour toute autre raison.
Biais de sélection : Caractéristique du groupe de traitement ou de l'intervention qui fait que les membres de ce groupe sont plus susceptibles de participer ou de répondre à l'intervention d'une manière donnée et qu'ils présentent des différences systématiques par rapport au groupe de contrôle et à la population générale.

Bien-être économique : Condition humaine, généralement mesurée par des critères économiques.

Bien-être : Condition humaine pouvant être mesurée par des critères économiques, mais également susceptible d'englober des aspects plus généraux liés au bien-être physique et psychologique, tels que l'accès à l'éducation, aux soins de santé et à d'autres services, la participation et le contrôle exercé par les personnes sur les décisions influant sur leur vie, ainsi que les risques et les opportunités qu'ils rencontrent.

Cadre d'échantillonnage : L'ensemble d'unités proprement dit dont a été tiré l'échantillon.

Co-bénéfices : Bénéfices découlant de la REDD+ en plus des bénéfices liés à l'atténuation des changements climatiques, à savoir préservation de la biodiversité, renforcement de l'adaptation aux changements climatiques, soulagement de la pauvreté, renforcement des moyens de subsistance sur le plan local, amélioration de la gouvernance des forêts et protection des droits. 
Coefficient de Gini : Mesure l'inégalité d'une répartition.

Consommation : Valeur des biens achetés et/ou consommés par un ménage.

Déforestation : Conversion à longue échéance ou de façon permanente de terres forestières en terres non forestières. Selon la CCNUCC, la « forêt » est définie comme une zone présentant un couvert vertical au sol minimum compris entre 10 et $30 \%$.

Dégradation : Changements entraînant une diminution de la densité de la forêt ou une perte de carbone forestier, mais ne conduisant pas à la conversion de la forêt en terres non forestières.

Données de panel : Observations faites sur une unité strictement identique (sur le même individu ou le même ménage p. ex.) à des moments très différents.

Données et analyse rétrospectives : Utilisation exclusive de données disponibles actuellement, afin de reconstituer les conditions de l' " avant " (avant l'intervention) pour effectuer une comparaison entre « l'avant » et « l'après ». Les données rétrospectives proviennent d'images de télédétection et d'archives de l'administration ; elles peuvent également être collectées auprès d'experts et de participants de l'étude interrogés sur les conditions antérieures au projet et leurs opinions sur les facteurs, y compris l'intervention, pouvant être à l'origine de l'évolution ressentie de la situation.

Effet moyen du traitement (ATE) : L'effet moyen du traitement sur la population ou les sites considérés, calculé en soustrayant l'effet moyen dans le groupe de contrôle ou groupe témoin de l'effet moyen dans le groupe de traitement ou groupe expérimental si, et seulement si, toute personne/tout site dans la population générale a la même chance de figurer dans le groupe de traitement. En raison de cette hypothèse de randomisation du traitement, le calcul de l'ATE doit être effectué selon une méthodologie de recherche expérimentale.

Effet moyen du traitement sur les traités (ATT) : L'effet moyen d'un traitement sur la population ou les sites considérés, dans le cas où cette population ou ces sites ont reçu le traitement. Calculé en retranchant l'effet moyen dans le groupe de contrôle à l'effet moyen dans le groupe de traitement. Également appelé TOT.

Évaluation d'impact expérimentale : Cf. « Randomisation ».

\section{Évaluation d'impact quasi-expérimentale :} Méthodes utilisant les informations relatives au groupe de traitement pour sélectionner ou constituer statistiquement des groupes de contrôle. Il s'agit des méthodes d' " avant-après/contrôleintervention » (BACI), de l'appariement par le score de propension et de l'ajustement sur des covariables, du plan expérimental de discontinuité de la régression et des variables instrumentales.

Évaluation d'impact : Ensemble défini de plans et de méthodes de recherche visant à analyser et à comprendre l'impact de politiques publiques, de programmes ou de projets, cherchant notamment à déterminer dans quelle mesure les effets mesurés (recherchés ou non) peuvent être attribués à l'intervention considérée et non pas à d'autres causes (Khandker et al. 2010). Cet ensemble de méthodes comprend des techniques expérimentales et quasi-expérimentales. Également appelée «évaluation de programme».

Explications concurrentes : Autres explications pouvant justifier les changements observés des variables d'évaluation, outre l'intervention ( « le traitement ») étudiée. Les techniques d'évaluation d'impact utilisent des méthodes permettant d'éliminer les explications concurrentes de manière fiable, et y compris pour certaines, les explications aussi bien observables que non observables.

Fuite : Réduction d'émissions dues à la déforestation/dégradation obtenue par un projet ou un programme, qui se trouve annulée du fait du déplacement des activités conduisant à des pertes forestières dans un autre endroit situé hors du cadre du projet ou du programme.

\section{Groupe de contrôle ou groupe témoin :}

Population ou site non affecté par le traitement ou l'intervention. 
Hétérogénéité d'impact : Différences d'impact selon la sous-population considérée.

\section{Mécanisme de réponse aux doléances :}

Institution mise en place dans le but de répondre aux préoccupations des personnes et des populations concernées par un projet, un programme ou une politique de conservation ou de développement donnés.

\section{Niveau de référence ou Niveau d'émission} de référence : (1) En évaluation d'impact et dans de nombreux autres domaines, le « niveau de référence » désigne les conditions initiales antérieures au projet. (2) Dans le cadre de la REDD+, les expressions "niveau de référence » et « niveau d'émission de référence » s'emploient indifféremment pour désigner la quantité estimée d'émissions dues à la déforestation ou à la dégradation qui se seraient produites en l'absence de la REDD+ (Angelsen 2008a). (3) Angelsen et al. (2009) attirent l'attention sur la distinction de fond qu'il est essentiel de faire entre les niveaux de référence du scénario de la poursuite de la tendance actuelle (business as usual ou BAU) et les niveaux de référence de comptabilisation. Ces derniers correspondent au taux de perte du couvert forestier devant être amélioré pour démontrer la réalité des réductions et recevoir des paiements, taux sur lequel doivent s'accorder les parties et qui peut être différent des niveaux de référence BAU indiqués par les scientifiques. Le terme niveau de référence est parfois utilisé pour désigner expressément le niveau de référence de comptabilisation.

Perte forestière : S'entend de la déforestation et de la dégradation des forêts.

Porteur de projet : Le porteur de projet REDD+ est la personne ou l'organisation ayant la maitrise et la responsabilité générales de la conception et de la mise en œuvre du projet.

Randomisation : Répartition des participants dans le groupe de contrôle ou dans le groupe de traitement de manière totalement aléatoire, sans tenir compte d'aucun autre facteur (par tirage au sort p. ex.). Lorsque les groupes de contrôle et de traitement sont formés par sélection aléatoire de chaque personne ou site dans la population générale considérée, ces deux groupes devraient être représentatifs de la population générale et posséder des moyennes et des répartitions de caractéristiques équivalentes. Également appelée " évaluation d'impact expérimentale».

REDD+ : Projets, politiques et programmes ayant pour objectif la réduction des émissions dues à la déforestation et à la dégradation des forêts et la préservation, la gestion durable et le renforcement des stocks de carbone forestier. Le « + fait référence à l'élargissement récent de l'objet de comptabilisation et d'incitation aux trois dernières activités.

Retombée : Effet positif ou négatif de l'intervention sur des populations ou des zones non impliquées directement dans l'intervention, ni touchées par celle-ci. Malgré une sélection ou une constitution rigoureuse des groupes de contrôle par le chercheur, le groupe de contrôle peut parfois subir certaines retombées du traitement.

Revenus : Production dont la valeur s'ajoute aux immobilisations corporelles; valeur du total de la production à laquelle on retranche la valeur des acquisitions (mais non la valeur de la main-d'œuvre du ménage ou du capital naturel, tel que la forêt).

Score de propension : Probabilité pour une unité donnée d'être incluse dans le groupe de traitement selon qu'elle possède ou non un ensemble de caractéristiques observées. Permet d'apparier les unités de contrôle et les unités traitées dans la méthode quasi-expérimentale d'appariement par score de propension (ASP).

Situation contrefactuelle : Situation dans laquelle se serait trouvée la population/le site considérés en l'absence d'intervention. Cette situation hypothétique ne pouvant jamais être observée dans les faits, elle doit être estimée par la modélisation, l'observation des variables d'évaluation sur un site de contrôle, la constitution d'un groupe de contrôle par l'emploi de techniques d'évaluation d'impact quasi-expérimentales ou l'association de plusieurs de ces procédés.

Télédétection (des forêts) : Imagerie satellitaire des forêts, permettant de détecter les changements de 
superficie au cours du temps et dans certains cas, les perturbations.

Traitement : Programme, politique, projet ou intervention étudiés.

Validation : Évaluation indépendante par une tierce partie du plan d'un projet conformément à des référentiels définis, notamment pour en établir l'éligibilité par rapport à un référentiel de certification, tel que la Norme de compensation volontaire de carbone (VCS) ou les standards de l'Alliance Climat, Communauté et Biodiversité (CCBA).

Validité externe : Degré auquel les résultats peuvent être généralisés à l'ensemble de la population considérée.

Validité interne : Degré d'exactitude de l'effet de causalité et de l'impact estimés dans l'échantillon d'étude retenu.

\section{Variable confusionnelle (ou facteur de} confusion) : Facteur influant non seulement sur la probabilité de participation ou de réponse à une intervention, mais aussi sur les critères (ou variables) d'évaluation de l'effet de l'intervention considérés. L'influence de ces facteurs doit être prise en compte dans le plan de recherche choisi et au moyen de techniques statistiques de façon à pouvoir évaluer l'impact réel d'une intervention.

Variable explicative : Variable utilisée pour expliquer ou prévoir les changements de valeur de la variable dépendante (ou variable critère). Également appelée variable indépendante.

Variable instrumentale : Variable corrélée à la probabilité de recevoir le traitement, mais qui n'est corrélée avec aucune caractéristique non observée susceptible d'influer sur les critères d'évaluation considérés. Elle peut ainsi servir d' "instrument » du traitement, en permettant de déterminer les impacts par la méthode quasi-expérimentale des variables instrumentales.

Variable de processus : Variable rendant compte d'une caractéristique essentielle de la conception et de la mise en œuvre d'un projet ou d'un programme, ayant une influence sur la manière dont l'intervention conduit aux résultats.

Vérification : Évaluation indépendante par une tierce partie des réductions d'émissions (dans le cas du VCS) ou des bénéfices associés (dans le cas des standards de la CCBA) effectivement apportés par un projet donné concernant le carbone forestier. 


\section{Annexe A. Fiches techniques}

\section{Fiche technique 1 \\ Définition de la terminologie de l'évaluation d'impact et de la REDD+}

Certains lecteurs de ce guide seront sans doute relativement bien informés sur la REDD+, mais ne connaîtront que très peu le domaine de l'évaluation d'impact; d'autres en revanche comprendront bien le concept d'évaluation d'impact sans toutefois être au courant des derniers développements du champ de la REDD+. L'objectif de cette fiche technique est de présenter une définition des termes importants de ces deux domaines qui sont fréquemment utilisés dans ce guide.

Les concepts de la REDD+ et des évaluations d'impact reposent sur un certain nombre de notions clés ; toutefois, ils sont souvent évoqués par une terminologie différente (cf. Tableau 1). Tout d'abord, l'évaluation d'impact a pour objet principal la matérialisation d'un résultat non observable et « contrefactuel ». En d'autres termes, elle modélise ce qui se serait passé pour la zone ou la population visée par une intervention si cette intervention navait pas eu lieu. Le raisonnement contrefactuel est également un critère spécifique de la REDD+. Parce qu'elle est basée sur les résultats, avec octroi de paiements si de nouvelles réductions nettes d'émissions de carbone sont observées, la REDD+ nécessite clairement une prise en compte des scénarios contrefactuels. En règle générale, il s'agit du volume de déboisement (ou de reboisement) qui aurait eu lieu en l'absence de projet ou de programme. Pour la REDD+, ce sont les termes de «Niveau d'émission de référence » (NER) ou « Niveau de référence » qui renvoient à ce concept (Angelsen 2008a). Les concepts de NER et « Niveau de référence " sont très souvent utilisés l'un pour l'autre dans le cadre de la REDD+. Toutefois, Angelsen et al. (2009) attirent l'attention sur la distinction de fond qu'il est essentiel de faire entre les niveaux de référence du scénario de la poursuite de la tendance actuelle (business as usual ou BAU) et les niveaux de référence de comptabilisation. L'estimation des scientifiques du niveau net de déforestation ou de dégradation en l'absence de REDD+ représente le niveau de référence BAU. En revanche, les niveaux de référence de comptabilisation dérivent de négociations politiques : il s'agit du volume de déboisement sur lequel les différentes parties s'accordent et qui représente la limite en-dessous de laquelle les réductions d'émission sont avérées et signifient l'octroi de paiements. Le terme de « Niveau d'émission de référence » désigne parfois spécifiquement le niveau de référence de comptabilisation, qui peut effectivement être semblable le niveau de référence BAU établi par les scientifiques mais qui, en pratique, est fréquemment ajusté par de nombreux facteurs. Dans le cadre de l'évaluation d'impact, l'usage du terme « contrefactuel» est plus proche du terme de

Tableau 1. Des concepts semblables mais une terminologie différente : comparaison des principaux termes utilisés pour décrire l'évaluation d'impact et la REDD+

Concept
Estimation de ce qui aurait eu lieu en l'absence
d'intervention
Effet de l'intervention sur les populations ou les
zones qui ne sont pas directement impliquées ou
concernées par l'intervention
Évaluation d'impact ... Déforestation et REDD + ...

Contrefactuel BAU, Niveau de référence

Retombées Fuite zones qui ne sont pas directem
concernées par l'intervention 
« niveau de référence BAU » pour la REDD+. Le terme " niveau de référence " est également utilisé dans le cadre de l'évaluation d'impact et signifie alors : conditions initiales, antérieures au projet.

Dans les deux domaines, un autre concept intéressant est celui des " retombées ». Une notion sous-jacente importante concernant les méthodes d'évaluation d'impact est que le traitement opéré sur une unité (un village ou un ménage concerné par le projet par ex.) n'influence pas les résultats observés sur les autres unités (villages ou ménages hors du projet par ex.). En réalité, toute intervention en matière de politiques publiques provoque souvent des changements au niveau économique ou environnemental qui à leur tour peuvent avoir des effets bénéfiques ou préjudiciables sur d'autres zones ou populations. Ces effets sont dénommés " retombées », et l'évaluation d'impact est conçue de telle manière qu'elle les exclut (en sélectionnant des contrôles qui ne sont pas affectés par les participants) ou les mesure (par une randomisation à plusieurs niveaux incluant les sous-groupes dont on suppose qu'ils sont affectés par les retombées par ex.). Dans le cadre de la REDD+, ce concept s'inscrit dans le problème de «fuite ». Il faut systématiquement anticiper qu'un certain volume de déboisement/dégradation supprimé par la REDD+ sera tout simplement transféré en dehors des limites du projet/programme.

\section{Terminologie de l'évaluation d'impact}

Attribution : Détermination de la (ou des) cause(s) des résultats observés par l'élimination des explications concurrentes.

Biais de sélection : Caractéristique du groupe de traitement ou d'intervention qui fait que les membres de ce groupe sont plus enclins à participer ou susceptibles de répondre à l'intervention d'une certaine manière et qu'ils présenteront des différences systématiques comparativement au groupe de contrôle et à la population générale.

Cadre d'échantillonnage : L'ensemble d'unités proprement dit dont a été tiré l'échantillon.

Évaluation d'impact expérimentale : Cf. «Randomisation».

Évaluation d'impact quasi-expérimentale : Méthodes utilisant les informations relatives au groupe de traitement pour sélectionner ou constituer statistiquement des groupes de contrôle. Il s'agit des méthodes d' " avant-après/contrôleintervention » (BACI), de l'appariement par le score de propension et de l'ajustement sur des covariables, du plan expérimental de discontinuité de la régression et des variables instrumentales.

Évaluation d'impact : Ensemble défini de plans et de méthodes de recherche visant à analyser et à comprendre l'impact de politiques publiques, de programmes ou de projets, cherchant notamment à déterminer dans quelle mesure les effets mesurés (recherchés ou non) peuvent être attribués à l'intervention considérée et non pas à d'autres causes (Khandker et al. 2010). Cet ensemble de méthodes comprend des techniques expérimentales et quasi-expérimentales. Également appelée " évaluation de programme ".

\section{Groupe de contrôle ou groupe témoin :}

Population ou site non affecté par le traitement ou l'intervention.

Hétérogénéité d'impact : Différences d'impact selon les sous-populations considérées.

Randomisation : Répartition des participants dans le groupe de contrôle ou dans le groupe de traitement de manière totalement aléatoire, sans tenir compte d'aucun autre facteur (par tirage au sort p. ex.). Lorsque les groupes de contrôle et de traitement sont formés par sélection aléatoire de chaque personne ou site dans la population générale considérée, ces deux groupes devraient être représentatifs de la population générale et posséder des moyennes et des répartitions de caractéristiques équivalentes. Également appelée « évaluation d'impact expérimentale ».

Retombée : Effets positifs ou négatifs de l'intervention sur des populations ou des zones non impliquées directement dans l'intervention, ni touchées par celle-ci.

Situation contrefactuelle : Situation dans laquelle se serait trouvée la population/le site considérés en l'absence d'intervention. Cette situation hypothétique ne pouvant jamais être observée dans les faits, elle doit être estimée par la modélisation, l'observation des variables d'évaluation sur un site de contrôle, la constitution d'un groupe de contrôle par l'emploi de techniques d'évaluation d'impact 
quasi-expérimentales ou l'association de plusieurs de ces procédés.

Traitement : Programme, politique, projet ou intervention étudiés.

Validité externe : Degré auquel les résultats peuvent être généralisés à l'ensemble de la population considérée.

Validité interne : Degré d'exactitude de l'effet de causalité et de l'impact estimés dans l'échantillon d'étude retenu.

Variable explicative : Variable utilisée pour expliquer ou prévoir les changements de valeur de la variable dépendante (ou variable critère). Également appelée variable indépendante.

Variable de processus : Variable rendant compte d'une caractéristique essentielle de la conception et de la mise en ouvre d'un projet ou d'un programme, ayant une influence sur la manière dont l'intervention conduit aux résultats.

Variable ou facteur de confusion : Facteur influant non seulement sur la probabilité de participation ou de réponse à l'intervention, mais aussi sur les résultats de l'intervention (mesurés par les critères de jugement). La méthodologie de recherche et les techniques statistiques retenues doivent permettre de neutraliser les effets de ces facteurs, de façon à pouvoir évaluer l'impact réel d'une intervention.

\section{Terminologie de la REDD+ :}

Déforestation : Conversion à longue échéance ou de façon permanente de terres forestières en terres non forestières. Selon la CCNUCC, la « forêt » est définie comme une zone présentant un couvert vertical au sol minimum compris entre 10 et $30 \%$.

Dégradation : Changements entraînant une diminution de la densité de la forêt ou une perte de carbone forestier, mais ne conduisant pas à la conversion de la forêt en terres non forestières.

Fuite : Réduction d'émissions dues à la déforestation/dégradation obtenue par un projet ou un programme, qui se trouve annulée du fait du déplacement des activités conduisant à des pertes forestières vers un autre endroit situé en dehors des limites du projet ou du programme.

\section{Niveau de référence ou Niveau d'émission} de référence : (1) En évaluation d'impact et dans de nombreux autres domaines, le «niveau de référence » désigne les conditions initiales antérieures au projet. (2) Dans le cadre de la REDD+, les expressions "niveau de référence " et « niveau d'émission de référence » s'emploient indifféremment pour désigner la quantité estimée d'émissions dues à la déforestation ou à la dégradation qui se seraient produites en l'absence de la REDD+ (Angelsen 2008a). (3) Angelsen et al. (2009) attirent l'attention sur la distinction de fond qu'il est essentiel de faire entre les niveaux de référence du scénario de la poursuite de la tendance actuelle (business as usual ou BAU) et les niveaux de référence de comptabilisation. Ces derniers correspondent au taux de perte du couvert forestier devant être amélioré pour démontrer la réalité des réductions et recevoir des paiements, taux sur lequel doivent s'accorder les parties et qui peut être différent des niveaux de référence BAU indiqués par les scientifiques. Le terme niveau de référence est parfois utilisé pour désigner expressément le niveau de référence de comptabilisation.

Perte forestière : S’entend de la déforestation et de la dégradation des forêts.

Porteur de projet : Le porteur de projet REDD+ est la personne ou l'organisation ayant la maitrise et la responsabilité générales de la conception et de la mise en œuvre du projet.

REDD+ : Projets, politiques et programmes ayant pour objectif la réduction des émissions dues à la déforestation et à la dégradation des forêts et la préservation, la gestion durable et le renforcement des stocks de carbone forestier. Le « + " fait référence à l'élargissement récent de l'objet de comptabilisation et d'incitation aux trois dernières activités.

Validation : Évaluation indépendante par une tierce partie du plan d'un projet conformément à des référentiels définis, notamment pour en établir l'éligibilité par rapport à un référentiel de certification, tel que la Voluntary Carbon Standard (VCS) ou les standards de l'Alliance Climat, Communauté et Biodiversité (CCBA).

Vérification : Évaluation indépendante par une tierce partie des réductions d'émissions (dans le cas du VCS) ou des bénéfices associés (dans le cas des standards de la CCBA) effectivement apportés par un projet donné concernant le carbone forestier. 


\section{Fiche technique 2}

\section{Mesures des résultats concernant le bien-être économique, physique et psychologique}

\section{Introduction}

Quelle est la meilleure manière de mesurer le bienêtre économique, physique et psychologique? Il s'agit d'une question dont la nature est autant philosophique qu'empirique et pratique. Pour certains, les mesures classiques de bien-être économique semblent les mieux appropriées (à savoir le revenu ou la consommation); pour d'autres, la mesure du bonheur, du niveau de santé ou de la capacité à exercer ses droits sont d'une même importance. La nature des indicateurs de bien-être physique et psychologique qui peuvent être mesurés de manière fiable fait aussi l'objet de nombreux débats. En raison de la divergence des points de vue, de nombreux outils et méthodes d'évaluation et de d'étude des changements de la condition humaine ont vu le jour. Ces différents outils et méthodes s'inscrivent dans 5 catégories :

1. mesure du revenu, soit en tant que production dont la valeur s'ajoute à celles des immobilisations corporelles des ménages ou en tant que consommation, en utilisant des définitions qui sont comparables sur tous les sites ;

2. comptabilité du capital ou des actifs, en utilisant des indicateurs qui peuvent s'avérer subjectifs ou définis d'un point de vue local ;

3. mesure du bien-être physique (santé et état nutritionnel);

4. mesure des perceptions de bien-être et de l'évolution de ce bien-être (bonheur par ex.) ;

5. avec des indicateurs relatifs aux droits, à la sécurité/vulnérabilité des moyens de subsistance et aux opportunités qui s'offrent.

Chaque approche présente ses points forts et ses faiblesses. Celles qui ont recours à plusieurs méthodes peuvent permettre de minimiser l'impact de ces faiblesses et produire une caractérisation plus globale du bien-être. De manière idéale, les mesures quantitatives normalisées ( 1 ou 3 ) devraient se combiner à une méthode rendant compte des perceptions propres à chacun en matière de bien-être physique et psychologique (2, 4 ou 5). L'Annexe B présente plusieurs outils et méthodes fréquemment utilisés dans la mesure du bien-être économique, physique et psychologique.

\section{Mesure de la valeur ajoutée aux immobilisations corporelles des ménages}

Dans les zones rurales des pays en développement, le revenu est en général mesuré comme une valeur qui s'ajoute aux immobilisations corporelles; valeur du total de la production à laquelle on retranche la valeur des intrants achetés (mais non la valeur de la main-d'œuvre du ménage ou du capital naturel, tel que les terres ou la forêt). Le revenu peut-être soit investi/épargné ou utilisé pour la consommation. Les économistes en développement mesurent souvent le bien-être économique à l'aide de données relatives à la consommation. Létude de la consommation de la population peut s'avérer plus facile à effectuer que celle du revenu lorsqu'il y a un niveau élevé de participation dans des activités de subsistance (Sahn et Stifel 2002). Le lissage de la consommation (à savoir l'équilibrage des dépenses et de l'épargne pour obtenir et maintenir le niveau de vie le plus élevé possible) signifie que la variabilité des données relatives à la consommation est moindre que celle des données sur le revenu. De plus, les personnes interrogées trouvent souvent plus simple et plus facile de se souvenir et de mentionner des informations concernant leur consommation que celles relatives à leur revenu, particulièrement si les règlements en liquide représentent une proportion importante des paiements de consommation.

En raison du fait que les interventions de la REDD+ sont susceptibles d'affecter l'accès aux ressources forestières, ce qui altère ensuite la dépendance vis-à-vis de la forêt, une attention particulière doit être portée au choix de méthodes les plus fiables permettant de percevoir l'impact de la modification de l'accès à la forêt sur le bien-être économique 
de ses usagers. Du fait de la nature saisonnière de la consommation et de la commercialisation de la plupart des produits forestiers, ainsi que de leur importance sur le plan de la subsistance (à savoir la consommation directe), les données relatives au revenu annuel total (subsistance et apports) et à la consommation totale (subsistance et dépenses) représentent de manière plus complète les moyens d'existence en zone rurale (Vedeld et al. 2004). Le questionnaire destiné aux ménages de l'étude GCS-REDD+ comprend des questions permettant de préciser les données dont découle la comptabilité du revenu total (Encadré 1).

La représentation détaillée du bien-être économique procurée par les mesures relatives au revenu et à la consommation est un des nombreux avantages de cette méthode. Cette représentation s'avère parfois nécessaire pour détecter la variance de la distribution du bien-être économique entre les sous-groupes (à savoir qui sont les perdants et qui sont les gagnants). De plus, ces mesures étant normalisées elles peuvent être utilisées pour comparer les impacts entre différents sites. Leur normalisation et leur caractère objectif ainsi que le but de leur traitement pour obtenir une comptabilité détaillée du bien-être économique des ménages signifie également que ces mesures peuvent rendre compte d'effets involontaires ou

\section{Encadré 1. Revenu des ménages dans le cadre de l'étude GCS-REDD+ du CIFOR}

L'étude GCS-REDD+ du CIFOR recueille des données à l'aide d'une approche de comptabilité basée sur le revenu total. Ces données relatives à la composition du revenu total des ménages permettent de caractériser l'impact direct des interventions REDD+ sur le revenu total, ainsi que sur le revenu absolu et relatif provenant de la forêt (à savoir la dépendance vis-à-vis de celle-ci). Les variations saisonnières des sources de revenu ont été prises en compte dans la conception du questionnaire destiné aux ménages ; il comporte des périodes variables de remémoration par exemple. Consulter la Section 8 des recommandations techniques de l'étude du GCS-REDD+ et la Section 3 du questionnaire GCS-REDD+ destiné aux ménages. inattendus des projets de la REDD+, comme par exemple la perte de certaines sources de revenu ou un déclin général du bien-être économique.

Cependant, il existe un certain nombre de difficultés associées au recueil de données relatives au revenu et à la consommation annuels des ménages sur une période prolongée. Entre autres les problèmes de souvenirs dus à la longueur des périodes de remémoration (Cavendish 2002) (consulter la Fiche technique 6 qui aborde les méthodes utilisant des données de remémoration qui permettent d'établir la nature des conditions " antérieures »). La période de remémoration pendant laquelle il est possible de mentionner de manière précise la quantité de produits consommés ou ramassés régulièrement peut-être aussi courte que 48 heures (Wilkie, communication personnelle). Une autre difficulté se présente, il s'agit de la nature délicate des informations concernant l'exploitation des produits forestiers; en règle générale une grande partie de ces produits est prélevée illégalement, et les personnes interrogées sont alors prudentes de ne pas révéler trop d'informations sur le revenu et la consommation de produits tirés de la forêt. De plus, la consolidation des données concernant la valeur totale du revenu ou de la consommation nécessite l'utilisation de prix (valeur pondérée) pour tous les produits : ceci peut s'avérer difficile pour les produits de subsistance qui sont consommés directement par le ménage. Finalement, le recueil et le traitement des données du revenu et de la consommation des ménages prennent beaucoup de temps et requièrent des évaluateurs possédant des compétences élémentaires dans le domaine de l'agrégation et de la consolidation de données quantitatives.

\section{Approches basées sur les actifs}

Pour de nombreuses raisons, la détention d'actifs est souvent utilisée pour évaluer le bien-être économique ou la précarité des ménages dans les pays en développement. Tout d'abord, les actifs ne sont pas sujets aux fluctuations à court terme observées pour le revenu et la consommation. Ils permettent donc d'obtenir des informations sur la structure des niveaux de revenu des ménages et le bien-être économique qui en découle (Cohen et Barnes 1996, Carter et May 2001, Filmer et Pritchett 
2001). Dans un second temps, ces données sont plus faciles à mesurer que les autres indicateurs comme le revenu des ménages, les bénéfices de l'agriculture ou les dépenses de consommation (McKenzie 2005, $\mathrm{Vu}$ et al. 2010). Le recueil des données relatives au revenu et à la consommation prend beaucoup de temps et les erreurs de mesure sont parfois considérables (Sahn et Stifel 2003). Non seulement la détention d'actifs contribue directement au bienêtre des ménages mais elle fournit également une indication de leur niveau de vulnérabilité (Moser 1998) et de leur capacité à sortir de la précarité (Sahn et Stifel 2003). La détention d'actifs de production détermine les stratégies de création de revenu qui s'offrent aux ménages (Adato $e t$ al. 2006, Carter et Barrett 2006), alors que des actifs tels que le bétail permettent un lissage de la consommation lorsque les marchés de crédit sont incomplets (Siegmund-Schultze et al. 2007). En conséquence, la situation actuelle d'un ménage est parfois étroitement liée à sa richesse passée (Barrett et al. 2006). La possibilité de caractériser d'un point de vue local les populations relativement riches et celles relativement pauvres est un autre avantage qu'offre l'examen des éventails d'actifs. Par exemple, l'étude GCS-REDD+ du CIFOR développe une échelle de valeur spécifique à chaque village des matériaux utilisés pour la construction des maisons (consulter la Section 4 des recommandations techniques de l'étude GCS-REDD+). En élargissant le champ d'application de ce concept, l'Enquête sur les besoins fondamentaux (Basic Necessities Survey) utilise des méthodes participatives pour établir une liste d'actifs dont « chacun devrait pouvoir disposer et qui ne devraient manquer à personne " (Davies 1997). Ces besoins fondamentaux pourraient comprendre des actifs tels qu'une bicyclette, un quart d'hectare de terres d'agriculture, trois repas par jour et l'accès à la scolarisation. Cette liste est propre à chaque communauté. La normalisation des indicateurs d'actifs permet d'établir des comparaisons entre les différentes communautés.

L'utilisation de listes d'actifs et de mesures du bien-être définis d'un point de vue local fait face à des limitations. Par exemple, si une intervention suscite les attentes de la population sur ce que doit posséder un ménage ou modifie le concept d'un ménage en situation de relative précarité, cette situation risque de compliquer la comparaison des périodes antérieures et postérieures à l'intervention. Même en excluant l'intervention du projet, l'évolution de la technologie et des conditions socioéconomiques peuvent certainement provoquer des changements dans la nature des actifs locaux concernés. L'étude GCS-REDD+ tente d'éviter ce problème en posant des questions sur une liste considérable d'actifs (consulter la Section 2 du questionnaire destiné aux ménages). De plus, les mesures basées sur les actifs négligent parfois de prendre en compte des sources essentielles de revenu ou de consommation cruciales à la compréhension de l'effet des interventions de la REDD+ sur le bien-être : l'importance relative des produits tirés de la forêt sur les moyens d'existence en zone rurale par exemple. Finalement, les actifs peuvent se modifier petit à petit, par rapport au revenu et à la consommation : ainsi, l'impact à moyen terme des projets pourraient ne pas être mesuré très précisément.

\section{Approches basées sur le bien-être physique}

Certaines approches d'évaluation du bien-être mettent l'accent sur l'importance du bien-être physique. Un bon état de santé est une mesure fiable de la qualité de la vie, et certaines mesures de l'état de santé sont de solides indicateurs prévisionnels du développement économique (il existe une forte corrélation entre la mortalité infantile et le PIB par ex.). Un grand nombre d'indicateurs bien connus de bien-être économique, physique et psychologique utilise des mesures de santé. Par exemple, l'Indice du développement humain (IDH) du Programme des Nations Unies pour le développement (PNUD) tient compte de la santé (ainsi que de l'alphabétisation, du taux de scolarisation et du PIB par habitant en valeur de parité de pouvoir d'achat) en mesurant l'espérance de vie à la naissance. De la même manière, le nouvel Indice de pauvreté multidimensionnelle (créé en 2010 par le PNUD afin de mieux mesurer la pauvreté extrême dans les pays en développement) prend en compte la mortalité infantile et la nutrition (à savoir la présence de malnutrition dans le ménage) en plus des huit autres indicateurs d'éducation et de niveau de vie (Alkire et Santos 2010). La malnutrition est évaluée grâce à plusieurs mesures anthropométriques dont la comparaison 
de l'indice de masse corporelle (IMC) des personnes interrogées à la moyenne de leur taille, ou encore par une mesure de la circonférence du milieu du bras qui est ensuite comparée à la moyenne de cette mesure pour des personnes de la même taille. Ces deux approches peuvent être combinées avec des informations sur les maladies déclarées par les personnes (ainsi que la mesure d'actifs et du revenu) pour évaluer le bien-être physique et psychologique des ménages.

\section{Perception du bien-être physique et psychologique ou bonheur}

Les personnes peuvent être directement interrogées sur leur perception du bien-être de leur ménage. Par exemple, le questionnaire GCS-REDD+ comporte les deux questions suivantes : «Au cours des deux dernières années, les revenus de votre ménage étaient-ils suffisants pour couvrir vos besoins? » et « Dans l'ensemble, le bien-être de votre ménage s'est-il amélioré ou dégradé par rapport à la situation dans laquelle vous étiez il y a deux ans? » (consulter la Section 4 du questionnaire GCS-REDD+ destiné aux ménages). Les ménages vivant en zone rurale peuvent être interrogés directement sur ce qui constitue le bien-être physique et psychologique dans leur contexte local et sur la comparaison de leur ménage ou de certains groupes de ménages avec ceux perçus comme étant mieux ou moins bien lotis. Dans ce contexte, le bien-être physique et psychologique est en général envisagé comme l'accumulation de nombreux facteurs comme les attributs financiers, physiques, sociaux, humains et naturels au même titre que le bonheur psychologique en général. Les données concernant les mesures objectives du bonheur comme le nombre de fois où la personne interrogée sourit ou rit pendant l'entretien peuvent aussi servir d'indicateur essentiel du bien-être global.

\section{Approches basées sur les droits, la sécurité/vulnérabilité des moyens de subsistance et les opportunités}

Il existe une autre catégorie d'approches pour mesurer le bien-être qui s'intéressent à la capacité des personnes à exercer leurs droits, à retirer des avantages de toute opportunité ou à s'adapter aux difficultés économiques (ce peut être soit des difficultés de nature covariable qui affectent tous les ménages comme les sécheresses ou les inondations, ou des difficultés idiosyncratiques qui affectent un ménage unique ou un sousgroupe de ménages comme le décès d'un membre âgé de ce ménage ayant une capacité à produire. Ces approches reposent particulièrement sur des méthodes qualitatives et participatives et recueillent de ce fait une grande quantité d'informations sur l'évolution des moyens d'existence et leurs vicissitudes dans une zone spécifique. Par exemple, l'approche de Première évaluation $d u$ bien-être des populations (Basic Assessment for Human Well-being) tente de comprendre si « les parties prenantes concernées jouissent de droits et de moyens reconnus pour gérer les forêts de manière équitable et collaborative " (Colfer et al. 1999). L'accent porté sur la sécurité alimentaire dans certaines approches (CARE 2002 par ex.) s'avère parfois particulièrement adapté dans le contexte des changements climatiques, et facilite l'identification des faiblesses au cours des premières phases du projet pour permettre ainsi d'améliorer la conception des interventions à la fois pour l'atténuation des changements climatiques (à savoir REDD+) et l'adaptation.

\section{Inclusion de services provenant de l'écosystème local dans votre évaluation}

Les forêts intactes donnent accès aux services essentiels de l'écosystème local pour les communautés environnantes en leur procurant des produits de la forêt et de l'eau pure, une protection contre les inondations et les violents orages, et en atténuant la propagation des maladies transmises par des vecteurs. Si ces services écosystémiques représentent des apports importants pour la production, ils doivent être saisis dans la comptabilité du revenu ; s'ils sont importants comme « service» (valeur culturelle), des indicateurs supplémentaires seront requis pour rendre compte des variations de leur disponibilité et leur valeur. Les services écosystémiques fournis localement peuvent parfois représenter des variables intermédiaires importantes affectant la chaîne de causalité reliant une intervention de projet de REDD+ à une variation du bien-être (la théorie du changement par ex.). Pour examiner 
directement les changements de valeur des services écosystémiques, des techniques d'évaluation non marchandes provenant de l'économie environnementale peuvent être utilisées pour convertir ce « revenu » sous forme d'actifs, services et subsistance en une valeur monétaire de manière à ce qu'il puisse être consolidé et comparé aux autres mesures de revenu et de consommation. La prise en compte de tels services et actifs d'origine naturelle à la fois dans le scénario social de référence et les mesures sur le site du projet sera probablement essentielle pour saisir intégralement tous les avantages que les interventions de la REDD+ procurent aux populations locales.

\section{Quel indicateur/méthode de bien- être économique, physique et psychologique choisir?}

Cette fiche de travail présente plusieurs approches couramment utilisées pour recueillir des données relatives aux évolutions du bien-être économique, physique et psychologique. Le Tableau 1 résume les points forts et les faiblesses de chacune d'entre elles. Pour mieux comprendre les effets des interventions de la REDD+ sur le bien-être économique, il est impératif de pouvoir mesurer les changements de la dépendance vis-à-vis de la forêt au cours du temps, et d'utiliser des mesures qui sont identiquement adéquates au cours des périodes antérieure et postérieure à l'intervention.

Tableau 1. Choix d'un indicateur de bien-être économique, physique et psychologique

\begin{tabular}{|c|c|c|c|c|}
\hline & $\begin{array}{l}\text { Mesure de la } \\
\text { valeur ajoutée aux } \\
\text { immobilisations } \\
\text { corporelles des ménages }\end{array}$ & Actifs ou capital & Santé & $\begin{array}{l}\text { Droits, } \\
\text { opportunités et } \\
\text { vulnérabilité }\end{array}$ \\
\hline $\begin{array}{l}\text { Produit des } \\
\text { mesures } \\
\text { directes sur la } \\
\text { dépendance vis- } \\
\text { à-vis de la forêt }\end{array}$ & Oui & $\begin{array}{l}\text { Oui, si elle intègre } \\
\text { l'équipement } \\
\text { d'exploitation } \\
\text { forestière, la } \\
\text { participation à des } \\
\text { groupes d'usagers de la } \\
\text { forêt, etc. }\end{array}$ & Non & Oui \\
\hline $\begin{array}{l}\text { Approprié pour } \\
\text { la mesure des } \\
\text { changements sur } \\
\text { le court terme }\end{array}$ & Oui & Peut-être & Peut-être & Peut-être \\
\hline $\begin{array}{l}\text { Peut utiliser des } \\
\text { mesures définies } \\
\text { localement }\end{array}$ & $\begin{array}{l}\text { Non (sauf pour les valeurs } \\
\text { pondérées ou les prix } \\
\text { locaux des biens de } \\
\text { subsistance) }\end{array}$ & Oui & $\begin{array}{l}\text { Oui, en prenant } \\
\text { en compte le } \\
\text { contexte régional }\end{array}$ & Oui \\
\hline $\begin{array}{l}\text { Exige le recueil } \\
\text { de données } \\
\text { quantitatives }\end{array}$ & Oui & En général & Oui & Non \\
\hline
\end{tabular}




\section{Fiche technique 3}

\section{Méthodologie de recherche expérimentale : randomisation}

\section{Introduction}

Un système de participation volontaire et/ou visant un groupe possédant des caractéristiques précises fait souvent face à un obstacle : le biais de sélection. Ce biais de sélection apparait lorsque le groupe soumis au «traitement» est en essence différent de l'ensemble de la population en termes de caractéristiques qui pourraient avoir une influence sur la manière dont ce groupe répond à l'intervention. Le biais de sélection est un réel obstacle à franchir pour identifier précisément l'impact de bon nombre de politiques publiques (programmes de formation, initiatives de réduction de la pauvreté, paiements pour les programmes des services écosystémiques par ex.), car ces interventions visent par définition certains groupes (personnes sans emploi, en situation précaire, vivant à proximité des zones protégées) ou volontaires. Même les programmes qui ne sont pas spécifiquement ciblés sont affectés par le biais de sélection. Par exemple, les parcs nationaux du monde entier sont en général installés dans des régions reculées loin des voies d'accès ou des marchés (Joppa et Pfaff 2009). La déforestation est moins à craindre dans ces régions reculées qui abritent une population plus pauvre que dans les zones proches des routes: en conséquence, le biais de sélection complique l'identification de l'impact de l'installation des parcs sur les forêts et les populations.

Dans les domaines de la santé et des sciences naturelles, se porter volontaire pour recevoir un traitement ne pose ne général aucune difficulté. Certaines formes de biais de sélection peuvent être évitées en choisissant au mieux une méthodologie de sélection des échantillons. Les chercheurs du domaine médical tentent d'éviter le biais de sélection en effectuant des essais randomisés dans lesquels les volontaires sont affectés soit à un groupe de contrôle ou à un groupe de traitement. Le traitement étant attribué de manière aléatoire, les groupes de traitement et de contrôle doivent être semblables du point de vue de la moyenne et de la distribution des caractéristiques susceptibles d'affecter la manière dont ils répondent à l'intervention. Cette méthode permet d'éliminer le biais de sélection, et diminue considérablement la probabilité que ces caractéristiques affectent le processus d'identification des effets du traitement. La randomisation est plus efficace pour éliminer les biais de sélection que les méthodes quasiexpérimentales présentées dans ce guide (à savoir les méthodes d'appariement ou les méthodes d' " avantaprès/contrôle-intervention » (BACI) associées à l'appariement). En effet, la randomisation élimine les effets des variables de confusion observables (distance par rapport à la route par ex.) et non observables (motivation par ex.). Les méthodes d'appariement, en revanche, vérifient uniquement l'effet des caractéristiques observables. Les méthodes BACI associées à l'appariement permettent également de vérifier les caractéristiques non observables qui demeurent constantes au cours du temps mais pas les caractéristiques non observables qui affectent la sélection ainsi que les résultats et qui évoluent au cours du temps. En imposant des effectifs importants pour les groupes d'échantillons et une méthodologie stricte de randomisation, les impacts peuvent être mesurés directement à partir des différences entre les groupes de traitement et de contrôle. L'estimation de l'impact est beaucoup plus fiable si les études sont effectuées sur des échantillons d'effectifs importants et avec un niveau d'attrition limité ou nul.

Même si l'idée de localiser de manière aléatoire les interventions de REDD+ sur le territoire peut sembler impossible à réaliser voire non souhaitable, cette méthodologie de recherche peut s'appliquer à grand nombre de méthodes et sur des échelles diverses. Dans le domaine de la conservation, les méthodes d'évaluation d'impact randomisées ne sont que très rarement utilisées et il s'avère quelles présentent des difficultés majeures d'ordre financier, politique et pratique (Ferraro 2009). Cependant, la plupart de ces difficultés s'appliquent également aux autres secteurs, et les méthodes de randomisation sont de plus en plus employées pour mieux comprendre les impacts des interventions de développement (consulter les travaux de l'Abdul 
Latif Jameel Poverty Action Lab par ex.). Cette fiche technique propose un aperçu de l'approche par randomisation (ou expérimentale) dans l'évaluation de l'impact et une analyse de nombreuses questions pratiques concernant son utilisation et application dans les interventions de REDD+.

Les méthodologies de recherche randomisées conduisent à des résultats extrêmement fiables tant d'un point de vue interne qu'externe. La validité interne est obtenue en atténuant les effets des variables potentiellement confusionnelles : ceci garantit que les résultats observés dérivent de l'intervention et non de tout autre facteur ou série de facteurs. La validité externe est manifeste lorsque tous les résultats de l'échantillon sont généralisables à l'ensemble de la population représentée par cet échantillon.

\section{Application de la randomisation}

Les avantages évidents de la méthodologie de randomisation (absence de biais de sélection et estimation fiable des impacts) sont toutefois largement dépassés par les difficultés pratiques majeures posées par la mise en œuvre. Le fait qu'un programme puisse être refusé à des populations susceptibles d'en bénéficier pose un problème d'éthique qui préoccupe. L'autre difficulté porte sur la validité externe ou possibilité de généraliser les résultats de l'échantillon à l'ensemble de la population qu'il représente. Certaines personnes se demandent si les résultats des études randomisées nous en apprennent beaucoup sur le monde réel (Ravallion 2009). Une des difficultés majeure est aussi que certaines interventions, telle que la modification d'une politique à l'échelle régionale ou nationale, ne peuvent être randomisées efficacement. Ensuite, les caractéristiques particulières qui affectent la façon dont une population (ou une communauté, forêt etc.) répond à l'intervention ont été annulées par la méthodologie randomisée; pourtant dans le monde réel, les décideurs élaborent leurs politiques en vue de viser ces mêmes caractéristiques. Par exemple, des informations concernant l'impact moyen des interventions de REDD+ sur les forêts d'Indonésie, en général, s'avèrent probablement inutiles pour examiner des politiques car dans la réalité les politiques de la REDD+ visent des zones particulièrement menacées par une conversion (l'Encadré 1 en donne une représentation détaillée).

\section{Encadré 1. Mise en œuvre de méthodologie de randomisation}

\section{Exemple de la REDD+ en Indonésie}

Les projets de REDD+ en Indonésie sont en général localisés dans des zones soumises aux plus fortes pressions de conversion (à savoir densité des routes et de la population), qui présentent les densités de carbone les plus élevées (tourbe) et une valeur de conservation plus importante (parc national). Cette sélection tient aussi compte de la taille du district et du pourcentage de couvert forestier. La validité externe des évaluations de ces projets est donc supposée limitée à des districts de ce type. Un autre facteur crucial pour décider de la zone d'implantation du projet de REDD+ est l'expérience de la région acquise par le porteur du projet, soit directement ou via les activités de conservation de partenaires (Cerbu et al. 2009). De tels facteurs sont délicats à observer et leur examen difficile dans des approches quasiexpérimentales. Dans ce contexte, I'application de la méthodologie de randomisation pour sélectionner la zone du projet pourrait certainement améliorer la validité des résultats de l'évaluation. Par exemple, un programme de subvention pour la REDD+ pourrait permettre d'identifier un groupe de districts éligibles et de sélectionner ainsi de manière aléatoire les districts pouvant recevoir en premier les subventions destinées aux projets de REDD+. D'un point de vue politique, ce système rencontrerait des difficultés, ce qui explique pourquoi les méthodes expérimentales sont rarement employées malgré leur efficacité pour évaluer en toute fiabilité les impacts réels des projets de conservation.

Il est cependant possible de répondre à ces deux préoccupations concernant la randomisation (éthique et importance des résultats dans le cadre des politiques) en utilisant une des stratégies de mise en œuvre suivante.

\section{Mise en œuvre progressive}

Les interventions sont souvent mises en œuvre graduellement en raison des contraintes logistiques ou liées aux ressources. Cet état de fait peut faciliter l'évaluation, si le calendrier de la mise en œuvre dans différentes zones peut être randomisé. Tout 
d'abord, l'ensemble de la zone d'intervention de REDD+ est défini. L'échelle d'une intervention est variable et peut porter sur un groupe de villages dans une zone relativement délimitée ou s'étendre sur une région administrative infranationale. L'intervention de REDD+ est ainsi mise en œuvre par étape et de manière aléatoire dans les villages ou paysages sélectionnés. Le facteur important étant que les sites de première mise en œuvre soient sélectionnés de manière aléatoire : ceci permet d'utiliser comme contrôles les sites qui ne sont pas concernés par l'intervention de REDD+ au départ. Ces sites reçoivent alors également l'intervention de REDD+ plus tard. Il est crucial de tenir compte $\mathrm{du}$ fait que les populations sur ces derniers sites seront conscientes ou non du fait qu'ils peuvent être choisis comme site d'intervention à l'avenir car cette présomption pourrait affecter leur comportement avant l'intervention (conservation de la forêt dans l'espoir de recevoir des subventions de la REDD+ et d'autres incitations par ex.).

Le paragraphe suivant présente le processus de randomisation à deux étapes (Khandker et al. 2010) avec une méthodologie de mise en œuvre progressive.

Étape 1 Définir les caractéristiques des populations ou zones géographiques visées pour la mise en œuvre de projets de REDD+. Il s'agit de la définition de la population générale de laquelle on souhaite déduire des résultats.

Étape 2 Sélectionner de manière aléatoire un sous-échantillon de sites dans cette population générale. Il s'agit de la première étape de randomisation, qui définit le cadre d'échantillonnage et garantit la validité externe des résultats.

Étape 3 Au départ, mettre en œuvre l'intervention de REDD+ dans un échantillon aléatoire parmi les sites sélectionnés. Dans le cadre d'échantillonnage, sélectionner également de manière aléatoire un échantillon de sites qui servent au départ de contrôles. Il s'agit de la deuxième étape de randomisation qui garantit la validité interne des résultats.

Étape 4 Recueillir les données sur les sites de projet et de contrôle (de manière idéale avant et après la mise en œuvre du projet).
Étape 5 Analyser les données à l'aide des tests $t$ de différence de moyennes, des estimations des différences des différences ou d'une analyse de régression.

Étape 6 Identifier les acquis permettant d'améliorer la méthodologie et la mise en œuvre des futurs projets de REDD+.

Étape 7 Mettre en œuvre l'intervention de REDD+ dans les sites qui ont servi de contrôles au départ.

\section{Sur-inscription}

Cette approche (Khandker et al. 2010) est semblable à la mise en ouvre progressive et s'applique également aux situations dans lesquelles les contraintes logistiques ou financières empêchent le porteur du projet de le mettre en œuvre dans tous les sites ciblés; ou encore ne permettent pas l'inscription de tous les ménages qui se portent volontaires. Dans cette méthode, la zone ou population considérée comme réunissant les conditions optimales ou volontaire pour participer à la REDD+ est identifiée en premier, puis un échantillon aléatoire de ce groupe est sélectionné pour la mise en œuvre du projet. Ceux qui ne peuvent recevoir de subvention servent de contrôles. Les contraintes en matière de subventions limitent souvent, si ce n'est toujours, le nombre d'endroits pouvant accueillir un projet de conservation ou de développement ; la méthode de sur-inscription exige de choisir par un échantillonnage aléatoire les endroits ou les populations qui participeront effectivement au projet plutôt que d'utiliser des critères politiques ou autres.

Ces deux méthodologies de randomisation répondent aux préoccupations éthiques suscitées par l'utilisation de sites de contrôle en s'assurant que tous les sites finissent par recevoir une intervention de REDD+ ou en acceptant simplement que, dans les faits, les subventions sont cantonnées à un nombre limité de sites qui peuvent ainsi recevoir ces interventions. Ces méthodologies répondent également aux questions de validité externe et de l'importance pour les politiques de tous les résultats, en définissant clairement au départ les caractéristiques de la zone/population générale où auront certainement lieu les futurs projets de REDD+. 


\section{Fiche technique 4}

\section{Avant-après/contrôle-intervention}

\section{Introduction}

La randomisation est souvent considérée comme l'étalon-or des processus d'évaluation des interventions. Lorsqu'il s'avère impossible de sélectionner aléatoirement les groupes de contrôle et de traitement, la méthodologie avant-après/ contrôle-intervention (BACI) est une approche presque aussi rigoureuse pour évaluer ces relations de causes à effets; ceci, à condition de pouvoir mesurer toutes les variables de confusion susceptibles de varier dans le temps. Dans l'approche BACI, les sites de contrôle doivent être sélectionnés avant la mise en œuvre du projet de REDD+, de manière à ce que les données du niveau de référence soient recueillies au niveau des sites de traitement et de contrôle. En choisissant des sites de contrôle qui sont très similaires à ceux du projet de REDD+, on anticipe que les résultats sur le plan des facteurs sociaux (en moyenne) seraient très semblables dans les deux endroits, si on exclut les effets de l'intervention du projet de REDD+. Les données sur les résultats sont recueillies de nouveau sur les sites de contrôle et d'intervention pendant le déroulement du projet. La différence des changements observée aux sites de traitement et de contrôle est alors utilisée pour calculer l'impact moyen du projet. Ce type d'analyse est dénommé différence de différences.

La mise en œuvre d'une méthodologie de recherche BACI impose la résolution préalable de certains problèmes importants. Tout d'abord, des sites de contrôle adaptés n'existent peut-être pas, ensuite on rencontre souvent une réticence à investir du temps et des ressources pour définir et recueillir des données sur les usagers de la forêt au niveau des sites de contrôle. Si des sites de contrôle presque semblables au site d'intervention de REDD+ (excepté la mise en œuvre de l'intervention de REDD+) peuvent être identifiés, la méthode BACI présente alors un avantage certain : c'est la méthodologie de recherche la plus simple (par rapport à des approches plus complexes comme l'appariement des scores de propension ou la modélisation structurale) qui permet également d'effectuer une analyse simple et transparente. En plus de l'estimation des impacts directs d'un projet, la méthode BACI peut aussi servir pour évaluer les fuites (ou retombées) par des comparaisons de différence de différences avec le site du projet, les sites situés à proximité et supposés être soumis aux fuites et les sites de contrôle (cf. Miguel et Kremer 2002). De même, la méthodologie BACI peut être utilisée pour réaliser une comparaison avec d'autres méthodes de mise en œuvre d'un projet, en recueillant des données sur des souséchantillons d'usagers de la forêt qui participent à ces alternatives en comparant ces sous-échantillons. Enfin, pour mieux comprendre les mécanismes de causes à effets produisant les impacts observés et évalués dans l'étude BACI, l'estimation BACI peut être comparée avec des projections de l'impact ex ante qui peuvent être fondées sur une théorie économique et d'utilisation du sol et/ou sur les opinions des usagers locaux de la forêt (Ravallion 2009, Khandker et al. 2010). Ceci pourrait expliciter la manière dont se produisent les impacts observés et permettre de proposer des moyens d'améliorer les méthodes d'établissement de projections ex ante (pour la validation de projets du marché volontaire du carbone par ex.).

\section{Mise en œuvre de la méthodologie $\mathrm{BACl}$}

\section{Étape 1 : Sélection des sites de contrôles}

De manière idéale, les évaluateurs doivent définir les facteurs susceptibles d'affecter à la fois la participation à l'intervention et également le résultat présentant un intérêt (bien-être économique) : il s'agit en général de caractéristiques biophysiques, d'infrastructure, institutionnelles, socioéconomiques et démographiques. Les données sont recueillies sur un grand nombre de sites de contrôle potentiels; puis, le sous-groupe qui se rapproche le plus des villages de traitement doit être sélectionné en effectuant un " appariement » par rapport à ces variables (consulter la Fiche 
technique 7). En pratique, il se peut que seul un petit nombre de données secondaires soit disponible pour sélectionner les sites de contrôle avant le lancement du projet. Il est important de garder à l'esprit que ces données sont recueillies sur les sites de contrôle pour une raison simple : établir l'attribution, afin d'éliminer toute autre explication contradictoire de la présence des effets observés sur le site du projet, afin d'être sûrs que les modifications observées sur le site du projet sont bien imputables à l'intervention et non à un facteur quelconque. Les évaluations qui sont effectuées sans contrôle engendrent des risques pour la réputation de ces projets car elles pourraient faussement attribuer un déclin du bien-être économique au projet de REDD+ alors qu'il provient en fait d'autres facteurs qui ne dépendent pas du projet (Figure 1).

\section{Étape 2 : Prise en considération des autres variables de confusion potentielles}

Même si les sites de contrôle sont sélectionnés en fonction de la correspondance avec des données secondaires, il peut subsister des différences systématiques à certains égards entre les populations et les conditions sur les sites de contrôle et d'intervention. En conséquence, dans le cadre de l'étude, il est impératif de recueillir des données sur d'autres variables de confusion potentielles qui nétaient pas disponibles dans les sources secondaires. Ces variables comprennent habituellement les caractéristiques du site (caractère saisonnier de l'accès, mesures du capital social) et celles des usagers de la forêt (âge, sexe et origine ethnique des chefs de ménage, nombre d'années de résidence dans cette localité, mesures du capital social). L'appariement en fonction de ces variables de confusion supplémentaires peut être utilisé pour réduire ou limiter la taille des échantillons destinés au recueil de données postérieures à l'intervention,surtout en présence de contraintes de calendrier ou de budget qui limitent le nombre de villages et de ménages pouvant être inclus dans le recueil des données post-intervention.

\section{Étape 3 : Recueil de données avant et après I'intervention de REDD+}

Le recueil de données tant avant qu'après l'intervention de REDD+ et au niveau des sites de contrôle et d'intervention est impératif car il est impossible de trouver deux sites ou deux groupes de personnes identiques à $100 \%$ pour ce
Étape 1

Identification des indicateurs

- Intervention à évaluer

- Indicateurs de processus observables

- Résultats spécifiques étudiés

- Indicateurs de résultats observables

- Identification du groupe de contrôle
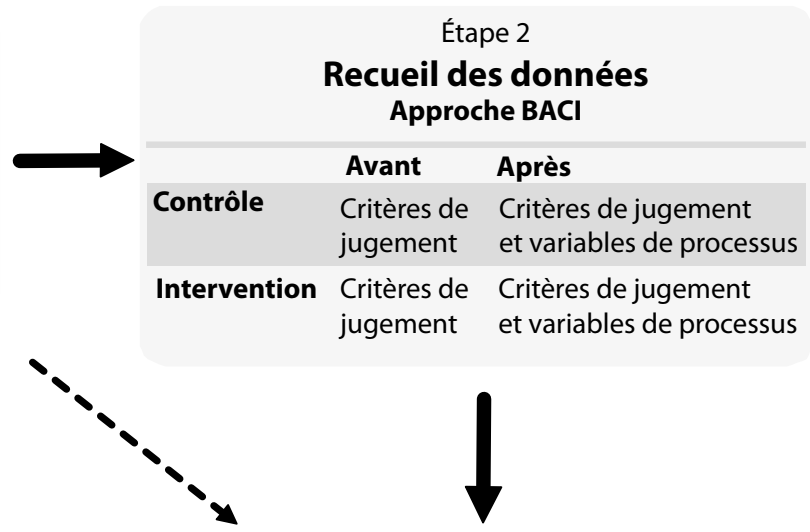

Étape 4

Diffusion des enseignements

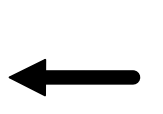

Étape 3

Analyse

Processus - Impacts

Figure 1. Méthodologie et mise en œuvre BACl (adapté de Jagger et al. 2009) 
qui est de leurs caractéristiques observables et non observables. Les caractéristiques non observables comprennent des attributs comme la motivation qui peut certainement affecter la participation au projet ainsi que les résultats; toutefois, les données secondaires qui s'y rapportent n'existent probablement pas ou sont imperceptibles pour le chercheur. Dans la mesure où ces caractéristiques non observables ne varient pas au cours du temps (à savoir qu'elles sont constantes), elles affectent les critères de jugement de manière similaire avant et après l'intervention. Ainsi, la différence entre les critères de jugement au cours du temps peut être comparée entre les sites sans avoir à tenir compte de ces caractéristiques non observées. Au possible, l'examen portera sur les mêmes ménages pour les périodes « avant » et « après », ce qui permettra de créer un ensemble de données de panel au niveau du ménage. Les données de panel contiennent des observations concernant de nombreuses variables observées au cours de plusieurs périodes de temps à partir de la même unité d'observation. Cependant, s'il s'avère impossible d'examiner les mêmes ménages au cours de ces deux périodes de temps, une autre option est envisageable : extraire un nouvel échantillon aléatoire de villages ou de ménages dans la deuxième période de temps afin de créer un ensemble de données regroupées et transversales (Wooldridge 2002).

\section{Analyse}

Les données BACI peuvent être analysées au moyen de plusieurs méthodes de différence de différences. La première étape est souvent une comparaison pour identifier les sous-échantillons de contrôle qui sont les plus comparables aux échantillons de traitement (consulter la Fiche technique 7). Les échantillons appariés sont alors analysés au moyen de simples tests de différence de moyennes ou d'analyses de régression à plusieurs variables, afin de neutraliser les différences dues aux variables de confusion. Si un biais de sélection se manifeste (parce que les variables importantes des sites de contrôle et d'intervention n'ont pas été appariées ou encore que l'appariement effectué ne se soit pas déroulé comme prévu), il est possible d'utiliser la « méthodologie de comparaison avec un groupe témoin non équivalent " pour éliminer les différences systématiques entre les groupes de contrôle et d'intervention (Shadish et al. 2002). En l'absence de données de panel, la méthodologie " différence de différences conditionnelle " permet d'éliminer ces différences systématiques entre les groupes « avant » et " après ». Jagger (2008) utilise ces deux méthodes pour évaluer les impacts de la réforme de décentralisation en Ouganda sur le revenu provenant de la forêt pour différents groupes de revenus. 
Fiche technique 5

\section{Approches modifiées " avant-après " et « contrôle- intervention »}

\section{Introduction}

Lorsqu'il est impossible d'utiliser la randomisation ou la méthode avant-après/contrôle-intervention (BACI), les deux meilleures méthodologies sont une comparaison contrôle-intervention (CI) ou une comparaison avant-après (BA) qui tient compte d'un scénario contrefactuel prévisionnel. Cette fiche technique analyse le principe des deux approches CI et BA modifiées et la manière de les mettre en ouvre.

\section{Surmonter les effets " des analyses contrefactuelles contrefaites $»$ : approches modifiées « avant-après " et « contrôle-intervention "}

La simple comparaison des conditions postérieures au projet aux conditions antérieures ou avec celles se manifestant sur un autre site, pour attribuer ensuite au projet toute différence de résultats observée, ne permet pas d'écarter les autres causes qui pourraient expliquer les résultats observés en matière de bien-être économique. C'est la raison pour laquelle les comparaisons directes avant-après ou avec-sans sont souvent dénommées « analyses contrefactuelles contrefaites ». Cependant, le recueil de données uniquement au niveau du site du projet à deux moments distincts (comme pour BA) ou sur plusieurs sites mais à un moment unique (comme pour CI) présente l'avantage de solliciter moins de ressources que la méthode BACI. Heureusement, il est tout à fait possible d'améliorer l'exactitude des résultats obtenus par ces approches en y ajoutant quelques étapes essentielles. Ces méthodes sont dénommées approches $\mathrm{CI}$ et $\mathrm{BA}$ « modifiées " afin de mettre l'accent sur le fait qu'elles ne sont pas de simples comparaisons des résultats du projet aux conditions antérieures au projet ou à des zones de contrôle non appariées. Ces approches modifiées s'avèrent être des options intéressantes en présence de contraintes budgétaires ou si dans les faits la planification d'une évaluation rigoureuse de l'impact ne peut commencer que bien après le lancement des projets.
La modification de la méthodologie de recherche CI afin de garantir un appariement correct des sites de contrôle et d'intervention peut annuler certaines faiblesses de l'approche CI habituelle. L'appariement des sites de contrôle et d'intervention par rapport aux caractéristiques observables qui conditionnent à la fois la participation à une intervention et les résultats étudiés peut améliorer de manière significative l'exactitude des résultats concernant l'impact évalué. Par exemple, Andam et al. (2008) évaluent l'impact sur la déforestation des zones protégées au Costa Rica et révèlent que si les méthodes d'appariement ne sont pas utilisées, les comparaisons directes contrôle-intervention surestiment de près de $65 \%$ le volume de déboisement évité grâce aux parcs. Cependant, il peut subsister des différences systématiques et non observables, même entre des sites de contrôle et d'intervention correctement appariés, lesquelles sont susceptibles de fausser la détermination de l'impact. La Fiche technique 7 présente des directives concernant les méthodes d'appariement. L'adoption d'un raisonnement contrefactuel et l'élaboration d'une estimation approximative de la situation en l'absence de projet permettent de surmonter certains des problèmes de la méthode de comparaison avant-après. Une telle approche modifiée peut suivre les étapes suivantes.

Étape 1 Recueil de données décrivant les conditions initiales au niveau du site du projet.

Étape 2 Utilisation de ces données " antérieures" et celles provenant d'autres sources pour estimer ce qui se serait passé si le projet n'avait pas eu lieu.

Étape 3 Recueil d'un deuxième ensemble de données « postérieures » au projet.

Étape 4 Comparaison des modifications observées entre les conditions « antérieures » et " postérieures » avec celles anticipées à l'étape 2. 


\section{Encadré 1. Évaluation de l'impact social selon les standards de l'Alliance Climat, Communauté et Biodiversité}

Les standards de I'Alliance Climat, Communauté et Biodiversité (CCBA) exigent que les projets de carbone forestier fassent preuve d'impacts sociaux positifs nets sur les communautés locales. Pour atteindre ces objectifs, les projets doivent :

1. décrire les conditions socioéconomiques de la communauté au moment du lancement du projet ;

2. élaborer un scénario socioéconomique «sans projet »;

3. expliciter la manière dont le projet va améliorer ces conditions socioéconomiques;

4. établir un système de surveillance des impacts sociaux;

5. évaluer les conditions socioéconomiques après le projet.

Jusqu'à une période récente, la CCBA n’a fourni aucune recommandation spécifique aux porteurs de projets sur la manière de mettre en œuvre ces cinq étapes et de fournir des éléments de preuve sur les impacts positifs nets attribuables au projet au moment de la validation du projet. Afin de pallier cette lacune, la CCBA et ses partenaires ont élaboré récemment le Manual for Social Impact Assessment of Land-Based Carbon Projects (Manuel d'évaluation de l'impact social des projets de carbone terrestre, ci-après « le Manuel »). La première version du Manuel (Richards et Panfil 2010) est actuellement testée sur les sites et la version révisée est attendue en 2011.

Le Manuel suggère des méthodes permettant de mettre en évidence la conformité aux standards de la CCB, tout en faisant remarquer qu'il existe une grande variété de méthodes utilisables pour répondre à ces exigences. Si I'on veut que les standards CCB restent accessibles et utilisés à grande échelle, il est indispensable de trouver un équilibre entre les coûts de surveillance et d'évaluation d'une part et la rigueur de la mise en évidence de I'attribution d'autre part ; le Manuel rend compte de méthodes qui permettent de maintenir cet équilibre. II met l'accent sur l'importance de l'élaboration d'une théorie du changement satisfaisante (raisons pour lesquelles un projet peut avoir des impacts positifs et négatifs à la fois), puis de la concentration des efforts de recueil de données sur certains points essentiels de la chaîne de causalité, ceci afin de produire une évaluation d'impact social à moindre coût. Le Manuel admet que le développement d'un scénario social de référence « sans projet » est essentiel pour établir les liens d'attribution et il recommande en général des méthodes participatives qui exigent des différentes parties d'envisager les conditions sociales en l'absence de projet.

Au cours de la deuxième étape, les données contrefactuelles peuvent être estimées ex ante en extrapolant les tendances du passé à des périodes ultérieures ou en réalisant une prévision des tendances d'avenir à l'aide de modélisations statistiques ou à partir d'opinions d'experts locaux (qui peuvent être des usagers des ressources locales). Remarque : l'approche utilisant les prévisions ex ante ressemble fortement aux analyses contrefactuelles concernant le déboisement ou la dégradation (à savoir les "niveaux de référence ») établies dans la REDD+. Cependant, cette approche peut ne pas être assez puissante pour annuler le problème de la validité des hypothèses à la base des données contrefactuelles anticipées. Si ces hypothèses ne sont pas vérifiées, cette approche ne fonctionne pas correctement : la mise à l'épreuve de ces hypothèses devra probablement être effectuée avec des données d'observation provenant d'un site de contrôle ou d'une région de référence. Cependant, si les prévisions ex ante se retrouvent modifiées au cours de la période "postérieure » par des données secondaires appropriées relatives à d'autres origines possibles pour expliquer l'évolution du bien-être économique (dévaluation de la monnaie, sécheresse par ex.), alors l'exactitude de l'évaluation en l'absence de projet peut en être améliorée. Cette approche est également similaire à celle adoptée pour les niveaux de référence de déforestation/dégradation, qui doivent être « rectifiés » à intervalles réguliers pour tenir compte de l'amélioration des modèles et des estimations de carbone. 
L’Alliance Climat, Communauté et Biodiversité (CCBA) développe en ce moment des recommandations pour les porteurs de projets ; elles concernent la mesure des impacts sociaux au moyen d'une approche BA modifiée (Richards et Panfil 2010). Cette approche consiste plus particulièrement à faire une prévision générant des données contrefactuelles ex ante et à recueillir des données « avant » et « après » pour un ensemble d'indicateurs associés au modèle de causes à effets du projet ou à la théorie du changement. L'encadré 1 présente cette approche de manière détaillée. 


\section{Fiche technique 6}

\section{Reconstruction des conditions « avant " avec des données rétrospectives}

\section{Introduction}

Les évaluations sont souvent amorcées longtemps après le lancement d'un projet : l'évaluation et l'attribution des changements sont alors d'autant plus difficiles à évaluer. Pour les nouveaux projets de REDD+, il est probable que les informations relatives aux niveaux de référence seront recueillies sous une forme ou une autre afin de répondre aux exigences de certification; ces projets doivent concevoir l'obtention de ces données et les archiver afin de produire un niveau de référence pour les évaluations ultérieures. Cependant, les porteurs de projets de REDD+ peuvent encore être impliqués dans des évaluations ex post des efforts de conservation de la forêt ayant eu lieu dans le passé et qui ont éprouvé des stratégies potentielles de réduction de la déforestation et de la dégradation.

Lors de l'évaluation d'un projet pour lequel on ne dispose d'aucune donnée relative aux niveaux de référence, la première décision méthodologique à prendre concerne le recueil ou non de données au niveau de sites ou de ménages de contrôle (ciaprès dénommés " unités ») qui ne sont pas affectés par le projet. Après avoir recueilli ces données relatives aux témoins (méthodologie de recherche contrôle-intervention), on emploie souvent un processus d'appariement pour sélectionner et pondérer un échantillon de témoins et le comparer aux unités d'intervention affectées par le projet. Comme le présente la Fiche technique 7, les unités d'intervention et de contrôle doivent être appariées en tenant compte de facteurs dont dépendent à la fois la participation au projet et les résultats étudiés, mais ces facteurs ne doivent pas être influencés par le projet. Il peut s'agir de caractéristiques figées et permanentes (comme la moyenne de la pente de terrain dans la zone d'une communauté ou l'origine ethnique du chef de ménage) ou encore de caractéristiques prédéterminées (comme le couvert forestier d'une communauté ou le niveau de richesse d'un ménage avant le projet) ; ces dernières doivent être reconstruites d'une manière ou d'une autre.
Si le recueil des données sur les unités de contrôle est impossible à réaliser, soit en raison de contraintes budgétaires ou en l'absence d'unités comparables, la dernière méthodologie de recherche dont on dispose est dénommée « rétrospective ». Elle consiste à recueillir des données uniquement «après » l'intervention et seulement au niveau du site "d'intervention", puis à déterminer l'attribution des impacts du projet au moyen de données rétrospectives relatives aux critères de jugement étudiés (la valeur de ces critères avant le projet ou leur variation depuis le lancement du projet) ou en interrogeant directement les personnes pour recueillir leur opinion au sujet des impacts du projet (consulter la méthode de " comparaison autoréférentielle ", (reflexive comparison) de l'attribution dans Schreckenberg et al. 2010).

Les données rétrospectives proviennent de sources définies au sens large comme la télédétection, les statistiques officielles ou l'obtention directe via des instruments de recherche. Chacune de ces approches présente des problèmes inhérents liés à l'échelle ou l'unité d'analyse (les statistiques officielles ne sont peut-être pas disponibles pour les niveaux de la communauté ou du ménage par ex.) et à l'indicateur de résultat (ou critère de jugement) considéré (la remémoration du revenu ou de la consommation est en général plus difficile que celle qui concerne des actifs ponctuels par ex.). Quelle que soit l'origine de ces données rétrospectives, elles posent toutes une question d'ordre méthodologique concernant la période de temps à laquelle elles se rapportent : que doit-on retenir comme période « avant » pour le projet?

\section{Définition des périodes à considérer}

Dans les meilleures conditions, les données rétrospectives permettant d'apparier ou d'estimer les impacts doivent correspondre à la période de temps située immédiatement avant l'annonce du projet ou avant qu'il ne commence à influencer 
les comportements. L'utilisation de données recueillies après le lancement du projet pour établir les niveaux de référence risque de sous-estimer les effets du projet (dans la méthode rétrospective) et de créer un biais lors de la sélection des témoins du groupe de contrôle (dans la méthode contrôleintervention). Inversement, l'utilisation de données recueillies longtemps avant le lancement du projet diminue la précision des estimations mais ne crée pas de biais de sélection. Par ailleurs, comme indiqué ci-après, le renvoi à des événements importants (sécheresse, élection par ex.) peut améliorer l'exactitude des souvenirs, ce qui peut être un élément à prendre en considération dans le choix de la période d'obtention des données rétrospectives.

En plus des données fournies par la télédétection ou les instances officielles, il s'avère très utile de recueillir des données à deux moments antérieurement au projet ainsi qu'au cours d'une troisième observation ultérieure à celui-ci. Ce système inhérent aux méthodologies rétrospectives permet d'examiner l'influence potentielle du projet sur les tendances qui se dégagent au niveau des critères de jugement. Dans la méthodologie contrôle-intervention, la validité du groupe de comparaison peut être évaluée en analysant l'existence de différences significatives pour les valeurs historiques des critères de jugement entre les groupes d'intervention et de contrôle (il s'agit en fait de la réalisation d'un test de falsification, car les valeurs présentes des critères de jugement ne doivent pas être affectées par une assignation ultérieure au traitement).

\section{Données secondaires ou de télédétection}

Les données secondaires et de télédétection sont couramment utilisées pour établir la valeur des critères de jugement « avant » un projet, soit pour effectuer une comparaison directe avec les valeurs de ces critères observées ultérieurement au projet ou pour le processus d'appariement en vue de sélectionner et de pondérer le meilleur groupe de comparaison. Par exemple, Andam et al. (2010), Joppa et Pfaff (2009), Nelson et Chomitz (2009) et Soares-Filho et al. (2010) exploitent des données historiques secondaires et de télédétection pour évaluer les impacts des zones protégées sur le couvert forestier.

L'utilisation de données secondaires pour l'évaluation des projets de REDD+ est souvent restreinte par l'absence de correspondance entre les échelles et les unités : les communautés prises en compte pour le projet ne s'inscrivent peut-être pas parfaitement dans les grilles de recensement ou dans les unités administratives utilisées par les organismes gouvernementaux par exemple. L'utilisation de la télédétection est également entravée par les échelles ainsi que les coûts (d'obtention et d'exploitation des données), la durée de la période (relative au moment où les images de télédétection ont été archivées) et le couvert nuageux. Un autre point important à prendre en considération : l'acquisition d'images par le même instrument de détection associée à des méthodes de classification identiques pour les deux types de données « avant » et " après » améliorent singulièrement la qualité de l'analyse.

\section{Remémoration avec le questionnaire destiné aux ménages}

Demander aux ménages de se souvenir de leur détention d'actifs, de l'utilisation des terres ou des autres activités économiques au cours d'une période antérieure fait partie des pratiques habituelles utilisées dans les études sur la dynamique des exploitations agricoles et des ménages en zone rurale dans les pays en développement (Mertens et al. 2000, Takasaki et al. 2000, Walker et al. 2000, McCracken et al. 2002, Moran et al. 2003 par ex.). Des données rétrospectives ou de remémoration obtenues ainsi ont été utilisées pour évaluer l'impact des crises financières (Sunderlin et al. 2001), des réformes politiques (Pradhan et Rawlings 2002, Uchida et al. 2009), des aires protégées (Schreckenberg et al. 2010) et des événements spécifiques aux ménages comme les migrations (Zhao 2003, Boucher et al. 2005). Cependant, bien qu'elles soient fréquentes, ces " pratiques d'excellence » et la fidélité des souvenirs ont rarement été évaluées dans le contexte des questionnaires destinés aux ménages dans les régions en développement. Il existe toutefois quelques exceptions à la règle : entre autres, l'étude de Beckett et al. (2001) sur l'histoire 
maritale et les antécédents de fertilité des femmes en Malaisie, et celle de Glewwe et al. (2004) sur l'effet du matériel dont disposent des écoles sur les résultats scolaires au Kenya.

Pour utiliser le questionnaire destiné aux ménages, on remémore souvent aux personnes interrogées la période de temps souhaitée en évoquant un événement (catastrophe naturelle, élection, Coupe du monde etc.) qui nest pas en relation directe avec le projet en cours d'évaluation. Cependant, des recherches tendent à montrer que toutes les personnes nordonnent pas leur mémoire en fonction des mêmes types d'information. Ainsi, le type d'événement (ou autre rappel) qui sert le mieux d'ancrage pour exprimer ses souvenirs reste mal connu (Sudman et al. 1995). De même, il nest pas avéré que des périodes de remémoration écourtées génèrent des souvenirs plus exacts. Sudman et Bradburn (1973) signalent que les personnes interrogées ont plus tendance à amplifier les éléments ou événements lorsque la période de remémoration est courte et quelles sont plus à même d'en oublier quand cette période est longue. Mathiowetz et Duncan (1988) ont découvert que la longueur de la période de rémémoration est moins essentielle que l'importance de l'événement (dans le cas décrit ici, la durée de période sans emploi).

Le facteur qui semble systématiquement altérer le souvenir est la taille ou la pertinence de l'événement ou de l'élément dont on doit se souvenir. Par exemple, les valeurs faibles et les actifs habituels (Mullan et al. 2010), les réparations peu chères (Neter et Waksberg 1964) et les maladies bénignes (Bernard et al. 1985) sont le plus souvent oubliés. Dans une comparaison sur une période de neuf ans entre les données de panel et les données rétrospectives relatives aux actifs, Mullan et al. (2010) ont découvert que les ménages les plus pauvres se souviennent en général plus précisément des actifs qu'ils possédaient auparavant, probablement car ces mêmes actifs sont d'autant plus importants quand on est pauvre.

Mullan et al. (2010) ont également remarqué que si on interroge plus d'une personne par ménage, moins d'actifs sont oubliés, toutefois cette méthode conduit aussi à une augmentation du nombre d'actifs dont les personnes se souviennent de manière inexacte (dans les faits : mention d'une détention d'actifs neuf ans auparavant alors que les données actuelles de cette branche du panel indique l'absence de possession d'actifs). Les chercheurs en ont donc conclu que même si les données rétrospectives permettent d'obtenir une mesure approximative du niveau de richesse antérieur des ménages, ces données font l'objet de perturbations d'ampleur significative, particulièrement en ce qui concerne les actifs de moindre valeur et les ménages les plus fortunés.

\section{Autres méthodes}

Les données rétrospectives sont également recueillies en utilisant des méthodes de recherche participatives et/ou qui travaillent avec des groupes. Elles offrent l'avantage d'être plus exactes car elles opèrent une triangulation intrinsèque entre les membres du groupe. Schreckenberg et al. (2010) décrivent deux de ces méthodes dénommées " changement le plus significatif» (Most significant change) et «évaluation participative quantitative " (Quantitative participatory evaluation).

L'«évaluation participative de la pauvreté » (Participatory poverty evaluation) est une troisième approche qui utilise également une technique de remémoration en groupe; en travaillant avec des groupes de consultation pour produire des récits ou un calendrier historique de la collectivité, cette technique permet de générer ensuite une analyse des tendances en fonction du temps, propres à cette communauté ; cette analyse s'appuie sur l'utilisation de matrices de notation (McGee 2000). Ce processus permet d'établir des références croisées entre des personnes et des périodes de temps variables définies par les personnes interrogées. Pourtant, même si cette méthode améliore l'exactitude des résultats, elle convient moins bien à la comparaison de la variation de l'impact des résultats parmi les personnes interrogées, ou encore pour apparier participants et non participants du projet. 
Fiche technique 7

\section{Appariement des sites/ménages des groupes de contrôle et d'intervention}

\section{Introduction}

Les sites de contrôle et d'intervention doivent se ressembler le plus possible. Dans le cas contraire, il est impossible de comparer les résultats, concernant le bien-être économique et l'utilisation des terres, observés au niveau des sites de contrôle et d'intervention puis d'en déduire des conclusions fiables attribuant toute différence entre les deux sites aux effets de l'intervention et non pas à un autre facteur ou ensemble de facteurs. Si les sites sont différents, le biais de sélection peut fausser l'interprétation de l'impact. L'appariement des sites compte tenu de caractéristiques qui peuvent déterminer le placement dans l'intervention/la participation à l'intervention (distance par rapport à la route ou capital social par ex.) ainsi que le résultat étudié (bien-être économique par ex.) est une stratégie efficace pour atténuer les difficultés posées par le biais de sélection. Prêter attention au biais de sélection peut s'avérer particulièrement important dans les interventions axées sur la performance, comme la REDD+. En effet, les sites sélectionnés pour le projet de REDD+, précisément
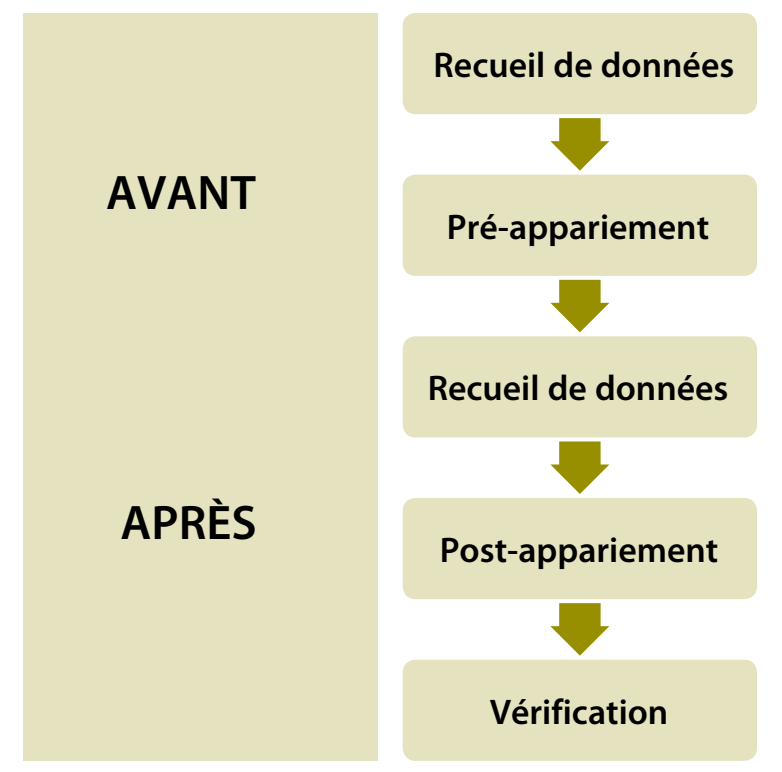

Figure 1. Calendrier des étapes du recueil de données et de l'appariement parce qu'ils sont perçus comme différents, offrent plus de chances de réussite que d'autres. De plus, au sein des projets de REDD+, la probabilité qu'un ménage se porte volontaire pour participer au projet (dans un système octroyant des paiements pour des services écosystémiques (PSE) par ex.) est aussi probablement déterminée en partie par les caractéristiques du ménage qui influencent les résultats concernant l'utilisation des terres et le bien-être économique. Cette fiche technique présente différentes méthodes d'appariement et la façon de les mettre en œuvre. Dans cette fiche, toute référence faite aux « sites » d'appariement s'applique également à tous les niveaux analysés : il peut donc s’agir de villages ou de ménages.

\section{À quel moment effectuer I'appariement}

Les sites peuvent être appariés avant ou après les recherches approfondies sur le terrain (questionnaires destinés aux ménages par ex.). Dans les meilleures conditions, deux appariements sont réalisés : le pré-appariement et le post-appariement (consulter la Figure 1). Ceci revient à :

(1) sélectionner des sites de contrôle qui correspondent le mieux aux sites d'intervention compte tenu des caractéristiques principales (préappariement) ;

(2) mener une activité de recherche au niveau des sites de contrôle et d'intervention avant le début de l'intervention ;

(3) affiner le processus d'appariement en fonction des données recueillies au cours des études sur le terrain (post-appariement) ;

(4) mener des recherches au niveau des sites de contrôle et d'intervention après le lancement de l'intervention.

Dans certains cas où, le volume de données disponibles est très important avant les recherches sur le terrain, seul un pré-appariement peut s'avérer nécessaire. Dans d'autres situations, il suffira uniquement d'effectuer un processus 
de post-appariement. L'Encadré 1 présente un cas emblématique dans lequel on dispose de peu d'informations au niveau du village avant d'effectuer les recherches sur le terrain.

\section{Types d'appariement}

Le concept d'appariement fait en général référence aux méthodes statistiques d'appariement par score de propension et par covariables. Ces méthodes peuvent être mises en œuvre au cours du processus de pré-appariement ou celui de postappariement, à condition que toutes les exigences concernant les données soient respectées pour l'étape donnée. Pour effectuer le pré-appariement d'échantillons à effectifs réduits, ce processus statistique peut être réalisé par un « appariement manuel ». Indépendamment de la méthode, l'objectif est de sélectionner des échantillons d'unités d'intervention et d'unités de contrôle dont les caractéristiques présentent une distribution semblable (c’est-à-dire équilibrée).

\section{Appariement manuel}

Parmi les différents processus d'appariement, l'appariement manuel est le plus simple (et le moins précis). Dans cette méthode, les unités

\section{Encadré 1. Pré-appariement pour la sélection des échantillons dans le cadre de l'étude GCS-REDD+ du CIFOR}

Des enquêtes auprès des ménages et des villages sont effectuées sur 4 villages d'intervention et sur 4 villages de contrôle au niveau de tous les sites du projet de REDD+ faisant partie de l'étude GCS REDD+ du CIFOR. La sélection de ces villages est fondée sur une estimation préalable de leurs principales caractéristiques et un processus d'appariement statistique. Les deux processus d'estimation et d'appariement tiennent plus particulièrement compte des caractéristiques susceptibles d'affecter à la fois la participation des villages d'intervention au projet de REDD+ et les résultats étudiés (bien-être économique et déboisement). Le processus se déroule comme suit :

1. Déterminer jusqu'à 16 villages candidats pour l'intervention. Pour les projets qui couvrent une zone géographique importante, identifier un ensemble de villages pour lesquels des interventions directes du projet sont prévues et où les taux de déforestation récents atteignent ou dépassent la moyenne de la région du projet.

2. Déterminer jusqu'à 16 villages candidats pour servir de contrôle. D'un point de vue géographique, ils doivent être suffisamment proches pour être soumis à des conditions biophysiques ou de marché relativement semblables, mais également suffisamment éloignés pour ne pas subir en principe de retombées ou de fuites directes émanant du projet.

3. Recueillir les données concernant les 22 caractéristiques essentielles qui sont susceptibles d'influencer à la fois le placement des sites dans le cadre du projet ainsi que les résultats concernant l'utilisation des terres et le bien-être économique ; ceci, en se fondant sur l'analyse de données secondaires, I'opinion des informateurs principaux et des visites dans les villages. Pour le processus d'appariement, les caractéristiques qui s'avèrent avoir le plus d'influence étaient : (1) les pressions de déforestation ; (2) la présence d'ONG ; (3) le droit de propriété dans les forêts ; (4) le nombre d'organisations dans le village ; (5) la population ; (6) le couvert forestier du village ; (7) la dépendance vis-à-vis de la forêt et (8) la distance jusqu'à une route principale.

4. Apparier les villages de traitement et de contrôle en associant les séries de covariables (selon la distance de Mahalanobis) : ce processus s'applique à tous les sites du projet dans un pays donné et à toutes les caractéristiques pour lesquels on a toutes les données et suffisamment de variation entre les projets.

Dans l'étude GCS-REDD+, les chercheurs ont choisi d'effectuer l'appariement au niveau du pays, en identifiant les meilleurs groupes de villages de traitement et de contrôle, plutôt que d'apparier les villages un-à-un au niveau du projet. Cette approche offre l'avantage d'augmenter les effectifs d'échantillonnage pour l'appariement et garantit que même si un village est éliminé de l'étude (à savoir qu'il n'a plus le statut de « contrôle » ou d'« intervention »), la paire correspondante ne disparaît pas de l'ensemble des échantillons. 
sont appariées de manière intuitive, soit en tenant compte de leur caractère d'ensemble (approche holistique) ou en se fondant sur des variables d'appariement choisies en faisant preuve d'un jugement avisé et dont la mesure n'est qu’approximative en général. L'essentiel est que ces variables d'appariement, ou encore le caractère d'ensemble des unités, soient pertinents à la fois pour le placement dans le projet et la participation à l'intervention ainsi que les résultats étudiés. Dans sa forme la plus élémentaire, l'appariement manuel peut se résumer à interroger des personnes au niveau des sites d'intervention sur les villages qui ressemblent le plus au leur. Ce processus peut reposer sur une revue de littérature et des données secondaires disponibles relatives aux variables d'appariement (densité de population, distance jusqu'aux routes, potentiel agro-écologique par ex.). Même si cette approche est semblable aux autres formes d'appariement dans ses principes, elle risque d'être faussée par le biais des chercheurs.

\section{Appariement par score de propension et par covariables}

En principe, l'appariement par covariables peut être considéré comme l'équivalent statistique de l'appariement manuel. Cette méthode consiste à apparier des unités de contrôle et des unités d'intervention en tenant compte de la " distance " qui sépare les variables susceptibles d'influencer le(s) résultat(s) étudié(s), et donc d'en fausser l'interprétation (covariables). Il existe plusieurs types de mesures pour quantifier cette distance et des coefficients permettant de limiter les écarts entre les unités appariées. L'appariement par score de propension (ASP) est probablement la méthode la plus souvent utilisée dans le cadre d'évaluations d'impact. Le score de propension est établi en utilisant un modèle statistique permettant de calculer la probabilité de la participation prévue (intervention de REDD+ par ex.) compte tenu de caractéristiques observables. Chaque unité (qu'il s'agisse d'un site forestier, d'un village ou d'un ménage) se voit attribuer son propre score de propension. Les distributions des deux scores de propension (unité de contrôle et unité d'intervention) sont alors indiquées sur un graphe afin de mettre en évidence leur aire de recouvrement (connue sous le nom de : « appui commun »). L'appariement peut être restreint à cette zone d'appui commun. Par exemple, chaque unité d'intervention peut être appariée à une unité de contrôle présentant le score de propension le plus proche (connu sous le nom de : "plus proche voisin »). Cette opération peut avoir lieu avec ou sans remise, et avec ou sans " restriction » (calliper), laquelle impose une distance maximum autorisée entre les « voisins ». Il existe également beaucoup d'autres méthodes qui établissent statistiquement des unités de contrôle pour chaque unité d'intervention (ou du moins chaque unité d'intervention de la zone d'appui commun). En règle générale, la méthode ASP exige de disposer d'un vaste réservoir d'unités de contrôle potentielles ainsi que des données concernant de nombreux facteurs susceptibles d'affecter à la fois le placement dans l'intervention et la participation à l'intervention ainsi que le(s) résultat(s) étudié(s). L'objectif est donc d'identifier un sous-groupe d'unités de contrôle et d'intervention qui sont similaires compte tenu de tous les facteurs. Il existe plusieurs critères permettant d'apprécier cet équilibre. Examiner les histogrammes et les courbes de densité de scores de propension reste l'approche la plus simple. Cette étape doit être réalisée en premier pour l'ensemble des échantillons (non appariés). Les résultats de cette analyse peuvent mettre en évidence le fait que certaines unités d'intervention ont une très petite aire de recouvrement avec les unités de contrôle; ce qui signifie que certaines unités d'intervention ont des particularités si prononcées quaucune unité de contrôle appropriée leur correspondant n’a été découverte. Il s'agit d'une difficulté concrète substantielle à laquelle il faut répondre. Les caractéristiques des unités qui sont finalement écartées de l'analyse doivent toutefois être prises en considération dans l'interprétation des résultats.

\section{Analyse et restrictions}

L'appariement est une manière d'identifier un échantillon de villages et/ou de ménages. Il s'agit d'établir quelles sont les unités d'intervention et les unités de contrôle à inclure (appui commun) et le poids respectif à attribuer à chaque unité de contrôle. Après l'appariement, de nombreuses méthodes peuvent être employées pour évaluer l'impact. Dans la plupart des programmes 
statistiques, les techniques d'appariement permettent de calculer la différence de la moyenne des résultats attribués au projet ( l'effet moyen du traitement sur les traités » ou ATT par ex.). Des méthodes de régression permettent de corriger cette estimation d'impact en fonction du biais ; il est également possible d'envisager des modèles de régression multiple au moyen de l'échantillon apparié. L'appariement associé à la régression est en général considéré comme une méthode fiable d'évaluation de l'impact causal.

Lorsque les facteurs importants qui déterminent le placement dans un projet et la participation à ce projet de REDD+ ainsi que les résultats étudiés sont tous observables (connus et mesurés par les chercheurs), l'appariement est efficace pour filtrer les effets des variables potentiellement confusionnelles. Cependant, en présence de caractéristiques non observables (celles qui sont inconnues du chercheur ou qu'il ne peut pas facilement mesurer) ayant un effet sur le placement/la participation ainsi que les résultats du projet de REDD+, la validité des résultats obtenus au moyen de l'appariement est alors remise en cause. La motivation ou le dynamisme d'un chef de communauté, entre autres, sont des caractéristiques non observables importantes. $\mathrm{Si}$ des données existent concernant les critères de jugement utilisés pour évaluer les résultats avant et après l'intervention, ce problème est alors en partie minimisé.

\section{Retombées}

Le problème des retombées (à savoir les fuites) mérite qu'on s'y intéresse pour terminer cette discussion. Dans le contexte de la déforestation et de la dégradation forestière, le problème des fuites est clairement élucidé. Le projet de REDD+ doit mettre en évidence le fait que la diminution du déboisement et de la dégradation sur le site du projet n'entraîne pas de fuites (à savoir une déforestation ou une dégradation de la forêt qui seraient déplacées vers des zones extérieures à la zone du projet). Il peut s'agir de fuites affectant le bien-être économique en plus de celles amplifiant la perte de forêt ; ces retombées proviennent du site de REDD+ et se déportent au niveau d'autres zones. Par exemple, le volume d'emplois de la filière du bois disparaissant au niveau du site de REDD+ pourrait se déplacer vers une autre zone (et avec lui la dégradation). Ceci signifie qu'il existe un risque certain que la REDD+ affecte également les sites de contrôle appariés. D'une part, cette situation peut compliquer l'interprétation de toute comparaison entre les sites d'intervention et les sites de contrôle. En retour, la détermination des fuites affectant à la fois le bien-être économique et la perte de la forêt est un élément crucial pour la compréhension des phénomènes, il est donc particulièrement intéressant de pouvoir en extraire des données. Les porteurs de projets auront au préalable déterminé le périmètre de fuites affectant le déboisement et la dégradation de la forêt, à savoir les zones tampons à proximité du site du projet où on anticipe ces fuites. De la même manière, il sera utile de déterminer le périmètre de retombées affectant le bien-être économique. Dans la mesure du possible, les sites de contrôle seront situés dans la zone située à l'extérieur du périmètre de fuites, toutefois des données doivent aussi être recueillies dans le périmètre de fuites afin de pouvoir analyser les retombées et les fuites.

\section{Ressources statistiques}

- Commandes sous logiciel STATA pour l'appariement : pscore.ado ; consulter Khandker et al. (2010) pour plus de détails

- R code pour l'appariement : consulter http:// sekhon.berkeley.edu/matching/ 
Fiche technique 8

\section{Étude des liens de causes à effets entre l'intervention et les résultats}

\section{Introduction}

L'analyse de l'enchaînement des causes et des effets du projet, également dénommée « théorie du changement », permet d'expliciter les rapports de causalité entre les interventions de REDD+ et les résultats qu'elles produisent. Ce processus comporte plusieurs étapes, entre autres : la compréhension du contexte, la clarification des objectifs des interventions de REDD+ et de leur mise en œuvre, l'élaboration d'hypothèses vérifiables, la détermination des données requises et la vérification de ces hypothèses. Les résultats de la vérification des hypothèses doivent servir à rectifier l'inventaire des données requises et les hypothèses initiales concernant les conditions et la mise en œuvre du projet au niveau du site. Ces analyses des relations de causalité sont d'autant plus fiables qu'elles auront été fondées par la littérature scientifique et des données d'origines multiples recueillies à des moments différents. Il est important de souligner que les méthodes participatives et les entretiens avec les informateurs principaux peuvent s'avérer très utiles pour effectuer un suivi de la mise en œuvre du projet lorsque les opérations de recueil de données plus détaillées sont difficiles à réaliser.

\section{Exemple : hypothèse d'intervention de REDD+ dans cinq villages limitrophes d'une aire protégée en Ouganda}

\section{Cette fiche technique présente des} recommandations pour établir une description des rapports de causalité qui relient les interventions de REDD+ aux résultats observés en matière de bien-être économique et social. Pour illustrer ce processus, nous utilisons ici un exemple hypothétique. Cet exemple met en évidence l'éventail des facteurs sociaux, économiques, institutionnels et biophysiques à analyser pour pouvoir élaborer un inventaire exact des rapports de causalité entre l'intervention et les résultats observés en matière de bien-être économique.
Les informations nécessaires à la réalisation de ce travail peuvent être trouvées dans des rapports d'études, dans la littérature grise (rapports de recherches, thèses, brevets, etc.) et auprès des informateurs principaux, ainsi qu'en recueillant des données primaires sur le site du projet de REDD+.

\section{Étape 1 : Connaissance du contexte relatif au site du projet}

Au niveau local, les moteurs du déboisement et de la dégradation de la forêt dans cette zone sont, respectivement, la culture du brûlis par des petits agriculteurs et l'abattage illégal de bois exotiques à forte valeur commerciale par des bûcherons utilisant une scie longue. Dans la zone considérée, il n'y a aucun grand propriétaire ou entreprise qui pourrait influencer le mode d'utilisation des terres. Il y a une forêt communautaire dans chaque village ; les villages sont également attenants à un parc national géré de telle sorte que le tourisme et la biodiversité puissent coexister. Depuis sa création, le parc national subit des empiètements et des dégradations, particulièrement dans les zones tampons situées autour. Le prélèvement de tout produit quel qu'il soit à l'intérieur du périmètre du parc nest toléré qu’avec une autorisation de l'Autorité des espèces sauvages d'Ouganda (Uganda Wildlife Authority); la récolte, la cueillette ou le ramassage à titre commercial sont strictement interdits. Le domaine foncier est régi par le droit coutumier ; les propriétaires terriens possèdent des droits fonciers assez bien reconnus sur leurs terres agricoles. La loi ougandaise ne fait état d'aucun droit de jure concernant les arbres ou le carbone. Les droits informels sont déterminés par le droit coutumier, avec chevauchement fréquent des revendications relatives aux ressources. La surveillance et le contrôle de l'application des lois relatifs à l'utilisation de la forêt sont minimaux en raison du manque de ressources des organismes gouvernementaux et des organismes de conservation qui sont actifs dans cette zone. Les petits propriétaires subsistent principalement 
grâce à l'agriculture, tout en pratiquant l'élevage d'un petit cheptel et la récolte des produits tirés de la forêt destinés à la consommation familiale ou à la vente. Le tourisme apporte des revenus aux villages grâce à un système de partage des avantages organisé au niveau paroissial. En raison des conflits et du manque de débouchés économiques au Rwanda et en République démocratique du Congo, l'afflux de migrants provenant de ces régions est particulièrement important. Le nombre de ménages dont le chef est une femme ou qui ne possèdent pas de terres est relativement élevé dans la communauté. Dans un des villages d'intervention, il y avait un groupe de gestion concerté qui n'existe plus aujourd'hui. La densité de la population est particulièrement élevée ( $>250$ personnes au kilomètre carré) ; l'accès au marché est faible et le potentiel agricole est limité en raison de la dégradation des sols et des fortes pentes du terrain dans la région.

\section{Étape 2 : Clarification des objectifs de I'intervention}

Le porteur du projet de REDD+ est une ONG internationale de conservation qui travaille dans la région depuis plus de dix ans. Cette organisation a recueilli des données relatives à la biodiversité et la déforestation ; elle a également formé plusieurs membres de la communauté aux activités de surveillance et aux opérations assurant le respect des limites du parc. Toutefois, la mesure dans laquelle ces personnes dénoncent les activités illégales reste floue. Les activités de l'intervention de REDD+, subventionnée pendant cinq ans par un donateur bilatéral, s'inscrivent dans trois volets principaux dont l'objectif est de réduire la déforestation au niveau du site du projet :

- Activité A : plantation d'arbres pour démarquer les limites du parc avec, entre autres, un système traditionnel taungya (à savoir les propriétaires de terrains qui vivent à proximité du parc ont le droit de cultiver sur la zone tampon de part et d'autre de la limite du parc en échange de l'entretien des arbres et de la surveillance des agriculteurs qui pratiquent le brûlis et empiètent sur les limites du parc);

- Activité B : création d'emplois de garde forestier à temps partiel pour cinq personnes de chaque village avec pour mission la surveillance des coupes de bois dans les aires protégées et la forêt communautaire ;

- Activité C : mise en place d'un fonds de carbone communautaire ; en contrepartie de la réduction avérée du déboisement dans le site du projet, des subventions seront octroyées annuellement à un fonds de carbone communautaire géré par le porteur du projet. Les chefs de village doivent soumettre des propositions pour obtenir des subventions destinées à des projets axés sur la collectivité (installation d'un moulin à céréales, achat de fournitures/matériels pour l'école locale, implantation d'une pépinière de fruitiers par ex.).

\section{Étape 3 : Énonciation d'hypothèses vérifiables}

Les hypothèses émergent à partir de différentes versions d'une simple interrogation, à savoir : "Quels sont les impacts sociaux du projet de REDD+ ? ». Une hypothèse est une proposition scientifique fondée ou une approximation raisonnée qui s'intéresse à la relation anticipée existant entre deux variables. Les hypothèses sont toujours soumises à deux exigences : elles doivent correspondre à des faits connus et elles doivent être vérifiables (Est-ce-que la variable est directement mesurable ou a-t-on besoin d'une variable de substitution ? Peut-on obtenir les données nécessaires pour vérifier les hypothèses en dépit des contraintes associées au calendrier ou aux ressources? L'amplitude de la variation des données est-elle suffisante pour permettre de vérifier l'hypothèse ?). Lors de l'énonciation des hypothèses, il faut impérativement tenir compte des différences entre la mise en œuvre concrète de l'intervention de REDD+ et les prévisions initiales (à savoir si les activités réalisées sur le terrain reflètent les activités prévues à l'origine) (Tableau 1).

\section{Étape 4 : Inventaire des données nécessaires}

La nécessité d'obtenir certaines données pose des questions cruciales.

1. De quelles données a-t-on spécifiquement besoin pour vérifier les hypothèses (à savoir, quels sont les indicateurs ou les variables requises) ? 


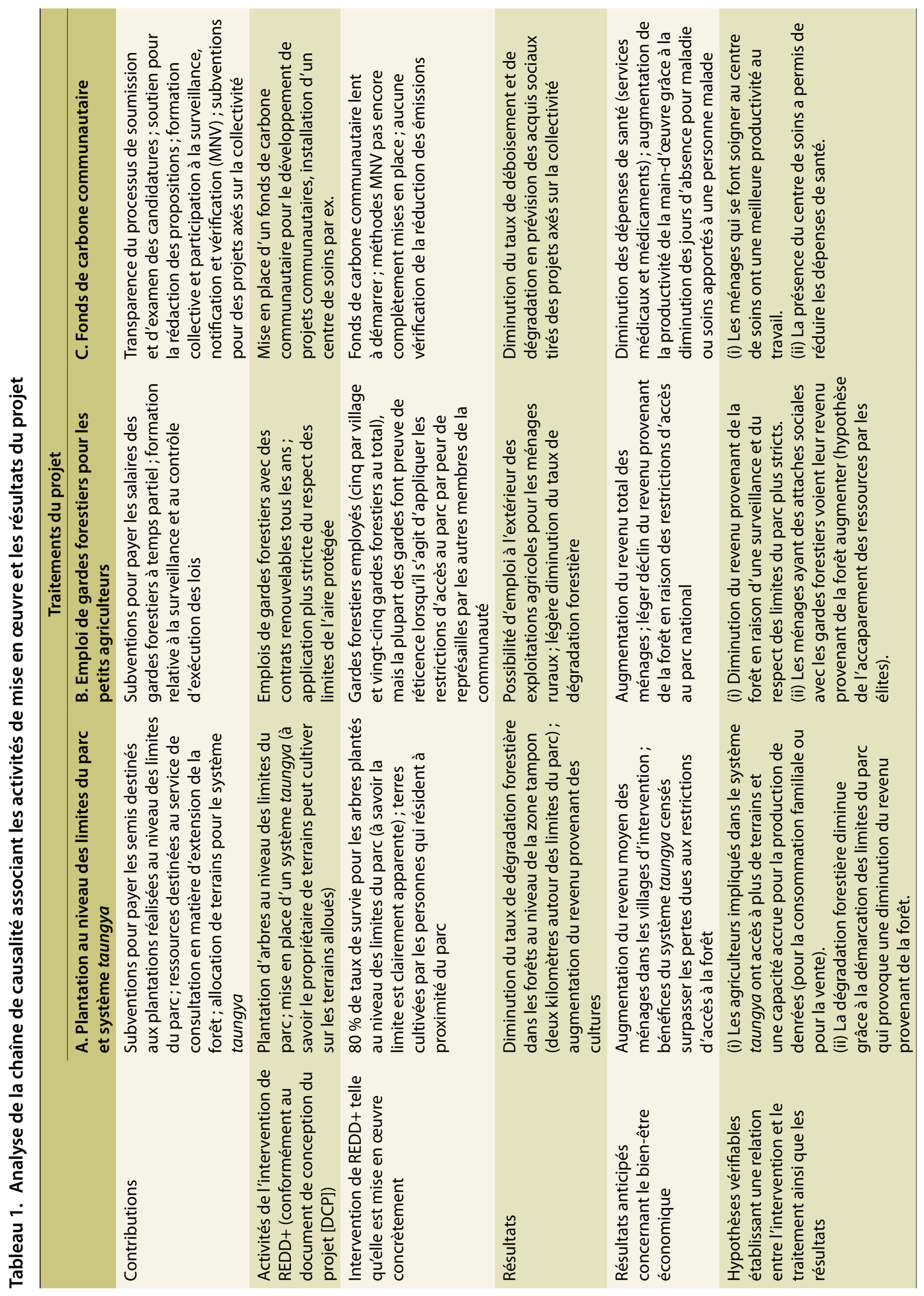


2. Sur quelle échelle prévoit-on d'observer une variation de l'indicateur ou de la variable?

3. Les indicateurs les plus appropriés, sont-ils de nature qualitative ou quantitative? (Tableau 2 ; consulter également la Fiche technique 10.)

En ce qui concerne l'inventaire des données requises, il faut décider si l'on recueille les données à la fois avant et après l'intervention et aussi à la fois sur le site de l'intervention et le site de contrôle.

\section{Étape 5 : Vérification des hypothèses}

Les données qualitatives peuvent servir à structurer une présentation de la relation (ou corrélation) qui existe entre les variables et qui est suggérée dans l'hypothèse. Lorsque les données quantitatives sont recueillies au niveau d'un échantillon suffisamment important (à savoir $\mathrm{N} \geq 80$ ), les méthodes de corrélation ou d'analyse de régression peuvent servir à vérifier les relations qui existent entre les variables. En cas d'obtention de résultats inattendus, on effectuera une analyse plus poussée des contributions, des activités et des résultats de la mise en œuvre du projet ainsi que de leur lien avec les résultats. Il faut garder à l'esprit la première question sur laquelle est fondée l'hypothèse: Quel est l'impact de l'intervention de REDD+sur le bienêtre économique et social ? Pour bien comprendre les phénomènes de causalité qui conditionnent les rapports entre l'intervention et les résultats au fil du développement d'un projet de REDD+, les données sur lesquelles s'appuie l'analyse de la chaîne de causalité devront être mises à jour et rectifiées.

\section{Tableau 2. Besoins en données qualitatives et quantitatives pour vérifier les hypothèses}

\begin{tabular}{|c|c|c|c|c|c|c|}
\hline & \multicolumn{6}{|c|}{ Traitements prévus par le projet } \\
\hline & \multicolumn{2}{|c|}{$\begin{array}{l}\text { A. Plantation d'arbres sur les } \\
\text { limites et taungya }\end{array}$} & \multicolumn{2}{|c|}{$\begin{array}{l}\text { B. Emplois hors agriculture } \\
\text { comme garde forestier }\end{array}$} & \multicolumn{2}{|c|}{$\begin{array}{l}\text { C. Fonds communautaire du } \\
\text { carbone }\end{array}$} \\
\hline & \multicolumn{2}{|c|}{ Hypothèse (i) et (ii) } & \multicolumn{2}{|l|}{ Hypothèse (i) et (ii) } & \multicolumn{2}{|c|}{ Hypothèse (i) et (ii) } \\
\hline & $\begin{array}{l}\text { Données } \\
\text { qualitatives }\end{array}$ & $\begin{array}{l}\text { Données } \\
\text { quantitatives }\end{array}$ & $\begin{array}{l}\text { Données } \\
\text { qualitatives }\end{array}$ & $\begin{array}{l}\text { Données } \\
\text { quantitatives }\end{array}$ & $\begin{array}{l}\text { Données } \\
\text { qualitatives }\end{array}$ & $\begin{array}{l}\text { Données } \\
\text { quantitatives }\end{array}$ \\
\hline $\begin{array}{l}\text { Données } \\
\text { au niveau } \\
\text { des } \\
\text { ménages }\end{array}$ & $\begin{array}{l}\text { Perception } \\
\text { des bénéfices } \\
\text { et du coût } \\
\text { du système } \\
\text { taungya }\end{array}$ & $\begin{array}{l}\text { Participation } \\
\text { au système } \\
\text { taungya ; } \\
\text { portefeuille } \\
\text { de revenus } \\
\text { (surtout } \\
\text { parts hors } \\
\text { agriculture et } \\
\text { exploitation } \\
\text { forestière) ; } \\
\text { temps } \\
\text { consacré à la } \\
\text { surveillance } \\
\text { par le ménage }\end{array}$ & $\begin{array}{l}\text { Perception de } \\
\text { l'efficacité du } \\
\text { travail des gardes } \\
\text { forestiers }\end{array}$ & $\begin{array}{l}\text { Données sur } \\
\text { le portefeuille } \\
\text { de revenus } \\
\text { (surtout } \\
\text { parts hors } \\
\text { agriculture et } \\
\text { exploitation } \\
\text { forestière) ; } \\
\text { interactions } \\
\text { avec les } \\
\text { gardes } \\
\text { forestiers }\end{array}$ & $\begin{array}{l}\text { Perception de } \\
\text { la santé et du } \\
\text { bien-être par } \\
\text { les membres } \\
\text { du ménage }\end{array}$ & $\begin{array}{l}\text { Nombre de } \\
\text { journées de } \\
\text { maladie; } \\
\text { dépenses } \\
\text { de santé ; } \\
\text { productivité } \\
\text { au travail du } \\
\text { ménage ; accès } \\
\text { au centre de } \\
\text { soins }\end{array}$ \\
\hline $\begin{array}{l}\text { Données } \\
\text { au niveau } \\
\text { des } \\
\text { villages }\end{array}$ & $\begin{array}{l}\text { Perception } \\
\text { du système } \\
\text { taungya }\end{array}$ & $\begin{array}{l}\text { Nombre } \\
\text { d'infractions } \\
\text { dans la zone } \\
\text { tampon }\end{array}$ & $\begin{array}{l}\text { Expérience } \\
\text { actuelle et } \\
\text { passée en } \\
\text { matière de } \\
\text { mainmise par les } \\
\text { élites }\end{array}$ & $\begin{array}{l}\text { Nombre } \\
\text { d'infractions } \\
\text { enregistrées } \\
\text { par les gardes } \\
\text { forestiers }\end{array}$ & $\begin{array}{l}\text { Préférence } \\
\text { pour des } \\
\text { services de } \\
\text { santé ou pour } \\
\text { la médecine } \\
\text { tradition nelle }\end{array}$ & $\begin{array}{l}\text { Nombre de } \\
\text { visites au } \\
\text { centre de soins }\end{array}$ \\
\hline
\end{tabular}




\section{Fiche technique 9}

\section{Analyse de la répartition}

\section{Introduction}

Comprendre la mesure dans laquelle les interventions de REDD+ produisent des impacts qui peuvent être différents selon les groupes est une étape essentielle avant d'appréhender les perspectives d'équité et de co-bénéfices qu'offre la REDD+ainsi que de connaître les gagnants et les perdants. Par exemple, les impacts du projet sont-ils ressentis différemment en fonction de l'appartenance à différents groupes socioéconomiques, ethniques ou encore par les hommes ou par les femmes? L'analyse de la répartition dispose de plusieurs méthodes et instruments permettant d'évaluer la manière dont les impacts varient d'un groupe à l'autre; cette méthode apporte également des réponses aux questions précédentes.

Si les personnes qui vivent en zone rurale ne peuvent plus accéder à la forêt et en retirer certains produits, ceci peut avoir un impact considérable dans le cas où la proportion relative de leur revenu provenant de la forêt ou de la consommation de produits de la forêt était élevée avant l'intervention. Des études menées au cours des dix ou quinze dernières années établissent clairement que les ménages pauvres et vulnérables sont particulièrement dépendants vis-à-vis des produits de subsistance provenant de la forêt, alors qu'au contraire les ménages relativement fortunés disposent d'un capital financier et social leur permettant de tirer profit des marchés de produits forestiers à forte valeur commerciale (Cavendish 2000, Arnold 2002, Bush et al. 2004, Fisher 2004, Vedeld et al. 2004, Narain et al. 2005, Chomitz et al. 2007). De plus, les plus démunis dépendent des forêts car ces dernières jouent le rôle d'un filet de sécurité pendant les périodes de crise économique ou sociale ; les forêts permettent également de satisfaire aux besoins de consommation courants de ces ménages ruraux (Pattanyak et Sills 2001, Angelsen et Wunder 2003). D'ailleurs, nous savons également que le revenu provenant de la forêt et de l'environnement a un effet compensateur sur les inégalités dans les sociétés rurales. D’après les estimations classiques des inégalités de revenus faites à partir de données caractérisant les portefeuilles de revenus, avec ou sans apport provenant de la forêt et de l'environnement, il est clair que l'accès aux produits tirés de la forêt compense les inégalités entre les ménages, inégalité qui serait manifeste en l'absence de revenu provenant de la forêt (Lopez-Feldman et al. 2007, Cavendish et Campbell 2008, Jagger sous presse). Les preuves émanant de ces études empiriques justifient l'analyse de la variabilité de l'impact des interventions de REDD+ (Encadré 1).

L'analyse de la répartition peut s'effectuer de différentes façons : elle s'appuie notamment sur les résultats qualitatifs concernant les effets des interventions sur certains groupes, des méthodes de statistique descriptive qui permettent d'analyser les impacts par groupe, des techniques de mesure de la pauvreté ou des inégalités entre sous-groupes ; elle intègre ensuite des variables d'interaction qui représentent les sous-populations concernées dans un calcul de régression à plusieurs variables (pour analyser les conséquences d'être, à la fois, une femme et pauvre, ou la manière dont les facteurs de pauvreté et d'appartenance sexuelle interagissent et affectent l'impact de l'intervention par ex.).

\section{Groupes de consultation}

L'organisation de groupes de consultation et les entretiens avec des informateurs principaux sont indispensables pour déterminer les groupes susceptibles d'être affectés de manière différente par les interventions de REDD+ sur le plan du bien-être économique. Il est possible de recueillir des informations de nature qualitative concernant les groupes marginalisés d'un point de vue social ou économique ; cette collecte de données a lieu à différents moments au cours du suivi et du processus d'évaluation. Les discussions des groupes de consultation avant le début des interventions 


\section{Encadré 1. L'étude GCS-REDD+ et la répartition des impacts}

De solides preuves confirment que l'accès à des produits diversifiés provenant de la forêt est un facteur très important pour l'économie de subsistance des groupes ruraux pauvres, des femmes et autres groupes vulnérables. Si la mise en œuvre d'un projet de REDD+ conduit à une augmentation de la distance que les femmes ont à parcourir pour aller chercher du bois de chauffage, ou encore cueillir les plantes médicinales, récolter les aliments naturels, matériaux d'artisanat et autres produits de la forêt, c'est la subsistance même de ces groupes qui est remise en cause

L'étude GCS-REDD+ utilise différentes méthodes pour expliciter l'effet des interventions de REDD+ sur les populations pauvres et vulnérables. Par exemple, au niveau des villages, des groupes de consultation, incluant des femmes représentatives des caractéristiques démographiques de tout le village, permettent de mieux comprendre non seulement la manière dont les femmes utilisent et entretiennent les forêts, mais aussi le rôle des femmes dans la mise en œuvre des projets de REDD+, la manière dont le projet les affecte et la façon dont elles prennent connaissance de la REDD+ (consulter le Questionnaire destiné aux femmes - Annexe C).

Au niveau du ménage, l'étude GCS-REDD+ recueille des données en vue de former un échantillon représentatif des ménages dans chaque village (Questionnaire destiné aux ménages - Annexe C). L'évaluation des résultats concernant le bien-être économique de certains sous-groupes, comme les migrants par opposition aux habitants installés depuis longtemps, nous permet de vérifier les hypothèses selon lesquelles les interventions de REDD+ ont un impact différent sur les nouveaux arrivants. Des techniques statistiques plus élaborées et reposant sur des variables d'interaction, par exemple, en combinant la variable de traitement avec une variable représentative des ménages migrants de fraîche date nous permettent d'évaluer l'effet de la réforme sur les ménages migrants tout en tenant compte de l'effet des covariables.

de REDD+ donnent accès à d'importantes informations sur les groupes qui dépendent de la forêt et d'autres groupes vulnérables ; cette activité garantit de recueillir des données pertinentes « avant »et « après », lesquelles permettent d'analyser la variabilité des impacts sur le bien-être économique. Les groupes de consultation peuvent également être utilisés pour recueillir des données rétrospectives ; les participants présentent d'une manière qualitative leurs opinions sur la façon dont les groupes vulnérables sont affectés depuis le début de la mise en œuvre du projet. Les groupes de consultation sont indispensables pour comprendre le processus par lequel les interventions affectent les groupes pauvres et vulnérables. Les discussions de groupe produisent des données qui révèlent certains des mécanismes à l'origine de la variabilité des impacts observés dans les évaluations quantitatives mesurant l'évolution du bien-être économique.

\section{Méthodes mixtes et répartition des impacts}

En règle générale, la documentation au sujet des premiers projets de carbone forestier ainsi que celle relative aux impacts sur le bien-être économique des projets de conservation et de développement, est, par essence, de nature qualitative (Caplow et al. sous presse). Les données générées par les discussions au sein des groupes de consultation et les entretiens avec des informateurs principaux sont indispensables pour, d'une part, produire des informations utiles permettant de définir qui sont les groupes vulnérables mais également dresser un premier tableau sur la manière dont ces derniers sont affectés par l'intervention. L'analyse quantitative est un instrument puissant complétant le contenu des récits dans lesquels des ménages échappent à la pauvreté, les ressources sont accaparées par les élites et les groupes vulnérables sont enfermés dans l'engrenage de la pauvreté. À l'aide d'une technique élémentaire de statistique descriptive, de calculs de régression à plusieurs variables pour évaluer l'hétérogénéité de l'impact et d'estimations de l'effet de l'intervention sur les inégalités au sein de la communauté, nous sommes capables de présenter, en toute confiance et avec une précision accrue, l'amplitude de la répartition des impacts. 


\section{Analyse des résultats concernant le bien-être économique}

Pour présenter la manière variable dont les groupes vulnérables sont affectés par les interventions de REDD+, on dispose de plusieurs moyens permettant de décomposer, au niveau du ménage, les données des résultats relatifs au bien-être économique, incluant, par exemple, le quartile de revenu, l'appartenance à un groupe ethnique, la dimension hommes-femmes ou le statut de migrant. En produisant des tableaux où figurent, regroupées en catégories, les moyennes des résultats relatifs au bien-être économique, on peut facilement vérifier les hypothèses émises sur les différences significatives de résultats entre les différents sous-groupes de l'échantillon. Par exemple, la décomposition des données relatives au bien-être selon le sexe du chef de ménage s'avère être une méthode efficace pour identifier la manière dont les interventions affectent différemment les hommes et les femmes.

\section{Analyse de I'hétérogénéité de l'impact par des méthodes de régression à plusieurs variables}

La statistique descriptive et les tests de différences de moyennes produisent des résultats descriptifs. En revanche, le calcul de régression à plusieurs variables tient compte des covariables et des variables de confusion susceptibles d'influencer les résultats; il permet donc de produire une image plus concrète de l'effet des interventions de REDD+ sur les groupes pauvres et vulnérables. Il existe deux façons d'aborder la technique de régression à plusieurs variables. Dans la première, on scinde l'échantillon de ménages représentatifs en fonction de la variable qui représente le groupe vulnérable (variable dépendante). Les calculs de régression réalisés pour chaque sous-groupe produisent une estimation de l'effet de l'intervention sur les résultats relatifs au bien-être économique du groupe vulnérable (consulter Jagger 2008 qui donne un exemple de cette méthode appliquée à l'analyse des impacts sur le bien-être économique de la réforme du secteur forestier en Ouganda). Il existe une autre méthode permettant d'évaluer l'effet d'une intervention de REDD+, elle passe par la création d'un terme d'interaction (à savoir une nouvelle variable) qui permet de combiner la participation à l'intervention (à savoir dans le groupe d'intervention $[\mathrm{CI}])$, dans le groupe " après » [BA] ou dans les deux groupes après/intervention $[\mathrm{BACI}])$, à la variable dépendante qui représente la population vulnérable (Plewis 2002, Khandker et al. 2010). Par exemple, à l'aide de la méthodologie d'analyse BACI ou CI, pour expliciter la manière dont une intervention de REDD+ a affecté les ménages dont le chef est une femme, il suffit de créer une nouvelle variable fictive REDDFhead

\section{Encadré 2. Calcul de Gini pour mesurer les inégalités de revenus}

Le coefficient de Gini est une mesure de I'inégalité de la distribution des revenus. Pour mieux comprendre le rôle déterminant du revenu provenant de l'environnement, le coefficient de Gini peut être décomposé en fonction de l'origine du revenu en vue de calculer l'impact que peut avoir une variation mineure du revenu provenant de la forêt et de l'environnement sur les inégalités (pour trouver des exemples, consulter Jagger sous presse et Lopez-Feldman et al. 2007). Selon Lerman et Yitzhaki (1985), le coefficient de Gini correspondant au degré d'inégalité du revenu total, G, peut être représenté de la manière suivante :

$$
G=\sum_{K=1}^{K} S_{k} G_{k} R_{k}
$$

Où : $S_{k}$ représente la proportion du composant $k$ dans le revenu total, $G_{k}$ est le coefficient de Gini de la source, il correspond à la répartition du revenu provenant de la source $k$, et $R_{k}$ est le coefficient Gini de corrélation entre le revenu provenant de la source $k$ et la répartition du revenu total. Le calcul de Gini nous renseigne sur le poids d'une source de revenu dans le revenu total $\left(S_{k}\right)$, si la source de revenu est répartie de façon égale ou inégale $\left(G_{k}\right)$ et la manière dont la source de revenu et la répartition du revenu total entrent en corrélation $\left(R_{k}\right)$. Le dernier terme, $R_{k^{\prime}}$ rend compte de l'importance avec laquelle le revenu en provenance de l'environnement favorise ou défavorise les populations pauvres. Pour trouver plus d'informations sur le calcul de Gini et l'obtention d'effets marginaux à l'aide de la commande « descongini » sur le logiciel STATA, consulter Lopez-Feldman (2006). 
qui prend la valeur « 0 » si le ménage se trouve à l'extérieur du groupe de traitement et si le chef du ménage est un homme, et la valeur « $1 »$ si le ménage se trouve sur le site du projet de REDD+ et si le chef du ménage est une femme. Cette méthode permet d'évaluer de manière précise l'hétérogénéité de l'impact du projet en fonction des groupes vulnérables dans un échantillon donné.

\section{Mesure des inégalités comme indication de la nature des impacts concernant le bien-être économique sur des échantillons entiers ou des sous-groupes}

Parmi toutes les méthodes d'analyse du revenu ou du niveau d'inégalité des richesses, le coefficient de Gini, une mesure de la distribution statistique, est le plus fréquemment utilisé pour mesurer les inégalités de revenus (Encadré 2). Le coefficient de Gini est un nombre variant de « 0 » à « 1 » où les coefficients les plus élevés reflètent un niveau plus élevé d'inégalité de revenus. Le rôle important joué par le revenu provenant de la forêt ou de l'environnement dans l'égalisation sociale permet de supposer qu'une comparaison des estimations des coefficients de Gini au niveau des sites d'intervention et de contrôle, à l'aide des données « avant » et « après » ou des données BACI, peut montrer si les interventions de REDD+ ont un effet compensateur en ce qui concerne les inégalités des revenus qui affectent les ménages en zone rurale. 


\section{Fiche technique 10}

\section{Définition des variables}

\section{Introduction}

Les modèles de causalité et les théories du changement jouent un rôle fondamental dans la détermination des variables qu'il faut prendre en considération dans le cadre de l'analyse. Les modèles font en général référence à quatre types de variables importantes dont l'étude permet de tirer les leçons de l'expérience des projets REDD+ de première génération. Il s'agit des (1) critères de jugement, (2) des variables explicatives, (3) des variables de confusion et (4) des variables de processus. Le modèle de causalité permet non seulement de déterminer les variables pertinentes pour l'analyse mais aussi d'anticiper leurs relations et leurs boucles de rétroactions. Avant de commencer à recueillir des données destinées à caractériser les variables nécessaires à la vérification des hypothèses émanant des modèles de causalité, il faut réfléchir attentivement aux questions soulevées par le concept d'échelle. La plupart des analyses d'interventions de REDD+ nécessitent des variables qui correspondent à des phénomènes se déroulant

\section{Encadré 1. Choisir une méthode d'enquête}

Une fois précisé le type de données nécessaires, il se pose la question du choix de la méthode la plus adaptée au recueil de ces données. Nous indiquons ici deux questions qui nous semblent cruciales pour déterminer la manière la plus efficace d'envisager le recueil des données.

1. Cette variable est-elle susceptible de fluctuer à l'échelle du village ou de la communauté ? Si « oui », les informations doivent être relevées à l'échelle du ménage. Dans le cas contraire, elles peuvent être recueillies à l'échelle du village.

2. Est-il possible de relever des mesures quantitatives fiables en rapport avec cette variable, ou mieux, a-t-on besoin de mesures quantitatives correspondant à cette variable ? Si la réponse à ces deux questions est « oui », les questions concernant cette variable doivent figurer dans l'enquête sur les ménages. Dans le cas contraire, choisissez les informateurs principaux ou les groupes de consultation/réunions de village.

Les réponses à ces questions permettent de classer l'information recueillie dans une des quatre catégories disponibles (Tableau 1).

\section{Tableau 1. Tableau décisionnel concernant la méthode à adopter}

\begin{tabular}{|c|c|c|c|}
\hline & & \multicolumn{2}{|c|}{$\begin{array}{c}\text { Cette variable est-elle susceptible de fluctuer à l'échelle } \\
\text { du village? }\end{array}$} \\
\hline & & Oui & Non \\
\hline \multirow{2}{*}{$\begin{array}{l}\text { Est-il possible d'obtenir des } \\
\text { mesures quantitatives? Ces } \\
\text { mesures sont-elles nécessaires? }\end{array}$} & Oui & $\begin{array}{l}\text { Enquête structurée à } \\
\text { l'échelle du ménage }\end{array}$ & $\begin{array}{l}\text { Enquête structurée à } \\
\text { l'échelle du village }\end{array}$ \\
\hline & Non & $\begin{array}{l}\text { Informateurs principaux, } \\
\text { groupes de consultation }\end{array}$ & Réunions de village \\
\hline
\end{tabular}

Il est indispensable de suivre ces étapes et de sélectionner l'échelle à laquelle les données sont recueillies, puis de vérifier la nécessité d'obtenir des données quantitatives de manière à pouvoir disposer d'informations les plus exactes et précises possibles ; par ailleurs, ce processus est également essentiel afin d'éviter d'importuner les personnes interrogées qui participent à votre enquête. 
à plusieurs niveaux : région infranationale, village, ménage et éventuellement niveau intra-ménage. Lorsque les variables sont construites à partir des données recueillies sur différentes échelles, elles sont dénommées variables multidimensionnelles. Les concepts d'échelle et de variation sont étroitement liés. La question principale soulevée ici se résume à : sur quelle échelle prévoit-on d'observer une variation des données? Prenons comme exemple la consommation électrique ; si les données sont recueillies au niveau d'une zone dans laquelle certains villages n’ont pas accès au réseau électrique alors que d'autres disposent d'électricité, la variation observée au niveau des données aura lieu à l'échelle du village, ce qui signifie que la collecte de données à l'échelle du ménage ne produira aucune variation pour un village donné (Encadré 1).

\section{Critères de jugement}

Lexpérience et les connaissances acquises au cours des projets de REDD+ s'appuient fondamentalement sur le recueil de données relatives aux résultats étudiés. Les critères de jugement doivent être formellement caractérisés afin de pouvoir rendre compte de la manière la plus exacte et précise possible des différentes dimensions des résultats qui nous intéressent ; les données correspondantes doivent donc être recueillies à une échelle qui permet de traduire cet objectif. Si les interventions de REDD+ sont axées sur les ménages, les variables les plus appropriées doivent alors correspondre aux éléments suivants considérés ici à l'échelle du ménage : mesures de revenu, consommation, dépenses, emploi du temps, état de santé et les autres indicateurs habituels qui indiquent le bien-être économique et social (consulter la Fiche technique 2). Ces variables recouvrent les différentes dimensions du bien-être qui sont les plus importantes en fonction de la catégorie à laquelle on appartient au sein du ménage («femme »/« homme » ou « adulte »/« enfant » par ex.). Si les interventions ont lieu à l'échelle du village, l'information qui convient le mieux doit représenter le bien-être à l'échelle du village. Toutefois, comme indiqué ci-dessus, la représentation peut provenir d'une combinaison entre des indicateurs à l'échelle du village et d'autres indicateurs pondérés ou agrégés liés au ménage. Les critères de jugement les plus fiables proviennent de mesures directes comme par exemple, afin d'évaluer le revenu net provenant des produits de la forêt, on mesure la quantité de produits forestiers récoltés par un ménage et on combine cette valeur aux données relatives au prix local et aux coûts supportés par le ménage. Si les coûts de réalisation de mesures directes sont trop élevés où si le temps d'exécution est trop important, on peut utiliser des mesures de substitution, comme une variable de l'échelle de Likert qui permet de rendre compte de la disponibilité d'un produit spécifique, comme le bois de chauffage, dans la zone du projet. Les opinions des usagers locaux de la forêt, des fonctionnaires forestiers et des autres acteurs importants peuvent aussi aider à constituer des variables qui traduisent les résultats de l'intervention de REDD+.

Tableau 2. Calcul de l'effet moyen relatif au bien-être économique et social du projet de REDD+ en se basant sur le revenu

\begin{tabular}{|c|c|c|}
\hline Méthodologie de l'étude & Description & $\begin{array}{l}\text { Formule }(Y=\text { revenu } \\
\mathrm{t}=\text { traitement ou intervention } ; \\
\mathrm{c}=\text { témoin apparié } ; 1=\text { près } ; \\
0=\text { avant })\end{array}$ \\
\hline Randomisation & Contrôle/Témoins du projet & $\left(Y_{t 1}-Y_{c 1}\right)$ \\
\hline Avant-Après/Contrôle-Intervention (BACI) & Différence de différences & $\left(Y_{t 1}-Y_{t 0}\right)-\left(Y_{c 1}-Y_{c 0}\right)$ \\
\hline Avant-Après & Après-Avant & $\left(Y_{t 1}-Y_{t 0}\right)$ \\
\hline Contrôle-Intervention & Intervention-Contrôle & $\left(Y_{t 1}-Y_{c 1}\right)$ \\
\hline Rétrospective & Après ajusté & $\left(Y_{t 1}\right) \times($ variation estimée) \\
\hline
\end{tabular}


À l'aide des données concernant les résultats, il est possible d'évaluer la variation moyenne du bien-être économique et social en calculant les différences entre les groupes, en fonction de la méthodologie expérimentale, des caractéristiques de l'échantillonnage et/ou de celles de l'appariement. Le Tableau 2 présente la manière dont la méthodologie détermine la nature du calcul.

\section{Variables explicatives}

L'étude élémentaire des critères de jugement permet d'avoir un aperçu essentiel, mais incomplet, de la relation entre les projets de REDD+ et les variations observées du bien-être économique et social. Il existe plusieurs autres variables explicatives ou indépendantes qui sont susceptibles d'influencer la variation des critères de jugement. En réalisant un recueil de données concernant les facteurs exogènes (qui ne sont pas influencés par le projet) à plusieurs échelles différentes, il est possible d'étudier leur impact sur les résultats. À l'échelle du ménage, le jeu habituel de variables généralement reconnues comme étant des facteurs déterminants pour le bien-être du ménage comprend : la dotation de terrains, le capital humain (éducation, taille du ménage, taux de dépendance par ex.), le capital financier, le capital social, les actifs (comprenant le cheptel) et le type de domicile. Comme toutes les autres catégories de variables, il est indispensable de tenir compte de l'échelle à laquelle doit avoir lieu le recueil de données correspondant aux variables explicatives. Des variables de plus grande échelle comme l'accès au marché, la densité de population, la vocation agricole et la présence d'infrastructures de soins et d'éducation sont également des bons indicateurs des variations à l'échelle du village susceptibles de conduire à des résultats différents. À l'aide de critères de jugement bien identifiés et des variables explicatives qui leur correspondent, ainsi qu'une variable de traitement pour chacune des unités d'observation, le calcul de régression à plusieurs variables peut servir à évaluer l'effet moyen du traitement (ATE).

\section{Variables de confusion}

Un modèle comportemental simple comprend un critère de jugement (à savoir une variable dépendante) expliqué par un jeu de variables (à savoir des variables explicatives ou indépendantes), dont les variables de projet ou la variable de traitement étudiée. Les critères de jugement nous permettent d'exprimer une opinion (ou un jugement) sur la variation observée et découlant d'un projet de REDD+; de plus, les variables explicatives nous permettent de surveiller les facteurs, autres que ceux associés à l'intervention elle-même, qui sont susceptibles d'influencer le résultat. Certaines de ces variables explicatives sont également des "variables de confusion », car elles permettent d'expliquer non seulement les résultats mais aussi le traitement lui-même. Dans le cadre d'une évaluation d'impact, les variables de confusion présentent un problème significatif: ce sont des facteurs qui, d'une part, expliquent directement le résultat et qui, de plus, sont corrélés, mais non causés par le traitement du projet de REDD+. Il peut s'agir de facteurs déterminant le traitement ou d'éléments non observables qui créent une corrélation entre ces variables explicatives et le traitement. Les variables de confusion posent problème pour tirer des leçons des projets de REDD + car, si elles suggèrent l'existence d'un lien entre le projet de REDD+ et les résultats observés, elles ne nous apprennent rien sur leurs relations de causes à effets. Il est crucial d'engager une réflexion et de rechercher des explications contradictoires aux résultats observés afin de pouvoir déterminer la nature des variables de confusion.

Il existe plusieurs manières d'aborder le problème des variables de confusion, entre autres, on peut inclure des groupes de contrôle soigneusement sélectionnés; ou encore intégrer les variables de confusion observées dans un calcul de régression à plusieurs variables; dans le cas des variables non observées utiliser une variable instrumentale (à savoir une variable qui nentre pas dans l'équation de résolution et qui est corrélée aux variables explicatives endogènes, selon la nature des autres covariables); ou en utilisant une méthodologie de randomisation.

\section{Variables de processus}

Cette présentation des critères de jugement, des variables explicatives et de confusion met l'accent sur la réalisation d'un recueil de données permettant non seulement d'expliquer les résultats observés, mais aussi d'éliminer toute autre 
explication. Ces variables sont indispensables pour mieux connaître les impacts des projets de REDD+ex post. Cependant, ces trois jeux de variables ne nous permettent pas encore de dresser un tableau complet des raisons pour lesquelles certains résultats particuliers sont observés. Dans les études concernant les projets de REDD+, il est indispensable d'élucider ces causes afin de mieux connaître notre sujet. L'identification et la compréhension des maillons de la chaîne de causalité reposent sur l'analyse d'éléments dénommés "variables de processus ». Ces variables décrivent les conditions qui influencent la mise en œuvre du projet (consulter la Fiche technique 8). Les variables de processus sont généralement identifiées et mesurées ex ante dans le cadre du développement de "la théorie du changement " qui relie l'intervention de REDD+ aux résultats qu'elle produit. 


\section{Annexe B. Bibliographie analytique}

Il existe un très grand nombre de guides et d'instruments d'analyse de l'évolution des conditions de vie et de l'utilisation des sols, dont la synthèse dépasse notre propos. L'objectif de cette annexe est plutôt de compléter les guides existants et d'aiguiller le lecteur vers un ensemble diversifié de sources qui contribueront toutes à enrichir le domaine pointu dont traite ce guide, à savoir l'analyse des impacts de la REDD+ sur les populations locales. Les thèmes abordés sont variés et comprennent la détermination des forces complexes à l'origine de la déforestation et de la dégradation des forêts, la définition de scénarios contrefactuels et la détermination des causes des résultats sociaux et environnementaux (attribution), la mesure des changements affectant le bien-être économique, physique et psychologique ainsi que la surface et l'état des forêts, et l'étude des idées que se font les populations des changements d'utilisation des sols. Dans cette optique, cette annexe fournit au lecteur des références essentielles sur (1) les méthodologies de recherche, (2) l'analyse des résultats du point de vue du bien-être économique, physique et psychologique, (3) l'analyse des utilisations des sols et des idées que se font les populations sur l'évolution de ces utilisations, (4) les moteurs de la déforestation et de la dégradation des forêts et (5) la définition de niveaux d'émission de référence, ou niveaux de référence, et des questions connexes liées à la mesure du carbone forestier dans le cadre de la REDD+.

\section{Méthodologies de recherche}

\section{A. Guides d'évaluation d'impact}

Shoestring evaluation: designing impact evaluations under budget, time and data constraints (Bamberger et al. 2004)

Cet article expose une démarche de simplification des méthodologies d'évaluation lorsque des ressources et des données limitées sont un obstacle à l'application des approches optimales. Il présente une synthèse de sept méthodologies d'évaluation dont certaines sont bien développées et d'autres moins solides, guide la réflexion préalable à la prise de décisions relatives à la diminution des effectifs d'échantillonnage, des efforts de collecte de données et des coûts d'analyse, et propose une méthode de reconstitution des données relatives aux niveaux de référence et aux groupes de contrôle. Les auteurs étudient les modalités d'intégration de méthodes participatives, d'application des approches méthodologiques mixtes et de collecte de données dans les cas délicats : thèmes sensibles ou groupes difficiles à atteindre. Ils donnent également des conseils pour le recensement des éléments susceptibles de remettre en cause la validité des méthodologies d'évaluation et des recommandations pour contourner ces difficultés.

Bamberger, M., Rugh, J., Church, M. et Fort, L. 2004 Shoestring evaluation: designing impact evaluations under budget, time, and data constraints. American Journal of Evaluation, 25(1): 5-37.

Evaluation manual: methodology and processes (FIDA 2009)

Bien que ce manuel ait été créé à l'intention de l'équipe d'évaluation du Fonds international de développement agricole (FIDA, organisme spécialisé de l'ONU), certaines des recommandations et des perspectives qui y figurent pourraient intéresser un public plus large. $\mathrm{Ce}$ manuel fournit en effet une liste de techniques de collecte de données considérées comme faisant partie des pratiques d'excellence, mais laisse l'évaluateur libre de son choix. Il remet en cause l'idée de considérer systématiquement les méthodes d'évaluation d'impact expérimentales et quasiexpérimentales comme « la » référence, étant donné leurs difficultés et leurs coûts, surtout dans des situations complexes. Il souligne en revanche l'utilité des approches qualitatives et participatives. L'approche de la FIDA en ce qui concerne l'évaluation des impacts est présentée comme la « combinaison d'analyses contrefactuelles (par le biais de groupes de contrôle par ex.), de techniques 
« avant-après » et de méthodes de triangulation. Elle repose sur la notation de critères différents sur une échelle à six points, les notes étant attribuées en fonction des réponses de l'évaluateur à certaines questions (dont on suppose quelles s'appuient sur une analyse de données obtenues par des enquêtes auprès des ménages, des méthodes participatives ou l'opinion personnelle de l'évaluateur formée par l'examen d'autres sources).

Fonds International de Développement Agricole (FIDA) 2009 Evaluation manual: methodology and processes. Bureau de l'évaluation du FIDA, Rome, Italie. http://www.ifad.org/evaluation/ process_methodology/index htm.

\section{Handbook on impact evaluation: quantitative methods and practices (Khandker et al. 2010)} Ce livre propose une synthèse de fond sur les méthodes d'évaluation expérimentales et quasiexpérimentales et comprend des exercices pratiques (pour une utilisation avec le logiciel de statistique STATA). Il traite également de l'utilisation de modèles économiques dans le cadre de l'évaluation des impacts de mesures politiques de grande envergure, mais aussi de méthodes de mesure de la répartition des impacts (lorsque ceux-ci sont hétérogènes pour les différents sous-groupes de la population affectée). La question des différences entre les évaluations ex ante et ex post est également abordée, ainsi que la complémentarité potentielle de ces deux approches.

Khandker, S.R., Koolwal, G.B. et Samad, H.A. 2010 Handbook on impact evaluation: quantitative methods and practices. Banque mondiale, Washington, DC.

\section{Impact evaluations and development: NONIE guidance on impact evaluation (Leeuw et Vaessen 2009)}

Ce livre est une publication de NONIE, le Réseau des réseaux pour l'évaluation d'impact qui regroupe plusieurs groupes spécialistes de l'évaluation, $\mathrm{y}$ compris ceux de l'OCDE et de l'ONU. Il fournit des recommandations détaillées sur les méthodologies de recherche et la gestion de l'évaluation d'impact, y compris des conseils pour déterminer si une évaluation d'impact est envisageable du point de vue de sa faisabilité et de son coût. Les approches expérimentales, quasi-expérimentales et non quantitatives sont abordées. Les auteurs mettent l'accent sur les synergies entre différentes méthodes quantitatives et qualitatives. Ils préconisent le recours à des approches méthodologiques mixtes, qui permettent de trianguler les informations et par là-même de favoriser une compréhension en profondeur et nuancée des impacts et des phénomènes qui en sont à l'origine. Ce livre fournit également des recommandations sur l'intégration de techniques participatives d'évaluation dans l'approche globale de l'évaluation d'impact, en conseillant notamment de les utiliser dès le début afin de pouvoir recueillir les points de vue des personnes affectées par le projet sur ce qu'elles considèrent comme important et ce sur quoi doivent porter les mesures. Par ailleurs, il donne des pistes pour conduire des évaluations d'impact dans les cas où la complexité du programme à étudier est importante du fait de la diversité des activités menées dans des secteurs économiques et des aires géographiques différents : ce dernier point pourra être particulièrement utile pour les évaluations d'impact des interventions de REDD+ au niveau infranational et national.

Leeuw, F. et Vaessen, J. 2009 Impact evaluations and development: NONIE guidance on impact evaluation. Banque mondiale, Washington, DC. http://www.worldbank.org/ieg/nonie/ guidance.html.

\section{Rough guide to impact evaluation of environmental and development programs (Pattanayak 2009)}

Ce guide succinct présente les méthodes d'évaluation d'impact expérimentales et quasiexpérimentales dans l'optique d'analyser leur application aux programmes dans les domaines de l'environnement et du développement. L'auteur aborde également les débats actuels relatifs à l'évaluation d'impact variable, en particulier les difficultés posées par l'hétérogénéité des impacts d'un programme selon les sous-groupes de la population considérés. Un exercice d'entraînement est inclus qui consiste à refaire l'évaluation d'impact d'un projet réel à partir de données provenant de l'étude originale.

Pattanayak, S.K. 2009 Rough guide to impact evaluation of environmental and development programs. Document de travail SANDEE 
$\mathrm{n}^{\circ}$ 20-09. Réseau sud-asiatique pour l'économie du développement et de l'environnement (South Asian Network for Development and Environmental Economics), Kathmandou, Népal.

\section{B. Sites Web traitant de l'évaluation d'impact}

Initiative internationale pour l'évaluation d'impact (International Initiative for Impact Evaluation)

(3ie) : http://www.3ieimpact.org/.

Abdul Latif Jameel Poverty Action Lab (J-PAL) :

http://www.povertyactionlab.org/.

Base de connaissances sur les méthodes de recherche (Research Methods Knowledge Base) : http://www.socialresearchmethods.net/kb/ quasiexp.php.

\section{Guides relatifs à I'évaluation participative}

Participatory water monitoring: a guide for preventing and managing conflict (CAO 2008)

La surveillance participative peut aider à développer et à conserver l'appui des populations aux projets locaux. Telle est la leçon principale tirée de l'expérience du médiateur CAO lors de sa mission au sein des communautés affectées par la mine d'or de Newmont/Minera Yanacocha à Cajamarca, au Pérou. Après une fuite de mercure dans la région, la population a exprimé des inquiétudes quant à la pollution de l'eau due à la présence de la mine d'or. Un programme de surveillance de la qualité de l'eau a donc été mis en place dans le but de collecter, d'analyser et de notifier des informations qualitatives et quantitatives sur l'eau avec la participation de la population locale. Ce guide fournit des conseils pour développer un processus de surveillance participative qui pourrait être adapté aux projets de REDD+. Il met en évidence le rôle potentiel de la surveillance participative en tant qu'instrument de cristallisation de la participation de citoyens informés et en nombre suffisant dans les projets et en tant que moyen de consolidation de l'adhésion des communautés locales.

Compliance Advisor Ombudsman (CAO). 2008 Participatory water monitoring: a guide for preventing and managing conflict. CAO, Washington, DC. http://www.cao-ombudsman. org/howwework/advisor/documents/ watermoneng.pdf.
Participatory impact assessment: a guide for practitioners (Catley et al. 2007)

L' " étude d'impact participative » (EIP) telle quelle est définie par ces auteurs " prolonge l'évaluation participative en milieu rural (EPMR) en adaptant les instruments participatifs et en les associant à des approches statistiques classiques dans le but spécifique de mesurer l'impact des projets d'aide humanitaire et de développement sur les vies humaines ». Compte tenu du fait que des informations antérieures au projet et des données de contrôle sont difficiles à obtenir dans bien des cas, ce guide propose d'autres méthodes, de nature participative, qui permettent de mettre en évidence les changements relatifs au bien-être et de les imputer aux activités des projets. Ces méthodes consistent à utiliser une évaluation par la notation et le classement de facteurs liés au projet ou non. Ce guide présente une démarche d'EIP en huit étapes en proposant des instruments et méthodes pour chaque étape.

Catley, A., Burns, J., Adebe, D. et Suji, O. 2007 Participatory impact assessment: a guide for practitioners. Feinstein International Center, Tufts University, Medford, Massachusetts, États-Unis. http://www.reliefweb.int/rw/lib.nsf/db900SID/ SHIG-7L2K8C?OpenDocument.

\section{Most significant change (Davies et Dart 2005)}

Consulter la Section 2.D.

\section{Guides d'évaluation particulièrement adaptés aux interventions dans le domaine de la conservation}

Design alternatives for evaluating the impact of conservation projects (Margoluis et al. 2009)

Cet article présente une synthèse des méthodologies de recherche possibles dans le cadre des évaluations d'actions de conservation. Il résume les approches existantes, quasi-expérimentales, non expérimentales et qualitatives, les informations produites par ces différentes méthodes, ainsi que leurs avantages et inconvénients respectifs. Les auteurs exposent ensuite les spécificités des interventions de conservation qui posent problème pour la définition d'une méthodologie d'évaluation, tout en proposant des stratégies pour surmonter ces obstacles. Ils définissent les méthodologies adaptées à toute une gamme de circonstances et d'interventions dans le domaine de la conservation. 
Margoluis, R., Stem, C., Salafsky, N. et Brown, M. 2009 Design alternatives for evaluating the impact of conservation projects, New Directions for Evaluation, (122): 85-96.

\section{Manual for social impact assessment of land- based carbon projects (Richards et Panfil 2010)} Les Standards de l'Alliance Climat, Communauté et Biodiversité (CCBA) exigent que les projets de carbone forestier fassent la preuve de leurs impacts nets positifs sur les communautés locales. Pour ce faire, les projets doivent : (1) décrire les conditions socioéconomiques des populations existant au moment du lancement du projet ; (2) définir un scénario socioéconomique contrefactuel (« en l'absence du projet »); (3) estimer les conditions socioéconomiques après le projet ; (4) expliquer comment le projet pourra améliorer les conditions socioéconomiques; (5) mettre en place un système de surveillance des impacts sociaux. Jusquà une date récente, le CCBA n'avait pas fourni de directives aux initiateurs de projet sur la manière de mettre en œuvre ces cinq étapes et de faire la démonstration d'impacts nets positifs lors de la validation du projet. Ce manuel permet donc de pallier cette lacune. Il met l'accent sur (1) une approche de modélisation de la causalité pour analyser l'attribution des causes, plutôt que sur la collecte de données relatives à des sites de contrôle et sur (2) l'emploi d'une variante du Cadre des moyens d'existence durables pour étudier les résultats sur le plan du bien-être économique.

Richards, M. et Panfil, S. 2010 Manual for social impact assessment of land-based carbon projects. Version 1.0. Forest Trends, Alliance Climat, Communauté et Biodiversité, Fauna et Flora International et Rainforest Alliance, Washington, DC. http://www.forest-trends.org/documents/ files/doc_2436.pdf.

\section{Social assessment of protected areas: a review of} rapid methodologies (Schreckenberg et al. 2010) Cette revue de littérature très complète synthétise environ 30 instruments et méthodes susceptibles d'être intéressants pour mieux connaitre les impacts sociaux des projets de conservation, en les caractérisant du point de vue de leurs points forts et leurs faiblesses et de leurs cadres théoriques. Cette synthèse permet de relever et de répertorier 200 indicateurs. Pour les auteurs, il existe des lacunes dans les cadres théoriques actuels et les indicateurs étudiés. Ils proposent en outre d'apporter de légères modifications au Cadre des moyens d'existence durables habituellement utilisé, et d'adopter ce nouveau texte comme cadre théorique pour l'analyse des impacts sociaux dans les aires protégées.

Schreckenberg, K., Camargo, I., Withnall, K., Corrigan, C., Franks, P., Roe, D., Scherl, L.M. et Richardson, V. 2010 Social assessment of protected areas: a review of rapid methodologies. Rapport pour l'Initiative d'évaluation sociale des aires protégées (Social Assessment of Protected Areas Initiative - SAPA). International Institute for Environment and Development, Londres.

\section{Household surveys - a tool for conservation design, action and monitoring (WCS 2006)} Ce manuel technique compact fournit des recommandations pour la conception d'une étude des résultats sur le plan du bien-être en utilisant la méthode " avant-après/contrôle-intervention " (BACI), dans laquelle des sites de contrôle et d'intervention sont pré-appariés pour neutraliser les effets de facteurs de confusion potentiels. Il suggère donc les variables à utiliser pour apparier les sites de contrôle et d'intervention, et les variables de confusion potentielles pour lesquelles il est important de collecter des données. Il formule des recommandations concernant l'utilisation d'approches différentes pour analyser le bienêtre des ménages, dont l'Enquête modifiée sur les besoins fondamentaux, la collecte de données sur les revenus et la consommation en espèces, et des mesures biologiques du bien-être (telles que la circonférence du haut du bras pour détecter la malnutrition). De plus, ce manuel propose des techniques d'atténuation des biais d'analyse et de présentation des données, dont l'utilisation des rations caloriques recommandées en fonction de l'âge et du sexe pour estimer le nombre des « équivalents adultes masculins » par ménage, la conversion du revenu à l'aide de la parité du pouvoir d'achat, et l'établissement d'un indice local des prix à la consommation. Il fournit également des conseils en matière de cartographie participative et des techniques de télédétection pour mieux comprendre comment les populations utilisent les ressources naturelles. 
Wildlife Conservation Society (WCS). 2006 Household surveys: a tool for conservation design, action and monitoring. Manuel technique $n^{\circ} 4$. Programme des paysages vivants de la Wildlife Conservation Society, Bronx, New York. http://wcslivinglandscapes.com/ landscapes/90119/bulletins/manuals.html.

\section{E. Etudes illustrant l'application des techniques d'évaluation d'impact aux interventions dans le domaine de la conservation}

Andam, K.S., Ferraro, P.J. et Holland, M.B. 2009 What are the social impacts of land use restrictions on local communities? Empirical evidence from Costa Rica. Communication présentée au Congrès de l'Association internationale des économistes agricoles, Beijing, Chine, 16 au 22 août 2009.

Andam, K.S., Ferraro, P.J., Pfaff, A., SanchezAzofeifa, G.A. et Robalino, J.A. 2008 Measuring the effectiveness of protected area networks in reducing deforestation. Actes de l'Académie Nationale des Sciences des États-Unis, 105(42): 16089-16094.

Andam, K.S., Ferraro, P.J., Sims, K.R.E., Healy, A. et Holland, M.B. 2010. Protected areas reduced poverty in Costa Rica and Thailand. Actes de l'Académie Nationale des Sciences des États-Unis, 107(22): 9996-10001.

Arriagada, R.A. 2008 Private provision of public goods: applying matching methods to evaluate payments for ecosystem services in Costa Rica. Thèse de PhD. Université de l'État de Caroline du Nord, Raleigh, Caroline du Nord, États-Unis.

Bandyopadhyay, S. et Tembo, G. 2009 Household welfare and natural resource management around national parks in Zambia. Document de travail consacré à la recherche sur les politiques $n^{\circ} 4932$. Banque mondiale, Washington, DC.

Gaveau, D.L.A., Wandono, H. et Setiabudi, F. 2007 Three decades of deforestation in southwest Sumatra: have protected areas halted forest loss and logging, and promoted regrowth? Biological Conservation, 134: 495-504.

Jagger, P. 2008 Forest incomes after Uganda's forest sector reform: are the rural poor gaining? Programme du CGIAR à l'échelle du système sur l'action collective et les droits de propriété (CAPRi). Document de travail ${ }^{\circ} 92$. Institut International de Recherche sur les Politiques Alimentaires, Washington, DC.
Jindal, R. 2010 Livelihood impacts of payments for forestry carbon services: field evidence from Mozambique. Dans : Tacconi, L. Mahanty, S. et Suich, H. (éd.) Livelihoods in the REDD? Payments for environmental services, forest conservation and climate change. Edward Elgar, Cheltenham, Royaume-Uni.

Jumbe, C. et Angelsen, A. 2006 Do the poor benefit from devolution policies? Evidence from forest co-management in Malawi. Land Economics, 82(4): 562-581.

Sims, K.R.E. 2010 Conservation and development: evidence from Thai protected areas. Journal of Environmental Economics and Management, 60(2): 94-114.

Somanathan, E., Prabhakar, R. et Mehta, B.S. 2009 Decentralization for cost-effective conservation. Actes de l'Académie Nationale des Sciences des États-Unis, 106(11): 4143-4147.

\section{Mesure des résultats : bien- être économique, physique et psychologique}

\section{A. Instruments et méthodes fondés sur la mesure de biens et de l'accès à des services (biens et services définis localement, indicateurs subjectifs)}

Basic Necessities Survey (Davies 1997) et
Modified Basic Necessities Survey (WCS) L'enquête sur les besoins fondamentaux (BNS) élaborée par Rick Davies en 1997 propose une définition consensuelle et démocratique de la pauvreté, dans laquelle la population étudiée participerait à la détermination des indicateurs de bien-être et à la définition de la pauvreté. Pour ce faire, l'enquête BNS interroge des informateurs principaux et d'autres personnes dans le but de dresser une liste de 20 à 30 besoins fondamentaux : ce sont les biens, les activités et les services auxquels « tout le monde devrait avoir accès et qui ne devraient manquer à personne ». Ces besoins fondamentaux comprennent par exemple une bicyclette, un quart d'hectare de terre à cultiver, trois repas par jour et l'accès à la scolarité. Chaque communauté définit ainsi une liste qui lui est propre. Les évaluateurs interrogent ensuite les ménages pour leur demander si, à leur avis, chaque élément de la liste correspond vraiment à un 
besoin fondamental et s'ils possèdent cet élément. Les éléments qui n’ont pas été retenus par au moins $50 \%$ des personnes interrogées sont rayés de la liste des besoins fondamentaux. Le bien-être de chaque ménage est ensuite mesuré par leur possession ou non possession de chaque élément essentiel de la liste, auquel on affecte un coefficient de pondération correspondant au pourcentage de ménages ayant jugé cet élément essentiel.

L’enquête BNS a été utilisée par Action Aid au Vietnam et par d'autres évaluateurs au Mali et en Ouganda. La Wildlife Conservation Society (WCS) a élaboré une enquête BNS modifiée pour comprendre l'effet des aires protégées sur la subsistance des populations au Gabon, au Guatemala et au Cambodge.

Site Internet de l'enquête BNS de Rick Davies : http://mande.co.uk/special-issues/the-basicnecessities-survey/

Davies, R. 1997 Beyond wealth ranking: the democratic definition and measurement of poverty. http://www.mande.co.uk/docs/democrat.htm.

Pro Poor Center et Davies, R. 2006 The 2006 Basic Necessities Survey (BNS) in Can Loc District, Ha Tinh Province, Vietnam. http://mande.co.uk/ special-issues/the-basic-necessities-survey.

Wildlife Conservation Society (WCS). 2006 Household surveys: a tool for conservation design, action and monitoring. Manuel technique $\mathrm{n}^{\circ} 4$. Programme des paysages vivants de la Wildlife Conservation Society, Bronx, New York. http:// wcslivinglandscapes.com/landscapes/90119/ bulletins/manuals.html (consulté en novembre 2010).

Wildlife Conservation Society [sans date] Assessing the impact of conservation and development on rural livelihoods: using a modified basic necessities survey in experimental and control communities. Programme des paysages vivants de la Wildlife Conservation Society, Bronx, New York.

\section{Stages of progress (Krishna 2005)}

Développée par Anirudh Krishna à l'université Duke aux États-Unis, la méthode des étapes du progrès s'attache à appréhender la pauvreté du point de vue des plus démunis eux-mêmes et à lever le voile sur les causes des entrées et sorties de pauvreté des ménages. Cette méthode a été utilisée auprès de milliers de ménages en Inde, au Kenya, au Pérou, en Ouganda et aux États-Unis. La première étape de cette démarche consiste à organiser une réunion avec la population dans le but de convenir d'une définition de la " pauvreté » (ne pas avoir assez à manger par ex.) et de ce qui distingue les pauvres et les très pauvres des autres classes économiques, c'est-à-dire les étapes à franchir pour sortir de la pauvreté (acheter un toit en tôle, une chèvre, une mobylette ou une voiture, envoyer un enfant à l'école, payer ses dettes, etc.). Les enquêteurs présentent ensuite au groupe la liste de tous les ménages du village et un événement mémorable marquant dans le passé ou l'année précédant le projet (élection ou sécheresse par ex.) pour lui demander de caractériser les entrées et sorties de pauvreté de chaque ménage sur la période considérée selon les critères suivants : ménage (1) demeuré pauvre, (2) sorti de la pauvreté, (3) devenu pauvre, (4) demeuré non pauvre. Les enquêteurs établissent les causes de la variation ou de la stabilité des conditions économiques des ménages en interrogeant un échantillon aléatoire de ménages provenant des quatre groupes lors d'entretiens collectifs et individuels.

Stages of Progress: disaggregating poverty for better policy impact. Site internet. http://sanford.duke. edu/krishna/.

Krishna, A. 2005 Stages of Progress field manual: a community based methodology for defining and understanding poverty. Version 2.0. http:// sanford.duke.edu/krishna/SoP.pdf.

\section{Sustainable Livelihoods Framework (Cadre des moyens d'existence durables)}

Le Cadre des moyens d'existence durables (CMED) initial (également connu sous le nom d'Approche des moyens d'existence durables ou AMED) a pour objet la mesure de la possession de cinq catégories de capital : humain (santé, éducation par ex.), social (réseaux, institutions officielles et officieuses par ex.), physique (infrastructure, outils par ex.), financier (revenu, économies, crédit par ex.) et naturel (produits de la forêt, terrains, eau par ex.). Le CMED a également été utilisé pour analyser le bien-être au niveau d'une communauté donnée. Des indicateurs (qui pourraient être définis localement) sont utilisés pour mesurer le capital d'un ménage (d'un individu ou d'une communauté) dans chacune des cinq catégories. Les valeurs des indicateurs produisent le pentagone unique de chaque ménage, individu ou communauté. Le CMED comprend également 
l'analyse des vulnérabilités et des chocs principaux affectant les moyens d'existence. Selon le CMED, les moyens de subsistance ne sont pas durables s'ils sont menacés par ces vulnérabilités de telle sorte qu'ils ne peuvent suffir à alimenter, voire à augmenter les cinq catégories de capital sans nuire aux ressources naturelles.

Depuis sa conception dans les années 90, le CMED a été utilisé et modifié par de nombreux organismes de développement et ONG ; à l'heure actuelle, il figure dans les référentiels de certification relatifs au carbone forestier. Par exemple, la Méthode d'évaluation des situations paysagères (LOAM) développée par le WWF définit une sixième catégorie de bien-être : les biens mondiaux pour la conservation. La norme du carbone social utilise un CMED modifié appelé « méthodologie du carbone social » qui repose sur six catégories de capital : naturel, financier, humain, social, lié au carbone, lié à la biodiversité. Plus récemment, l'initiative pour l'évaluation sociale des aires protégées (SAPA) a proposé d'intégrer le cadre d'analyse des écosystèmes du millénaire dans le CMED de manière à subdiviser le capital naturel selon qu'il s'agisse de l'offre, de l'appui ou de la régulation des services écosystémiques et d'élargir le capital social aux services culturels des écosystèmes. La SAPA comprend aussi une sixième catégorie : le capital politico-légal, lié aux droits de l'homme et à l'inclusion.

\section{Références principales}

Aldrich, M. et Sayer, J. 2007 In practice: landscape outcomes assessment methodology (LOAM). Programme Des forêts pour la vie duWWF (WWW Forests for Life Programme). http://assets.panda.org/downloads/ loaminpracticemay07.pdf.

Carney, D. (éd.) 1998 Sustainable rural livelihoods: what contribution can we make? Department For International Development (DFID), Londres.

Chambers, R. et Conway, G. 1992 Sustainable rural livelihoods: practical concepts for the 21st century. Institute of Development Studies, Brighton, Royaume-Uni.

Site Internet de l'Approche des moyens d'existence durables du Fonds International de Développement Agricole (FIDA) : http://www. ifad.org/sla/index.htm.

Sayer, J., Campbell, B., Petheram, L., Aldrich, M., Ruiz Perez, M., Endamana, D., Nzooh Dongmo,
Z.-L., Defo, L., Mariki. S., Doggart, N. et Burgess, N. 2007 Assessing environment and development outcomes in conservation landscapes.

Biodiversity Conservation, 16(9): 2677-2694.

Schreckenberg, K., Camargo, I., Withnall, K., Corrigan, C., Franks, P., Roe, D., Scherl, L.M. et Richardson, V. 2010 Social assessment of protected areas: a review of rapid methodologies. Rapport pour l'Initiative d'évaluation sociale des aires protégées (Social Assessment of Protected Areas Initiative). International Institute for Environment and Development, Londres. Site Internet de Social Carbon Methodology : www.socialcarbon.org.

\section{B. Instruments/méthodes reposant sur la mesure du revenu et de la consommation (indicateurs objectifs prédéfinis)}

World Bank Living Standards Measurement Study Surveys (Banque mondiale 1980-en cours)

Lancée par la Banque mondiale, l'Étude de mesure des niveaux de vie (LSMS) a pour objectif d'améliorer la collecte de données par les organismes de statistiques nationaux et d'augmenter la prise en compte des données liées aux ménages dans les processus décisionnels relatifs aux politiques de développement. Les enquêtes collectent des données au niveau du ménage sur différents aspects liés au bien-être, dont la consommation, le revenu, l'emploi, le niveau d'éducation et des mesures physiques de la santé humaine, mais aussi des informations sur la migration et la fertilité. Les enquêtes auprès des ménages sont complétées par un questionnaire destiné à la communauté dans son ensemble (entretiens avec les représentants de celle-ci) et un questionnaire sur les prix (entretiens avec des vendeurs sur les marchés pour se renseigner sur les prix). Des enquêtes LSMS pilotes ont été réalisées en Côte d'Ivoire et au Pérou en 1985. Depuis, les enquêtes ont été réalisées dans plusieurs autres pays. La phase actuelle de l'étude LSMS (20082015) a pour objet d'acquérir des connaissances sur l'agriculture et les liens existant entre les activités agricoles et non agricoles en Afrique, dans le but de générer des séries de données de panel nationales représentatives. Les données des enquêtes terminées et certains des instruments analytiques utilisés sont disponibles sur le site Web LSMS de la Banque mondiale. Ces séries de données pourraient être intéressantes dans le cadre de l'étude des 
impacts de la REDD+ au plan national, malgré une disponibilité des données limitée à certains des pays destinés à recevoir la REDD+.

Disponible sur le site de la Banque mondiale http:// econ.worldbank.org/WBSITE/EXTERNAL/ EXTDEC/EXTRESEARCH/EXTLSMS/0,,content MDK:21610833 pagePK:64168427 piPK:641684 35 theSitePK:3358997,00.html.

\section{Poverty Environment Network (PEN) du CIFOR}

Le Réseau pauvreté et environnement est une initiative de collaboration à laquelle participent des chercheurs en doctorat et des chercheurs débutants des pays en développement. Des questionnaires communs destinés aux villages et ménages et portant sur le revenu tiré de la forêt et de l'environnement ont été employés dans plus de 35 sites de recherche dans les régions des tropiques où le revenu est faible. L'objectif de ce projet est non seulement de recueillir des informations sur le revenu tiré de la forêt et de l'environnement, mais aussi de mieux comprendre les rapports complexes entre la réduction de la pauvreté et la dépendance aux forêts. Des données relatives à plus de 8000 ménages ont été collectées entre 2003 et 2009. www.cifor.cgiar.org/pen

\section{Instruments/méthodes fondés sur les droits, la sécurité des conditions d'existence et les opportunités}

\section{The 'BAG': Basic Assessment Guide for Human Well-Being (Colfer et al. 1999)}

Le «BAG» (Guide de l'évaluation de base) a été élaboré en 1999 par Carol Colfer et des collaborateurs du CIFOR pour analyser la « durabilité » des activités de la filière bois, mais il peut également être adapté pour comprendre les impacts de la REDD+. Ce guide a pour objet l'analyse des effets des activités de production de bois sur les populations locales. Associé à deux autres boîtes à outils du CIFOR centrées sur le fonctionnement écologique et les effets de l'exploitation forestière, il forme un outil de travail en trois parties qui permet d'analyser les activités de la filière bois du point de vue de leur "durabilité », celle-ci étant définie comme étant «le maintien ou l'amélioration du bien-être humain et du fonctionnement écologique ». Le «BAG» pose les trois principes suivants : (1) la gestion des forêts préserve ou améliore l'accès équitable de toutes les générations aux ressources et aux bénéfices économiques ; (2) les parties prenantes concernées jouissent de droits et de moyens reconnus pour gérer les forêts dans la coopération et le respect de l'équité ; et (3) la santé des intervenants dans la forêt, l'état de la culture et de la forêt elle-même sont acceptables pour toutes les parties prenantes. Afin d'évaluer la conformité à chacun de ces principes, le «BAG» définit des critères et des indicateurs utiles pour l'évaluation, ainsi que des instruments. Il préconise également l'utilisation d'un tableau des acteurs dont le bien-être est tributaire de la forêt (Who Counts Matrix), et le recours à des réunions de consultation pour dresser la liste des parties prenantes dès le début du projet. Ces instruments guident également les utilisateurs dans le processus de notation des principes selon une échelle à 10 points, chacun des principes étant affecté d'un coefficient reflétant son importance relative.

Le « BAG » est accompagné de deux instruments complémentaires : (1) Le « sac à surprises »(Grab Bag) qui présente des méthodes complémentaires et (2) le " Guide de notation et d'analyse » (The Scoring and Analysis Guide) qui explicite la systématisation des jugements qualitatifs et l'application de méthodes simplifiées d'analyse de données quantitatives en utilisant Excel et SPSS.

D'autres boîtes à outils du CIFOR sont disponibles sur : http://www.cifor.cgiar.org/acm/methods/ toolbox.html.

Colfer, C.J.P., Brocklesby, M.A., Diaw, C., Etuge, P., Günter, M., Harwell, E., McDougall, C., Porro, N.M., Porro, R., Prabhu, R. et al. 1999 The BAG: basic assessment guide for human well-being. Manuel de critères et indicateurs $n^{\circ} 5$. CIFOR, Bogor, Indonésie.

Colfer, C.J.P., Brocklesby, M.A., Diaw, C., Etuge, P., Günter, M., Harwell, E., McDougall, C., Porro, N.M., Porro, R., Prabhu, R. et al. 1999 The Grab Bag: supplementary methods for assessing human well-being. Manuel de critères et indicateurs $n^{\circ} 6$. CIFOR, Bogor, Indonésie.

Salim, A., Colfer, C.J.P. et McDougall, C. 1999 Guide de notation et d'analyse pour l'évaluation du bien-être social. Manuel de critères et indicateurs $n^{\circ} 7$. CIFOR, Bogor, Indonésie. 


\section{Household livelihood security assessments: a toolkit for practitioners (CARE 2002)}

Selon les auteurs, les analyses de la sécurité des moyens d'existence des ménages (Household livelihood security assessments ou HLSA) constituent un outil d'évaluation rapide ou d'évaluation participative en milieu rural. L'approche, fondée sur les droits, s'appuie sur une analyse pluridisciplinaire pour « mieux appréhender les systèmes relatifs aux moyens de subsistance locaux ... et les différences importantes existant entre les catégories de ménages et entre les individus au sein d'un même ménage ». Cette évaluation décompose les données par groupe (origine ethnique, appartenance hommesfemmes, statut économique ou social, âge etc.) dans le but de comprendre les différences au regard de certains critères : accès aux biens et services, degré de contrôle exercé sur les ressources, division du travail, exercice des droits, accumulation de capital, vulnérabilité et marginalisation et répartition du pouvoir politique et économique. Dans ce manuel, au tableau des utilisations possibles de la boîte à outils HLSA ne figure pas l'évaluation des impacts de projet/programme (l'accent est plutôt mis sur l'étude des conditions existant avant et pendant le projet et sur le développement d'un appui suffisant aux projets CARE). Cependant, l'utilisation des méthodes de ce manuel pourrait souvent être utile pour des évaluations d'impact à bas coût dans le cadre de la REDD+, en particulier si elles portent sur les problèmes de la sécurité alimentaire, de la vulnérabilité et de la marginalisation.

Ce manuel présente les étapes associées aux phases de pré-évaluation, évaluation et analyse en proposant à chaque fois de nombreux outils. La préévaluation concerne l'étude de données secondaires, le repérage des groupes vulnérables et la création de profils de sécurité du point de vue de la subsistance. La phase d'évaluation consiste à recueillir des données qualitatives et quantitatives sur les systèmes de subsistance et le bien-être économique, ainsi que des données relatives aux causes et à leurs effets (facteurs conduisant à la vulnérabilité par ex.). Ce manuel propose également de trianguler les méthodes de collecte des données : enquêtes auprès des ménages, groupes de consultation, entretiens avec des informateurs principaux, classement en fonction du niveau de richesse, représentation participative des liens de causes à effets, etc. Il fournit des recommandations en ce qui concerne les stratégies d'échantillonnage aléatoires et systématiques, et la sélection et la formation du personnel chargé des enquêtes. De plus, cet ouvrage présente différentes méthodes d'analyse des données : analyses d'opportunités, des problématiques hommes-femmes, des institutions et analyses des bénéfices et préjudices.

\section{CARE. 2002 Household livelihood security} assessments: a toolkit for practitioners. Préparé pour l'unité PHLS par TANGO International Inc. http://www.proventionconsortium.org/themes/ default/pdfs/CRA/HLSA2002_meth.pdf.

\section{Instruments/méthodes convenant pour les analyses rétrospectives}

\section{Strengthening the evaluation of programme effectiveness through reconstructing baseline data (Bamberger 2009)}

Pour répondre au problème récurrent du manque de données relatives à l'avant-projet dans les évaluations d'impact, Bamberger présente diverses techniques quantitatives, qualitatives et mixtes qui peuvent permettre à l'évaluateur de " reconstituer » les données antérieures au projet (niveau de référence). Les méthodes abordées comprennent l'utilisation de données secondaires telles que les enquêtes de la Banque mondiale dans le cadre de l'Étude de mesure des niveaux de vie (LSMS) (consulter le paragraphe correspondant dans la Section II(B)), la méthode de remémoration, ou des entretiens avec des informateurs principaux ou des groupes de consultation. Cet article présente également des méthodes pour la reconstitution de données initiales pour le groupe de comparaison (contrôle) : appariement par score de propension, appariement libre, groupe de comparaison des bénéficiaires en attente de projet (pipeline comparison group), groupe de comparaison interne et analyse du score d'intensité, méthodologie transversale du test de vérification des groupes de projet et de comparaison et analyse typologique.

Bamberger, M. 2009 Strengthening the evaluation of programme effectiveness through reconstructing baseline data. Journal of Development Effectiveness, 1(1): 37-59. 


\section{Most significant change (Davies et Dart 2005)}

La technique du " changement le plus significatif » est une méthode participative qualitative qui peut convenir dans le cas où les ressources sont limitées et quand il n'existe pas de données antérieures au projet. Cette technique consiste à recueillir des témoignages de "changement le plus significatif " en employant plusieurs méthodes : les évaluateurs inscrivent des récits spontanés qu'ils ont entendu, conduisent et transcrivent des entretiens, animent et prennent en note des discussions de groupe et/ ou demandent à des personnes d'écrire leur version de faits qui leur semblent importants. Les comptes rendus les plus «significatifs » sont choisis de la manière suivante : tout d'abord, les participants lisent leurs témoignages en groupe, ensuite le groupe délibère sur les témoignages à retenir avant de décider quels sont les témoignages les «plus significatifs » (en procédant à un vote, en attribuant une note aux témoignages publiquement, ou par vote réitératif). Enfin, les raisons qui motivent le choix du groupe sont notées. Après ces étapes participatives, ont lieu les étapes de « vérification des témoignages », quantification, analyse secondaire, et méta-surveillance.

\section{Références principales}

Davies, R. et Dart, J. 2005 Most significant change (MSC) technique: a guide to its use. http://mande. co.uk/docs/MSCGuide.pdf.

Site Internet de Rick Davies sur le changement le plus significatif : http://mande.co.uk/specialissues/most-significant-change-msc/.

Site Internet de l'UNICEF Inde sur le changement le plus significatif : http://www. mostsignificantchange.org/.

\section{Stages of progress (Krishna 2005) \\ Cf. Section 2.A.}

Participatory impact assessment: a guide for practitioners (Catley et al. 2007)

Cf. Section 1.C.

\section{Recueil d'opinions sur les utilisations des sols et leur évolution}

Programme international de recherche sur les
institutions et les ressources forestières (IFRI)

Le Programme international de recherche sur les institutions et les ressources forestières
(IFRI) recueille des données sur la gouvernance et les institutions liées aux forêts depuis 1992. Ces ressources fournissent un excellent point de départ pour l'élaboration de questionnaires portant sur les institutions locales, l'action collective et l'état des forêts. Les protocoles de l'IFRI contiennent des recommandations sur les procédures d'échantillonnage de placettes forestières représentatives et sur le recueil de données biophysiques sur l'état des forêts et leur dégradation. L'IFRI comprend également des analyses qualitatives de la déforestation et de la dégradation des forêts par des groupes d'acteurs allant de villageois aux agents de l'administration des forêts.

\section{Autres sources}

Caviglia-Harris, J.L. et Harris, D.W. 2005

Examining the reliability of survey data with remote sensing and geographic information systems to improve deforestation modeling. Review of Regional Studies, 35(187-205).

Caviglia-Harris, J.L. et Harris, D.W. 2008 Integrating survey and remote sensing data to analyze land use at a fine scale: insights from agricultural households in the Brazilian Amazon. International Regional Science Review, 31: 115-137.

Kerr, J. et Pender, J. 2005 Farmers' perceptions of soil erosion and its consequences in India's semiarid tropics. Land Degradation and Development, 16: 257-271.

Ostrom, E. et Wertime, M.B. 2000 International forestry resources and institutions research strategy. Dans: Gibson, C., McKean, M. et Ostrom, E. (éd.) People and forests: communities, institutions, and governance, p. 1-28. MIT Press, Cambridge, Massachusetts, États-Unis.

Cartographie participative (Centre international pour la recherche agricole orientée vers le développement). http://www.icra-edu.org/ objects/anglolearn/Maps_\&_transectsGuidelines.pdf.

Potvin, C., Tschakert, P., Lebel, F., Kirby, K., Barrios, H., Bocariza, J., Caisamo, J., Caisamo, L., Cansari, C., Casamá, J., et al. 2007 A participatory approach to the establishment of a baseline scenario for a reforestation Clean Development Mechanism project. Mitigation and Adaptation Strategies for Global Change, 12:1341-1362 


\section{Moteurs de la déforestation et de la dégradation}

La déforestation et la dégradation se produisent sous l'effet d'un écheveau complexe de forces de nature économique et institutionnelle. Les moteurs des changements d'utilisation des sols varient selon les paysages, évoluent au fil du temps et sont souvent difficiles à cerner. Les causes de la déforestation et de la dégradation ont donc fait l'objet d'importantes recherches et suscité de vifs débats. Or, il est nécessaire de comprendre ces causes dans un paysage donné si l'on veut concevoir des interventions efficaces dans le domaine de la conservation et formuler des théories du changement dans le cadre des projets et de l'évaluation d'impact. Cette section fournit une liste des études récentes portant sur les moteurs de la déforestation et dégradation, ainsi qu'une courte sélection de la littérature consacrée aux moteurs dans certaines des grandes régions de mise en œuvre de la REDD+.

\section{A. Synthèses et études}

Angelsen, A. et Kaimowitz, D. 1999 Rethinking the causes of deforestation: lessons from economic models. The World Bank Research Observer, 14(1): 73-98. Banque mondiale, Washington, DC.

Barbier, E.B. 2001 The economics of tropical deforestation and land use: an introduction to the special issue. Land Economics, 77(2): 155-171.

Butler, R.A. et Lawrence, W.F. 2008 New strategies for conserving tropical forests. Trends in Ecology and Evolution, 23(9): 469-472.

Chomitz, K., Balmford, A., Whitten, T., Richards. M. et Berlin, A. 2007 At loggerheads? Agricultural expansion, poverty reduction, and environment in tropical forests. World Bank Policy Research Report. Banque mondiale, Washington, DC. http://go.worldbank.org/TKGHE4IA30.

DeFries, R.S., Rudel, T., Uriarte, M. et Hansen, M. 2010 Deforestation driven by urban population growth and agricultural trade in the twenty-first century. Nature Geoscience, 3: 178-181.

Geist, H.J. et Lambin, D.F. 2002 Proximate causes and underlying driving forces of tropical deforestation. BioScience, 52(2): 143-150.

Kanninen, M., Murdiyarso, D., Seymour, F., Angelsen, A., Wunder, S. et German, L. 2007 Do trees grow on money? The implications of deforestation research for policies to promote REDD. CIFOR, Bogor, Indonésie.
Lawlor, K. 2009 Addressing the causes of tropical deforestation: lessons learned and the implications for international forest carbon policy. Dans : Olander, L.P., Boyd, W., Lawlor, K., Myers Madeira, E. et Niles, J.O. (éd.) International forest carbon and the climate change challenge: issues and options, p. 43-53. Nicholas Institute for Environmental Policy Solutions, Duke University, Durham, Caroline du Nord, États-Unis

\section{B. Amazonie}

Araujo, C., Araujo Bonjean, C., Combes, J.-L., Combes Motel, P. et Reis, E.J. 2009 Property rights and deforestation in the Brazilian Amazon. Ecological Economics, 68: 2461-2468.

Asner, G.P., Knapp, D.E., Broadbent, E.N., Oliveira, P.J.C., Keller, M. et Silva, J.N. 2005 Selective logging in the Brazilian Amazon? Science, 310: 480-482.

McAlpine, C.A., Etter, A., Fearnside, P.M., Seabrook, L. et Laurance, W.F. 2009 Increasing world consumption of beef as a driver of regional and global change: A call for policy action based on evidence from Queensland (Australia), Colombia and Brazil. Global Environmental Change, 19: 21-33.

Morton, D., DeFries, R.S., Shimabukuro, Y.E., Anderson, L.O., Arai, E., Bon Espirito-Santo, F., Freitas, R. et Morisette, J. 2006 Cropland expansion changes deforestation dynamics in the southern Brazilian Amazon. Actes de l'Académie Nationale des Sciences des États-Unis, 103(39): 14637-14641.

Pfaff, A. 1999 What drives deforestation in the Brazilian Amazon? Evidence from satellite and socioeconomic data. Journal of Environmental Economics and Management, 37(1):26-43.

\section{Amérique centrale}

Barbier, E.B. et Burgess, J.C. 1996. Economic analysis of deforestation in Mexico. Environment and Development Economics, 1: 203-239.

Kaimowitz, D. 1996 Livestock and deforestation in Central America in the 1980s and 1990s: a policy perspective. CIFOR, Bogor, Indonésie.

\section{Asie du Sud-Est}

Angelsen, A. 1995 Shifting cultivation and 'deforestation': a study from Indonesia. World Development, 23(10): 1713-1729. 
Barbier, E.B., Bockstael, N., Burgess, J.C. et Strand, I. 1995 The linkages between the timber trade and tropical deforestation - Indonesia. World Economy, 18(3): 411-442.

Curran, L.M., Trigg, S.N., McDonald, A.K., Astiani, D., Hardiono, Y.M., Siregar, P., Caniago, I. et Kasischke, E. 2004 Lowland forest loss in protected areas of Indonesian Borneo. Science, 303(5660): 1000-1003.

Fitzherbert, E.B., Struebig, M.J., Morel, A., Danielsen, F., Bruhl, C.A., Donald, P.F. et Phalan, B. 2008 How will oil palm expansion affect biodiversity? Trends in Ecology and Evolution, 23(10): 538-545.

Global Witness 2007 Cambodia's family trees: illegal logging and the stripping of public assets by Cambodia's elite. Global Witness, Londres.

Palmer, C. 2001 The extent and causes of illegal logging: an analysis of a major cause of deforestation in Indonesia. Document de travail du CSERGE. Centre for Social and Economic Research on the Global Environment (CSERGE), Londres.

Sunderlin, W.D. et Resosudarmo, I.A.P. 1996 Rates and causes of deforestation in Indonesia: towards a resolution of the ambiguities. Document occasionnel $n^{\circ}$ 9. CIFOR, Bogor, Indonésie.

\section{E. Asie du Sud}

Bajracharya, D. 1983 Deforestation in the food/fuel context: historical and political perspectives from Nepal. Mountain Research and Development, 3(3): 227-240.

Lele, N., Nagendra, H. et Southworth, J. 2010 Accessibility, demography and protection: drivers of forest stability and change at multiple scales in the Cauvery Basin, India. Remote Sensing, (2): 306-332.

\section{F. Bassin du Congo}

De Wasseige, C., Devers, D., de Marcken, P., Eba’a Atyi, R., Nasi, R. et Mayaux, P. (éd.) 2009 The forests of the Congo Basin: state of the forest 2008. Office des publications de l'Union européenne, Luxembourg. http://carpe.umd.edu/ resources/sof/.

Hansen, C.P. et Treue, T. 2008 Assessing illegal logging in Ghana. International Forestry Review, 10(4): 573-590.

Jenkins, M. 2008 Who murdered the Virunga gorillas? National Geographic, juillet, p. 34-65.
Laporte, N.T., Stabach, J.A., Grosch, R., Lin, T.S. et Goetz, S.J. 2007 Expansion of industrial logging in Central Africa. Science, 316(5830): 1451.

Mertens, B., Sunderlin, W.D., Ndoye, O. et Lambin, E.F. 2000 Impact of macroeconomic change on deforestation in south Cameroon: integration of household survey and remotely-sensed data. World Development, 28(6): 983-999.

Zhang, Q., Devers, D., Desch, A., Justice, C.O. et Townshend, J. 2005 Mapping tropical deforestation in Central Africa. Environmental Monitoring and Assessment, 101(1-3): 69-83.

\section{G. Afrique occidentale}

Appiah, M., Blay, D., Damnyag, L., Dwomoh, F.K., Pappinen, A. et Luukkanen, O. 2009 Dependence on forest resources and tropical deforestation in Ghana. Environment, Development and Sustainability, 11: 471-487.

Barbier, E.B. et Benhin, J.K.A. 2001 The effects of the structural adjustment program on deforestation in Ghana. Agricultural and Resource Economics Review, 30(1): 66-88.

Fairhead, J. et Leach, M. 1998 Reframing deforestation: global analyses and local realities with studies in West Africa. Routledge, Londres. Global Witness 2005 Timber, Taylor, soldier spy: how Liberia's uncontrolled resource exploitation, Charles Taylor's manipulation and the rerecruitment of ex-combatants are threatening regional peace. Rapport de Global Witness soumis au Conseil de sécurité des Nations Unies.

Hansen, C.P. et Treue, T. 2008 Assessing illegal logging in Ghana. International Forestry Review, 10(4): 573-590.

\section{Mesure du carbone forestier dans le cadre de la REDD+}

La littérature consacrée au thème parallèle de la mesure des résultats sur le plan du carbone forestier nous intéresse car ce guide s'attache à comprendre les effets de la REDD+ sur les conditions d'existence des populations. La littérature abondante traitant de la définition des niveaux d'émission de référence (NER) relatifs à la déforestation et à la dégradation et des moyens de résolution du problème des fuites dans le cadre des interventions de REDD+, ainsi que celle qui traite de la modélisation des agents et d'autres méthodes de prévision de l'évolution des utilisations des sols peuvent éclairer l'élaboration 
des scénarios contrefactuels liés à l'étude des résultats sociaux et écologiques dans le cadre des évaluations d'impact. De plus, pour analyser les synergies et les arbitrages entre les impacts de la REDD+ sur le carbone forestier et sur le bien-être économique et approfondir nos connaissances sur la production conjointe et les boucles de rétroaction dans des systèmes socio-écologiques complexes, il faudra que les évaluations veillent à ce que les scénarios de référence relatifs à la déforestation et la dégradation ainsi qu'aux aspects sociaux soient établis en tandem. Cette partie fournit un échantillon synthétique de références sur ces thèmes, provenant soit de la littérature scientifique, soit des référentiels de certification.

\section{A. Guides relatifs aux pratiques d'excellence}

GOFC-GOLD 2009 A sourcebook of methods and procedures for monitoring and reporting anthropogenic greenhouse gas emissions and removals caused by deforestation, gains and losses of carbon stocks in forests remaining forests and forestation. http://www.gofc-gold.uni-jena. $\mathrm{de} / \mathrm{redd} /$.

Estrada, M. 2011 Standards and methods available for estimating project-level REDD+ carbon benefits: reference guide for project developers. Document de travail $n^{\circ} 52$. CIFOR, Bogor, Indonésie.

\section{B. Analyses contrefactuelles relatives à la déforestation et la dégradation}

\section{Niveaux d'émission de référence (NER) à l'échelon national : propositions et débats contradictoires}

Angelsen, A. 2008a REDD models and baselines. International Forestry Review 10(3): 465-475.

Angelsen, A. 2008b How do we set the reference levels for REDD payments? Dans : Angelsen, A. (ed.) Moving ahead with REDD: issues, options and implications, 53-64. CIFOR, Bogor, Indonesia.

Angelsen, A. 2008a REDD models and baselines. International Forestry Review, 10(3): 465-475.

Angelsen, A. 2008b How do we set the reference levels for REDD payments? Dans: Angelsen, A. (éd.). Moving ahead with REDD: issues, options and implications. CIFOR, Bogor, Indonésie, p. 53-64.

Griscom, B., Shoch, D., Stanley, B., Cortez, R. et Virgillo, N. 2009 Sensitivity of amounts and distribution of tropical forest carbon credits depending on baseline rules. Environmental Science and Policy, 12(7): 897-911.

Karsenty, A. 2008 The architecture of proposed REDD schemes after Bali: facing critical choices. International Forestry Review, 10(3): 443-457.

Motel, P.C., Pirard, R. et Combes, J.-L. 2008 A methodology to estimate impacts of domestic policies on deforestation: Compensated Successful Efforts for 'avoided deforestation'. Ecological Economics, 68(3): 680-691.

Tacconi, L. 2009 Compensated successful efforts for avoided deforestation vs. compensated reductions. Ecological Economics, 68(8-9): 2469-2472.

\section{NER à l'échelon du projet et à l'échelon infranational : critiques et débats}

Plantinga, A.J. et Richards, K.R. 2008 International forest carbon sequestration in a post-Kyoto agreement. Projet d'Harvard relatif aux accords internationaux sur le climat. Document de travail 2008-11. Cambridge, Massachusetts, États-Unis.

Richards, K. et Andersson, K. 2001 The leaky sink: persistent obstacles to a forest carbon sequestration program based on individual projects. Climate Policy, 1:(41-54).

Schlamadinger, B., Ciccarese, L., Dutschke, M., Fearnside, P.M., Brown, S. et Murdiyarso, D. 2005 Should we include avoidance of deforestation in the international response to climate change? Dans : Murdiyarso, D. et H. Herawati, H. (éd.) Carbon forestry: who will benefit? Actes de l'atelier sur la séquestration du carbone et les moyens de subsistance durables, Bogor, Indonésie, 16 au 17 février. CIFOR, Bogor, Indonésie.

\section{NER à l'échelon du projet et à l'échelon infranational : méthodologies des référentiels de certification}

Voluntary Carbon Standard Guidance and Tools : http://www.v-c-s.org/afl.html.

Voluntary Carbon Standard Methodologies :

- approuvées : http://www.v-c-s.org/ vcsmethodologies.html.

- à l'étude : http://www.v-c-s.org/public_comment. html. 


\section{Approches tirées de la littérature scientifique}

Brown, S., Hall, M., Andrasko, K., Ruiz, F., Marzoli, W., Guerrero, G., Masera, O., Dushku, A., DeJong, B. et Cornell, J. 2007 Baselines for landuse change in the tropics: application to avoided deforestation projects. Mitigation and Adaptation Strategies for Global Change, 123(86): 1001-1026.

Gaveau, D.L.A., Epting, J., Lyne, O., Linkie, M., Kumara, I., Kanninen, M. et Leader-Williams, N. 2009 Evaluating whether protected areas reduce tropical deforestation in Sumatra. Journal of Biogeography, 36(11): 2165-2175.

Honey-Roses, J., Lopez-Garcia, J., Rendon-Salinas, E., Peralta-Higuera, A. et Galindo-Leal, C. 2009 To pay or not to pay? Monitoring performance and enforcing conditionality when paying for forest conservation in Mexico. Environmental Conservation, 36(2): 120-128.

Potvin, C., Tschakert, P., Lebel, F., Kirby, K., Barrios, H., Bocariza, J., Caisamo, J., Caisamo, L., Cansari, C., Casamá, J., et al. 2007 A participatory approach to the establishment of a baseline scenario for a reforestation Clean Development Mechanism project. Mitigation and Adaptation Strategies for Global Change, 12: 1341-1362.

Veldkamp, A. et Lambin, E.F. 2001 Editorial: predicting land-use change. Agriculture, Ecosystems and Environment, 85(1-6).

Veldkamp, A. et Verburg, P.H. 2004 Editorial: modeling land use change and environmental impact. Journal of Environmental Management, 72(1-3).

\section{a. Modélisation des agents}

Evans, T.P. et Kelley, H. 2004 Multi-scale analysis of a household level agent-based model of land cover change. Journal of Environmental Management, 72:57-72.

Castella, J.-C., Kam, S.P., Quang, D.D., Verburg, P.H. et Hoanh, C.H. 2007 Combining top-down and bottom-up modeling approaches of land use/cover change to support public policies: application to sustainable management of natural resources in northern Vietnam. Land Use Policy, 24: 531-545.

Parker, D.C., Manson, S.M., Janssen, M.A., Hoffmann, M.J. et Deadman, P. 2003 Multi-agent systems for the simulation of land-use and landcover change: a review. Annals of the Association of American Geographers, 93(2): 314-337.

Walsh, S.J., Crawford, T.W., Crews-Meyer, K.A. and Welsh, W.F. $2001 \mathrm{~A}$ multiscale analysis of land use land cover change and NDVI variation in Nang Rong district, northeast Thailand. Agriculture, Ecosystems and Environment, 85: 47-64.

\section{Fuites}

\section{Moyens de lutte contre les fuites aux niveaux national et international}

Murray, B.C. 2009 Leakage from an avoided deforestation compensation policy: concepts, empirical evidence, and corrective policy options. Dans : Palmer, C. et Engel, S. (éd.). Avoided deforestation: prospects for mitigating climate change, p. 11-38. Routledge, New York.

Wunder, S. 2008 How do we deal with leakage? Dans : Angelsen, A. (éd.) Moving ahead with REDD: issues, options and implications, p. 65-76. CIFOR, Bogor, Indonésie.

\section{Moyens de lutte contre les fuites au niveau du projet : méthodologies des référentiels de certification}

Voluntary Carbon Standard Guidance and Tools : http://www.v-c-s.org/afl.html.

Voluntary Carbon Standard Methodologies :

- approuvées : http://www.v-c-s.org/ vcsmethodologies.html.

- à l'étude : http://www.v-c-s.org/public_ comment.html. 


\section{Annexe C. À propos des recommandations techniques et des instruments d'enquête}

L'Étude comparative mondiale du CIFOR sur la REDD+ (GCS-REDD+) comprend une évaluation rigoureuse des impacts sociaux et biophysiques des projets pilotes de REDD+. Les documents présentés dans cette annexe forment la pierre angulaire des instruments d'évaluation des impacts sociaux utilisés dans cette étude. Les questionnaires et recommandations techniques qui accompagnent ces documents relèvent du domaine public international. A ce titre, ils sont à la disposition du personnel des organismes scientifiques, bailleurs de fond, organisations non-gouvernementales et de la société civile, organismes de conservation et des communautés d'usagers des forêts. Il s'agit de plusieurs questionnaires créés pour obtenir des informations : leur structure imbriquée permet d'évaluer le processus de mise en place de la REDD+ ainsi que les résultats de l'adoption de mesures incitatives de REDD+. Cette annexe propose une description succincte de chacun des instruments d'enquête et aiguille les utilisateurs de ces documents vers les sections correspondantes des recommandations techniques.

Les recommandations techniques constituent un document évolutif qui sera mis à jour au fur et à mesure de la progression de l'étude GCS-REDD+ du CIFOR. Par conséquent, nous renvoyons les lecteurs à des sections particulières plutôt qu'à des numéros de page (cf. Tableau C.1). Nous étudions chacun des instruments d'enquête par ordre décroissant de niveau d'étude : nous commençons ainsi par l'échelon du projet et terminons par celui du ménage. Les recommandations techniques contiennent également une grande quantité d'informations de première importance pour l'étude de la REDD+, dont :
- des informations générales sur le CIFOR, la REDD+ et l'étude GCS-REDD+ (Section 2 des recommandations techniques);

- les points essentiels de la méthodologie de recherche de l'étude GCS-REDD+ comprenant la problématique de la recherche (Section 3.1), le cadre théorique de référence se rapportant à l'efficacité, l'efficience, l'équité et aux cobénéfices (3E+) (Section 3.2), le but de la recherche (Section 3.3), les questions précises sur la recherche (Section 3.4), une définition opérationnelle de la REDD+ (Section 3.5), une présentation du plan expérimental " avant-après/contrôle-intervention » (BACI) (Section 3.6), l'évaluation de la mise en œuvre et de l'impact (Section 3.7), une synthèse des dimensions intensives et extensives de la recherche (Section 3.8), des éléments relatifs à la mesure et à la surveillance des émissions de carbone (Section 3.9), et enfin, une explication des choix de pays pour l'étude GCS-REDD+ (Section 3.10);

- des conseils pour mener à bien la recherche sur le terrain, avec notamment des recommandations afin de conserver son indépendance par rapport aux porteurs de projet (Section 5.4), assurer l'anonymat des personnes interrogées et la confidentialité (Section 5.5), et les principes d'excellence relatifs à la recherche de terrain (Section 5.7);

- les aspects organisationnels de l'étude GCSREDD+, dont un organigramme (Section 3.14) et un calendrier de mise de ouvre de celle-ci (Section 3.17); et

- dispositions pour l'obtention de résultats axés sur les impacts et la diffusion de ces résultats (Section 3.15 et 3.16). 
Tableau C.1. Présentation des instruments de recherche et des recommandations techniques de l'étude GCS-REDD+

\section{Instrument d'enquête}

Évaluation des porteurs de projet

\section{Description}

L'évaluation des porteurs de projet a pour objectif de :

- mener une analyse initiale de la situation dans le but d'organiser les étapes restantes de la recherche sur un site de projet donné ;

- dresser la liste de tous les acteurs qui devraient être interviewés ;

- déterminer les parties du projet qui sont respectivement en phase de conception, en cours ou terminées ;

- recueillir les informations élémentaires relatives au projet et au site du projet qui ne peuvent être obtenues de source secondaire ou par téléphone ;

- recueillir des informations sur les villages de manière à être en mesure de sélectionner un échantillon de villages sur lesquels portera la recherche du CIFOR.

Étude de la mise en œuvre de projet (SPI)

La SPI est utilisée pour :

- caractériser et enregistrer tous les éléments relatifs à la mise en œuvre de projet ;
- dresser la liste des acteurs:

- tous les acteurs principaux qui supportent les coûts de mise en œuvre et d'opportunité dans l'ensemble du projet et dans les villages étudiés, - le(s) groupe(s) d'acteurs dont on prévoit qu'ils supporteront la part des coûts d'opportunité la plus importante, à savoir : groupes dont on prévoit qu'ils renonceront à l'utilisation des sols dans la zone du projet qui leur aurait rapporté les bénéfices totaux les plus importants dans les conditions contrefactuelles ;

- quantifier les coûts totaux de mise en œuvre jusqu'au jour présent ; (Sur les sites extensifs, l'étude GCS-REDD+ s'appuiera sur le budget officiel total de la mise en œuvre ; sur les sites intensifs, elle s'attachera à connaître les apports en nature conséquents non inclus dans ce budget officiel) ;

- décomposer les coûts de mise en œuvre :

- estimer les parts en pourcentage des coûts de mise en œuvre jusqu'au jour présent consacrés (i) au FPIC et (ii) à la MNV,

estimer la part en pourcentage des coûts de mise en œuvre jusqu'au jour présent consacrée aux villages étudiés.

(Pour les sites intensifs seulement, l'étude GCSREDD+ estimera les bénéfices actuels liés aux utilisations des sols dans les villages de l'étude, gagnés par les acteurs qui (1) ne résident pas dans le village (et qui n'ont donc pas participé à I'enquête auprès des ménages ou des villages) et qui (2) devront probablement changer radicalement leur mode d'utilisation des sols en raison du projet) ;

- analyser les idées que se font différents porteurs de projet et acteurs sur la REDD+.

\section{Sections correspondantes des} recommandations techniques

3.11 Choix des projets

4.8 Évaluation des porteurs de projet

5.2 Déclaration commune de coopération

5.9 Notice explicative pour le remplissage des formulaires d'enquête

5.10 Utilisation des codes

5.11 Notice explicative pour la conduite de recherches sur les régimes de propriété des terres

8.2 Catégories d'utilisation des sols de forêts

8.3 Catégories d'utilisation des sols agricoles

8.4 Autres catégories d'utilisation des sols

Annexe 5 Instructions relatives au formulaire d'évaluation des porteurs de projet

4.17 Étude de la mise en œuvre de projet 5.11 Notice explicative pour la conduite de recherches sur les régimes de propriété des terres 


\begin{tabular}{|c|c|}
\hline $\begin{array}{l}\text { Instrument } \\
\text { d'enquête }\end{array}$ & Description \\
\hline $\begin{array}{l}\text { Questionnaire } \\
\text { destiné aux } \\
\text { grandes } \\
\text { exploitations }\end{array}$ & $\begin{array}{l}\text { Le questionnaire destiné aux grandes exploitations } \\
\text { est utilisé sur les sites intensifs à chaque fois que } \\
\text { les coûts d'opportunité les plus élevés du fait de } \\
\text { la REDD+ seront probablement supportés par de } \\
\text { grandes exploitations. Ce groupe est celui qui aura } \\
\text { probablement à renoncer au volume le plus important } \\
\text { de bénéfices liés aux utilisations des sols actuelles } \\
\text { (scénario du « laisser-faire»), du fait des changements } \\
\text { provoqués par la REDD+. Dans de nombreux sites, } \\
\text { ce " groupe d'acteurs » comprend un nombre limité } \\
\text { d'entités qui gèrent des superficies très étendues } \\
\text { dont l'exploitation est très lucrative (1-2 concessions } \\
\text { forestières ou de plantations de palmiers à huile par } \\
\text { ex.). En revanche, dans de nombreux sites au Brésil, } \\
\text { ce groupe comprend un nombre non négligeable } \\
\text { d'entreprises agricoles et d'élevage de grande taille. }\end{array}$ \\
\hline
\end{tabular}

Questionnaire destiné aux villages

\section{Sections correspondantes des} recommandations techniques

4.13 Questionnaire destiné aux grandes exploitations

5.9 Notice explicative pour le remplissage des formulaires d'enquête

5.10 Utilisation des codes

5.11 Notice explicative pour la conduite de recherches sur les régimes de propriété des terres

8.2 Catégories d'utilisation des sols de forêts

8.3 Catégories d'utilisation des sols agricoles

8.4 Autres catégories d'utilisation des sols

\subsection{Choix des villages}

4.14 Repérage cartographique des villages

4.15 Questionnaire destiné aux villages

5.7 Méthode de remémoration sur une période antérieure d'un ou deux ans

5.8 Notice explicative pour l'inscription des réponses lors d'un entretien de groupe 5.9 Notice explicative pour le remplissage des formulaires d'enquête

5.10 Utilisation des codes

5.11 Notice explicative pour la conduite de recherches sur les régimes de propriété des terres

8.2 Catégories d'utilisation des terres boisées

8.3 Catégories d'utilisation des terres agricoles 8.4 Autres catégories d'utilisation des sols
Les Sections 6-10 doivent être renseignées à partir d'informations recueillies à l'occasion d'une réunion de village. Ces sections traitent des points suivants :

1. informations de base relatives aux moyens d'existence des villageois et à leur évolution ;

2. variation de la surface, qualité et utilisation de la forêt ;

3. opinions exprimées à propos de la sécurité des droits fonciers portant sur les ressources agricoles et forestières ;

4. impressions ressenties à propos de l'évolution du bien-être ;

5. état des connaissances relatives à la REDD+ dans le village et implication des villageois dans les interventions. 


\begin{tabular}{|c|c|}
\hline Instrument & Description \\
\hline & $\begin{array}{l}\text { S'il n'existe aucune information spatiale relative aux } \\
\text { villages étudiés, ou si les limites du village ne sont pas } \\
\text { définies, il faut procéder à un repérage cartographique } \\
\text { rapide pour délimiter sommairement les villages. Ces } \\
\text { informations sont utilisées pour faire un recoupement } \\
\text { entre les données d'enquête et les résultats d'analyses } \\
\text { de l'évolution des utilisations des sols dans les villages } \\
\text { étudiés obtenus par imagerie satellitaire. Lorsqu'il } \\
\text { existe des fichiers de formes pour les villages étudiés, } \\
\text { il est inutile de faire le repérage cartographique du } \\
\text { village : il suffit de compiler les données spatiales et } \\
\text { de les transmettre au personnel du siège du CIFOR, } \\
\text { accompagnées de la base de données. }\end{array}$ \\
\hline
\end{tabular}

Évaluation des villages

Questionnaire destiné aux femmes

\section{Sections correspondantes des} recommandations techniques
3.12 Choix des villages

4.9 Evaluation des villages

5.9 Notice explicative pour le remplissage des formulaires d'enquête

5.10 Utilisation des codes

8.1 Définition formelle d'un ménage

Annexe 1 Instructions relatives au formulaire d'évaluation des villages

Annexe 2 Formulaire d'évaluation des villages leur similitude sera grande, plus nous pourrons être certains que les différences constatées entre les villages d'intervention et de contrôle pendant les phases de l' « avant » et de l' « après » projet sont imputables à la REDD+ et non à une autre cause. Le formulaire d'évaluation des villages doit être employé parallèlement à l'exercice 27 du formulaire d'évaluation des porteurs de projet.

Le questionnaire des femmes a trois objectifs. Premièrement, il permet aux femmes de se faire entendre dans le cadre de l'étude. Deuxièmement, il permet d'obtenir des données liées à l'expérience et aux connaissances particulières des femmes. Troisièmement, il fournit des informations permettant de comparer les activités de subsistance et la vision des femmes avec celles des hommes.

Le questionnaire destiné aux femmes se compose de quatre parties :

1. moyens d'existence des femmes dans le village et leur évolution ;

2. participation des femmes aux décisions du village ;

3. impressions des femmes quant à l'évolution de leur bien-être ;

4. connaissances des femmes au sujet de la REDD+ et leur implication dans la REDD+.
4.16 Questionnaire destiné aux femmes 5.7 Méthode de remémoration sur une période antérieure d'un ou deux ans 5.8 Notice explicative pour l'inscription des réponses lors d'un entretien de groupe 5.9 Notice explicative pour le remplissage des formulaires d'enquête

5.10 Utilisation des codes

5.11 Notice explicative pour la conduite de recherches sur les régimes de propriété des terres

8.2 Catégories d'utilisation des terres boisées

8.3 Catégories d'utilisation des terres agricoles

8.4 Autres catégories d'utilisation des sols 


\begin{tabular}{|c|c|c|}
\hline $\begin{array}{l}\text { Instrument } \\
\text { d'enquête }\end{array}$ & Description & $\begin{array}{l}\text { Sections correspondantes des } \\
\text { recommandations techniques }\end{array}$ \\
\hline $\begin{array}{l}\text { Questionnaire } \\
\text { destiné aux } \\
\text { ménages }\end{array}$ & $\begin{array}{l}\text { Le questionnaire destiné aux ménages constitue le } \\
\text { seul instrument de l'étude GCS-REDD+ permettant } \\
\text { d'obtenir des données au niveau du ménage. C'est } \\
\text { le moyen principal dont nous disposons pour nous } \\
\text { informer en détail sur les sites intensifs ; c'est aussi } \\
\text { notre point d'entrée principal pour la collecte de } \\
\text { données socioéconomiques dans l'approche BACl. } \\
\text { Le questionnaire destiné aux ménages a } \\
\text { principalement pour fonction de : } \\
\text { - mesurer l'effet potentiel de la REDD+ sur le bien- } \\
\text { être du ménage, par des mesures objectives } \\
\text { (moyens d'existence, biens, revenu sur 12 mois) et } \\
\text { subjectives (niveau de bien-être ressenti, causes des } \\
\text { changements pour ceux qui en connaissent); } \\
\text { - mesurer l'effet potentiel de la REDD+ sur } \\
\text { l'utilisation des sols et des ressources à l'échelon du } \\
\text { ménage ; } \\
\text { renseigner sur les connaissances des ménages } \\
\text { au sujet de la REDD+ et leur implication dans les } \\
\text { activités de la REDD+. } \\
\text { La mise en œuvre réussie du questionnaire destiné aux } \\
\text { ménages dépend de la connaissance détaillée et de la } \\
\text { parfaite maîtrise de la méthode de remémoration sur } \\
\text { une période antérieure d'un ou de deux ans. }\end{array}$ & $\begin{array}{l}\text { 4.11 Obtention ou création d'une liste des } \\
\text { ménages } \\
\text { 4.12 Questionnaire destiné aux ménages } \\
\text { 5.7 Méthode de remémoration sur une période } \\
\text { antérieure d'un ou deux ans } \\
\text { 5.9 Notice explicative pour le remplissage des } \\
\text { formulaires d'enquête } \\
\text { 5.10 Utilisation des codes } \\
5.11 \text { Notice explicative pour la conduite de } \\
\text { recherches sur les régimes de propriété des } \\
\text { terres } \\
\text { 7.1 Notice explicative pour la constitution d'un } \\
\text { échantillon aléatoire de ménages } \\
\text { 8.1 Définition formelle d'un ménage } \\
\text { 8.2 Catégories d'utilisation des terres boisées } \\
\text { 8.3 Catégories d'utilisation des terres agricoles } \\
\text { 8.4 Autres catégories d'utilisation des sols }\end{array}$ \\
\hline $\begin{array}{l}\text { Formulaire de } \\
\text { l'échelle des } \\
\text { matériaux de } \\
\text { construction des } \\
\text { habitations }\end{array}$ & $\begin{array}{l}\text { L'échelle des matériaux de construction des } \\
\text { habitations est un formulaire qui est utilisé pour } \\
\text { définir une échelle de valeur propre au village } \\
\text { (basse, moyenne, élevée) relative aux matériaux } \\
\text { de construction des habitations de ce village. Ce } \\
\text { formulaire s'emploie dans le but spécifique de } \\
\text { déterminer les codes à utiliser pour remplir le Tableau } \\
2 C \text { dans le questionnaire d'enquête destiné aux } \\
\text { ménages. Les informations de ce tableau font partie } \\
\text { des indicateurs du bien-être relatif des ménages du } \\
\text { village. }\end{array}$ & $\begin{array}{l}\text { 4.10 Echelle des matériaux de construction des } \\
\text { habitations } \\
\text { Annexe } 6 \text { Formulaire de l'échelle des matériaux } \\
\text { de construction des habitations }\end{array}$ \\
\hline
\end{tabular}




Les Documents occasionnels du CIFOR contiennent des résultats de recherche qui sont importants pour la foresterie tropicale. Le contenu est revu par des pairs en interne comme en externe.

L'Étude comparative mondiale du CIFOR sur la REDD+ qui s'étale sur plusieurs années a pour objectif d'informer les responsables politiques, les praticiens et les donateurs sur les approches efficaces en matière de réduction des émissions dues à la déforestation et à la dégradation des forêts et de renforcement des stocks de carbone forestier dans les pays en développement. Nous remercions vivement les organisations suivantes pour leur appui : I'Agence norvégienne de coopération pour le développement, l'Agence australienne d'aide au développement international, le ministère britannique du développement international, la Commission européenne, le Département finlandais pour la coopération au développement international, la Fondation David et Lucile Packard, le Programme sur les forêts, l'Agence américaine pour le développement international et le Service des forêts du Département de l'agriculture aux États-Unis. Development
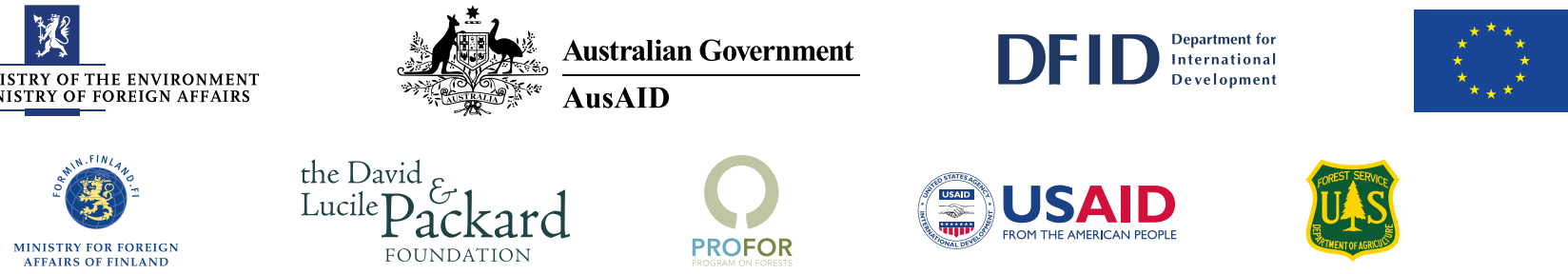

$$
\begin{aligned}
& \text { the David } \\
& \text { Lucile Packard } \\
& \text { Foundation }
\end{aligned}
$$
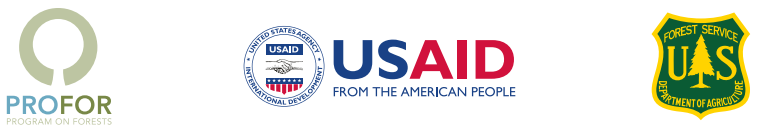

\section{Centre de recherche forestière internationale}

Le CIFOR défend le bien-être humain, la conservation de l'environnement et l'équité par ses activités de recherche qui visent à éclairer les politiques et les pratiques affectant les forêts dans les pays en développement. Le CIFOR est I'un des 15 centres au sein du Groupe consultatif sur la recherche agricole internationale (CGIAR). Le siège du CIFOR est situé à Bogor, en Indonésie. Le CIFOR a également des bureaux en Asie, en Afrique et en Amérique du Sud. 